\author{
Supporting Information \\ for
}

\title{
Annulation of diaryl(aryl)phosphenes and cyclic imines to access benzo- $\delta$-phospholactams
}

\author{
Yun Luo, and Jiaxi $\mathrm{Xu}^{*}$ \\ State Key Laboratory of Chemical Resource Engineering, Department of Organic Chemistry, College \\ of Chemistry, Beijing University of Chemical Technology, Beijing 100029, People's Republic of \\ China. E-mail: jxxu@mail.buct.edu.cn
}




\section{Contents}

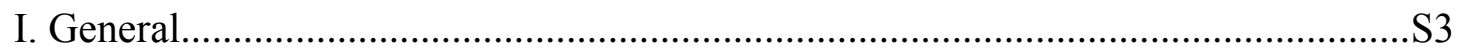

II. General Procedure for the Synthesis of Diazo Compounds 1 ................................S4

III. General Procedure for the Synthesis of Cyclic Imines 2 ….................................S9

IV.General Procedure for the Synthesis of Products 3 ............................................S15

V. Procedure for the Large-Scaled Preparation of Product 3b.................................S34

VI. Procedure for the Suzuki Coupling Reaction of Compound 3g .........................S35

VII. Crystal Data and Structure Refinement for Compound 3c....................S36

VIII. Competitive Experiments and Copies of NMR Spectra ..................................S38

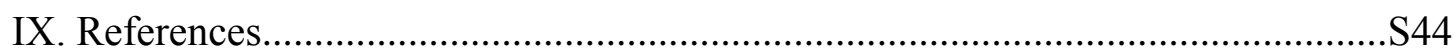

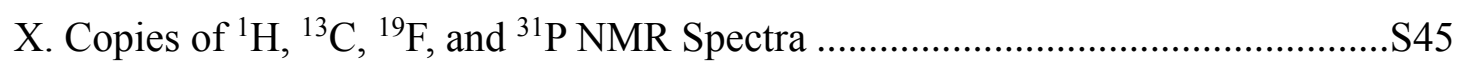




\section{General}

Unless otherwise noted, all materials were purchased from commercial suppliers. MeCN, DCE, THF, $\mathrm{PhCl}$ were refluxed over $\mathrm{CaH}_{2}$, and freshly distilled prior to use. Microwave reactions were performed using a commercial microwave reactor with an external IR sensor and in a closed reaction vessel. Flash column chromatography was performed using silica gel (normal phase, 200-300 mesh) from Branch of Qingdao Haiyang Chemicals. Petroleum ether used for column chromatography were $60-90{ }^{\circ} \mathrm{C}$ fraction, and the removal of residue solvent was accomplished under rotovap with repeated azeotrope with chloroform, and then evaporation under vacuum $(<1 \mathrm{mmHg}$ pressure). Reactions were monitored by thin-layer chromatography on silica gel 60F254 coated $0.2 \mathrm{~mm}$ plates from Institute of Yantai Chemical Industry. The plates were visualized under UV light, as well as other TLC stains (potassium permanganate: 1\% potassium permanganate in water; iodine: $10 \mathrm{~g}$ of iodine absorbed on $30 \mathrm{~g}$ silica gel). ${ }^{1} \mathrm{H}(400 \mathrm{MHz}),{ }^{13} \mathrm{C}(101 \mathrm{MHz}),{ }^{31} \mathrm{P}(162 \mathrm{MHz})$, and ${ }^{19} \mathrm{~F}$ NMR (376 MHz) spectra were recorded on a 400 NMR spectrometer usually with TMS as an internal standard in $\mathrm{CDCl}_{3}$ solution and the chemical shifts $(\delta)$ were reported in parts per million (ppm). Melting points were obtained on a melting point apparatus and are uncorrected. The IR spectra $\left(\mathrm{KBr}\right.$ pellets, $\left.v\left[\mathrm{~cm}^{-1}\right]\right)$ were taken on a FTIR spectrometer. HRMS measurements were carried out on an LC/MSD TOF mass spectrometer. The thin layer chromatography silica gel preparative plates were purchased from Anhui Liangchen Silicon Material Co. Ltd. 


\section{General Procedure for the Synthesis of Diazo Compounds 1}

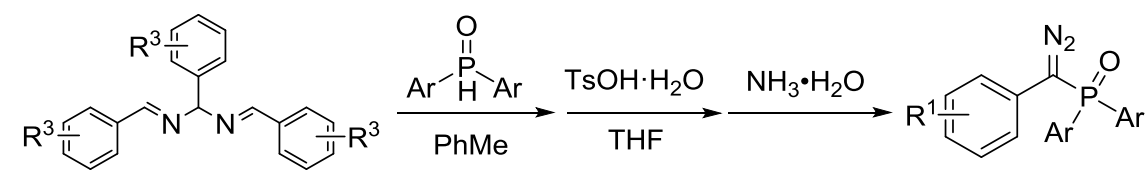

The diazo compounds 1 were synthesized according to the reported procedures. ${ }^{1,2}$ Benzaldazine derivatives $^{2}(5 \mathrm{mmol})$ and diarylphosphine oxides $(10 \mathrm{mmol})$ were dissolved in $40 \mathrm{~mL}$ of toluene. The solution was stirred at room temperature overnight, and during the time a large amount of white solid was formed. The resulting solid was filtered off and washed with petroleum ether for several times. Then the obtained solid was dissolved in $40 \mathrm{~mL}$ of tetrahydrofuran. After addition of $p$-toluenesulfonic acid hydrate (12 mmol, $2.28 \mathrm{~g}$ ), the mixture was stirred at room temperature for $5 \mathrm{~h}$. The resulting solid was filtered off and washed with tetrahydrofuran for several times. The obtained solid was dissolved in $30 \mathrm{~mL}$ of $15 \%$ aqueous ammonia, and the mixture was stirred at room temperature for $1 \mathrm{~h}$. After finishing stirring, the mixture was extracted with chloroform $(3 \times 20 \mathrm{~mL})$. The combined organic phase was dried over anhydrous sodium sulfate. After removal partial solvent, isoamyl nitrite (12 mmol, $1.44 \mathrm{~g})$ and glacial acetic acid ( $5 \mathrm{mmol}, 0.3 \mathrm{~mL}$ ) were added. The mixture was refluxed for $1 \mathrm{~h}$. After finishing the reaction, the mixture was washed successively with saturated $\mathrm{NaHCO}_{3}$ solution and brine, dried over anhydrous sodium sulfate, and concentrated under the reduce pressure. The desire product was purified by column chromatography on silica gel (PE:EA = 5:1 to 3:1, v/v). In some cases, compounds 1 were synthesized in small scales due to the lack of diarylphosphine oxides.

\section{(Diazo(phenyl)methyl)diphenylphosphine oxide (1a)}<smiles>N#CC(=O)P(=O)(c1ccccc1)c1ccccc1</smiles>

Orange crystals (1.02 g, 32\%); mp. $162-164{ }^{\circ} \mathrm{C}$ (Lit ${ }^{1}: \mathrm{mp} .155-156$ $\left.{ }^{\circ} \mathrm{C}\right)$. IR (KBr) $v\left(\mathrm{~cm}^{-1}\right) 3055,2923,2073,1594,1493,1438,1196,1117,1099,951 .{ }^{1} \mathrm{H}$ NMR (400 MHz, $\left.\mathrm{CDCl}_{3}\right) \delta 7.82(\mathrm{~m}, 4 \mathrm{H}), 7.60-7.54(\mathrm{~m}, 2 \mathrm{H}), 7.53-7.46(\mathrm{~m}, 4 \mathrm{H})$, $7.29-7.21(\mathrm{~m}, 4 \mathrm{H}), 7.11-7.06(\mathrm{~m}, 1 \mathrm{H}) .{ }^{13} \mathrm{C} \mathrm{NMR}\left(101 \mathrm{MHz}, \mathrm{CDCl}_{3}\right) \delta 132.6(\mathrm{~d}, J=$ $2.4 \mathrm{~Hz}), 131.8(\mathrm{~d}, J=10.2 \mathrm{~Hz}), 130.8(\mathrm{~d}, J=113.2 \mathrm{~Hz}), 129.2,128.9(\mathrm{~d}, J=12.9 \mathrm{~Hz})$, 
$127.2(\mathrm{~d}, J=7.6 \mathrm{~Hz}), 125.3,123.8(\mathrm{~d}, J=3.5 \mathrm{~Hz}), 52.8(\mathrm{~d}, J=119.1 \mathrm{~Hz}) .{ }^{31} \mathrm{P}$ NMR $\left(162 \mathrm{MHz}, \mathrm{CDCl}_{3}\right) \delta$ 26.6. HRMS (ESI) calcd for $\mathrm{C}_{19} \mathrm{H}_{16} \mathrm{~N}_{2} \mathrm{OP}^{+}[\mathrm{M}+\mathrm{H}]^{+} m / z: 319.0995$; found 319.0990 .

(Diazo(2-fluorophenyl)methyl)diphenylphosphine oxide (1b)<smiles></smiles>
Yellow crystals $(1.38 \mathrm{~g}, 58 \%)$; mp. $130-132{ }^{\circ} \mathrm{C}$. IR $(\mathrm{KBr}) v\left(\mathrm{~cm}^{-1}\right)$ 3060, 2923, 2061, 1725, 1599, 1500, 1289, 1199, 1116, 808, 751, 661, 551. ${ }^{1} \mathrm{H}$ NMR $\left(400 \mathrm{MHz}^{\mathrm{CDCl}} \mathrm{CD}_{3} \delta 7.76(\mathrm{dd}, J=12.2,7.6 \mathrm{~Hz}, 4 \mathrm{H}), 7.50-7.33(\mathrm{~m}, 7 \mathrm{H}), 7.07-6.86\right.$ $(\mathrm{m}, 3 \mathrm{H}) .{ }^{13} \mathrm{C}$ NMR $\left(101 \mathrm{MHz}, \mathrm{CDCl}_{3}\right) \delta 159.3\left(\mathrm{dd}, J_{C-F, C-P}=246.6,5.1 \mathrm{~Hz}\right), 132.6(\mathrm{~d}$, $J=2.9 \mathrm{~Hz}), 131.8(\mathrm{~d}, J=10.1 \mathrm{~Hz}), 130.4\left(\mathrm{~d}, J_{C-P}=113.5 \mathrm{~Hz}\right), 128.9(\mathrm{~d}, J=12.9 \mathrm{~Hz})$, $128.3\left(\mathrm{dd}, J_{C-F, C-P}=2.1,2.1 \mathrm{~Hz}\right), 128.0(\mathrm{~d}, J=8.1 \mathrm{~Hz}), 124.8(\mathrm{~d}, J=3.5 \mathrm{~Hz}), 115.8(\mathrm{~d}$, $J=21.0 \mathrm{~Hz}) .{ }^{31} \mathrm{P}$ NMR $\left(162 \mathrm{MHz}, \mathrm{CDCl}_{3}\right) \delta 27.9 .{ }^{19} \mathrm{~F} \mathrm{NMR}\left(376 \mathrm{MHz}, \mathrm{CDCl}_{3}\right) \delta-$ 114.5. HRMS (ESI) calcd for $\mathrm{C}_{19} \mathrm{H}_{15} \mathrm{FN}_{2} \mathrm{OP}^{+}[\mathrm{M}+\mathrm{H}]^{+} \mathrm{m} / z$ : 337.0901 ; found 337.0902 .

\section{(Diazo(4-methylphenyl)methyl)diphenylphosphine oxide (1c)}<smiles>Cc1ccc(C(=N)P(=O)(c2ccccc2)c2ccccc2)cc1</smiles>

Yellow crystals ( $630 \mathrm{mg}, 32 \%)$; mp. $146-148^{\circ} \mathrm{C}$. IR (KBr) $v\left(\mathrm{~cm}^{-}\right.$ 1) $3060,2973,2927,2071,1619,1509,1437,1325,1197,1049,723,702 .{ }^{1} \mathrm{H}$ NMR $\left(400 \mathrm{MHz}, \mathrm{CDCl}_{3}\right) \delta 7.88-7.77(\mathrm{~m}, 4 \mathrm{H}), 7.57-7.44(\mathrm{~m}, 6 \mathrm{H}), 7.15-7.03(\mathrm{~m}, 4 \mathrm{H})$, $2.26(\mathrm{~s}, 3 \mathrm{H}) .{ }^{13} \mathrm{C} \mathrm{NMR}\left(101 \mathrm{MHz}, \mathrm{CDCl}_{3}\right) \delta 135.2,132.5(\mathrm{~d}, J=2.2 \mathrm{~Hz}), 131.8(\mathrm{~d}, J=$ $10.1 \mathrm{~Hz}), 130.8$ (d, $J=113.2 \mathrm{~Hz}), 129.9,128.9$ (d, $J=12.9 \mathrm{~Hz}), 123.8$ (d, $J=3.3 \mathrm{~Hz})$, $123.6(\mathrm{~d}, J=7.8 \mathrm{~Hz}), 52.4(\mathrm{~d}, J=120.5 \mathrm{~Hz}), 20.9 .{ }^{31} \mathrm{P} \mathrm{NMR}\left(162 \mathrm{MHz}, \mathrm{CDCl}_{3}\right) \delta 26.8$. HRMS (ESI) calcd for $\mathrm{C}_{20} \mathrm{H}_{18} \mathrm{~N}_{2} \mathrm{OP}^{+}[\mathrm{M}+\mathrm{H}]^{+} m / z$ : 333.1151 ; found 333.1153 .

\section{((4-Chlorophenyl)(diazo)methyl)diphenylphosphine oxide (1d)}


<smiles>N=C(c1ccc(Cl)cc1)P(=O)(c1ccccc1)c1ccccc1</smiles>

Yellow crystals $(0.294 \mathrm{~g}, 14 \%)$; mp. $152-154{ }^{\circ} \mathrm{C}$. IR $(\mathrm{KBr}) v$ $\left(\mathrm{cm}^{-1}\right)$ 3060, 2971, 2926, 2065, 1510, 1459, 1438, 1322, 1208, 1089, 1048, 880, 732, 696. ${ }^{1} \mathrm{H}$ NMR $\left(400 \mathrm{MHz}, \mathrm{CDCl}_{3}\right) \delta 7.85-7.76(\mathrm{~m}, 4 \mathrm{H}), 7.62-7.47$ (m, 6H), $7.27-$ $7.12(\mathrm{~m}, 4 \mathrm{H}) .{ }^{13} \mathrm{C}$ NMR $\left(101 \mathrm{MHz}, \mathrm{CDCl}_{3}\right) \delta 132.8(\mathrm{~d}, J=2.2 \mathrm{~Hz}), 131.8(\mathrm{~d}, J=10.2$ $\mathrm{Hz}), 130.4(\mathrm{~d}, J=113.5 \mathrm{~Hz}), 129.4,129.0(\mathrm{~d}, J=12.9 \mathrm{~Hz}), 128.8(\mathrm{~d}, J=12.3 \mathrm{~Hz})$, $126.0(\mathrm{~d}, J=8.3 \mathrm{~Hz}), 124.9(\mathrm{~d}, J=3.3 \mathrm{~Hz}) .{ }^{31} \mathrm{P} \mathrm{NMR}\left(162 \mathrm{MHz}, \mathrm{CDCl}_{3}\right) \delta 26.6$. HRMS (ESI) calcd for $\mathrm{C}_{19} \mathrm{H}_{15} \mathrm{ClN}_{2} \mathrm{OP}^{+}[\mathrm{M}+\mathrm{H}]^{+} m / z$ : 353.0605; found 353.0597.

\section{(Diazo(4-(trifluoromethyl)phenyl)methyl)diphenylphosphine oxide (1e)}<smiles>[Y]c1ccc(P(=O)(C(=N)c2ccc(C(F)(F)F)cc2)c2ccccc2)cc1</smiles>

Yellow crystals $(173 \mathrm{mg}, 9 \%)$; mp. $165-166{ }^{\circ} \mathrm{C} . \mathrm{IR}(\mathrm{KBr}) v$ $\left(\mathrm{cm}^{-1}\right)$ 3361, 2922, 2095, 1613, 1439, 1327, 1192, 1071, 566. ${ }^{1} \mathrm{H}$ NMR (400 MHz, $\left.\mathrm{CDCl}_{3}\right) \delta 7.85-7.79(\mathrm{~m}, 4 \mathrm{H}), 7.62-7.58(\mathrm{~m}, 2 \mathrm{H}), 7.54-7.49(\mathrm{~m}, 6 \mathrm{H}), 7.32(\mathrm{~d}, J=$ $8.4 \mathrm{~Hz}, 2 \mathrm{H}) .{ }^{13} \mathrm{C} \mathrm{NMR}\left(101 \mathrm{MHz}, \mathrm{CDCl}_{3}\right) \delta 133.1(\mathrm{~d}, J=2.9 \mathrm{~Hz}), 132.0(\mathrm{~d}, J=6.4 \mathrm{~Hz})$, $131.9(\mathrm{~d}, J=4.8 \mathrm{~Hz}), 131.0,130.1\left(\mathrm{q}, J_{C-F}=270.2 \mathrm{~Hz}\right), 129.8,129.3(\mathrm{~d}, J=5.0 \mathrm{~Hz})$, $129.2(\mathrm{~d}, J=5.3 \mathrm{~Hz}), 126.2\left(\mathrm{q}, J_{C-F}=4.0 \mathrm{~Hz}\right), 123.5(\mathrm{~d}, J=3.7 \mathrm{~Hz}) \cdot{ }^{19} \mathrm{~F}$ NMR $(376$ $\left.\mathrm{MHz}, \mathrm{CDCl}_{3}\right) \delta-62.46 .{ }^{31} \mathrm{P} \mathrm{NMR}\left(162 \mathrm{MHz}, \mathrm{CDCl}_{3}\right) \delta$ 26.3. HRMS (ESI) calcd for $\mathrm{C}_{20} \mathrm{H}_{15} \mathrm{~F}_{3} \mathrm{~N}_{2} \mathrm{OP}^{+}[\mathrm{M}+\mathrm{H}]^{+} m / z$ : 387.0869; found 387.0872 .

\section{(Diazo(4-methoxyphenyl)methyl)diphenylphosphine oxide (1g)}<smiles>COc1ccc(C(=N)P(=O)(c2ccccc2)c2ccccc2)cc1</smiles>

Yellow crystals (35 mg, $2 \%$ ); mp. $145-146^{\circ} \mathrm{C}$. IR (KBr) $v$ $\left(\mathrm{cm}^{-1}\right) 3438,2928,2061,1648,1608,1511,1438,1252,1196,1118,827,698 .{ }^{1} \mathrm{H}$ NMR $\left(400 \mathrm{MHz}, \mathrm{CDCl}_{3}\right) \delta 7.84-7.77(\mathrm{~m}, 4 \mathrm{H}), 7.59-7.52(\mathrm{~m}, 2 \mathrm{H}), 7.50-7.46(\mathrm{~m}, 4 \mathrm{H})$, $7.19-7.16(\mathrm{~m} \mathrm{2H}), 6.86-6.80(\mathrm{~m}, 2 \mathrm{H}), 3.74(\mathrm{~s}, 3 \mathrm{H}) .{ }^{13} \mathrm{C} \mathrm{NMR}\left(101 \mathrm{MHz}, \mathrm{CDCl}_{3}\right) \delta$ 
$157.9,132.7(\mathrm{~d}, J=2.9 \mathrm{~Hz}), 132.0(\mathrm{~d}, J=10.1 \mathrm{~Hz}), 131.6,130.5,129.0(\mathrm{~d}, J=12.9$ $\mathrm{Hz}), 125.8(\mathrm{~d}, J=3.6 \mathrm{~Hz}), 118.5(\mathrm{~d}, J=7.9 \mathrm{~Hz}), 115.1,55.4,51.8(\mathrm{~d}, J=121.4 \mathrm{~Hz})$. ${ }^{31} \mathrm{P}$ NMR (162 MHz, $\left.\mathrm{CDCl}_{3}\right) \delta$ 26.9. HRMS (ESI) calcd for $\mathrm{C}_{20} \mathrm{H}_{18} \mathrm{~N}_{2} \mathrm{O}_{2} \mathrm{P}^{+}[\mathrm{M}+\mathrm{H}]^{+} m / z$ : 349.1100; found 349.1092.

\section{(Diazo(phenyl)methyl)di(4-methylphenyl)phosphine oxide (1g)}

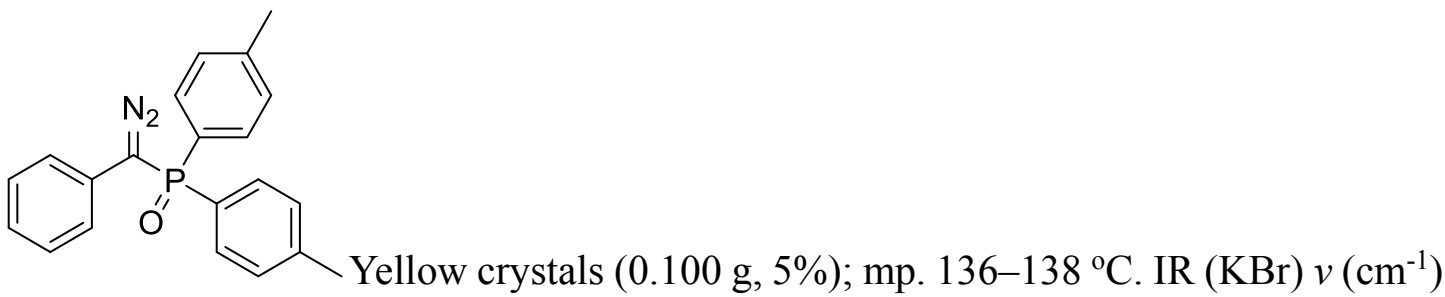
2973, 2928, 2073, 1619, 1438, 1324, 1090, 1049, 881, 748, 699, 563. ${ }^{1} \mathrm{H}$ NMR (400 $\left.\mathrm{MHz}, \mathrm{CDCl}_{3}\right) \delta 7.69(\mathrm{~m}, 4 \mathrm{H}), 7.31-7.20(\mathrm{~m}, 8 \mathrm{H}), 7.11-7.04(\mathrm{~m}, 1 \mathrm{H}), 2.39(\mathrm{~s}, 6 \mathrm{H})$. ${ }^{13} \mathrm{C}$ NMR (101 MHz, $\left.\mathrm{CDCl}_{3}\right) \delta 143.2(\mathrm{~d}, J=2.9 \mathrm{~Hz}), 131.8(\mathrm{~d}, J=10.4 \mathrm{~Hz}), 129.7$ (d, $J=13.3 \mathrm{~Hz}), 129.1,127.7(\mathrm{~d}, J=115.8 \mathrm{~Hz}), 127.5(\mathrm{~d}, J=7.6 \mathrm{~Hz}), 125.2,123.8(\mathrm{~d}, J=$ $3.7 \mathrm{~Hz}), 53.0(\mathrm{~d}, J=118.9 \mathrm{~Hz}), 21.7 .{ }^{31} \mathrm{P} \mathrm{NMR}\left(162 \mathrm{MHz}, \mathrm{CDCl}_{3}\right) \delta 27.0$. HRMS (ESI) calcd for $\mathrm{C}_{21} \mathrm{H}_{20} \mathrm{~N}_{2} \mathrm{OP}^{+}[\mathrm{M}+\mathrm{H}]^{+} m / z$ : 347.1308; found 347.1308.

\section{Bis(4-chlorophenyl)(diazo(phenyl)methyl)phosphine oxide (1h)}<smiles>N=C(c1ccccc1)P(=O)(c1ccc(Cl)cc1)c1ccc(Cl)cc1</smiles>

$\mathrm{Cl}$ Orange crystals $(900 \mathrm{mg}, 23 \%) ; \mathrm{mp} .140-142^{\circ} \mathrm{C} . \mathrm{IR}(\mathrm{KBr}) v\left(\mathrm{~cm}^{-}\right.$ 1) $3059,2928,2064,1580,1495,1480,1389,1289,1207,1180,1117,1086,1014,950$, 821, 757, 746, 691, 567. ${ }^{1} \mathrm{H}$ NMR (400 MHz, $\left.\mathrm{CDCl}_{3}\right) \delta 7.74(\mathrm{dd}, J=12.2,8.4 \mathrm{~Hz}, 4 \mathrm{H})$, $7.48(\mathrm{dd}, J=8.4,2.4 \mathrm{~Hz}, 4 \mathrm{H}), 7.32-7.24(\mathrm{~m}, 2 \mathrm{H}), 7.20(\mathrm{~d}, J=7.6 \mathrm{~Hz}, 2 \mathrm{H}), 7.11(\mathrm{t}, J$ $=7.3 \mathrm{~Hz}, 1 \mathrm{H}) .{ }^{13} \mathrm{C} \mathrm{NMR}\left(101 \mathrm{MHz}, \mathrm{CDCl}_{3}\right) \delta 139.6(\mathrm{~d}, J=3.6 \mathrm{~Hz}), 133.1(\mathrm{~d}, J=11.1$ Hz), 129.5, 129.4 (d, $J=6.1 \mathrm{~Hz}), 128.9$ (d, $J=115.5 \mathrm{~Hz}), 126.5(\mathrm{~d}, J=7.9 \mathrm{~Hz}), 125.8$, $123.8(\mathrm{~d}, J=3.8 \mathrm{~Hz}), 52.7(\mathrm{~d}, J=123.3 \mathrm{~Hz}) .{ }^{31} \mathrm{P}$ NMR $\left(162 \mathrm{MHz}, \mathrm{CDCl}_{3}\right) \delta 24.9$. HRMS (ESI) calcd for $\mathrm{C}_{19} \mathrm{H}_{14} \mathrm{Cl}_{2} \mathrm{~N}_{2} \mathrm{OP}^{+}[\mathrm{M}+\mathrm{H}]^{+} \mathrm{m} / z$ : 387.0215; found 387.0214. 
(Diazo(pyridin-3-yl)methyl)diphenylphosphine oxide (1h)

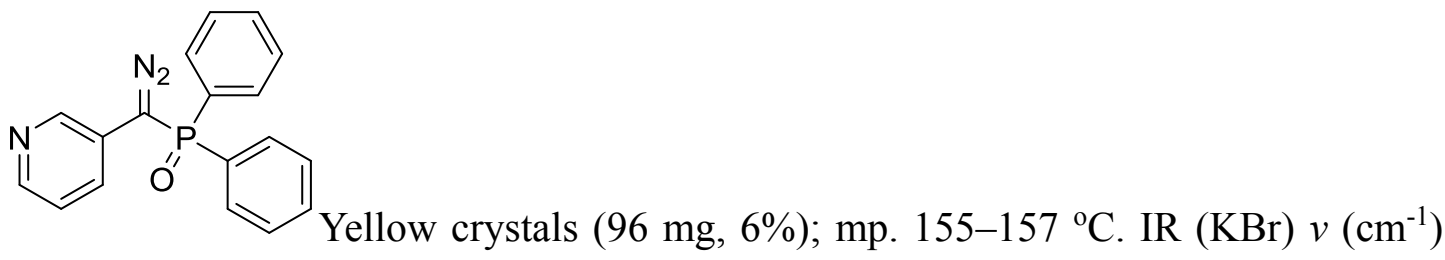
3421, 2956, 2925, 2852, 2360, 2341, 2068, 1437, 1295, 1195, 695, 561. ${ }^{1} \mathrm{H}$ NMR (400 $\left.\mathrm{MHz} \mathrm{CDCl}_{3}\right) \delta 8.48(\mathrm{~d}, J=2.4 \mathrm{~Hz}, 1 \mathrm{H}), 8.32\left(\mathrm{dd}, J=4.8 \mathrm{~Hz}, J_{P-H}=1.3 \mathrm{~Hz}, 1 \mathrm{H}\right), 7.89$ $-7.78(\mathrm{~m}, 4 \mathrm{H}), 7.66-7.55(\mathrm{~m}, 3 \mathrm{H}), 7.55-7.48(\mathrm{~m}, 4 \mathrm{H}), 7.15(\mathrm{dd}, J=8.1,4.8 \mathrm{~Hz}, 1 \mathrm{H})$. ${ }^{13} \mathrm{C} \mathrm{NMR}\left(101 \mathrm{MHz}, \mathrm{CDCl}_{3}\right) \delta 146.6,145.0(\mathrm{~d}, J=4.1 \mathrm{~Hz}), 133.1(\mathrm{~d}, J=2.9 \mathrm{~Hz}), 132.0$ $(\mathrm{d}, J=5.0 \mathrm{~Hz}), 131.9(\mathrm{~d}, J=6.3 \mathrm{~Hz}), 131.1(\mathrm{~d}, J=3.1 \mathrm{~Hz}), 130.8,129.7(\mathrm{~d}, J=2.2 \mathrm{~Hz})$, 129.3, 129.2, 126.8, 123.8. ${ }^{31} \mathrm{P}$ NMR (162 MHz, $\left.\mathrm{CDCl}_{3}\right) \delta 26.4$. HRMS (ESI) calcd for $\mathrm{C}_{18} \mathrm{H}_{15} \mathrm{~N}_{3} \mathrm{OP}^{+}[\mathrm{M}+\mathrm{H}]^{+} \mathrm{m} / z$ : 320.0947 ; found 320.0949 .

\section{Procedure for the synthesis of (diazomethyl)diphenylphosphine oxide (1j)}

A solution of (aminomethyl)diphenylphosphine oxide ${ }^{3}$ (2.31 g, $10 \mathrm{mmol}$ ), isoamyl nitrite (12 mmol, $1.44 \mathrm{~g})$ and glacial acetic acid (5 mmol, $0.3 \mathrm{~mL})$ in $\mathrm{CHCl}_{3}(30 \mathrm{~mL})$ was refluxed for $1 \mathrm{~h}$. After finishing the reaction, the mixture was washed successively with saturated $\mathrm{NaHCO}_{3}$ solution and brine, dried over anhydrous sodium sulfate, and concentrated under the reduce pressure. The residue was purified by column chromatography on silica gel $(\mathrm{PE}: \mathrm{EA}=3: 1$ to $1: 1, v / v)$ to give yellowish crystals.

\section{(Diazomethyl)diphenylphosphine oxide (1j) ${ }^{4}$}<smiles>N#CC(=O)c1ccccc1</smiles>

Yellowish crystals $(1.75 \mathrm{~g}, 72 \%)$; mp. $62-64{ }^{\circ} \mathrm{C} . \mathrm{IR}(\mathrm{KBr}) v\left(\mathrm{~cm}^{-1}\right) 3055$, 2923, 2073, 1594, 1493, 1438, 1196, 1117, 1099, 951. ${ }^{1} \mathrm{H}$ NMR (400 MHz, $\left.\mathrm{CDCl}_{3}\right) \delta$ $7.86-7.79(\mathrm{~m}, 4 \mathrm{H}), 7.58-7.47(\mathrm{~m}, 6 \mathrm{H}), 4.18(\mathrm{~d}, J=12.0 \mathrm{~Hz}, 1 \mathrm{H}) .{ }^{13} \mathrm{C}$ NMR $(101$ $\left.\mathrm{MHz}, \mathrm{CDCl}_{3}\right) \delta 132.4(\mathrm{~d}, J=2.9 \mathrm{~Hz}), 131.9(\mathrm{~d}, J=114.6 \mathrm{~Hz}), 131.4(\mathrm{~d}, J=10.2 \mathrm{~Hz})$, $128.8(\mathrm{~d}, J=12.8 \mathrm{~Hz}) .{ }^{31} \mathrm{P} \mathrm{NMR}\left(162 \mathrm{MHz}, \mathrm{CDCl}_{3}\right) \delta$ 25.2. HRMS (ESI) calcd for $\mathrm{C}_{13} \mathrm{H}_{12} \mathrm{~N}_{2} \mathrm{OP}^{+}[\mathrm{M}+\mathrm{H}]^{+} m / z: 243.0682$; found 243.0678. 
Procedure for the synthesis of ethyl (diazo(phenyl)methyl)(phenyl)phosphinate (1k)

Ethyl (diazo(phenyl)methyl)(phenyl)phosphinate (1k) was synthesized according to the reported procedures. ${ }^{5}$<smiles>N#CC(=O)P(=O)(Oc1ccccc1)c1ccccc1</smiles>

Yellow oil (700 mg, 24\%). ${ }^{1} \mathrm{H}$ NMR (400 MHz, $\left.\mathrm{CDCl}_{3}\right) \delta 7.84-7.73$ (m, 2H), $7.49-7.34(\mathrm{~m}, 3 \mathrm{H}), 7.23-7.15(\mathrm{~m}, 2 \mathrm{H}), 7.08$ (d, J=7.6 Hz, 2H), $7.03-6.96$ (m, 1H), $4.24(\mathrm{ddq}, J=14.2,10.1,7.1 \mathrm{~Hz}, 1 \mathrm{H}), 4.11(\mathrm{ddq}, J=14.2,10.1,7.2 \mathrm{~Hz}, 1 \mathrm{H})$, $1.32(\mathrm{t}, J=7.2 \mathrm{~Hz}, 3 \mathrm{H}) .{ }^{13} \mathrm{C} \mathrm{NMR}\left(101 \mathrm{MHz}, \mathrm{CDCl}_{3}\right) \delta 132.6(\mathrm{~d}, J=2.6 \mathrm{~Hz}), 131.0(\mathrm{~d}$, $J=10.7 \mathrm{~Hz}), 129.1$ (s), 128.7 (d, $J=14.5 \mathrm{~Hz}), 127.0(\mathrm{~d}, J=10.2 \mathrm{~Hz}), 125.0(\mathrm{~s}), 122.8$ $(\mathrm{d}, J=3.6 \mathrm{~Hz}), 61.8(\mathrm{~d}, J=6.1 \mathrm{~Hz}), 16.2(\mathrm{~d}, J=6.7 \mathrm{~Hz}) .{ }^{31} \mathrm{P}$ NMR $\left(162 \mathrm{MHz}, \mathrm{CDCl}_{3}\right)$ $\delta$ 28.6. HRMS (ESI) calcd for $\mathrm{C}_{15} \mathrm{H}_{16} \mathrm{~N}_{2} \mathrm{O}_{2} \mathrm{P}^{+}[\mathrm{M}+\mathrm{H}]^{+} m / z$ : 287.0944 ; found 287.0949.

\section{General Procedure for the Synthesis of Cyclic Imines $2^{6}$}

Cyclic imines 2 were synthesized according to the reported procedure. ${ }^{6} 2$ Aminophenol derivatives (5.5 mmol), 2-fluorobenzaldehyde derivatives (5 mmol), and $\mathrm{K}_{2} \mathrm{CO}_{3}(5 \mathrm{mmol}, 0.690 \mathrm{~g})$ were added into $20 \mathrm{~mL}$ of polyethylene glycol and the mixture was heated at $100{ }^{\circ} \mathrm{C}$ for $5 \mathrm{~h}$. After the reaction mixture was cooled to ambient temperature, $150 \mathrm{~mL}$ of water was added. The crude product was extracted with ethyl acetate, washed with brine twice, dried over anhydrous $\mathrm{Na}_{2} \mathrm{SO}_{4}$, and purified by column chromatography on silica gel $(\mathrm{PE}: \mathrm{EA}=10: 1, v / v)$. All cyclic imines 2 are known compounds.

Dibenzo $[b, f][1,4]$ oxazepine $(2 a)^{6}$<smiles>C1=Nc2ccccc2Oc2ccccc21</smiles>

Yellowish crystals (662 mg, 68\%); mp. 66-67 ${ }^{\circ} \mathrm{C}$ (Lit. ${ }^{7} \mathrm{mp} .68-69$ $\left.{ }^{\circ} \mathrm{C}\right) .{ }^{1} \mathrm{H}$ NMR $\left(400 \mathrm{MHz}, \mathrm{CDCl}_{3}\right) \delta 8.50(\mathrm{~s}, 1 \mathrm{H}), 7.43-7.34(\mathrm{~m}, 2 \mathrm{H}), 7.31-7.26(\mathrm{~m}$, 1H), $7.21-7.08(\mathrm{~m}, 5 \mathrm{H}) .{ }^{13} \mathrm{C} \mathrm{NMR}\left(101 \mathrm{MHz}, \mathrm{CDCl}_{3}\right) \delta 160.5,160.3,152.6,140.4$, $133.2,123.0,129.1,128.7,125.6,124.9,121.3,120.6$.

\section{7-Methyldibenzo $[b, f][1,4]$ oxazepine $(2 b)^{6}$}


<smiles>Cc1ccc2c(c1)Oc1ccccc1C=N2</smiles>

Brown crystals $(840 \mathrm{mg}, 81 \%)$; mp. $49-50{ }^{\circ} \mathrm{C}$ (Lit. ${ }^{8} \mathrm{mp} .44{ }^{\circ} \mathrm{C}$ ).

${ }^{1} \mathrm{H}$ NMR $\left(400 \mathrm{MHz}, \mathrm{CDCl}_{3}\right) \delta 8.45(\mathrm{~s}, 1 \mathrm{H}), 7.44-7.38(\mathrm{~m}, 1 \mathrm{H}), 7.30$ (dd, $J=7.6,1.6$ $\mathrm{Hz}, 1 \mathrm{H}), 7.24(\mathrm{~d}, J=8.0 \mathrm{~Hz}, 1 \mathrm{H}), 7.20-7.09(\mathrm{~m}, 2 \mathrm{H}), 6.99-6.91(\mathrm{~m}, 2 \mathrm{H}), 2.31(\mathrm{~s}$, $3 \mathrm{H}) .{ }^{13} \mathrm{C}$ NMR $\left(101 \mathrm{MHz}, \mathrm{CDCl}_{3}\right) \delta 160.2,159.7,152.2,139.3,137.9,133.1,130.0$, $128.9,127.3,126.3,124.9,121.7,120.6,20.8$

\section{7-Chlorodibenzo $[b, f][1,4]$ oxazepine $(2 c)^{9}$}<smiles>Clc1ccc2c(c1)Oc1ccccc1C=N2</smiles>

Beige solid (770 mg, 62\%); mp. 101-102 ${ }^{\circ} \mathrm{C}$ (Lit. ${ }^{8} \mathrm{mp} .94{ }^{\circ} \mathrm{C}$ ). ${ }^{1} \mathrm{H}$ NMR $\left(400 \mathrm{MHz}, \mathrm{CDCl}_{3}\right) \delta 8.49(\mathrm{~s}, 1 \mathrm{H}), 7.50-7.43(\mathrm{~m}, 1 \mathrm{H}), 7.34(\mathrm{dd}, J=7.6,1.6$ $\mathrm{Hz}, 1 \mathrm{H}), 7.30-7.20(\mathrm{~m}, 2 \mathrm{H}), 7.17-7.11(\mathrm{~m}, 3 \mathrm{H}) .{ }^{13} \mathrm{C}$ NMR $\left(101 \mathrm{MHz}, \mathrm{CDCl}_{3}\right) \delta$ $160.7,159.9,152.8,139.2,133.8,133.5,130.2,123.0,127.1,125.9,125.4,121.7,120.7$

\section{8-Methyldibenzo $[b, f][1,4]$ oxazepine $(2 \mathrm{~d})^{6}$}<smiles>Cc1ccc2c(c1)N=Cc1ccccc1O2</smiles>

Brown crystals $(820 \mathrm{mg}, 79 \%)$; mp. $50-51^{\circ} \mathrm{C}$ (Lit. $\left.{ }^{8} \mathrm{mp} .40{ }^{\circ} \mathrm{C}\right)$. ${ }^{1} \mathrm{H}$ NMR (400 MHz, $\left.\mathrm{CDCl}_{3}\right) \delta 8.50(\mathrm{~s}, 1 \mathrm{H}), 7.42$ (td, $\left.J=7.6,1.6 \mathrm{~Hz}, 1 \mathrm{H}\right), 7.31$ (dd, $J$ $=7.6,1.6 \mathrm{~Hz}, 1 \mathrm{H}), 7.21-7.09(\mathrm{~m}, 3 \mathrm{H}), 7.01(\mathrm{~d}, J=1.2 \mathrm{~Hz}, 2 \mathrm{H}), 2.30(\mathrm{~s}, 3 \mathrm{H}) .{ }^{13} \mathrm{C} \mathrm{NMR}$ $\left(101 \mathrm{MHz}, \mathrm{CDCl}_{3}\right) \delta 160.6,150.6,140.1,135.4,133.3,130.1,129.6,129.3,127.4$, $125.0,121.0,120.7,20.7$.

\section{8-Fluorodibenzo $[b, f][1,4]$ oxazepine $(2 \mathrm{e})^{6}$}

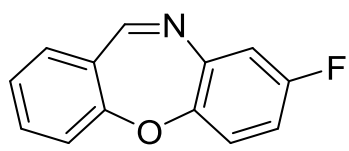

Beige crystals $(380 \mathrm{mg}, 36 \%)$; mp. $73-74{ }^{\circ} \mathrm{C}$ (Lit. ${ }^{6} \mathrm{mp} .74-75$ $\left.{ }^{\circ} \mathrm{C}\right) .{ }^{1} \mathrm{H} \mathrm{NMR}\left(400 \mathrm{MHz}, \mathrm{CDCl}_{3}\right) \delta 8.53(\mathrm{~s}, 1 \mathrm{H}), 7.46(\mathrm{td}, J=8.0,1.6 \mathrm{~Hz}, 1 \mathrm{H}), 7.35(\mathrm{dd}$, $J=7.6,1.2 \mathrm{~Hz}, 1 \mathrm{H}), 7.27-7.19(\mathrm{~m}, 1 \mathrm{H}), 7.13(\mathrm{~d}, J=8.0 \mathrm{~Hz}, 1 \mathrm{H}), 7.10-7.04(\mathrm{~m}, 2 \mathrm{H})$, $6.95-6.87(\mathrm{~m}, 1 \mathrm{H}) .{ }^{13} \mathrm{C} \mathrm{NMR}\left(101 \mathrm{MHz}, \mathrm{CDCl}_{3}\right) \delta 161.6,160.4,159.9(\mathrm{~d}, J=243.3$ 
Hz), 148.8 (d, $J=2.4 \mathrm{~Hz}), 141.5$ (d, $J=10.8 \mathrm{~Hz}), 133.6,130.2,127.1,125.2,122.0$ (d, $J=9.3 \mathrm{~Hz}), 120.6,115.3(\mathrm{~d}, J=24.1 \mathrm{~Hz}), 115.0(\mathrm{~d}, J=23.4 \mathrm{~Hz})$.

\section{8-Chlorodibenzo $[b, f][1,4]$ oxazepine $(2 \mathrm{f})^{6}$}<smiles>Clc1ccc2c(c1)N=Cc1ccccc1O2</smiles>

Yellowish crystals $(840 \mathrm{mg}, 73 \%)$; mp. $79-80{ }^{\circ} \mathrm{C}$ (Lit. ${ }^{6} \mathrm{mp} .75-$ $\left.76{ }^{\circ} \mathrm{C}\right) .{ }^{1} \mathrm{H} \mathrm{NMR}\left(400 \mathrm{MHz}, \mathrm{CDCl}_{3}\right) \delta 8.52(\mathrm{~s}, 1 \mathrm{H}), 7.46$ (td, $\left.J=7.6,1.6 \mathrm{~Hz}, 1 \mathrm{H}\right), 7.37$ - $7.32(\mathrm{~m}, 2 \mathrm{H}), 7.27-7.10(\mathrm{~m}, 3 \mathrm{H}), 7.05$ (d, $J=8.4 \mathrm{~Hz}, 1 \mathrm{H}) .{ }^{13} \mathrm{C}$ NMR (101 MHz, $\left.\mathrm{CDCl}_{3}\right) \delta 161.7,160.2,151.3,141.4,133.6,130.6,130.3,128.8,128.3,127.1,125.3$, $122.3,120.6$

\section{8-Bromodibenzo $[b, f][1,4]$ oxazepine $(2 \mathrm{~g})^{6}$}<smiles>Brc1ccc2c(c1)N=Cc1ccccc1O2</smiles>

Beige solid (940 mg, 58\%); mp. 97-98 ${ }^{\circ} \mathrm{C} .{ }^{1} \mathrm{H}$ NMR (400 MHz, $\left.\mathrm{CDCl}_{3}\right) \delta 8.51(\mathrm{~s}, 1 \mathrm{H}), 7.52-7.43(\mathrm{~m}, 2 \mathrm{H}), 7.37-7.28(\mathrm{~m}, 2 \mathrm{H}), 7.27-7.19(\mathrm{~m}, 1 \mathrm{H})$, $7.12(\mathrm{~d}, J=8.0 \mathrm{~Hz}, 1 \mathrm{H}), 6.99(\mathrm{~d}, J=8.4 \mathrm{~Hz}, 1 \mathrm{H}) .{ }^{13} \mathrm{C} \mathrm{NMR}\left(101 \mathrm{MHz}, \mathrm{CDCl}_{3}\right) \delta 161.7$, $160.2,151.9,141.7,133.6,131.8,131.3,130.3,127.1,125.3,122.8,120.6,118.0$.

\section{8-Methoxydibenzo $[b, f][1,4]$ oxazepine $(2 \mathrm{~h})^{9}$}<smiles>COc1ccc2c(c1)N=Cc1ccccc1O2</smiles>

Brown solid (790 mg, 70\%); mp. $70-71{ }^{\circ} \mathrm{C}$ (Lit. ${ }^{10} \mathrm{mp} .61-63$ $\left.{ }^{\circ} \mathrm{C}\right) .{ }^{1} \mathrm{H}$ NMR $\left(400 \mathrm{MHz}, \mathrm{CDCl}_{3}\right) \delta 8.52(\mathrm{~s}, 1 \mathrm{H}), 7.49-7.39(\mathrm{~m}, 1 \mathrm{H}), 7.33$ (dd, J= 7.6, $1.6 \mathrm{~Hz}, 1 \mathrm{H}), 7.19(\mathrm{td}, J=7.6,1.2 \mathrm{~Hz}, 1 \mathrm{H}), 7.13(\mathrm{~d}, J=8.0 \mathrm{~Hz}, 1 \mathrm{H}), 7.04(\mathrm{~d}, J=8.8 \mathrm{~Hz}$, $1 \mathrm{H}), 6.89(\mathrm{~d}, J=3.2 \mathrm{~Hz}, 1 \mathrm{H}), 6.76(\mathrm{dd}, J=8.8,2.8 \mathrm{~Hz}, 1 \mathrm{H}), 3.78(\mathrm{~s}, 3 \mathrm{H}) .{ }^{13} \mathrm{C} \mathrm{NMR}$ $\left(101 \mathrm{MHz}, \mathrm{CDCl}_{3}\right) \delta 161.0,160.7,157.1,146.4,140.9,133.3,130.1,127.3,124.9$, $121.7,120.5,114.5,113.0,55.6$.

\section{3-Methyldibenzo $[b, f][1,4]$ oxazepine $(2 \mathrm{i})^{9}$}


<smiles>Cc1ccc2c(c1)Oc1ccccc1N=C2</smiles>

Yellowish crystals $(691 \mathrm{mg}, 66 \%)$; mp. $87-88^{\circ} \mathrm{C}$ (Lit. $\left.{ }^{8} \mathrm{mp} .85^{\circ} \mathrm{C}\right)$.

${ }^{1} \mathrm{H}$ NMR (400 MHz, $\left.\mathrm{CDCl}_{3}\right) \delta 8.47(\mathrm{~s}, 1 \mathrm{H}), 7.38$ - $7.32(\mathrm{~m}, 1 \mathrm{H}), 7.24-7.13(\mathrm{~m}, 3 \mathrm{H})$, $7.13-7.08(\mathrm{~m}, 1 \mathrm{H}), 7.02-6.94(\mathrm{~m}, 2 \mathrm{H}), 2.36(\mathrm{~s}, 3 \mathrm{H}) .{ }^{13} \mathrm{C} \mathrm{NMR}\left(101 \mathrm{MHz}, \mathrm{CDCl}_{3}\right) \delta$ $160.6,160.3,152.7,144.6,140.6,130.0,129.2,128.6,125.8,125.6,124.7,121.3,121.2$, 21.4

\section{3-Chlorodibenzo $[b, f][1,4]$ oxazepine $(2 \mathrm{j})^{9}$}<smiles>Clc1ccc2c(c1)Oc1ccccc1N=C2</smiles>

Beige solid (778 mg, 68\%); mp. $111-112{ }^{\circ} \mathrm{C}$ (Lit. $\left.{ }^{11} \mathrm{mp} .112{ }^{\circ} \mathrm{C}\right)$. ${ }^{1} \mathrm{H}$ NMR $\left(400 \mathrm{MHz}, \mathrm{CDCl}_{3}\right) \delta 8.46(\mathrm{~s}, 1 \mathrm{H}), 7.36(\mathrm{dd}, J=7.6,2.0 \mathrm{~Hz}, 1 \mathrm{H}), 7.27-7.23$ $(\mathrm{m}, 2 \mathrm{H}), 7.23-7.20(\mathrm{~m}, 1 \mathrm{H}), 7.20-7.18(\mathrm{~m}, 1 \mathrm{H}), 7.18-7.15(\mathrm{~m}, 1 \mathrm{H}), 7.12-7.08(\mathrm{~m}$, 1H). ${ }^{13} \mathrm{C} \mathrm{NMR}\left(101 \mathrm{MHz}, \mathrm{CDCl}_{3}\right) \delta 160.7,159.4,152.2,140.2,139.0,130.8,129.4$, 129.0, 126.0, 125.8, 125.4, 121.3.

\section{2-Nitrodibenzo $[b, f][1,4]$ oxazepine $(2 k)^{12}$}<smiles>O=[N+]([O-])c1ccc2c(c1)C=Nc1ccccc1O2</smiles>

Yellow crystals $(696 \mathrm{mg}, 58 \%)$; mp. $231-232{ }^{\circ} \mathrm{C}$ (Lit. ${ }^{11} \mathrm{mp}$. $\left.230{ }^{\circ} \mathrm{C}\right) .{ }^{1} \mathrm{H} \mathrm{NMR}\left(400 \mathrm{MHz}, \mathrm{CDCl}_{3}\right) \delta 8.52(\mathrm{~s}, 1 \mathrm{H}), 8.32(\mathrm{dd}, J=8.8,2.8 \mathrm{~Hz}, 1 \mathrm{H})$, $8.25(\mathrm{~d}, J=2.8 \mathrm{~Hz}, 1 \mathrm{H}), 7.40(\mathrm{~m}, 1 \mathrm{H}), 7.32-7.21(\mathrm{~m}, 3 \mathrm{H}), 7.13(\mathrm{dd}, J=7.8,1.6 \mathrm{~Hz}$, 1H). ${ }^{13} \mathrm{C} \mathrm{NMR}\left(101 \mathrm{MHz}, \mathrm{CDCl}_{3}\right) \delta 165.0,158.1,151.6,144.9,139.6,129.7,128.4$, $127.6,126.6,125.8,122.0,121.4$.

\section{7-Nitrodibenzo $[b, f][1,4]$ oxazepine $(2 \mathrm{l})^{6}$}<smiles>O=[N+]([O-])c1ccc2c(c1)Oc1ccccc1C=N2</smiles>

Yellow needles (165 mg, 14\%); mp. $171-173{ }^{\circ} \mathrm{C} .{ }^{1} \mathrm{H}$ NMR (400 $\left.\mathrm{MHz}, \mathrm{CDCl}_{3}\right) \delta 8.64(\mathrm{~s}, 1 \mathrm{H}), 8.11-8.01(\mathrm{~m}, 2 \mathrm{H}), 7.56(\mathrm{td}, J=8.0,1.6 \mathrm{~Hz}, 1 \mathrm{H}), 7.49$ $(\mathrm{d}, J=8.8 \mathrm{~Hz}, 1 \mathrm{H}), 7.43(\mathrm{dd}, J=7.6,1.6 \mathrm{~Hz}, 1 \mathrm{H}), 7.34-7.28(\mathrm{~m}, 1 \mathrm{H}), 7.23(\mathrm{~d}, J=8.0$ 
$\mathrm{Hz}, 1 \mathrm{H}) .{ }^{13} \mathrm{C} \mathrm{NMR}\left(101 \mathrm{MHz}, \mathrm{CDCl}_{3}\right) \delta 163.3,159.9,152.7,147.1,146.1,134.3,130.5$, $129.5,126.9,125.9,120.9,120.8,117.4$.

\section{3,7-Dichlorodibenzo $[b, f][1,4]$ oxazepine $(2 \mathrm{~m})$}<smiles>Clc1ccc2c(c1)Oc1cc(Cl)ccc1N=C2</smiles>

Yellow needles (898 mg, 68\%); mp. $165-167 \quad{ }^{\circ} \mathrm{C}$

(Sublimating). ${ }^{1} \mathrm{H}$ NMR (400 MHz, $\left.\mathrm{CDCl}_{3}\right) \delta 8.43$ (s, 1H), $7.32-7.24$ (m, 2H), 7.22 $(\mathrm{dd}, J=8.2,1.9 \mathrm{~Hz}, 1 \mathrm{H}), 7.19-7.15(\mathrm{~m}, 2 \mathrm{H}), 7.12(\mathrm{~d}, J=2.3 \mathrm{~Hz}, 1 \mathrm{H}) .{ }^{13} \mathrm{C} \mathrm{NMR}(101$ $\left.\mathrm{MHz}, \mathrm{CDCl}_{3}\right) \delta 160.2,159.6,152.3,139.4,138.9,134.2,131.0,130.1,126.3,125.8$, 125.6, 121.8, 121.4. HRMS (ESI) calcd for $\mathrm{C}_{13} \mathrm{H}_{8} \mathrm{Cl}_{2} \mathrm{NO}^{+}[\mathrm{M}+\mathrm{H}]^{+} m / z: 263.9977$; found 263.9974 .

\section{3,7-Dimethyldibenzo $[b, f][1,4]$ oxazepine $(2 \mathrm{n})^{12}$}

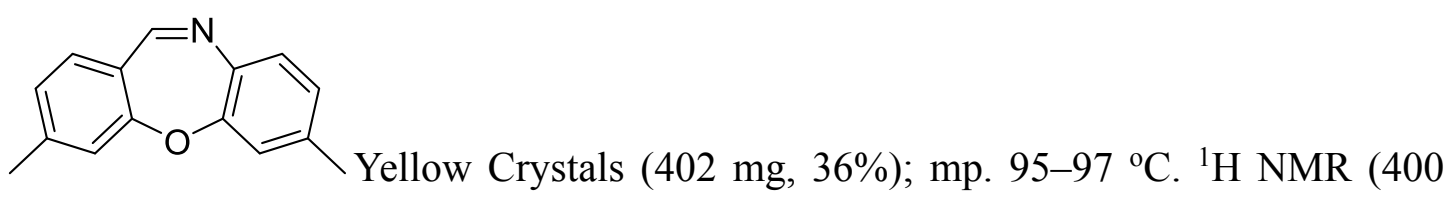
$\left.\mathrm{MHz}, \mathrm{CDCl}_{3}\right) \delta 8.41(\mathrm{~s}, 1 \mathrm{H}), 7.31-7.13(\mathrm{~m}, 2 \mathrm{H}), 7.04-6.90(\mathrm{~m}, 4 \mathrm{H}), 2.36(\mathrm{~s}, 3 \mathrm{H})$, $2.32(\mathrm{~s}, 3 \mathrm{H}) .{ }^{13} \mathrm{C} \mathrm{NMR}\left(101 \mathrm{MHz}, \mathrm{CDCl}_{3}\right) \delta 160.2,159.8,152.3,144.4,139.2,138.1$, $130.0,129.0,126.3,125.7,124.8,121.8,121.2,21.4,20.8$.

\section{Dibenzo $[b, f][1,4]$ thiazepine $(20)^{12}$}<smiles>C1=Nc2ccccc2Sc2ccccc21</smiles>
Purple black crystals (254 mg, 24\%); mp. 108-109 ${ }^{\circ} \mathrm{C}$ (Lit. ${ }^{13} \mathrm{mp}$. $\left.115-116{ }^{\circ} \mathrm{C}\right) .{ }^{1} \mathrm{H}$ NMR $\left(400 \mathrm{MHz}, \mathrm{CDCl}_{3}\right) \delta 8.89(\mathrm{~s}, 1 \mathrm{H}), 7.51-7.27(\mathrm{~m}, 7 \mathrm{H}), 7.19-$ $7.12(\mathrm{~m}, 1 \mathrm{H}) .{ }^{13} \mathrm{C} \mathrm{NMR}\left(101 \mathrm{MHz}, \mathrm{CDCl}_{3}\right) \delta 162.2,148.5,139.3,137.2,132.7,131.6$, $131.4,129.4,129.2,128.8,128.2,127.2,126.9$.

\section{3-Methyldibenzo $[b, f][1,4]$ thiazepine $(2 p)^{12}$}


<smiles>Cc1ccc2c(c1)Sc1ccccc1N=C2</smiles>

Yellow crystals (530 mg, 47\%); mp. 58-59 ${ }^{\circ} \mathrm{C}$ (Lit. ${ }^{14} \mathrm{mp} .60-61^{\circ} \mathrm{C}$ ).

${ }^{1} \mathrm{H}$ NMR $\left(400 \mathrm{MHz}, \mathrm{CDCl}_{3}\right) \delta 8.83(\mathrm{~s}, 1 \mathrm{H}), 7.39(\mathrm{dt}, J=8.0,0.8 \mathrm{~Hz}, 1 \mathrm{H}), 7.31-7.27$ (m, 2H), $7.25-7.21(\mathrm{~m}, 2 \mathrm{H}), 7.16-7.10(\mathrm{~m}, 2 \mathrm{H}), 2.30(\mathrm{~s}, 3 \mathrm{H}) .{ }^{13} \mathrm{C}$ NMR (101 MHz, $\left.\mathrm{CDCl}_{3}\right) \delta 162.3,148.7,142.3,139.1,134.6,132.7,132.1,129.5,129.2,129.0,128.9$, $127.1,127.0,21.2$

\section{4-Fluorodibenzo $[b, f][1,4]$ thiazepine $(2 q)^{12}$}<smiles>Fc1cccc2c1Sc1ccccc1N=C2</smiles>

Purple crystals (295 mg, 26\%); mp. 93-94 ${ }^{\circ} \mathrm{C} .{ }^{1} \mathrm{H}$ NMR $(400 \mathrm{MHz}$, $\left.\mathrm{CDCl}_{3}\right) \delta 8.90(\mathrm{~d}, J=1.6 \mathrm{~Hz}, 1 \mathrm{H}), 7.48(\mathrm{dd}, J=8.0,1.6 \mathrm{~Hz}, 1 \mathrm{H}), 7.38-7.27(\mathrm{~m}, 3 \mathrm{H})$, $7.21-7.14(\mathrm{~m}, 3 \mathrm{H}) .{ }^{19} \mathrm{~F}$ NMR $\left(377 \mathrm{MHz}, \mathrm{CDCl}_{3}\right) \delta-110.42 .{ }^{13} \mathrm{C}$ NMR $(101 \mathrm{MHz}$, $\left.\mathrm{CDCl}_{3}\right) \delta 161.2(\mathrm{~d}, J=2.0 \mathrm{~Hz}), 159.8(\mathrm{~d}, J=247.4 \mathrm{~Hz}), 148.9,139.1,133.3,129.6$, $129.4(\mathrm{~d}, J=8.2 \mathrm{~Hz}), 127.7,127.4,126.9,126.6(\mathrm{~d}, J=20.5 \mathrm{~Hz}), 124.7(\mathrm{~d}, J=3.7 \mathrm{~Hz})$, $117.8(\mathrm{~d}, J=23.4 \mathrm{~Hz})$.

\section{8-Chlorodibenzo $[b, f][1,4]$ thiazepine $(2 r)^{12}$}<smiles>Clc1ccc2c(c1)N=Cc1ccccc1S2</smiles>

Yellowish crystals $(770 \mathrm{mg}, 62 \%)$; $\mathrm{mp} .76-77^{\circ} \mathrm{C}$ (Lit. ${ }^{15} \mathrm{mp} .78-$ $\left.79^{\circ} \mathrm{C}\right) .{ }^{1} \mathrm{H}$ NMR $\left(400 \mathrm{MHz}, \mathrm{CDCl}_{3}\right) \delta 8.88(\mathrm{~s}, 1 \mathrm{H}), 7.44-7.27(\mathrm{~m}, 6 \mathrm{H}), 7.11(\mathrm{dd}, J=$ 8.0, $2.4 \mathrm{~Hz}, 1 \mathrm{H}) .{ }^{13} \mathrm{C} \mathrm{NMR}\left(101 \mathrm{MHz}, \mathrm{CDCl}_{3}\right) \delta 163.2,149.4,139.0,137.0,134.9$, $133.5,131.7,131.6,129.4,128.4,127.4,127.0,126.6$.

\section{1-Methyldibenzo $[b, f][1,4]$ oxazepine $(2 s)^{16}$}<smiles>CC1=Nc2ccccc2Oc2ccccc21</smiles>

Yellow crystals (899 mg, 86\%), mp. $81-83{ }^{\circ} \mathrm{C}$ (Lit. ${ }^{17} \mathrm{mp} .82-84{ }^{\circ} \mathrm{C}$ ). ${ }^{1} \mathrm{H} \mathrm{NMR}\left(400 \mathrm{MHz}, \mathrm{CDCl}_{3}\right) \delta 7.41(\mathrm{dd}, J=15.8,7.9 \mathrm{~Hz}, 2 \mathrm{H}), 7.29-7.26(\mathrm{~m}, 1 \mathrm{H}), 7.20$ 
$7.16(\mathrm{~m}, 2 \mathrm{H}), 7.15-7.13(\mathrm{~m}, 3 \mathrm{H}), 2.63(\mathrm{~s}, 3 \mathrm{H}) .{ }^{13} \mathrm{C} \mathrm{NMR}\left(100 \mathrm{MHz}, \mathrm{CDCl}_{3}\right) \delta 167.5$, 161.0, 152.7, 140.8, 132.9, 129.2, 128.6, 127.8, 127.4, 125.7, 125.2, 121.0, 120.8, 27.8.

\section{1-Methyldibenzo $[b, f][1,4]$ oxazepine $(2 t)^{16}$}<smiles>c1ccc(C2=Nc3ccccc3Oc3ccccc32)cc1</smiles>

Yellow crystals $(881 \mathrm{mg}, 65 \%), \mathrm{mp} .104-106{ }^{\circ} \mathrm{C}$ (Lit. ${ }^{17} \mathrm{mp} .105-$ $\left.107^{\circ} \mathrm{C}\right) .{ }^{1} \mathrm{H}$ NMR $\left(400 \mathrm{MHz}, \mathrm{CDCl}_{3}\right) \delta 7.82(\mathrm{dd}, J=7.7,1.4 \mathrm{~Hz}, 2 \mathrm{H}), 7.47-7.42(\mathrm{~m}$, 5H), 7.33-7.27 (m, 1H), 7.19-7.12 (m, 5H). $\left.{ }^{13} \mathrm{C} \mathrm{NMR} \mathrm{(100} \mathrm{MHz,} \mathrm{CDCl}_{3}\right) \delta 167.3$, $162.2,152.6,141.1,140.3,133.2,131.5,130.6,129.9,128.4,127.7,127.6,125.8,124.7$, 121.2, 120.9.

\section{General Procedure for the Synthesis of Products 3.}

Diazo compound $1(0.4 \mathrm{mmol})$ and cyclic imine $2(0.2 \mathrm{mmol})$ were added into a 8 $\mathrm{mL}$ microwave reaction tube. The tube was charged with $\mathrm{N}_{2}$ and added $3 \mathrm{~mL}$ of dry $\mathrm{PhCl}$, and then the resulting mixture was stirred in a microwave reactor at $160{ }^{\circ} \mathrm{C}$ for $10 \mathrm{~min}$. After cooling to room temperature, $\mathrm{PhCl}$ was removed and the residue was directly subjected to flash column chromatography (PE:EA $=5: 1$ to $1: 1, v / v)$ to afford product 3 .

\section{Rel-(4bS,15S,16S)-15,16-diphenyl-4b,16-dihydrodibenzo[b,f]benzo[4,5][1,2] aza-} phosphinino[1,6-d][1,4]oxazepine 15-oxide (3a)<smiles></smiles>

White solid (82 mg, 84\%); mp. 286-288 ${ }^{\circ} \mathrm{C}$. IR (KBr) $v\left(\mathrm{~cm}^{-1}\right) 3057$, 2924, 2851, 1599, 1492, 1258, 1243, 1210, 1116, 1049, 1017, 978, 784, 751, 693. ${ }^{1} \mathrm{H}$ NMR (400 MHz, $\left.\mathrm{CDCl}_{3}\right) \delta 7.77(\mathrm{dd}, J=7.0,1.9 \mathrm{~Hz}, 1 \mathrm{H}), 7.45-7.32(\mathrm{~m}, 3 \mathrm{H}), 7.27-$ $7.02(\mathrm{~m}, 13 \mathrm{H}), 6.80-6.62(\mathrm{~m}, 5 \mathrm{H}), 6.34(\mathrm{~d}, J=7.8 \mathrm{~Hz}, 1 \mathrm{H}), 5.12(\mathrm{~d}, J=28.6 \mathrm{~Hz}, 1 \mathrm{H})$. 
${ }^{13} \mathrm{C}$ NMR $\left(101 \mathrm{MHz}, \mathrm{CDCl}_{3}\right) \delta 159.5,153.4(\mathrm{~d}, J=4.9 \mathrm{~Hz}), 140.4(\mathrm{~d}, J=3.8 \mathrm{~Hz}), 134.5$ (d, $J=3.0 \mathrm{~Hz}), 134.3$ (d, $J=5.1 \mathrm{~Hz}), 133.5$ (d, $J=9.0 \mathrm{~Hz}), 131.9$ (d, $J=2.0 \mathrm{~Hz}), 131.8$ (d, $J=4.1 \mathrm{~Hz}), 131.3$ (d, $J=5.3 \mathrm{~Hz}), 130.7,128.5$ (d, $J=153.8 \mathrm{~Hz}), 128.2,127.9$ (d, $J$ $=7.8 \mathrm{~Hz}), 127.8,127.5,126.9,126.1$ (d, $J=2.9 \mathrm{~Hz}), 126.0,125.6,125.3,125.0,121.0$, 120.6, $62.1(\mathrm{~d}, J=2.6 \mathrm{~Hz}), 51.4(\mathrm{~d}, J=78.6 \mathrm{~Hz}) .{ }^{31} \mathrm{P} \mathrm{NMR}\left(162 \mathrm{MHz}, \mathrm{CDCl}_{3}\right) \delta 28.6$. HRMS (ESI) calcd for $\mathrm{C}_{32} \mathrm{H}_{25} \mathrm{NO}_{2} \mathrm{P}^{+}[\mathrm{M}+\mathrm{H}]^{+} m / z$ : 486.1617; found 486.1623.

Rel-(4bS,15S,16S)-11-methyl-15,16-diphenyl-4b,16-dihydrodibenzo[b, $f]$ benzo[4,5] $[1,2]$ azaphosphinino[1,6-d][1,4]oxazepine 15-oxide (3b)

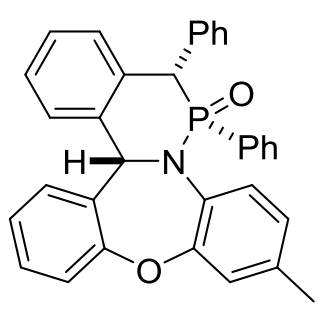

White solid (96 mg, 94\%); mp. $294-296{ }^{\circ} \mathrm{C} . \mathrm{IR}(\mathrm{KBr}) v\left(\mathrm{~cm}^{-1}\right)$ 3060, 2923, 2854, 1599, 1502, 1260, 1212, 1122, 980, 933, 785, 748, 695. ${ }^{1} \mathrm{H}$ NMR $\left(400 \mathrm{MHz}, \mathrm{CDCl}_{3}\right) \delta 7.65(\mathrm{~d}, J=8.0 \mathrm{~Hz}, 1 \mathrm{H}), 7.43-7.08(\mathrm{~m}, 15 \mathrm{H}), 6.86(\mathrm{~d}, J=1.3$ $\mathrm{Hz}, 1 \mathrm{H}), 6.75-6.65(\mathrm{~m}, 3 \mathrm{H}), 6.56(\mathrm{dd}, J=8.2,1.4 \mathrm{~Hz}, 1 \mathrm{H}), 6.30(\mathrm{~d}, J=7.6 \mathrm{~Hz}, 1 \mathrm{H})$, $5.07(\mathrm{~d}, J=28.2 \mathrm{~Hz}, 1 \mathrm{H}), 2.08(\mathrm{~s}, 3 \mathrm{H}) .{ }^{13} \mathrm{C} \mathrm{NMR}\left(101 \mathrm{MHz}, \mathrm{CDCl}_{3}\right) \delta 159.5,153.3(\mathrm{~d}$, $J=5.0 \mathrm{~Hz}), 140.4(\mathrm{~d}, J=4.2 \mathrm{~Hz}), 135.2,134.3(\mathrm{~d}, J=5.2 \mathrm{~Hz}), 133.6(\mathrm{~d}, J=9.0 \mathrm{~Hz})$, $132.0(\mathrm{~d}, J=4.2 \mathrm{~Hz}), 131.9(\mathrm{~d}, J=2.0 \mathrm{~Hz}), 131.8(\mathrm{~d}, J=2.4 \mathrm{~Hz}), 131.3$ (d, $J=5.2 \mathrm{~Hz})$, 131.6, 130.6, 128.7 (d, $J=130.1 \mathrm{~Hz}), 128.1,128.0(\mathrm{~d}, J=7.3 \mathrm{~Hz}), 127.8,127.5$ (d, $J=$ $2.0 \mathrm{~Hz}), 126.9,126.2,125.9,125.5,121.2,121.1,62.3(\mathrm{~d}, J=2.8 \mathrm{~Hz}), 51.5$ (d, $J=78.7$ $\mathrm{Hz}), 20.6 .{ }^{31} \mathrm{P} \mathrm{NMR}\left(162 \mathrm{MHz}, \mathrm{CDCl}_{3}\right) \delta 28.1$. HRMS (ESI) calcd for $\mathrm{C}_{33} \mathrm{H}_{27} \mathrm{NO}_{2} \mathrm{P}^{+}$ $[\mathrm{M}+\mathrm{H}]^{+} \mathrm{m} / z: 500.1774$; found: 500.1770.

Rel-(4bS,15S,16S)-11-chloro-15,16-diphenyl-4b,16-dihydrodibenzo[b,f]benzo[4,5] $[1,2]$ azaphosphinino[1,6-d][1,4] oxazepine 15-oxide (3c) 


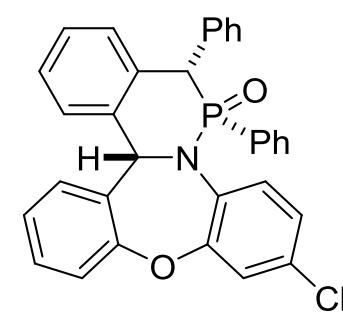

White solid (100 mg, 96\%); mp. 282-284 ${ }^{\circ} \mathrm{C}$. IR (KBr) $v\left(\mathrm{~cm}^{-1}\right)$ 3062, 2926, 2854, 1736, 1598, 1565, 1485, 1268, 1198, 1115, 1020, 975, 902, 782, 740, 701, 574, 528. ${ }^{1} \mathrm{H}$ NMR (400 MHz, $\left.\mathrm{CDCl}_{3}\right) \delta 7.65(\mathrm{~d}, J=8.8 \mathrm{~Hz}, 1 \mathrm{H}), 7.40-7.34(\mathrm{~m}$, 1H), $7.32-7.21(\mathrm{~m}, 3 \mathrm{H}), 7.19-7.10(\mathrm{~m}, 4 \mathrm{H}), 7.08-6.99(\mathrm{~m}, 7 \mathrm{H}), 6.97(\mathrm{~d}, J=2.4 \mathrm{~Hz}$, $1 \mathrm{H}), 6.71-6.60(\mathrm{~m}, 3 \mathrm{H}), 6.59-6.52(\mathrm{~m}, 1 \mathrm{H}), 6.25(\mathrm{~d}, J=8.0 \mathrm{~Hz}, 1 \mathrm{H}), 5.05(\mathrm{~d}, J=$ $28.7 \mathrm{~Hz}, 1 \mathrm{H}) .{ }^{13} \mathrm{C} \mathrm{NMR}\left(101 \mathrm{MHz}, \mathrm{CDCl}_{3}\right) \delta 159.2,153.7$ (d, $\left.J=5.0 \mathrm{~Hz}\right), 140.3$ (d, $J$ $=3.9 \mathrm{~Hz}), 134.2(\mathrm{~d}, J=5.3 \mathrm{~Hz}), 133.4(\mathrm{~d}, J=6.3 \mathrm{~Hz}), 133.4,132.2(\mathrm{~d}, J=2.8 \mathrm{~Hz})$, 131.8, 131.44, 131.35 (d, $J=5.7 \mathrm{~Hz}), 131.0,129.2,128.24$ (d, $J=2.2 \mathrm{~Hz}), 128.20$ (d, $J=142.6 \mathrm{~Hz}), 128.1,127.7(\mathrm{~d}, J=2.7 \mathrm{~Hz}), 127.4(\mathrm{~d}, J=2.2 \mathrm{~Hz}), 127.4,127.0(\mathrm{~d}, J=$ $2.0 \mathrm{~Hz}), 126.7(\mathrm{~d}, J=3.4 \mathrm{~Hz}), 126.0,125.8(\mathrm{~d}, J=2.3 \mathrm{~Hz}), 125.4,121.2,121.0,62.0$ $(\mathrm{d}, J=3.1 \mathrm{~Hz}), 51.4(\mathrm{~d}, J=78.5 \mathrm{~Hz}) .{ }^{31} \mathrm{P} \mathrm{NMR}\left(162 \mathrm{MHz}, \mathrm{CDCl}_{3}\right) \delta 28.9$. HRMS (ESI) calcd for $\mathrm{C}_{32} \mathrm{H}_{24} \mathrm{ClNO}_{2} \mathrm{P}^{+}[\mathrm{M}+\mathrm{H}]^{+} m / z$ : 520.1228; found 520.1225.

Rel-(4bS,15S,16S)-12-methyl-15,16-diphenyl-4b,16-dihydrodibenzo[b, $f]$ benzo $[4,5]$ $[1,2]$ azaphosphinino[1,6-d][1,4]oxazepine 15-oxide (3d)

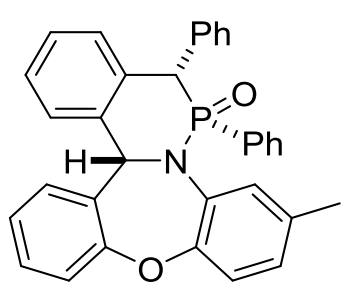

The crude residue was purified by flash chromatography (PE:EA $=4: 1$ to $1: 1, v / v)$ over silica gel. White solid $(88 \mathrm{mg}, 88 \%) ; \mathrm{mp} .300-302{ }^{\circ} \mathrm{C}$. IR $(\mathrm{KBr})$ $v\left(\mathrm{~cm}^{-1}\right) 2972,2926,1508,1380,1266,1214,1089,1048,880,744 .{ }^{1} \mathrm{H}$ NMR (400 MHz, $\left.\mathrm{CDCl}_{3}\right) \delta 7.56(\mathrm{~s}, 1 \mathrm{H}), 7.43-7.07(\mathrm{~m}, 15 \mathrm{H}), 6.91(\mathrm{~d}, J=8.1 \mathrm{~Hz}, 1 \mathrm{H}), 6.78-6.67(\mathrm{~m}$, $3 \mathrm{H}), 6.55(\mathrm{~d}, J=8.1 \mathrm{~Hz}, 1 \mathrm{H}), 6.30(\mathrm{~d}, J=7.7 \mathrm{~Hz}, 1 \mathrm{H}), 5.08(\mathrm{~d}, J=28.4 \mathrm{~Hz}, 1 \mathrm{H}) .{ }^{13} \mathrm{C}$ NMR (101 MHz, $\left.\mathrm{CDCl}_{3}\right) \delta 159.6,151.3(\mathrm{~d}, J=4.9 \mathrm{~Hz}), 140.3(\mathrm{~d}, J=4.2 \mathrm{~Hz}), 135.1$, $134.3(\mathrm{~d}, J=5.3 \mathrm{~Hz}), 134.1$ (d, $J=2.7 \mathrm{~Hz}), 133.5$ (d, $J=9.1 \mathrm{~Hz}), 131.9,131.3$ (d, $J=$ $5.3 \mathrm{~Hz}), 131.1$ (d, $J=89.6 \mathrm{~Hz}), 128.6$ (d, $J=153.7 \mathrm{~Hz}), 128.1,128.0,127.9,127.7$, $127.5(\mathrm{~d}, J=1.8 \mathrm{~Hz}), 126.9,126.6(\mathrm{~d}, J=2.8 \mathrm{~Hz}), 126.2,125.5,121.0,120.2,62.2(\mathrm{~d}$, 
$J=2.7 \mathrm{~Hz}), 51.5(\mathrm{~d}, J=78.7 \mathrm{~Hz}), 20.6 .{ }^{31} \mathrm{P} \mathrm{NMR}\left(162 \mathrm{MHz}, \mathrm{CDCl}_{3}\right) \delta 28.3 . \mathrm{HRMS}$ (ESI) calcd for $\mathrm{C}_{33} \mathrm{H}_{27} \mathrm{NO}_{2} \mathrm{P}^{+}[\mathrm{M}+\mathrm{H}]^{+} m / z$ : 500.1774; found 500.1775 .

Rel-(4bS,15S,16S)-12-fluoro-15,16-diphenyl-4b,16-dihydrodibenzo[b, $f]$ benzo $[4,5]$ $[1,2]$ azaphosphinino[1,6-d][1,4] oxazepine 15-oxide (3e)

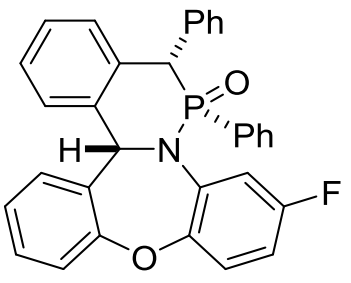

The crude residue was purified by flash chromatography (PE:EA

$=4: 1$ to $1: 1, v / v)$ over silica gel. White solid $(82 \mathrm{mg}, 81 \%)$; mp. $310-312^{\circ} \mathrm{C}$. IR $(\mathrm{KBr})$ $v\left(\mathrm{~cm}^{-1}\right) 3060,2925,2853,1736,1590,1517,1491,1340,1259,1243,1211,1113,974$, 745, 700. ${ }^{1} \mathrm{H}$ NMR $\left(400 \mathrm{MHz}, \mathrm{CDCl}_{3}\right) \delta 7.53(\mathrm{dd}, J=10.1,2.7 \mathrm{~Hz}, 1 \mathrm{H}), 7.49-7.43$ (m, 1H), $7.41-7.09(\mathrm{~m}, 14 \mathrm{H}), 6.98(\mathrm{dd}, J=8.8,5.5 \mathrm{~Hz}, 1 \mathrm{H}), 6.79(\mathrm{~d}, J=7.1 \mathrm{~Hz}, 2 \mathrm{H})$, $6.65-6.60(\mathrm{~m}, 1 \mathrm{H}), 6.47-6.39(\mathrm{~m}, 1 \mathrm{H}), 6.34(\mathrm{~d}, J=8.2 \mathrm{~Hz}, 1 \mathrm{H}), 5.13(\mathrm{~d}, J=29.0 \mathrm{~Hz}$, 1H). ${ }^{13} \mathrm{C}$ NMR $\left(101 \mathrm{MHz}, \mathrm{CDCl}_{3}\right) \delta 159.80,159.1\left(\mathrm{~d}, J_{C-F}=243.8 \mathrm{~Hz}\right), 149.7(\mathrm{dd}, J=$ 4.9, 3.1 Hz), 140.5 (d, $J=3.6 \mathrm{~Hz}), 135.6$ (d, $J=3.3 \mathrm{~Hz}), 135.5$ (d, $J=3.3 \mathrm{~Hz}), 134.2$ (d, $J=5.1 \mathrm{~Hz}), 133.4$ (d, $J=9.0 \mathrm{~Hz}), 128.33$ (d, $J=2.0 \mathrm{~Hz}), 128.25$ (d, $J=128.2 \mathrm{~Hz})$, 128.1, $128.1(\mathrm{~d}, J=0.6 \mathrm{~Hz}), 128.0,127.8(\mathrm{~d}, J=2.7 \mathrm{~Hz}), 127.4(\mathrm{~d}, J=6.6 \mathrm{~Hz}), 127.2$ $(\mathrm{d}, J=6.8 \mathrm{~Hz}), 127.1$ (d, $J=1.8 \mathrm{~Hz}), 126.0,125.6(\mathrm{~d}, J=2.1 \mathrm{~Hz}), 121.2(\mathrm{~d}, J=9.8 \mathrm{~Hz})$, 120.8, $113.0(\mathrm{~d}, J=23.0 \mathrm{~Hz}), 110.9(\mathrm{~d}, J=23.2 \mathrm{~Hz}), 61.9$ (d, $J=3.1 \mathrm{~Hz}), 51.3$ (d, $J=$ $78.3 \mathrm{~Hz}) .{ }^{31} \mathrm{P} \mathrm{NMR}\left(162 \mathrm{MHz}, \mathrm{CDCl}_{3}\right) \delta 29.1 .{ }^{19} \mathrm{~F} \mathrm{NMR}\left(376 \mathrm{MHz}, \mathrm{CDCl}_{3}\right) \delta-115.7$. HRMS (ESI) calcd for $\mathrm{C}_{32} \mathrm{H}_{24} \mathrm{FNO}_{2} \mathrm{P}^{+}[\mathrm{M}+\mathrm{H}]^{+} m / z$ : 504.1523; found 504.1524.

Rel-(4bS,15S,16S)-12-chloro-15,16-diphenyl-4b,16-dihydrodibenzo[b,f]benzo[4,5] $[1,2]$ azaphosphinino $[1,6-d][1,4]$ oxazepine 15-oxide (3f)

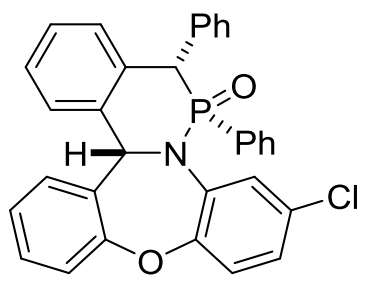

White solid (90 mg, 87\%); mp. $320-322^{\circ} \mathrm{C}$. IR (KBr) $v\left(\mathrm{~cm}^{-1}\right)$ 3060, 2926, 2852, 1598, 1492, 1241, 1210, 1111, 1076, 978, 782, 696. ${ }^{1} \mathrm{H}$ NMR (400 


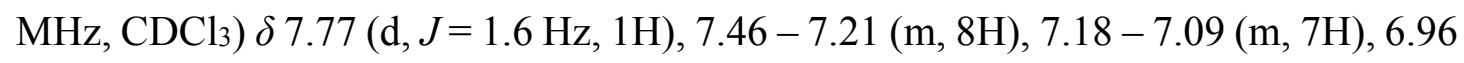
$(\mathrm{d}, J=8.4 \mathrm{~Hz}, 1 \mathrm{H}), 6.78(\mathrm{~d}, J=7.4 \mathrm{~Hz}, 2 \mathrm{H}), 6.73-6.68(\mathrm{~m}, 1 \mathrm{H}), 6.68-6.63(\mathrm{~m}, 1 \mathrm{H})$, $6.32(\mathrm{~d}, J=8.0 \mathrm{~Hz}, 1 \mathrm{H}), 5.12(\mathrm{~d}, J=28.9 \mathrm{~Hz}, 1 \mathrm{H}) .{ }^{13} \mathrm{C} \mathrm{NMR}\left(101 \mathrm{MHz}, \mathrm{CDCl}_{3}\right) \delta$ 159.3, $152.0(\mathrm{~d}, J=4.9 \mathrm{~Hz}), 140.2(\mathrm{~d}, J=3.5 \mathrm{~Hz}), 135.6(\mathrm{~d}, J=2.7 \mathrm{~Hz}), 134.2(\mathrm{~d}, J=$ $5.1 \mathrm{~Hz}), 133.4$ (d, $J=9.0 \mathrm{~Hz}), 132.3,131.8,131.4$ (d, $J=5.2 \mathrm{~Hz}), 131.0,130.2,128.3$, $128.22(\mathrm{~d}, J=128.2 \mathrm{~Hz}), 128.15(\mathrm{~d}, J=2.9 \mathrm{~Hz}), 128.0,127.7(\mathrm{~d}, J=2.0 \mathrm{~Hz}), 127.5$ (d, $J=3.0 \mathrm{~Hz}), 127.1,126.0,125.8,125.8$ (d, $J=3.2 \mathrm{~Hz}), 124.7,121.5,120.9,62.0$ (d, $J$ $=2.5 \mathrm{~Hz}), 51.4(\mathrm{~d}, J=78.5 \mathrm{~Hz}) .{ }^{31} \mathrm{P}$ NMR $\left(162 \mathrm{MHz}, \mathrm{CDCl}_{3}\right) \delta 29.0$. HRMS (ESI) calcd for $\mathrm{C}_{32} \mathrm{H}_{24} \mathrm{ClNO}_{2} \mathrm{P}^{+}[\mathrm{M}+\mathrm{H}]^{+} m / z$ : 520.1228; found 520.1231.

\section{Rel-(4bS,15S,16S)-12-bromo-15,16-diphenyl-4b,16-dihydrodibenzo[b, $f]$ benzo[4,5]} $[1,2]$ azaphosphinino[1,6-d][1,4] oxazepine 15-oxide (3g)

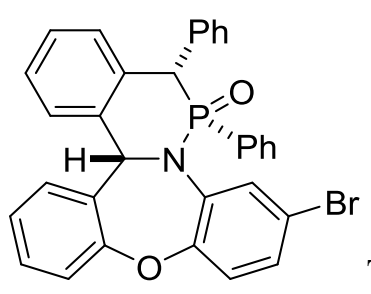

The crude residue was purified by flash chromatography (PE:EA $=4: 1$ to $1: 1, v / v)$ over silica gel. White solid (94 mg, 83\%); mp. 330-332 ${ }^{\circ} \mathrm{C}$. IR (KBr) $v\left(\mathrm{~cm}^{-1}\right) 2974,2928,1619,1591,1483,1458,1326,1213,1089,1049,978,881,738$, 701. ${ }^{1} \mathrm{H}$ NMR $\left(400 \mathrm{MHz}, \mathrm{CDCl}_{3}\right) \delta 7.90(\mathrm{~d}, J=1.4 \mathrm{~Hz}, 1 \mathrm{H}), 7.49-7.40(\mathrm{~m}, 1 \mathrm{H}), 7.39$ $-7.11(\mathrm{~m}, 14 \mathrm{H}), 6.92-6.82(\mathrm{~m}, 2 \mathrm{H}), 6.78(\mathrm{~d}, J=7.4 \mathrm{~Hz}, 2 \mathrm{H}), 6.70-6.62(\mathrm{~m}, 1 \mathrm{H})$, $6.31(\mathrm{~d}, J=8.0 \mathrm{~Hz}, 1 \mathrm{H}), 5.11(\mathrm{~d}, J=28.9 \mathrm{~Hz}, 1 \mathrm{H}) .{ }^{13} \mathrm{C} \mathrm{NMR}\left(101 \mathrm{MHz}, \mathrm{CDCl}_{3}\right) \delta$ $159.2,152.5(\mathrm{~d}, J=4.9 \mathrm{~Hz}), 140.2(\mathrm{~d}, J=3.8 \mathrm{~Hz}), 135.9$ (d, $J=2.9 \mathrm{~Hz}), 134.2$ (d, $J=$ $5.2 \mathrm{~Hz}), 133.4$ (d, $J=9.1 \mathrm{~Hz}), 132.3,131.8(\mathrm{~d}, J=2.0 \mathrm{~Hz}), 131.4(\mathrm{~d}, J=5.4 \mathrm{~Hz}), 131.0$, $128.6(\mathrm{~d}, J=3.1 \mathrm{~Hz}), 128.28,128.23(\mathrm{~d}, J=128.2 \mathrm{~Hz}), 128.2(\mathrm{~d}, J=4.5 \mathrm{~Hz}), 128.0$, 127.7, $127.5(\mathrm{~d}, J=6.8 \mathrm{~Hz}), 127.4,127.1,126.0$ (d, $J=12.7 \mathrm{~Hz}), 122.0,121.0,117.7$, $62.0(\mathrm{~d}, J=2.4 \mathrm{~Hz}), 51.4(\mathrm{~d}, J=78.5 \mathrm{~Hz}) .{ }^{31} \mathrm{P} \mathrm{NMR}\left(162 \mathrm{MHz}, \mathrm{CDCl}_{3}\right) \delta 29.0$. HRMS (ESI) calcd for $\mathrm{C}_{32} \mathrm{H}_{24} \mathrm{BrNO}_{2} \mathrm{P}^{+}[\mathrm{M}+\mathrm{H}]^{+} \mathrm{m} / z$ : 564.0723; found 564.0728.

\section{Rel-(4bS,15S,16S)-12-methoxy-15,16-diphenyl-4b,16-dihydrodibenzo $[b, f]$ benzo} $[4,5][1,2]$ azaphosphinino $[1,6-d][1,4]$ oxazepine 15-oxide (3h) 


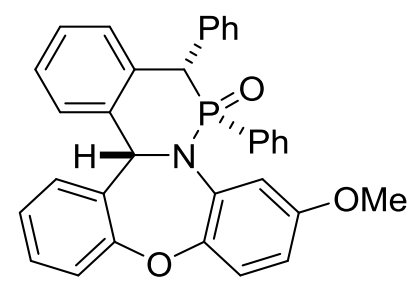

The crude residue was purified by flash chromatography (PE:EA $=3: 1$ to $1: 1, v / v)$ over silica gel. White solid (73 mg, 71\%); mp. 286-288 ${ }^{\circ} \mathrm{C}$. IR $(\mathrm{KBr}) v\left(\mathrm{~cm}^{-1}\right)$ 3060, 2935, 2834, 1607, 1492, 1265, 1215, 1115, 1053, 986, 810, 781, 751, 701. ${ }^{1} \mathrm{H}$ NMR (400 MHz, $\left.\mathrm{CDCl}_{3}\right) \delta 7.35-6.98(\mathrm{~m}, 16 \mathrm{H}), 6.85(\mathrm{~d}, J=8.8 \mathrm{~Hz}$, $1 \mathrm{H}), 6.71(\mathrm{~d}, J=7.2 \mathrm{~Hz}, 2 \mathrm{H}), 6.59-6.52(\mathrm{~m}, 1 \mathrm{H}), 6.25(\mathrm{~d}, J=8.0 \mathrm{~Hz}, 1 \mathrm{H}), 6.20(\mathrm{dd}$, $J=8.8,2.8 \mathrm{~Hz}, 1 \mathrm{H}), 5.04(\mathrm{~d}, J=28.8 \mathrm{~Hz}, 1 \mathrm{H}), 3.43(\mathrm{~s}, 3 \mathrm{H}) .{ }^{13} \mathrm{C}$ NMR (101 MHz, $\left.\mathrm{CDCl}_{3}\right) \delta 160.1,156.5,147.5(\mathrm{~d}, J=4.9 \mathrm{~Hz}), 140.6(\mathrm{~d}, J=3.9 \mathrm{~Hz}), 134.8(\mathrm{~d}, J=2.4$ $\mathrm{Hz}), 134.2$ (d, $J=5.0 \mathrm{~Hz}), 133.4$ (d, $J=9.1 \mathrm{~Hz}), 132.1$ (d, $J=2.8 \mathrm{~Hz}), 131.7,131.4$ (d, $J=5.3 \mathrm{~Hz}), 130.8,128.4(\mathrm{~d}, J=171.6 \mathrm{~Hz}), 128.2,128.0(\mathrm{~d}, J=3.4 \mathrm{~Hz}), 127.6(\mathrm{~d}, J=$ $3.0 \mathrm{~Hz}), 127.2(\mathrm{~d}, J=6.7 \mathrm{~Hz}), 127.0,125.8,125.6,121.0,120.8,111.1,110.3$ (d, $J=$ $3.5 \mathrm{~Hz}), 62.0(\mathrm{~d}, J=2.6 \mathrm{~Hz}), 55.4,51.4(\mathrm{~d}, J=78.2 \mathrm{~Hz}) .{ }^{31} \mathrm{P} \mathrm{NMR}\left(162 \mathrm{MHz}, \mathrm{CDCl}_{3}\right)$ $\delta$ 28.8. HRMS (ESI) calcd for $\mathrm{C}_{33} \mathrm{H}_{27} \mathrm{NO}_{3} \mathrm{P}^{+}[\mathrm{M}+\mathrm{H}]^{+} m / z$ : 516.1723 ; found 516.1723.

\section{Rel-(4bS,15S,16S)-7-methyl-15,16-diphenyl-4b,16-dihydrodibenzo[b,f]benzo[4,5]}

\section{$[1,2]$ azaphosphinino $[1,6-d][1,4]$ oxazepine 15-oxide (3i)}

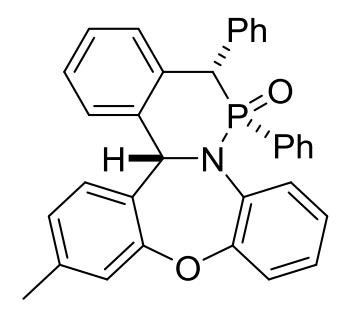

The crude residue was purified by flash chromatography (PE:EA $=4: 1$ to $1: 1, v / v)$ over silica gel. White solid $(71 \mathrm{mg}, 72 \%)$; mp. $296-298^{\circ} \mathrm{C}$. IR $(\mathrm{KBr})$ $v\left(\mathrm{~cm}^{-1}\right) 3057,2925,2859,1598,1492,1244,1208,1112,982,781,746,701,693 .{ }^{1} \mathrm{H}$ NMR (400 MHz, $\left.\mathrm{CDCl}_{3}\right) \delta 7.74(\mathrm{dd}, J=7.3,1.4 \mathrm{~Hz}, 1 \mathrm{H}), 7.30-7.01(\mathrm{~m}, 15 \mathrm{H}), 6.80-$ $6.67(\mathrm{~m}, 5 \mathrm{H}), 6.30(\mathrm{~d}, J=8.0 \mathrm{~Hz}, 1 \mathrm{H}), 5.10(\mathrm{~d}, J=28.7 \mathrm{~Hz}, 1 \mathrm{H}), 2.42(\mathrm{~s}, 3 \mathrm{H}) .{ }^{13} \mathrm{C} \mathrm{NMR}$ $\left(101 \mathrm{MHz} \mathrm{CDCl}_{3}\right) \delta 159.5,153.5(\mathrm{~d}, J=5.0 \mathrm{~Hz}), 141.4,140.8(\mathrm{~d}, J=3.8 \mathrm{~Hz}), 134.5$ (d, $J=3.4 \mathrm{~Hz}), 134.4$ (d, $J=5.2 \mathrm{~Hz}), 133.5$ (d, $J=9.0 \mathrm{~Hz}), 132.0(\mathrm{~d}, J=2.9 \mathrm{~Hz}), 131.7$ $(\mathrm{d}, J=4.2 \mathrm{~Hz}), 131.5,131.4,128.6(\mathrm{~d}, J=129.2 \mathrm{~Hz}), 128.2(\mathrm{~d}, J=2.0 \mathrm{~Hz}), 127.9(\mathrm{~d}, J$ $=2.2 \mathrm{~Hz}), 127.8,127.6(\mathrm{~d}, J=2.8 \mathrm{~Hz}), 127.3(\mathrm{~d}, J=6.7 \mathrm{~Hz}), 126.9(\mathrm{~d}, J=1.7 \mathrm{~Hz})$, 
126.4, 126.0 (d, $J=3.4 \mathrm{~Hz}), 125.9$ (d, $J=2.0 \mathrm{~Hz}), 125.3,124.9,124.5$ (d, $J=6.7 \mathrm{~Hz})$, 121.6, 120.7, $61.8(\mathrm{~d}, J=3.1 \mathrm{~Hz}), 51.5(\mathrm{~d}, J=78.6 \mathrm{~Hz}), 21.2 .{ }^{31} \mathrm{P}$ NMR (162 MHz, $\left.\mathrm{CDCl}_{3}\right) \delta$ 28.7. HRMS (ESI) calcd for $\mathrm{C}_{33} \mathrm{H}_{27} \mathrm{NO}_{2} \mathrm{P}^{+}[\mathrm{M}+\mathrm{H}]^{+} m / z$ : 500.1774; found 500.1768 .

\section{Rel-(4bS,15S,16S)-7-chloro-15,16-diphenyl-4b,16-dihydrodibenzo[b,f]benzo[4,5]}

\section{$[1,2]$ azaphosphinino $[1,6-d][1,4]$ oxazepine 15-oxide $(3 \mathrm{j})$}<smiles>O=P1(c2ccccc2)c2cc(Cl)ccc2Oc2ccccc2[C@H]1c1ccccc1</smiles>

White solid (89 mg, 86\%); mp. 302-304 ${ }^{\circ} \mathrm{C}$. IR (KBr) $v\left(\mathrm{~cm}^{-1}\right)$ 3057, 2924, 2851, 1599, 1492, 1258, 1243, 1210, 1116, 1049, 1017, 978, 784, 751, 684. ${ }^{1} \mathrm{H}$ NMR (400 MHz, $\left.\mathrm{CDCl}_{3}\right) \delta 7.79-7.72(\mathrm{~m}, 1 \mathrm{H}), 7.40(\mathrm{~d}, J=2.0 \mathrm{~Hz}, 1 \mathrm{H}), 7.30-$ $7.03(\mathrm{~m}, 14 \mathrm{H}), 6.84-6.73(\mathrm{~m}, 3 \mathrm{H}), 6.70(\mathrm{~d}, J=7.4 \mathrm{~Hz}, 2 \mathrm{H}), 6.30(\mathrm{~d}, J=7.6 \mathrm{~Hz}, 1 \mathrm{H})$, $5.08(\mathrm{~d}, J=28.1 \mathrm{~Hz}, 1 \mathrm{H}) .{ }^{13} \mathrm{C} \mathrm{NMR}\left(101 \mathrm{MHz}, \mathrm{CDCl}_{3}\right) \delta 159.4,152.9(\mathrm{~d}, J=5.1 \mathrm{~Hz})$, $139.6(\mathrm{~d}, J=4.7 \mathrm{~Hz}), 135.4,134.5,134.2(\mathrm{~d}, J=5.4 \mathrm{~Hz}), 133.5(\mathrm{~d}, J=9.0 \mathrm{~Hz}), 132.4$, 132.1, 131.9, 131.2 (d, $J=5.4 \mathrm{~Hz}), 128.31,128.29$ (d, $J=129.5 \mathrm{~Hz}), 128.1,127.9$ (d, $J=13.1 \mathrm{~Hz}), 127.6$ (d, $J=2.8 \mathrm{~Hz}), 127.1$ (d, $J=1.5 \mathrm{~Hz}), 126.6(\mathrm{~d}, J=6.5 \mathrm{~Hz}), 125.7$ $(\mathrm{d}, J=3.0 \mathrm{~Hz}), 125.4,61.9$ (d, $J=3.3 \mathrm{~Hz}), 51.5(\mathrm{~d}, J=78.9 \mathrm{~Hz}) .{ }^{31} \mathrm{P}$ NMR $(162 \mathrm{MHz}$, $\left.\mathrm{CDCl}_{3}\right) \delta$ 28.5. HRMS (ESI) calcd for $\mathrm{C}_{32} \mathrm{H}_{24} \mathrm{ClNO}_{2} \mathrm{P}^{+}[\mathrm{M}+\mathrm{H}]^{+} m / z$ : 520.1228 ; found 520.1233.

\section{Rel-(4bS,15S,16S)-6-nitro-15,16-diphenyl-4b,16-dihydrodibenzo[b, $f$ ]benzo[4,5]}

$[1,2]$ azaphosphinino[1,6-d][1,4] oxazepine 15-oxide (3k)<smiles>O=[N+]([O-])c1ccc2c(c1)C1(c3ccccc3[C@@H](c3ccccc3)P(=O)(c3ccccc3)N2c2ccccc2)c2ccccc21</smiles>

The crude residue was purified by flash chromatography (PE:EA $=5: 1$ to $1: 1$ to $1: 3, v / v$ ) over silica gel. White solid (50 mg, 47\%); mp. 303-305 ${ }^{\circ} \mathrm{C}$. IR (KBr) $v\left(\mathrm{~cm}^{-1}\right) 3059,2925,2852,1527,1492,1439,1347,1243,1114,980,750$. 
${ }^{1} \mathrm{H}$ NMR (400 MHz, $\left.\mathrm{CDCl}_{3}\right) \delta 8.30-8.20(\mathrm{~m}, 2 \mathrm{H}), 7.84-7.79(\mathrm{~m}, 1 \mathrm{H}), 7.45-7.23$ (m, 8H), $7.16-7.02(\mathrm{~m}, 4 \mathrm{H}), 6.98-6.82(\mathrm{~m}, 4 \mathrm{H}), 6.46(\mathrm{~d}, J=7.6 \mathrm{~Hz}, 2 \mathrm{H}), 6.32(\mathrm{~d}, J$ $=6.0 \mathrm{~Hz}, 1 \mathrm{H}), 5.02(\mathrm{~d}, J=25.8 \mathrm{~Hz}, 1 \mathrm{H}) .{ }^{13} \mathrm{C} \mathrm{NMR}\left(101 \mathrm{MHz}, \mathrm{CDCl}_{3}\right) \delta 161.8,152.1$ $(\mathrm{d}, J=5.5 \mathrm{~Hz}), 143.8,136.9(\mathrm{~d}, J=8.1 \mathrm{~Hz}), 135.4,133.7$ (d, $J=9.0 \mathrm{~Hz}), 133.5(\mathrm{~d}, J=$ $6.4 \mathrm{~Hz}), 133.1$ (d, $J=3.9 \mathrm{~Hz}), 132.3$ (d, $J=1.9 \mathrm{~Hz}), 131.0(\mathrm{~d}, J=8.0 \mathrm{~Hz}), 130.3$ (d, $J$ $=4.9 \mathrm{~Hz}), 129.9(\mathrm{~d}, J=5.2 \mathrm{~Hz}), 129.3,128.3,128.0,128.0(\mathrm{~d}, J=130.7 \mathrm{~Hz}), 127.9$, 127.8, 127.6, 127.2 (d, $J=3.3 \mathrm{~Hz}), 127.2$ (d, $J=2.1 \mathrm{~Hz}), 126.7,126.2,125.4,122.3$, 120.8, $63.7(\mathrm{~d}, J=1.8 \mathrm{~Hz}), 51.5(\mathrm{~d}, J=80.7 \mathrm{~Hz}) .{ }^{31} \mathrm{P} \mathrm{NMR}\left(162 \mathrm{MHz}, \mathrm{CDCl}_{3}\right) \delta 28.5$. HRMS (ESI) calcd for $\mathrm{C}_{32} \mathrm{H}_{24} \mathrm{~N}_{2} \mathrm{O}_{4} \mathrm{P}^{+}[\mathrm{M}+\mathrm{H}]^{+} \mathrm{m} / z$ : 531.1468; found 531.1458.

\section{Rel-(4bS,15S,16S)-11-nitro-15,16-diphenyl-4b,16-dihydrodibenzo[b,f]benzo[4,5]}

\section{$[1,2]$ azaphosphinino $[1,6-d][1,4]$ oxazepine 15-oxide (3l)}

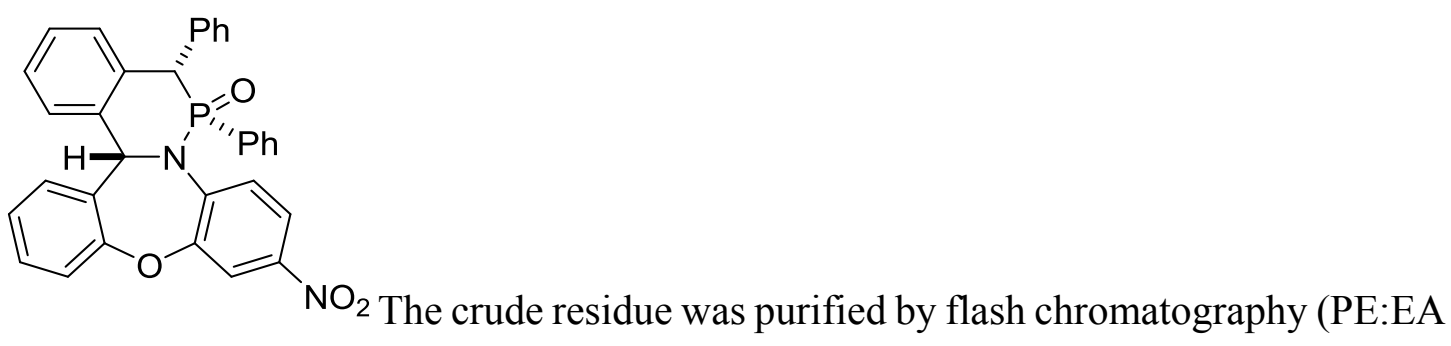
$=4: 1$ to $1: 1, v / v)$ over silica gel. Yellow solid $(25 \mathrm{mg}, 24 \%) ; \mathrm{mp} .300-302{ }^{\circ} \mathrm{C}$. IR $(\mathrm{KBr})$ $v\left(\mathrm{~cm}^{-1}\right) 3060,2925,2854,1736,1590,1517,1491,1341,1259,1243,1211,974,782$, 745, 700. ${ }^{1} \mathrm{H} \mathrm{NMR}\left(400 \mathrm{MHz}, \mathrm{CDCl}_{3}\right) \delta 7.85(\mathrm{~d}, J=2.5 \mathrm{~Hz}, 1 \mathrm{H}), 7.78(\mathrm{~d}, J=9.0 \mathrm{~Hz}$, 1H), $7.54-7.33(\mathrm{~m}, 4 \mathrm{H}), 7.30-6.97(\mathrm{~m}, 12 \mathrm{H}), 6.75(\mathrm{~d}, J=6.6 \mathrm{~Hz}, 2 \mathrm{H}), 6.61-6.53$ $(\mathrm{m}, 1 \mathrm{H}), 6.35(\mathrm{~d}, J=9.1 \mathrm{~Hz}, 1 \mathrm{H}), 5.15(\mathrm{~d}, J=29.8 \mathrm{~Hz}, 1 \mathrm{H}) .{ }^{13} \mathrm{C}$ NMR $(101 \mathrm{MHz}$, $\left.\mathrm{CDCl}_{3}\right) \delta 159.1,152.0(\mathrm{~d}, J=4.9 \mathrm{~Hz}), 143.1,141.6,140.0(\mathrm{~d}, J=2.9 \mathrm{~Hz}), 134.0(\mathrm{~d}, J$ $=5.0 \mathrm{~Hz}), 132.7(\mathrm{~d}, J=3.0 \mathrm{~Hz}), 132.0,131.7,131.6(\mathrm{~d}, J=5.6 \mathrm{~Hz}), 130.5(\mathrm{~d}, J=4.2$ $\mathrm{Hz}), 128.5(\mathrm{~d}, J=2.3 \mathrm{~Hz}), 128.4(\mathrm{~d}, J=1.3 \mathrm{~Hz}), 128.1(\mathrm{~d}, J=3.0 \mathrm{~Hz}), 127.8$ (d, $J=$ $126.3 \mathrm{~Hz}), 127.3,126.9$ (d, $J=6.8 \mathrm{~Hz}), 126.8,126.7$ (d, $J=6.6 \mathrm{~Hz}), 125.3$ (d, $J=2.3$ Hz), 125.1 (d, $J=3.7 \mathrm{~Hz}), 121.0,120.8,116.8,61.9$ (d, $J=3.0 \mathrm{~Hz}), 51.4$ (d, $J=78.3$ Hz). ${ }^{31} \mathrm{P} \mathrm{NMR}\left(162 \mathrm{MHz}, \mathrm{CDCl}_{3}\right) \delta 30.3$. HRMS (ESI) calcd for $\mathrm{C}_{32} \mathrm{H}_{24} \mathrm{~N}_{2} \mathrm{O}_{4} \mathrm{P}^{+}[\mathrm{M}+\mathrm{H}]^{+}$ $\mathrm{m} / \mathrm{z}$ : 531.1468; found 531.1467 . 


\section{Rel-(4bS,15S,16S)-7,11-dichloro-15,16-diphenyl-4b,16-dihydrodibenzo[ $b, f]$ benzo}

$[4,5][1,2]$ azaphosphinino[1,6-d][1,4]oxazepine 15-oxide $(3 \mathrm{~m})$<smiles></smiles>

The crude residue was purified by flash chromatography (PE:EA $=4: 1$ to $1: 1, v / v)$ over silica gel. White solid (108 mg, 97\%); mp. 288-290 ${ }^{\circ} \mathrm{C}$. IR $(\mathrm{KBr}) v\left(\mathrm{~cm}^{-1}\right) 3059,2924,2852,1597,1492,1261,1243,1210,1116,1049,1017$, 976, 783, 750, 689. ${ }^{1} \mathrm{H}$ NMR $\left(400 \mathrm{MHz}, \mathrm{CDCl}_{3}\right) \delta 7.73(\mathrm{~d}, J=8.8 \mathrm{~Hz}, 1 \mathrm{H}), 7.40(\mathrm{~d}$, $J=2.0 \mathrm{~Hz}, 1 \mathrm{H}), 7.31-7.19(\mathrm{~m}, 6 \mathrm{H}), 7.16-7.03(\mathrm{~m}, 8 \mathrm{H}), 6.73(\mathrm{~m}, 4 \mathrm{H}), 6.30(\mathrm{~d}, J=$ $7.6 \mathrm{~Hz}, 1 \mathrm{H}), 5.09$ (d, $J=28.4 \mathrm{~Hz}, 1 \mathrm{H}) .{ }^{13} \mathrm{C} \mathrm{NMR}\left(101 \mathrm{MHz}, \mathrm{CDCl}_{3}\right) \delta 159.1,153.1(\mathrm{~d}$, $J=4.9 \mathrm{~Hz}), 139.5(\mathrm{~d}, J=4.0 \mathrm{~Hz}), 135.7,134.1(\mathrm{~d}, J=5.3 \mathrm{~Hz}), 133.4(\mathrm{~d}, J=9.1 \mathrm{~Hz})$, 132.5, 132.3 (d, $J=1.7 \mathrm{~Hz}), 131.5(\mathrm{~d}, J=3.9 \mathrm{~Hz}), 131.2(\mathrm{~d}, J=5.3 \mathrm{~Hz}), 128.77$ (d, $J=$ $166.0 \mathrm{~Hz}), 128.75(\mathrm{~d}, J=9.8 \mathrm{~Hz}), 128.4,128.2,128.1(\mathrm{~d}, J=13.5 \mathrm{~Hz}), 127.7$ (d, $J=$ $2.0 \mathrm{~Hz}), 127.1,126.9$ (d, $J=2.7 \mathrm{~Hz}), 126.3$ (d, $J=6.4 \mathrm{~Hz}), 126.1,126.0,125.7,121.7$, 121.2, $61.7(\mathrm{~d}, J=1.4 \mathrm{~Hz}), 51.4(\mathrm{~d}, J=78.7 \mathrm{~Hz}) .{ }^{31} \mathrm{P} \mathrm{NMR}\left(162 \mathrm{MHz}, \mathrm{CDCl}_{3}\right) \delta 28.9$. HRMS (ESI) calcd for $\mathrm{C}_{32} \mathrm{H}_{23} \mathrm{Cl}_{2} \mathrm{NO}_{2} \mathrm{P}^{+}[\mathrm{M}+\mathrm{H}]^{+} \mathrm{m} / z$ : 554.0838; found 554.0841.

\section{Rel-(4bS,15S,16S)-7,11-dimethyl-15,16-diphenyl-4b,16-dihydrodibenzo $[b, f]$ benzo} $[4,5][1,2]$ azaphosphinino[1,6- $d][1,4]$ oxazepine 15-oxide (3n)

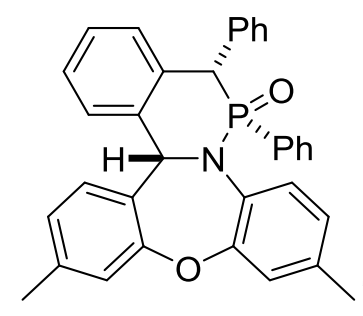

The crude residue was purified by flash chromatography (PE:EA $=4: 1$ to $1: 1, v / v)$ over silica gel. White solid $(63 \mathrm{mg}, 78 \%)$; mp. $290-292{ }^{\circ} \mathrm{C}$. IR $(\mathrm{KBr})$ $v\left(\mathrm{~cm}^{-1}\right) 3060,2923,2854,1502,1258,1211,1127,1088,944,782,730,544 .{ }^{1} \mathrm{H}$ NMR $\left(400 \mathrm{MHz}, \mathrm{CDCl}_{3}\right) \delta 7.63(\mathrm{~d}, J=8.2 \mathrm{~Hz}, 1 \mathrm{H}), 7.31-7.00(\mathrm{~m}, 15 \mathrm{H}), 6.84(\mathrm{~d}, J=2.0$ $\mathrm{Hz}, 1 \mathrm{H}), 6.74(\mathrm{~d}, J=7.4 \mathrm{~Hz}, 2 \mathrm{H}), 6.70-6.66(\mathrm{~m}, 1 \mathrm{H}), 6.27$ (d, $J=7.8 \mathrm{~Hz}, 1 \mathrm{H}), 5.08$ $(\mathrm{d}, J=28.4 \mathrm{~Hz}, 1 \mathrm{H}), 2.41(\mathrm{~s}, 3 \mathrm{H}), 2.06(\mathrm{~s}, 3 \mathrm{H}) .{ }^{13} \mathrm{C} \mathrm{NMR}\left(101 \mathrm{MHz}, \mathrm{CDCl}_{3}\right) \delta 159.4$, $153.3(\mathrm{~d}, J=5.0 \mathrm{~Hz}), 141.1,140.8(\mathrm{~d}, J=4.1 \mathrm{~Hz}), 135.0,134.3(\mathrm{~d}, J=5.3 \mathrm{~Hz}), 133.6$ 
(d, $J=9.1 \mathrm{~Hz}), 131.9(\mathrm{~d}, J=6.8 \mathrm{~Hz}), 131.9,131.7(\mathrm{~d}, J=3.2 \mathrm{~Hz}), 131.40,131.35$ (d, $J$ $=5.6 \mathrm{~Hz}), 128.8,128.7(\mathrm{~d}, J=129.5 \mathrm{~Hz}), 128.4,128.1(\mathrm{~d}, J=2.3 \mathrm{~Hz}), 127.9(\mathrm{~d}, J=4.5$ Hz), 127.8, 127.5 (d, $J=2.9 \mathrm{~Hz}), 127.4(\mathrm{~d}, J=6.9 \mathrm{~Hz}), 126.9$ (d, $J=1.8 \mathrm{~Hz}), 126.2$, $126.0(\mathrm{~d}, J=2.3 \mathrm{~Hz}), 125.84,125.81,124.6(\mathrm{~d}, J=6.6 \mathrm{~Hz}), 121.6,121.2,61.9(\mathrm{~d}, J=$ $3.4 \mathrm{~Hz}), 51.5(\mathrm{~d}, J=78.5 \mathrm{~Hz}), 21.2,20.5 .{ }^{31} \mathrm{P} \mathrm{NMR}\left(162 \mathrm{MHz}, \mathrm{CDCl}_{3}\right) \delta 28.3$. HRMS (ESI) calcd for $\mathrm{C}_{34} \mathrm{H}_{29} \mathrm{NO}_{2} \mathrm{P}^{+}[\mathrm{M}+\mathrm{H}]^{+} m / z$ : 514.1930; found 514.1935.

\section{Rel-(4bS,15S,16S)-15,16-diphenyl-4b,16-dihydrodibenzo[b,f]benzo[4,5][1,2]aza-} phosphinino[1,6- $d][1,4]$ thiazepine 15-oxide (3o)<smiles></smiles>

White solid (75 mg, 75\%); mp. 296-298 ${ }^{\circ} \mathrm{C}$. IR (KBr) $v\left(\mathrm{~cm}^{-1}\right) 3060$, 2971, 2927, 1736, 1618, 1471, 1438, 1324, 1220, 1066, 975, 750, 701, 534. ${ }^{1} \mathrm{H}$ NMR $\left(400 \mathrm{MHz}, \mathrm{CDCl}_{3}\right) \delta 8.01(\mathrm{~d}, J=8.0 \mathrm{~Hz}, 1 \mathrm{H}), 7.67-7.62(\mathrm{~m}, 1 \mathrm{H}), 7.32-6.97(\mathrm{~m}$, $15 \mathrm{H}), 6.89(\mathrm{td}, J=7.8,1.6 \mathrm{~Hz}, 1 \mathrm{H}), 6.81-6.71(\mathrm{~m}, 2 \mathrm{H}), 6.66(\mathrm{td}, J=7.6,1.6 \mathrm{~Hz}, 1 \mathrm{H})$, $6.42-6.27(\mathrm{~m}, 2 \mathrm{H}), 5.04(\mathrm{~d}, J=29.6 \mathrm{~Hz}, 1 \mathrm{H}) .{ }^{13} \mathrm{C} \mathrm{NMR}\left(101 \mathrm{MHz}, \mathrm{CDCl}_{3}\right) \delta 141.9$ $(\mathrm{d}, J=3.7 \mathrm{~Hz}), 140.8(\mathrm{~d}, J=2.2 \mathrm{~Hz}), 140.4,137.0$ (d, $J=6.8 \mathrm{~Hz}), 135.7$ (d, $J=5.2 \mathrm{~Hz})$, $135.0(\mathrm{~d}, J=4.8 \mathrm{~Hz}), 133.5,133.4,133.2,133.0,131.9$ (d, $J=2.9 \mathrm{~Hz}), 131.7$ (d, $J=$ $5.6 \mathrm{~Hz}), 131.6$ (d, $J=4.4 \mathrm{~Hz}), 129.5$ (d, $J=7.0 \mathrm{~Hz}), 129.3,128.9$ (d, $J=3.1 \mathrm{~Hz}), 128.2$ $(\mathrm{d}, J=2.0 \mathrm{~Hz}), 127.9,127.7,127.6,127.6,126.5(\mathrm{~d}, J=6.7 \mathrm{~Hz}), 126.5(\mathrm{~d}, J=2.3$ $\mathrm{Hz}), 126.3(\mathrm{~d}, J=1.8 \mathrm{~Hz}), 125.1,63.5$ (d, $J=3.7 \mathrm{~Hz}), 51.6(\mathrm{~d}, J=77.8 \mathrm{~Hz}) .{ }^{31} \mathrm{P}$ NMR $\left(162 \mathrm{MHz} \mathrm{CDCl}_{3}\right) \delta$ 29.5. HRMS (ESI) calcd for $\mathrm{C}_{32} \mathrm{H}_{25} \mathrm{NOPS}^{+}[\mathrm{M}+\mathrm{H}]^{+} m / z: 502.1389$; found 502.1389 .

Rel-(4bS,15S,16S)-7-methyl-15,16-diphenyl-4b,16-dihydrodibenzo[b, $f]$ benzo[4,5] $[1,2]$ azaphosphinino $[1,6-d][1,4]$ thiazepine 15-oxide (3p) 


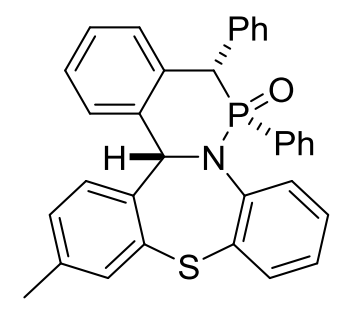

White solid (71 mg, 69\%); mp. $306-308{ }^{\circ} \mathrm{C}$. IR $(\mathrm{KBr}) v\left(\mathrm{~cm}^{-1}\right)$ 3059, 2974, 1599, 1470, 1438, 1218, 1064, 975, 779, 700, 530. ${ }^{1} \mathrm{H}$ NMR (400 MHz, $\left.\mathrm{CDCl}_{3}\right) \delta 8.00(\mathrm{~d}, J=8.0 \mathrm{~Hz}, 1 \mathrm{H}), 7.47(\mathrm{~d}, J=2.0 \mathrm{~Hz}, 1 \mathrm{H}), 7.27-6.97(\mathrm{~m}, 14 \mathrm{H}), 6.89$ $(\operatorname{td}, J=8.0,1.6 \mathrm{~Hz}, 1 \mathrm{H}), 6.77(\mathrm{dd}, J=6.8,2.0 \mathrm{~Hz}, 2 \mathrm{H}), 6.66(\mathrm{td}, J=7.6,1.2 \mathrm{~Hz}, 1 \mathrm{H})$, $6.41(\mathrm{dd}, J=7.2,1.6 \mathrm{~Hz}, 1 \mathrm{H}), 6.30(\mathrm{~d}, J=10.0 \mathrm{~Hz}, 1 \mathrm{H}), 5.03(\mathrm{~d}, J=29.6 \mathrm{~Hz}, 1 \mathrm{H}) .{ }^{13} \mathrm{C}$ NMR (101 MHz, $\left.\mathrm{CDCl}_{3}\right) \delta 142.0(\mathrm{~d}, J=3.7 \mathrm{~Hz}), 141.1(\mathrm{~d}, J=2.2 \mathrm{~Hz}), 140.0,139.7$, $135.8(\mathrm{~d}, J=5.3 \mathrm{~Hz}), 135.1(\mathrm{~d}, J=4.9 \mathrm{~Hz}), 134.3,133.9(\mathrm{~d}, J=6.9 \mathrm{~Hz}), 133.5(\mathrm{~d}, J=$ $9.6 \mathrm{~Hz}), 133.1(\mathrm{~d}, J=4.1 \mathrm{~Hz}), 131.9(\mathrm{~d}, J=2.9 \mathrm{~Hz}), 131.8(\mathrm{~d}, J=5.5 \mathrm{~Hz}), 131.7,130.3$, 129.3, $129.0(\mathrm{~d}, J=131.5 \mathrm{~Hz}), 129.0(\mathrm{~d}, J=3.0 \mathrm{~Hz}), 128.3(\mathrm{~d}, J=2.1 \mathrm{~Hz}), 127.9(\mathrm{~d}, J$ $=13.2 \mathrm{~Hz}), 127.64(\mathrm{~d}, J=2.7 \mathrm{~Hz}), 127.57,126.6,126.5,126.3(\mathrm{~d}, J=1.8 \mathrm{~Hz}), 125.0$, $63.3(\mathrm{~d}, J=3.6 \mathrm{~Hz}), 51.7(\mathrm{~d}, J=77.8 \mathrm{~Hz}), 21.1 .{ }^{31} \mathrm{P} \mathrm{NMR}\left(162 \mathrm{MHz}, \mathrm{CDCl}_{3}\right) \delta 29.5$. HRMS (ESI) calcd for $\mathrm{C}_{33} \mathrm{H}_{27} \mathrm{NOPS}^{+}[\mathrm{M}+\mathrm{H}]^{+} \mathrm{m} / z$ : 516.1545; found 516.1540 .

\section{Rel-(4bS,15S,16S)-8-fluoro-15,16-diphenyl-4b,16-dihydrodibenzo[b, $f]$ benzo[4,5]}

\section{$[1,2]$ azaphosphinino[1,6- $d][1,4]$ thiazepine 15-oxide (3q)}

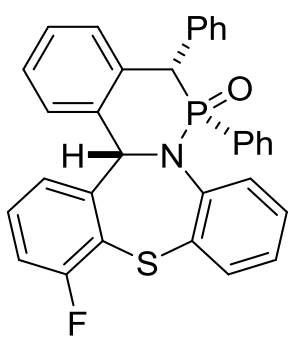

White solid (75 mg, 72\%); mp. 290-292 ${ }^{\circ} \mathrm{C}$. IR (KBr) $v\left(\mathrm{~cm}^{-1}\right) 3060$, 2925, 2849, 1578, 1474, 1459, 1528, 1215, 1114, 1069, 1049, 986, 950, 781, 751, 702, 532. ${ }^{1} \mathrm{H}$ NMR (400 MHz, CDCl$) \delta 8.01(\mathrm{~d}, J=8.0 \mathrm{~Hz}, 1 \mathrm{H}), 7.33-7.00(\mathrm{~m}, 16 \mathrm{H}), 6.77$ (d, $J=7.6 \mathrm{~Hz}, 2 \mathrm{H}), 6.74-6.65(\mathrm{~m}, 1 \mathrm{H}), 6.45(\mathrm{~d}, J=6.8 \mathrm{~Hz}, 1 \mathrm{H}), 6.38(\mathrm{~d}, J=10.0 \mathrm{~Hz}$, $1 \mathrm{H}), 5.04(\mathrm{~d}, J=29.6 \mathrm{~Hz}, 1 \mathrm{H}) .{ }^{13} \mathrm{C} \mathrm{NMR}\left(101 \mathrm{MHz}, \mathrm{CDCl}_{3}\right) \delta 161.8\left(\mathrm{~d}, J_{\mathrm{C}-\mathrm{F}}=246.3\right.$ $\mathrm{Hz}), 142.3(\mathrm{~d}, J=2.6 \mathrm{~Hz}), 140.3,139.7(\mathrm{~d}, J=6.6 \mathrm{~Hz}), 135.0(\mathrm{~d}, J=4.8 \mathrm{~Hz}), 134.7$ (d, $J=5.2 \mathrm{~Hz}), 133.6,133.4(\mathrm{~d}, J=9.5 \mathrm{~Hz}), 132.1(\mathrm{~d}, J=2.1 \mathrm{~Hz}), 131.7(\mathrm{~d}, J=5.7 \mathrm{~Hz})$, $131.5(\mathrm{~d}, J=4.0 \mathrm{~Hz}), 130.6(\mathrm{~d}, J=8.3 \mathrm{~Hz}), 129.8,129.2(\mathrm{~d}, J=2.3 \mathrm{~Hz}), 128.7\left(\mathrm{~d}, J_{\mathrm{C}-}\right.$ 
$\mathrm{P}=132.7 \mathrm{~Hz}), 128.6(\mathrm{~d}, J=2.5 \mathrm{~Hz}), 128.3,128.0,127.9(\mathrm{~d}, J=6.5 \mathrm{~Hz}), 127.7(\mathrm{~d}, J=$ $1.7 \mathrm{~Hz}), 126.8$ (d, $J=6.6 \mathrm{~Hz}), 126.4(\mathrm{~d}, J=10.3 \mathrm{~Hz}), 125.3,116.94(\mathrm{~d}, J=23.9 \mathrm{~Hz})$, $63.1\left(\mathrm{dd}, J_{\mathrm{C}-\mathrm{F}, \mathrm{C}-\mathrm{P}}=2.5,2.5 \mathrm{~Hz}\right), 51.6(\mathrm{~d}, J=77.9 \mathrm{~Hz}) .{ }^{19} \mathrm{~F} \mathrm{NMR}\left(376 \mathrm{MHz}, \mathrm{CDCl}_{3}\right) \delta-$ 109.35. ${ }^{31} \mathrm{P}$ NMR $\left(162 \mathrm{MHz}, \mathrm{CDCl}_{3}\right) \delta$ 29.8. HRMS (ESI) calcd for $\mathrm{C}_{32} \mathrm{H}_{24} \mathrm{FNOPS}^{+}$ $[\mathrm{M}+\mathrm{H}]^{+} m / z: 520.1295$; found 520.1299.

Rel-(4bS,15S,16S)-12-chloro-15,16-diphenyl-4b,16-dihydrodibenzo[b,f]benzo[4,5] $[1,2]$ azaphosphinino[1,6- $d][1,4]$ thiazepine 15-oxide (3r)

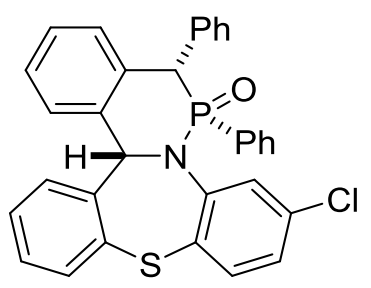

White solid (93 mg, 89\%); mp. 286-288 ${ }^{\circ} \mathrm{C}$. IR (KBr) $v\left(\mathrm{~cm}^{-1}\right)$ $3059,2925,2849,1736,1572,1460,1439,1214,1115,1014,985,781,750,697,538$, 521. ${ }^{1} \mathrm{H}$ NMR (400 MHz, $\left.\mathrm{CDCl}_{3}\right) \delta 8.17(\mathrm{~s}, 1 \mathrm{H}), 7.74(\mathrm{~d}, J=6.8 \mathrm{~Hz}, 1 \mathrm{H}), 7.45-7.12$ $(\mathrm{m}, 15 \mathrm{H}), 6.90(\mathrm{~d}, J=6.2 \mathrm{~Hz}, 2 \mathrm{H}), 6.76(\mathrm{~d}, J=7.5 \mathrm{~Hz}, 1 \mathrm{H}), 6.45(\mathrm{dd}, J=23.6,8.3 \mathrm{~Hz}$, $2 \mathrm{H}), 5.15(\mathrm{~d}, J=29.8 \mathrm{~Hz}, 1 \mathrm{H}) .{ }^{13} \mathrm{C} \mathrm{NMR}\left(101 \mathrm{MHz}, \mathrm{CDCl}_{3}\right) \delta 143.0(\mathrm{~d}, J=2.9 \mathrm{~Hz})$, 140.5, 139.8, 136.7 (d, $J=6.8 \mathrm{~Hz}), 134.8,134.8$ (d, $J=5.1 \mathrm{~Hz}), 134.2(\mathrm{~d}, J=5.2 \mathrm{~Hz})$, $133.5(\mathrm{~d}, J=4.5 \mathrm{~Hz}), 133.4,133.3,132.1(\mathrm{~d}, J=2.7 \mathrm{~Hz}), 131.7$ (d, $J=5.8 \mathrm{~Hz}), 131.2$, $129.8(\mathrm{~d}, J=10.1 \mathrm{~Hz}), 129.0(\mathrm{~d}, J=2.7 \mathrm{~Hz}), 128.6(\mathrm{~d}, J=131.4 \mathrm{~Hz}), 128.3(\mathrm{~d}, J=2.1$ Hz), $128.0(\mathrm{~d}, J=13.1 \mathrm{~Hz}), 127.7,126.6(\mathrm{~d}, J=6.8 \mathrm{~Hz}), 126.4,125.1,63.6(\mathrm{~d}, J=3.5$ $\mathrm{Hz}), 51.6(\mathrm{~d}, J=77.5 \mathrm{~Hz}) .{ }^{31} \mathrm{P}$ NMR $\left(162 \mathrm{MHz}, \mathrm{CDCl}_{3}\right) \delta 29.8$. HRMS (ESI) calcd for $\mathrm{C}_{32} \mathrm{H}_{24} \mathrm{ClNOPS}^{+}[\mathrm{M}+\mathrm{H}]^{+} m / z$ : 536.0999; found 536.1003.

Rel-(4bS,15S,16R)-16-(2-fluorophenyl)-11-methyl-15-phenyl-4b,16-dihydrodibenzo $[b, f]$ benzo $[4,5][1,2]$ azaphosphinino[1,6- $d][1,4]$ oxazepine 15-oxide (3s)<smiles></smiles>

White solid (90 mg, 87\%); mp. 272-274 ${ }^{\circ} \mathrm{C} .{ }^{1} \mathrm{H}$ NMR (400 MHz, $\left.\mathrm{CDCl}_{3}\right) \delta 7.57(\mathrm{~d}, J=2.0 \mathrm{~Hz}, 1 \mathrm{H}), 7.43-7.18(\mathrm{~m}, 10 \mathrm{H}), 7.12-7.06(\mathrm{~m}, 3 \mathrm{H}), 7.02(\mathrm{t}$, 
$J=9.2 \mathrm{~Hz}, 1 \mathrm{H}), 6.91(\mathrm{~d}, J=8.0 \mathrm{~Hz}, 1 \mathrm{H}), 6.79(\mathrm{dd}, J=7.2,1.6 \mathrm{~Hz}, 1 \mathrm{H}), 6.68(\mathrm{t}, J=7.6$ $\mathrm{Hz}, 1 \mathrm{H}), 6.59$ (dd, $J=8.0,2.0 \mathrm{~Hz}, 1 \mathrm{H}), 6.32(\mathrm{~d}, J=7.2 \mathrm{~Hz}, 1 \mathrm{H}), 6.20(\mathrm{tt}, J=7.6,2.0$ $\mathrm{Hz}, 1 \mathrm{H}), 5.53(\mathrm{~d}, J=27.8 \mathrm{~Hz}, 1 \mathrm{H}), 2.01(\mathrm{~s}, 3 \mathrm{H}) .{ }^{31} \mathrm{P} \mathrm{NMR}\left(162 \mathrm{MHz}, \mathrm{CDCl}_{3}\right) \delta 27.28$ $(\mathrm{d}, J=1.2 \mathrm{~Hz}) .{ }^{13} \mathrm{C} \mathrm{NMR}\left(101 \mathrm{MHz}, \mathrm{CDCl}_{3}\right) \delta 161.6\left(\mathrm{dd}, J_{\mathrm{C}-\mathrm{F}, \mathrm{C}-\mathrm{P}}=247.7,6.3 \mathrm{~Hz}\right)$, 159.1, $151.4(\mathrm{~d}, J=5.3 \mathrm{~Hz}), 140.1(\mathrm{~d}, J=4.6 \mathrm{~Hz}), 135.1,134.2(\mathrm{~d}, J=2.7 \mathrm{~Hz}), 133.4$ $(\mathrm{d}, J=9.2 \mathrm{~Hz}), 133.3$ (d, $J=5.7 \mathrm{~Hz}), 132.0(\mathrm{~d}, J=2.2 \mathrm{~Hz}), 131.4,130.5,129.1$ (d, $J=$ $8.3 \mathrm{~Hz}), 128.6\left(\mathrm{~d}, J_{\mathrm{C}-\mathrm{P}}=134.0 \mathrm{~Hz}\right), 128.2,128.0,127.8(\mathrm{~d}, J=13.5 \mathrm{~Hz}), 127.0,126.9$, 125.9, 125.3, 123.1, 121.1, 120.2, 120.1 (d, $J=3.6 \mathrm{~Hz}), 120.0$ (d, $J=3.3 \mathrm{~Hz}), 115.7$ (d, $J=22.9 \mathrm{~Hz}), 62.7(\mathrm{~d}, J=3.1 \mathrm{~Hz}), 42.5(\mathrm{~d}, J=81.1 \mathrm{~Hz}), 20.7 .{ }^{31} \mathrm{P}$ NMR $(162 \mathrm{MHz}$, $\left.\mathrm{CDCl}_{3}\right) \delta 27.3(\mathrm{~d}, J=1.6 \mathrm{~Hz}) .{ }^{19} \mathrm{~F}$ NMR $\left(377 \mathrm{MHz}, \mathrm{CDCl}_{3}\right) \delta$-114.22. HRMS (ESI) calcd for $\mathrm{C}_{33} \mathrm{H}_{26} \mathrm{FNO}_{2} \mathrm{P}^{+}[\mathrm{M}+\mathrm{H}]^{+} m / z$ : 518.1680; found 518.1677.

\section{Rel-(4bS,15S,16R)-11-chloro-16-(2-fluorophenyl)-15-phenyl-4b,16-dihydro-} dibenzo $[b, f]$ benzo $[4,5][1,2]$ azaphosphinino $[1,6-d][1,4]$ oxazepine 15-oxide (3t)<smiles></smiles>

White solid (70 mg, 65\%); mp. 280-282 ${ }^{\circ} \mathrm{C}$. IR (KBr) $v\left(\mathrm{~cm}^{-1}\right)$ $3061,1736,1585,1488,1237,1214,1113,902,783,749,695,576 .{ }^{1} \mathrm{H}$ NMR (400 $\left.\mathrm{MHz}, \mathrm{CDCl}_{3}\right) \delta 7.67-7.63(\mathrm{~m}, 1 \mathrm{H}), 7.39-6.97(\mathrm{~m}, 15 \mathrm{H}), 6.70-6.58(\mathrm{~m}, 3 \mathrm{H}), 6.27$ $(\mathrm{d}, J=7.4 \mathrm{~Hz}, 1 \mathrm{H}), 6.11(\mathrm{t}, J=7.5 \mathrm{~Hz}, 1 \mathrm{H}), 5.50(\mathrm{~d}, J=28.2 \mathrm{~Hz}, 1 \mathrm{H}) .{ }^{13} \mathrm{C}$ NMR $(101$ $\left.\mathrm{MHz}, \mathrm{CDCl}_{3}\right) \delta 161.6\left(\mathrm{dd}, J_{\mathrm{C}-\mathrm{F}, \mathrm{C}-\mathrm{P}}=247.8,6.6 \mathrm{~Hz}\right), 158.82,153.77(\mathrm{~d}, J=5.1 \mathrm{~Hz})$, 140.1 (d, $J=4.4 \mathrm{~Hz}), 133.4$ (d, $J=8.9 \mathrm{~Hz}), 133.2$ (d, $J=5.4 \mathrm{~Hz}), 132.4$ (d, $J=2.8 \mathrm{~Hz})$, 131.6, $131.5(\mathrm{dd}, J=4.4,2.4 \mathrm{~Hz}), 130.9,129.6,129.3$ (dd, $J=8.5,2.6 \mathrm{~Hz}), 128.3,128.2$ $\left(\mathrm{d}, J_{\mathrm{C}-\mathrm{P}}=133.4 \mathrm{~Hz}\right), 128.1(\mathrm{~d}, J=13.1 \mathrm{~Hz}), 127.64,127.56,127.2(\mathrm{~d}, J=1.6 \mathrm{~Hz}), 127.0$ (d, $J=3.3 \mathrm{~Hz}), 126.4$ (d, $J=1.9 \mathrm{~Hz}), 125.9,125.4,123.2$ (dd, $J=3.0,3.0 \mathrm{~Hz}), 121.2$ (d, $J=19.6 \mathrm{~Hz}), 119.4(\mathrm{dd}, J=13.8,3.8 \mathrm{~Hz}), 116.0(\mathrm{~d}, J=1.9 \mathrm{~Hz}), 115.8$ (d, $J=1.8$ $\mathrm{Hz}), 62.4(\mathrm{~d}, J=2.9 \mathrm{~Hz}), 42.2(\mathrm{~d}, J=79.0 \mathrm{~Hz}) .{ }^{31} \mathrm{P} \mathrm{NMR}\left(162 \mathrm{MHz}, \mathrm{CDCl}_{3}\right) \delta 28.0(\mathrm{~d}$, 
$J=2.9 \mathrm{~Hz}) .{ }^{19} \mathrm{~F}$ NMR $\left(376 \mathrm{MHz}, \mathrm{CDCl}_{3}\right) \delta$-114.19. RMS (ESI) calcd for $\mathrm{C}_{32} \mathrm{H}_{23} \mathrm{ClFNO}_{2} \mathrm{P}^{+}[\mathrm{M}+\mathrm{H}]^{+} m / z$ : 538. 1133; found 538.1128.

Rel-(4bS,15S,16S)-11-methyl-16-(4-methylphenyl)-15-phenyl-4b,16-dihydrodibenzo $[b, f]$ benzo $[4,5][1,2]$ azaphosphinino[1,6- $d][1,4]$ oxazepine 15 -oxide $(3 \mathrm{u})$ and rel-(4bS,15S,16S)-3,11-dimethyl-15,16-diphenyl-4b,16-dihydrodibenzo[ $b, f]$ benzo $[4,5][1,2]$ azaphosphinino[1,6- $d][1,4]$ oxazepine 15-oxide $\left(3 u^{\prime}\right)$

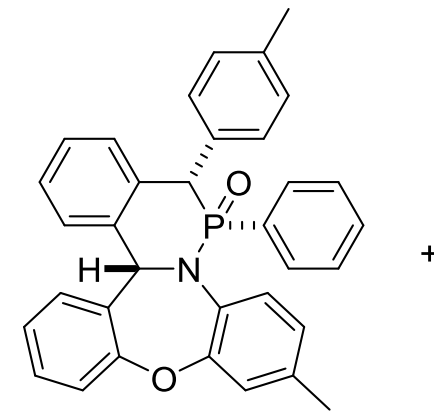

$3 \mathbf{u}$

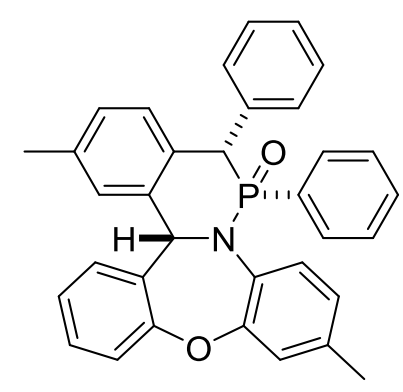

$3 u^{\prime}$

White solid (90 mg, 85\%); mp. 292-294 ${ }^{\circ} \mathrm{C}$. IR (KBr) $v\left(\mathrm{~cm}^{-1}\right)$ 3058, 3028, 2922, 2855, 1600, 1504, 1490, 1264, 1213, 1119, 989, 908, 825, 731, 696, 571, 523. ${ }^{1} \mathrm{H}$ NMR (400 MHz, $\left.\mathrm{CDCl}_{3}\right) \delta 7.56(\mathrm{~d}, J=2.0$ $\mathrm{Hz}, 1 \mathrm{H}), 7.40-7.02(\mathrm{~m}, 13 \mathrm{H}), 6.95-6.89(\mathrm{~m}, 2 \mathrm{H}), 6.74-6.61(\mathrm{~m}, 2 \mathrm{H}), 6.60-6.46$ (m, 2H), $6.33-6.20(\mathrm{~m}, 1 \mathrm{H}), 5.07(\mathrm{~d}, J=28.7 \mathrm{~Hz}, 0.36 \times 1 \mathrm{H}, 3 \mathbf{u}), 5.01(\mathrm{~d}, J=27.9$ $\left.\mathrm{Hz}, 0.64 \times 1 \mathrm{H}, 3 \mathbf{u}^{\prime}\right), 2.28$ (s, 3H), 1.99 (s, $\left.0.64 \times 3 \mathrm{H}, 3 \mathbf{u}^{\prime}\right), 1.97(\mathrm{~s}, 0.36 \times 3 \mathrm{H}, 3 \mathbf{u}) .{ }^{13} \mathrm{C}$ NMR (101 MHz, $\left.\mathrm{CDCl}_{3}\right) \delta 159.7,159.2,151.20$ (d, $\left.J=4.2 \mathrm{~Hz}\right), 151.15$ (d, $\left.J=5.1 \mathrm{~Hz}\right)$, $140.5(\mathrm{~d}, J=3.9 \mathrm{~Hz}), 139.8(\mathrm{~d}, J=4.8 \mathrm{~Hz}), 137.2(\mathrm{~d}, J=2.9 \mathrm{~Hz}), 136.6(\mathrm{~d}, J=1.9 \mathrm{~Hz})$, 134.97, 134.95, 134.6 (d, $J=5.1 \mathrm{~Hz}), 134.3$ (d, $J=2.9 \mathrm{~Hz}), 134.0$ (d, $J=3.1 \mathrm{~Hz}), 133.4$ (d, $J=9.1 \mathrm{~Hz}), 133.3,131.8$ (d, $J=2.9 \mathrm{~Hz}), 131.7$ (d, $\left.J=133.3 \mathrm{~Hz}, 3 \mathbf{u}^{\prime}\right), 131.64$ (d, $J$ $=134.8 \mathrm{~Hz}, 3 \mathbf{u}), 131.58,131.4,131.1(\mathrm{~d}, J=5.3 \mathrm{~Hz}), 130.7,130.4,129.3,128.9(\mathrm{~d}, J$ $=2.3 \mathrm{~Hz}), 128.7,128.5(\mathrm{~d}, J=4.1 \mathrm{~Hz}), 128.0(\mathrm{~d}, J=2.0 \mathrm{~Hz}), 127.80,127.75,127.7$, 127.6, $127.4(\mathrm{~d}, J=6.8 \mathrm{~Hz}), 127.3,126.8(\mathrm{~d}, J=1.6 \mathrm{~Hz}), 126.6(\mathrm{~d}, J=3.4 \mathrm{~Hz}), 126.4$ (d, $J=3.5 \mathrm{~Hz}), 125.8$ (d, $J=1.9 \mathrm{~Hz}), 125.54,125.48,125.3,125.2,120.90,120.85$, $120.1(\mathrm{~d}, J=2.0 \mathrm{~Hz}), 62.5$ (d, $\left.J=3.3 \mathrm{~Hz}, 3 \mathbf{u}^{\prime}\right), 62.0$ (d, $\left.J=3.4 \mathrm{~Hz}, 3 \mathbf{u}\right), 51.2$ (d, $J=$ $\left.79.2 \mathrm{~Hz}, \mathbf{3} \mathbf{u}^{\prime}\right), 51.0$ (d, $\left.J=78.9 \mathrm{~Hz}, \mathbf{3 u}\right), 21.3$ (3u'), 21.0 (3u), 20.6. ${ }^{31} \mathrm{P}$ NMR (162 
$\left.\mathrm{MHz}, \mathrm{CDCl}_{3}\right) \delta 28.6\left(3 \mathbf{u}^{\prime}\right), 28.4(3 \mathbf{u})$. HRMS (ESI) calcd for $\mathrm{C}_{34} \mathrm{H}_{29} \mathrm{NO}_{2} \mathrm{P}^{+}[\mathrm{M}+\mathrm{H}]^{+} m / z$ : 514.1930; found 514.1932.

Rel-(4bS,15S,16S)-16-(4-chlorophenyl)-11-methyl-15-phenyl-4b,16-dihydrodibenzo[b,f]benzo[4,5][1,2] azaphosphinino[1,6-d][1,4]oxazepine 15-oxide (3v) and rel-(4bS,15S,16S)-3-chloro-11-methyl-15,16-diphenyl-4b,16-dihydrodibenzo $[b, f]$ benzo $[4,5][1,2]$ azaphosphinino $[1,6-d][1,4]$ oxazepine 15-oxide (3v')

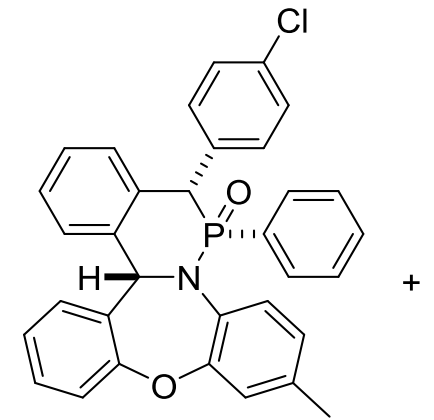

$3 v$

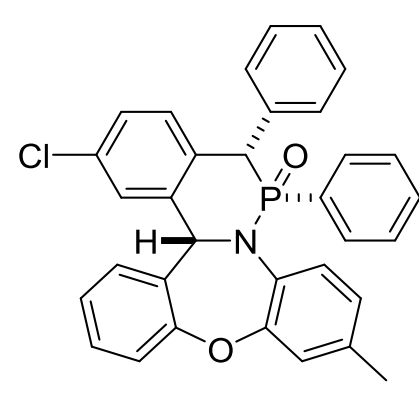

$3 \mathbf{v}^{\prime}$

White solid (62 mg, 56\%); mp. 292-294 ${ }^{\circ} \mathrm{C}$. IR (KBr) $v\left(\mathrm{~cm}^{-1}\right)$ 2974, 2926, 1504, 1491, 1529, 1214, 1090, 1049, 881, 734, 696. ${ }^{1} \mathrm{H}$ NMR $\left(400 \mathrm{MHz}, \mathrm{CDCl}_{3}\right) \delta 7.55(\mathrm{~d}, J=2.4 \mathrm{~Hz}, 1 \mathrm{H}), 7.49-7.07$ (m, 15H), $6.95(\mathrm{~d}, J=8.0 \mathrm{~Hz}, 1 \mathrm{H}), 6.80-6.75(\mathrm{~m}, 1 \mathrm{H}), 6.70-6.63(\mathrm{~m}, 1 \mathrm{H}), 6.63-6.55(\mathrm{~m}, 1 \mathrm{H})$, $6.30(\mathrm{dd}, J=8.0,2.8 \mathrm{~Hz}, 1 \mathrm{H}), 5.08\left(\mathrm{~d}, J=27.9 \mathrm{~Hz}, 0.31 \times 1 \mathrm{H}, \mathbf{3} \mathbf{v}^{\prime}\right), 5.07$ (d, $J=28.7$ $\mathrm{Hz}, 0.69 \times 1 \mathrm{H}, \mathbf{3 v}), 2.02\left(\mathrm{~s}, 0.31 \times 3 \mathrm{H}, 3 \mathbf{3} \mathbf{v}^{\prime}\right), 2.00(\mathrm{~s}, 0.69 \times 3 \mathrm{H}, 3 \mathrm{v}) .{ }^{13} \mathrm{C}$ NMR $(101$ $\left.\mathrm{MHz}, \mathrm{CDCl}_{3}\right) \delta 159.7,159.5,151.3(\mathrm{~d}, J=5.1 \mathrm{~Hz}), 142.5(\mathrm{~d}, J=3.8 \mathrm{~Hz}), 135.2,133.7$ (d, $J=3.2 \mathrm{~Hz}), 133.49$ (d, $J=9.3 \mathrm{~Hz}), 133.46$ (d, $J=9.1 \mathrm{~Hz}), 133.14,133.11$ (d, $J=$ $1.7 \mathrm{~Hz}), 132.5$ (d, $J=5.2 \mathrm{~Hz}), 132.2(\mathrm{~d}, J=2.8 \mathrm{~Hz}), 131.6,131.5,131.4,131.3(\mathrm{~d}, J=$ $5.5 \mathrm{~Hz}), 131.2,128.8(\mathrm{~d}, J=6.9 \mathrm{~Hz}), 128.4(\mathrm{~d}, J=133.4 \mathrm{~Hz}), 128.3(\mathrm{~d}, J=1.9 \mathrm{~Hz})$, 128.2, 128.04 (d, $J=1.6 \mathrm{~Hz}), 128.01$ (d, $J=13.3 \mathrm{~Hz}), 127.9$ (d, $J=13.7 \mathrm{~Hz}), 127.2$ (d, $J=1.5 \mathrm{~Hz}), 127.1(\mathrm{~d}, J=6.6 \mathrm{~Hz}), 126.6(\mathrm{~d}, J=3.6 \mathrm{~Hz}), 126.5(\mathrm{~d}, J=3.4 \mathrm{~Hz}), 126.4$ (d, $J=2.1 \mathrm{~Hz}), 125.8,125.7,125.6,125.4,121.2,121.1,120.30,120.26,62.4$ (d, $J=2.9$ Hz, 3v'), 61.8 (d, $J=3.4 \mathrm{~Hz}, 3 \mathbf{v}), 51.2$ (d, $J=78.4 \mathrm{~Hz}, 3 \mathbf{v}), 51.0$ (d, $J=78.7 \mathrm{~Hz}, \mathbf{3 v}$ '), 20.7. ${ }^{31} \mathrm{P}$ NMR (162 MHz, $\left.\mathrm{CDCl}_{3}\right) \delta 28.1$ (3v), 27.8 (3v'). HRMS (ESI) calcd for $\mathrm{C}_{33} \mathrm{H}_{26} \mathrm{ClNO}_{2} \mathrm{P}^{+}[\mathrm{M}+\mathrm{H}]^{+} m / z$ : 534.1384; found 534.1385. 
Rel-(4bS,15S,16S)-15-phenyl-16-(4-(trifluoromethyl)phenyl)-4b,16-dihydrodibenzo $[b, f]$ benzo $[4,5][1,2]$ azaphosphinino $[1,6-d][1,4]$ oxazepine 15-oxide (3w) and (4bS,15S,16S)-15,16-diphenyl-3-(trifluoromethyl)-4b,16-dihydrodibenzo $[b, f]$ benzo $[4,5][1,2]$ azaphosphinino $[1,6-d][1,4]$ oxazepine 15-oxide (3w')

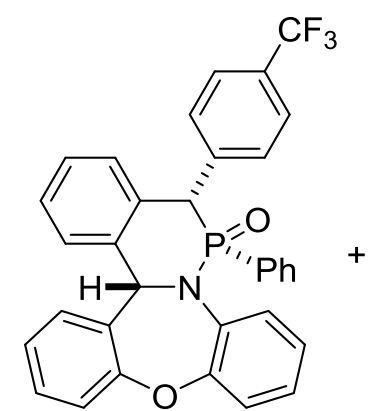

$3 w$

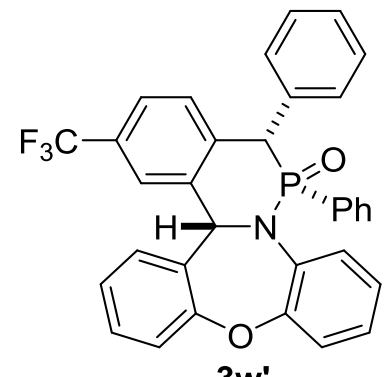

$3 w^{\prime}$

White solid (26 mg, 47\%); mp. 239-240 ${ }^{\circ} \mathrm{C}$. IR $(\mathrm{KBr}) v\left(\mathrm{~cm}^{-1}\right) 3394,3059,2956,2925,2853,1599,1491,1259,1208,1117$, 696. ${ }^{1} \mathrm{H}$ NMR (400 MHz, $\left.\mathrm{CDCl}_{3}\right) \delta 7.76(\mathrm{~d}, J=7.7 \mathrm{~Hz}, 1 \mathrm{H}), 7.55-7.30(\mathrm{~m}, 7 \mathrm{H}), 7.28$ $-7.04(\mathrm{~m}, 8 \mathrm{H}), 6.93-6.71(\mathrm{~m}, 5 \mathrm{H}), 6.37$ (d, $J=14.9 \mathrm{~Hz}, 1 \mathrm{H}, \mathbf{3 w}), 6.35$ (d, $J=14.3$ $\left.\mathrm{Hz}, 1 \mathrm{H}, 3 \mathbf{w}^{\prime}\right), 5.18\left(\mathrm{~d}, J=27.8 \mathrm{~Hz}, 1 \mathrm{H}, \mathbf{3} \mathbf{w}^{\prime}\right), 5.15$ (d, $\left.J=28.9,1 \mathrm{H}, \mathbf{3 w}\right) .{ }^{13} \mathrm{C} \mathrm{NMR}(101$ $\left.\mathrm{MHz}, \mathrm{CDCl}_{3}\right) \delta 159.8,153.7(\mathrm{~d}, J=5.0 \mathrm{~Hz}), 141.8(\mathrm{~d}, J=3.5 \mathrm{~Hz}), 138.9(\mathrm{~d}, J=3.5$ Hz), 134.3 (d, $J=3.3 \mathrm{~Hz}), 133.7,133.69,133.6,133.64,132.5$ (d, $J=2.8 \mathrm{~Hz}), 131.7$ $(\mathrm{d}, J=22.7 \mathrm{~Hz}), 131.5(\mathrm{~d}, J=5.4 \mathrm{~Hz}), 131.2(\mathrm{~d}, J=3.8 \mathrm{~Hz}), 128.6(\mathrm{~d}, J=2.1 \mathrm{~Hz})$, $128.3(\mathrm{~d}, J=13.2 \mathrm{~Hz}), 128.1$ (d, $J=2.8 \mathrm{~Hz}), 128.0$ (d, $J=6.6 \mathrm{~Hz}), 127.68,126.70$ (d, $J=6.5 \mathrm{~Hz}), 126.2(\mathrm{~d}, J=3.6 \mathrm{~Hz}), 126.8\left(\mathrm{q}, J_{C-F}=273.7 \mathrm{~Hz}\right), 125.5(\mathrm{~d}, J=14.3 \mathrm{~Hz})$, $122.9\left(\mathrm{q}, J_{C-F}=4.56 \mathrm{~Hz}\right), 121.2(\mathrm{~d}, J=50.0 \mathrm{~Hz}), 61.9(\mathrm{~d}, J=3.0 \mathrm{~Hz}), 51.8(\mathrm{~d}, J=77.9$ Hz). ${ }^{19} \mathrm{~F}$ NMR (376 MHz, $\left.\mathrm{CDCl}_{3}\right) \delta-62.43$ (3w), -62.64 (3w'). ${ }^{31} \mathrm{P}$ NMR (162 MHz, $\left.\mathrm{CDCl}_{3}\right) \delta$ 27.80. HRMS (ESI) calcd for $\mathrm{C}_{33} \mathrm{H}_{24} \mathrm{~N}_{2} \mathrm{OP}^{+}[\mathrm{M}+\mathrm{H}]^{+} m / z: 554.1491$; found 554.1498 .

Rel-(4bS,15S,16S)-16-(4-methoxyphenyl)-15-phenyl-4b,16-dihydrodibenzo[b, $f]$ benzo $[4,5][1,2]$ azaphosphinino[1,6- $d][1,4]$ oxazepine 15-oxide $(3 x)$ and (4bS,15S,16S)-3-methoxy-15,16-diphenyl-4b,16-dihydrodibenzo[b, $f]$ benzo[4,5] $[1,2]$ azaphosphinino[1,6- $d][1,4]$ oxazepine 15-oxide (3x') 


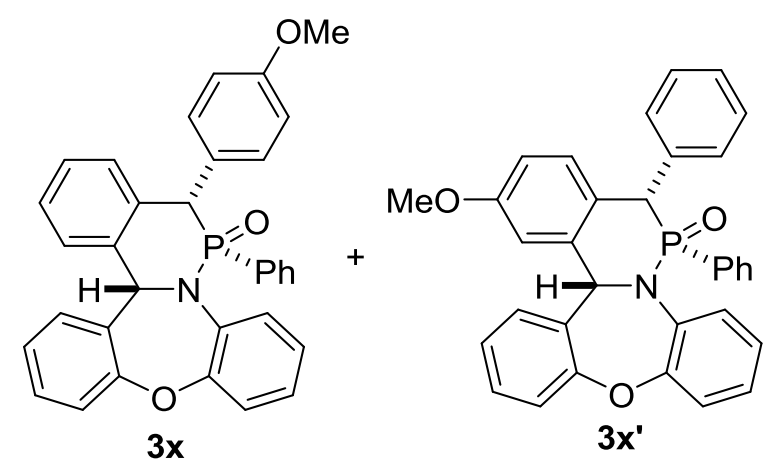

Trace amount. Only observed in its reaction mixture by HRMS. HRMS (ESI) calcd for $\mathrm{C}_{33} \mathrm{H}_{27} \mathrm{NO}_{3} \mathrm{P}^{+}[\mathrm{M}+\mathrm{H}]^{+} m / z$ : 516.1723; found 516.1724.

Rel-(4bS,15S,16S)-11-methyl-15,16-di(4-methylphenyl)-4b,16-dihydrodibenzo $[b, f]$ benzo[4,5][1,2] azaphosphinino[1,6-d][1,4]oxazepine 15-oxide (3y) and rel(4bS,15S,16S)-3,11-dimethyl-15-(4-methylphenyl)-16-phenyl-4b,16-dihydrodibenzo $[b, f]$ benzo $[4,5][1,2]$ azaphosphinino[1,6- $d][1,4]$ oxazepine 15-oxide (3y')

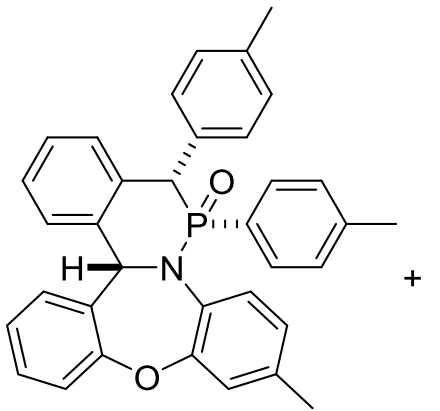

$3 y$

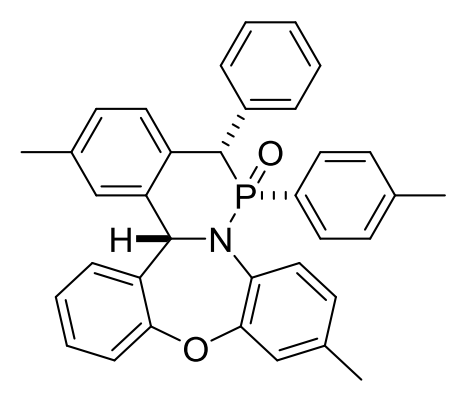

$3 \mathbf{y}^{\prime}$

White solid (40 mg, 39\%); mp.

284-286 ${ }^{\circ} \mathrm{C} . \mathrm{IR}(\mathrm{KBr}) v\left(\mathrm{~cm}^{-1}\right)$ 3030, 2922, 2859, 1601, 1505, 1491, 1264, 1215, 1112 , 988, 908, 811, 731, 571, 521. ${ }^{1} \mathrm{H}$ NMR $\left(400 \mathrm{MHz}, \mathrm{CDCl}_{3}\right) \delta 7.60$ (s, 1H), $7.46-7.34$ (m, 3H), $7.26-7.18(\mathrm{~m}, 3 \mathrm{H}), 7.15-7.04(\mathrm{~m}, 4 \mathrm{H}), 6.99-6.89(\mathrm{~m}, 4 \mathrm{H}), 6.76-6.54(\mathrm{~m}$, 4H), $6.38-6.24(\mathrm{~m}, 1 \mathrm{H}), 5.08\left(\mathrm{~d}, J=28.4 \mathrm{~Hz}, 1 \mathrm{H} \times 0.64,3 \mathbf{y}^{\prime}\right), 5.02(\mathrm{~d}, J=27.4 \mathrm{~Hz}$, $1 \mathrm{H} \times 0.36,3 \mathbf{y}), 2.32(\mathrm{~s}, 3 \mathrm{H}), 2.25(\mathrm{~s}, 3 \mathrm{H}), 2.04\left(\mathrm{~s}, 3 \mathrm{H} \times 0.64,3 \mathbf{y}^{\prime}\right), 2.02(\mathrm{~s}, 3 \mathrm{H} \times 0.36$, 3y). ${ }^{13} \mathrm{C}$ NMR $\left(101 \mathrm{MHz}, \mathrm{CDCl}_{3}\right) \delta 159.8,159.2,151.3(\mathrm{~d}, J=4.7 \mathrm{~Hz}), 142.3$ (d, $J=$ $2.9 \mathrm{~Hz}), 140.6$ (d, $J=4.0 \mathrm{~Hz}), 140.0(\mathrm{~d}, J=4.8 \mathrm{~Hz}), 137.1$ (d, $J=3.0 \mathrm{~Hz}), 136.6$ (d, $J$ $=1.8 \mathrm{~Hz}), 135.0,134.7(\mathrm{~d}, J=5.0 \mathrm{~Hz}), 134.5(\mathrm{~d}, J=3.1 \mathrm{~Hz}), 134.1(\mathrm{~d}, J=3.4 \mathrm{~Hz})$, 133.6, $133.5(\mathrm{~d}, J=2.3 \mathrm{~Hz}), 133.4,132.5(\mathrm{~d}, J=4.2 \mathrm{~Hz}), 131.6,131.4,131.18(\mathrm{~d}, J=$ $11.1 \mathrm{~Hz}), 131.18,130.7,130.4,128.9$ (d, $J=2.1 \mathrm{~Hz}), 128.63$ (d, $J=13.7 \mathrm{~Hz}), 128.58$ $(\mathrm{d}, J=13.3 \mathrm{~Hz}), 128.0(\mathrm{~d}, J=6.1 \mathrm{~Hz}), 127.91(\mathrm{~d}, J=2.4 \mathrm{~Hz}), 127.85,127.34(\mathrm{~d}, J=$ 
$6.7 \mathrm{~Hz}), 127.25(\mathrm{~d}, J=1.8 \mathrm{~Hz}), 127.2(\mathrm{~d}, J=2.8 \mathrm{~Hz}), 126.7,126.6(\mathrm{~d}, J=3.4 \mathrm{~Hz})$, $125.9(\mathrm{~d}, J=13.8 \mathrm{~Hz}), 125.8(\mathrm{~d}, J=1.6 \mathrm{~Hz}), 124.7,124.5,120.90,120.85$, 120.1, 62.5 (d, $J=3.0 \mathrm{~Hz}, 3 \mathbf{y}), 62.1$ (d, $J=3.2 \mathrm{~Hz}, 3 \mathbf{y}$ '), 51.3 (d, $J=79.4 \mathrm{~Hz}, 3 \mathbf{y}), 51.1$ (d, $J=79.1$ Hz, 3y'), 21.5 (3y'), 21.3 (3y), 21.02 (3y'), 20.98 (3y), 20.6, 14.1. ${ }^{31}$ P NMR (162 MHz, $\left.\mathrm{CDCl}_{3}\right) \delta 29.0(\mathbf{3 y}), 28.8\left(\mathbf{3 y} \mathbf{y}^{\prime}\right)$. HRMS (ESI) calcd for $\mathrm{C}_{35} \mathrm{H}_{31} \mathrm{NO}_{2} \mathrm{P}^{+}[\mathrm{M}+\mathrm{H}]^{+} \mathrm{m} / z$ : 528.2087; found 528.2086.

Rel-(4bS,15S,16S)-15,16-bis(4-chlorophenyl)-11-methyl-4b,16-dihydrodibenzo $[b, f]$ benzo[4,5][1,2] azaphosphinino[1,6-d][1,4]oxazepine 15-oxide (3z) and rel(4bS,15S,16S)-3-chloro-15-(4-chlorophenyl)-11-methyl-16-phenyl-4b,16-dihydrodibenzo $[b, f]$ benzo $[4,5][1,2]$ azaphosphinino $[1,6-d][1,4]$ oxazepine 15-oxide $\left(3 z^{\prime}\right)$

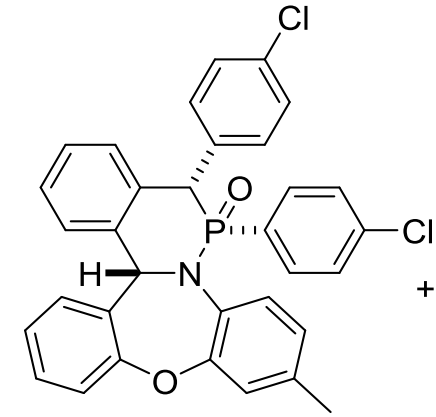

$3 z$

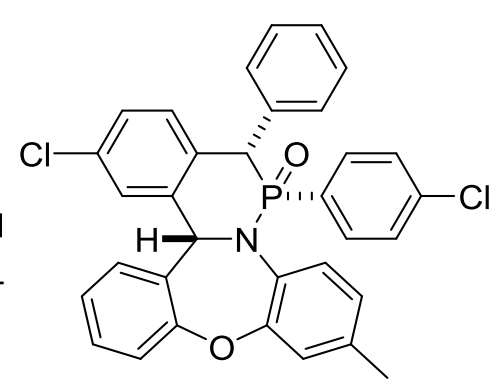

$3 z^{\prime}$

White solid (107 mg, 94\%); mp. 284-286 ${ }^{\circ} \mathrm{C} . \mathrm{IR}(\mathrm{KBr}) v\left(\mathrm{~cm}^{-1}\right) 2923,1507,1491,1214,1120,1086,821,699,571$, 540. ${ }^{1} \mathrm{H}$ NMR $\left(400 \mathrm{MHz}, \mathrm{CDCl}_{3}\right) \delta 7.55(\mathrm{~s}, 1 \mathrm{H}), 7.48-7.09(\mathrm{~m}, 13 \mathrm{H}), 7.00-6.94(\mathrm{~m}$, $1 \mathrm{H}), 6.81-6.74(\mathrm{~m}, 1 \mathrm{H}), 6.73-6.60(\mathrm{~m}, 3 \mathrm{H}), 6.33-6.23(\mathrm{~m}, 1 \mathrm{H}), 5.09(\mathrm{~d}, J=28.0$ $\mathrm{Hz}, 0.60 \times 1 \mathrm{H}, \mathbf{3 z}), 5.07\left(\mathrm{~d}, J=28.7 \mathrm{~Hz}, 0.40 \times 1 \mathrm{H}, \mathbf{3 z} \mathbf{z}^{\prime}\right), 2.05(\mathrm{~s}, 0.60 \times 3 \mathrm{H}, \mathbf{3 z})$, $2.03\left(\mathrm{~s}, 0.40 \times 3 \mathrm{H}, \mathbf{3 z}\right.$ '). ${ }^{13} \mathrm{C} \mathrm{NMR}\left(101 \mathrm{MHz}, \mathrm{CDCl}_{3}\right) \delta 159.4$ (3z'), 159.2 (3z), 151.4 (d, $J=5.1 \mathrm{~Hz}), 142.2(\mathrm{~d}, J=4.1 \mathrm{~Hz}), 139.9(\mathrm{~d}, J=4.9 \mathrm{~Hz}), 138.8(\mathrm{~d}, J=3.9 \mathrm{~Hz}), 138.8$ (d, $J=4.0 \mathrm{~Hz}), 135.3,134.8(\mathrm{~d}, J=10.0 \mathrm{~Hz}), 134.8(\mathrm{~d}, J=10.1 \mathrm{~Hz}), 133.8(\mathrm{~d}, J=2.9$ Hz), $133.6(\mathrm{~d}, J=3.6 \mathrm{~Hz}), 133.5$ (d, $J=3.0 \mathrm{~Hz}), 133.4$ (d, $J=5.5 \mathrm{~Hz}), 133.2$ (d, $J=2.4$ $\mathrm{Hz}), 132.6$ (d, $J=5.3 \mathrm{~Hz}), 132.3(\mathrm{~d}, J=5.4 \mathrm{~Hz}), 131.5,131.4,131.3(\mathrm{~d}, J=4.6 \mathrm{~Hz})$, 131.16, 131.15 (d, $J=5.2 \mathrm{~Hz}), 130.7,130.6(\mathrm{~d}, J=4.6 \mathrm{~Hz}), 128.44,128.42,128.37$ (d, $J=131.8 \mathrm{~Hz}, \mathbf{3 z}), 128.36,128.30,128.29$ (d, $J=133.1 \mathrm{~Hz}, \mathbf{3 z}$ '), 128.28, 128.1, 127.9 (d, $J=2.9 \mathrm{~Hz}), 127.8,127.62,127.55,127.2(\mathrm{~d}, J=1.5 \mathrm{~Hz}), 126.9(\mathrm{~d}, J=6.6 \mathrm{~Hz})$, $126.8(\mathrm{~d}, J=1.7 \mathrm{~Hz}), 126.6(\mathrm{~d}, J=3.7 \mathrm{~Hz}), 126.5(\mathrm{~d}, J=3.4 \mathrm{~Hz}), 126.4(\mathrm{~d}, J=8.1 \mathrm{~Hz})$, 
126.2, 126.0, 125.7, 125.4, 121.2, 121.1, 120.38, 120.36, 62.5 (d, $J=3.3 \mathrm{~Hz}, \mathbf{3 z}), 61.9$ (d, $J=3.5 \mathrm{~Hz}, \mathbf{3 z ^ { \prime }}$ ), 50.9 (d, $J=79.2 \mathrm{~Hz}, \mathbf{3 z}$ '), 50.8 (d, $\left.J=79.6 \mathrm{~Hz}, \mathbf{3 z}\right), 20.7 .{ }^{31} \mathrm{P}$ NMR $\left(162 \mathrm{MHz}, \mathrm{CDCl}_{3}\right) \delta 27.2\left(\mathbf{3 z} \mathbf{z}^{\prime}\right), 26.8(\mathbf{3 z})$. HRMS (ESI) calcd for $\mathrm{C}_{33} \mathrm{H}_{25} \mathrm{Cl}_{2} \mathrm{NO}_{2} \mathrm{P}^{+}$ $[\mathrm{M}+\mathrm{H}]^{+} \mathrm{m} / \mathrm{z}: 568.0994 ;$ found 568.0999.

$\operatorname{Rel-(4bS,15S,16S)-11-chloro-15,16-bis(4-chlorophenyl)-4b,16-dihydrodibenzo[}[b, f]$ benzo[4,5][1,2] azaphosphinino[1,6-d][1,4] oxazepine 15-oxide (3aa) and rel(4bS,15S,16S)-3,11-dichloro-15-(4-chlorophenyl)-16-phenyl-4b,16-dihydrodibenzo $[b, f]$ benzo $[4,5][1,2]$ azaphosphinino[1,6- $d][1,4]$ oxazepine 15-oxide (3aa')

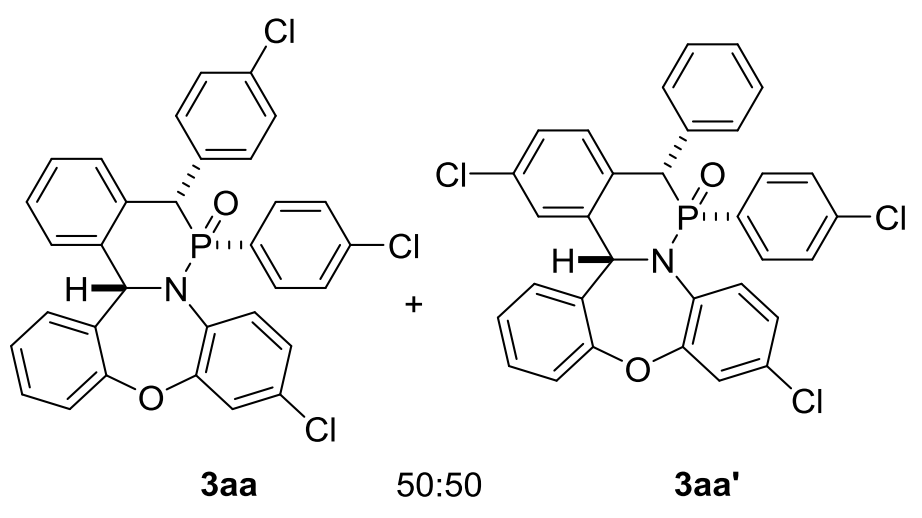

White solid (92 mg, 78\%); mp. 282-284 ${ }^{\circ} \mathrm{C}$. IR $(\mathrm{KBr}) v\left(\mathrm{~cm}^{-1}\right)$ 3063, 2928, 2852, 1736, 1582, 1482, 1238, 1210, 1085 , 1013, 978, 902, 823, 783, 742, 577, 549. ${ }^{1} \mathrm{H}$ NMR (400 MHz, $\left.\mathrm{CDCl}_{3}\right) \delta 7.76-7.68(\mathrm{~m}$, 1H), $7.54-7.39(\mathrm{~m}, 2 \mathrm{H}), 7.37-7.24(\mathrm{~m}, 4 \mathrm{H}), 7.22-7.05(\mathrm{~m}, 8 \mathrm{H}), 6.83-6.57(\mathrm{~m}$, 4H), $6.33(\mathrm{dd}, J=7.2,5.2 \mathrm{~Hz}, 1 \mathrm{H}), 5.16(\mathrm{~d}, J=28.5 \mathrm{~Hz}, 0.50 \times 1 \mathrm{H}), 5.13(\mathrm{~d}, J=29.0$ $\mathrm{Hz}, 0.50 \times 1 \mathrm{H}) .{ }^{13} \mathrm{C} \mathrm{NMR}\left(101 \mathrm{MHz}, \mathrm{CDCl}_{3}\right) \delta 159.0,158.9,153.8(\mathrm{~d}, J=3.0 \mathrm{~Hz})$, $153.7(\mathrm{~d}, J=2.9 \mathrm{~Hz}), 142.2(\mathrm{~d}, J=3.7 \mathrm{~Hz}), 140.0(\mathrm{~d}, J=4.1 \mathrm{~Hz}), 139.21(\mathrm{~d}, J=3.3$ $\mathrm{Hz}), 139.16$ (d, $J=3.3 \mathrm{~Hz}), 134.8$ (d, $J=9.8 \mathrm{~Hz}), 133.9$ (d, $J=3.4 \mathrm{~Hz}), 133.35,133.33$ $(\mathrm{d}, J=7.7 \mathrm{~Hz}), 133.0(\mathrm{~d}, J=3.2 \mathrm{~Hz}), 132.8$ (d, $J=3.2 \mathrm{~Hz}), 132.6(\mathrm{~d}, J=5.1 \mathrm{~Hz}), 132.4$ (d, $J=5.5 \mathrm{~Hz}), 131.7$ (d, $J=5.5 \mathrm{~Hz}), 131.5,131.2(\mathrm{~d}, J=5.5 \mathrm{~Hz}), 131.1,130.7$ (d, $J=$ $4.5 \mathrm{~Hz}), 130.0(\mathrm{~d}, J=4.5 \mathrm{~Hz}), 129.8$ (d, $J=8.7 \mathrm{~Hz}), 128.7,128.6,128.5$ (d, $J=1.9 \mathrm{~Hz})$, 128.4, 128.2, $128.1(\mathrm{~d}, J=133.3 \mathrm{~Hz}), 128.0(\mathrm{~d}, J=2.7 \mathrm{~Hz}), 127.4(\mathrm{~d}, J=1.6 \mathrm{~Hz}), 127.2$ $(\mathrm{d}, J=6.9 \mathrm{~Hz}), 126.8(\mathrm{~d}, J=3.1 \mathrm{~Hz}), 126.7$ (d, $J=3.2 \mathrm{~Hz}), 126.6(\mathrm{~d}, J=6.6 \mathrm{~Hz}), 126.4$, $126.3(\mathrm{~d}, J=1.8 \mathrm{~Hz}), 126.2$ (d, $J=2.4 \mathrm{~Hz}), 126.0,125.6$ (d, $J=2.5 \mathrm{~Hz}), 121.4,121.3$, 121.1, $62.1(\mathrm{~d}, J=3.3 \mathrm{~Hz}), 61.6(\mathrm{~d}, J=3.3 \mathrm{~Hz}), 50.8(\mathrm{~d}, J=79.1 \mathrm{~Hz}), 50.6(\mathrm{~d}, J=79.3$ 
Hz). ${ }^{31} \mathrm{P} \mathrm{NMR}\left(162 \mathrm{MHz}, \mathrm{CDCl}_{3}\right) \delta 27.8,27.5$. HRMS (ESI) calcd for $\mathrm{C}_{32} \mathrm{H}_{22} \mathrm{Cl}_{3} \mathrm{NO}_{2} \mathrm{P}^{+}$ $[\mathrm{M}+\mathrm{H}]^{+} m / z: 588.0448$; found 588.0448.

Rel-(4bS,15S,16R)-15-phenyl-16-(pyridin-3-yl)-4b,16-dihydrodibenzo $[b, f]$ benzo $[4,5][1,2]$ azaphosphinino[1,6- $d][1,4]$ oxazepine 15-oxide (3ab)

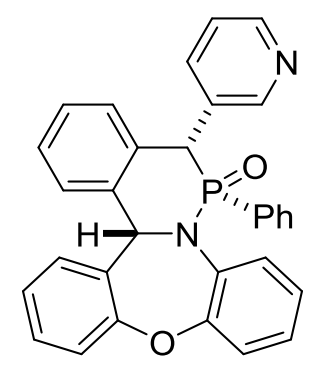

3ab White solid (14 mg, 29\%); mp. 245-246 ${ }^{\circ} \mathrm{C}$. IR (KBr) $v\left(\mathrm{~cm}^{-1}\right) 3357$, 3061, 2956, 2925, 2853, 1490, 1330, 1128, 736, 526. ${ }^{1} \mathrm{H}$ NMR (400 MHz, $\left.\mathrm{CDCl}_{3}\right) \delta$ 8.47 (d, $J=4.8 \mathrm{~Hz}, 1 \mathrm{H}), 8.08$ (s, 1H), 7.74 (d, $J=7.6 \mathrm{~Hz}, 1 \mathrm{H}), 7.49$ - 7.27 (m, 5H), $7.26-7.08(\mathrm{~m}, 6 \mathrm{H}), 7.09-6.99(\mathrm{~m}, 3 \mathrm{H}), 6.93(\mathrm{~d}, \mathrm{~J}=6.7 \mathrm{~Hz}, 1 \mathrm{H}), 6.84-6.69(\mathrm{~m}, 3 \mathrm{H})$, $6.33(\mathrm{~d}, J=7.7 \mathrm{~Hz}, 1 \mathrm{H}), 5.09(\mathrm{~d}, J=27.9 \mathrm{~Hz}, 1 \mathrm{H}) .{ }^{13} \mathrm{C} \mathrm{NMR}\left(101 \mathrm{MHz}, \mathrm{CDCl}_{3}\right) \delta$ 159.6, $153.6(\mathrm{~d}, J=5.1 \mathrm{~Hz}), 152.3(\mathrm{~d}, J=5.9 \mathrm{~Hz}), 148.9$ (d, $J=2.8 \mathrm{~Hz}), 140.5$ (d, $J=$ $4.3 \mathrm{~Hz}), 138.5$ (d, $J=4.7 \mathrm{~Hz}), 134.5$ (d, $J=3.4 \mathrm{~Hz}), 133.6$ (d, $J=9.1 \mathrm{~Hz}), 133.4$ (d, $J$ $=5.5 \mathrm{~Hz}), 132.7(\mathrm{~d}, J=2.9 \mathrm{~Hz}), 128.7,128.5(\mathrm{~d}, J=1.9 \mathrm{~Hz}), 128.3,127.7(\mathrm{~d}, J=6.6$ Hz), $127.5(\mathrm{~d}, J=1.6 \mathrm{~Hz}), 127.4(\mathrm{~d}, J=6.2 \mathrm{~Hz}), 126.6(\mathrm{~d}, J=1.7 \mathrm{~Hz}), 126.3(\mathrm{~d}, J=3.5$ Hz), 125.9, 125.6 (d, $J=6.1 \mathrm{~Hz}), 123.1$ (d, $J=1.8 \mathrm{~Hz}), 121.1$ (d, $J=37.4 \mathrm{~Hz}), 62.4$ (d, $J=3.4 \mathrm{~Hz}), 49.2(\mathrm{~d}, J=78.5 \mathrm{~Hz}) .{ }^{31} \mathrm{P} \mathrm{NMR}\left(162 \mathrm{MHz}, \mathrm{CDCl}_{3}\right) \delta 27.83 . \mathrm{HRMS}(\mathrm{ESI})$ calcd for $\mathrm{C}_{31} \mathrm{H}_{24} \mathrm{~N}_{2} \mathrm{O}_{2} \mathrm{P}^{+}[\mathrm{M}+\mathrm{H}]^{+} m / z$ : 487.1570; found 487.1575 .

\section{Procedure for Large-Scaled Preparation of Product 3b}

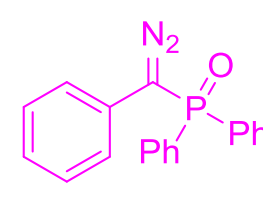

$4 \mathrm{mmol}$<smiles>Cc1ccc2c(c1)Oc1ccccc1C=N2</smiles>

$2 \mathrm{mmol}$

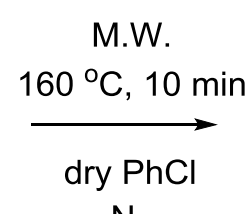

$\mathrm{N}_{2}$

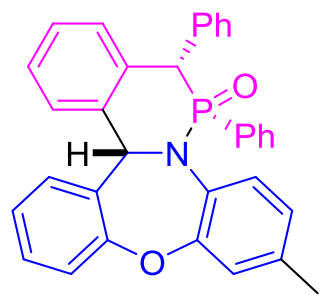

$700 \mathrm{mg}, 70 \%$ 
Diazo compound 1a (4 mmol, $1.27 \mathrm{~g}$ ) and cyclic imine $\mathbf{2 b}(2 \mathrm{mmol}, 0.42 \mathrm{~g}$ ) were dissolved in $20 \mathrm{~mL}$ of dry $\mathrm{PhCl}$ in a $35 \mathrm{~mL}$ microwave reaction tube. Then the resultant mixture was stirred in a microwave reactor at $160{ }^{\circ} \mathrm{C}$ for $10 \mathrm{~min}$. After cooling to room temperature, $\mathrm{PhCl}$ was removed, and the reaction mixture was directly subjected to flash column chromatography (PE:EA $=4: 1$ to $1: 1, v / v)$ to afford product $\mathbf{3 b}(700 \mathrm{mg}$, $70 \%)$.

\section{Procedure for the Suzuki Coupling Reaction of Compound 3g}

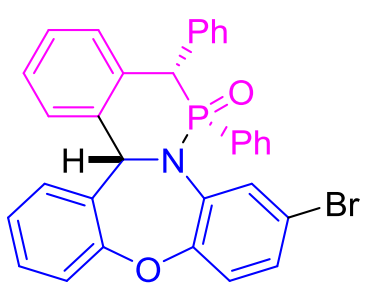

$3 \mathbf{g}$

$0.1 \mathrm{mmol}$

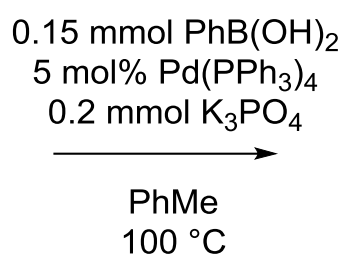

$100{ }^{\circ} \mathrm{C}$

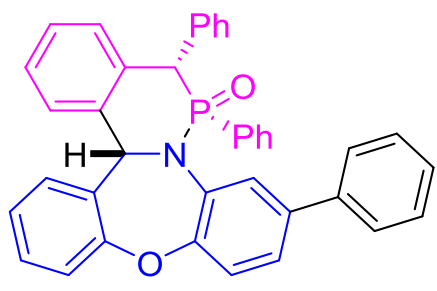

$4,59 \%$

Compound 3g (0.1 mmol, $56 \mathrm{mg}), \mathrm{PhB}(\mathrm{OH})_{2}(0.15 \mathrm{mmol}, 19.8 \mathrm{mg}), \mathrm{Pd}\left(\mathrm{PPh}_{3}\right)_{4}(5$ mol\%, $7.8 \mathrm{mg})$, and $\mathrm{K}_{3} \mathrm{PO}_{4}(0.2 \mathrm{mmol}, 43 \mathrm{mg})$ were added into a $8 \mathrm{~mL}$ reaction tube. The tube was charged with $\mathrm{N}_{2}$ and added $1.5 \mathrm{~mL}$ dry $\mathrm{PhMe}$. Then the resultant mixture was stirred in a classic oil bath for $18 \mathrm{~h}$ at $100{ }^{\circ} \mathrm{C}$. After cooling to room temperature, the reaction was washed with water, extracted with EA, dried over anhydrous $\mathrm{Na}_{2} \mathrm{SO}_{4}$. Then the mixture was subjected to flash column chromatography (PE:EA $=5: 1$ to 1:1, $v / v)$ to afford product 4 (33 $\mathrm{mg}, 59 \%)$.

\section{Rel-(4bS,15S,16S)-12,15,16-triphenyl-4b,16-dihydrodibenzo $[b, f]$ benzo $[4,5][1,2]$} azaphosphinino[1,6- $d][1,4]$ oxazepine 15-oxide (4)<smiles>O=P1(c2ccccc2)[C@H](c2ccccc2)c2ccccc2C12c1ccccc1Oc1ccc(-c3ccccc3)cc12</smiles>

White solid (33 mg, 59\%); mp. $294-296{ }^{\circ} \mathrm{C} .{ }^{1} \mathrm{H}$ NMR (400 $\left.\mathrm{MHz}, \mathrm{CDCl}_{3}\right) \delta 8.05(\mathrm{~d}, J=2.1 \mathrm{~Hz}, 1 \mathrm{H}), 7.48-7.37(\mathrm{~m}, 4 \mathrm{H}), 7.28-7.13(\mathrm{~m}, 14 \mathrm{H})$, $7.11-7.06(\mathrm{~m}, 3 \mathrm{H}), 6.98(\mathrm{dd}, J=8.3,2.2 \mathrm{~Hz}, 1 \mathrm{H}), 6.80(\mathrm{~d}, J=7.5 \mathrm{~Hz}, 2 \mathrm{H}), 6.73-6.66$ $(\mathrm{m}, 1 \mathrm{H}), 6.37(\mathrm{~d}, J=7.8 \mathrm{~Hz}, 1 \mathrm{H}), 5.14(\mathrm{~d}, J=28.7 \mathrm{~Hz}, 1 \mathrm{H}) .{ }^{13} \mathrm{C}$ NMR $(101 \mathrm{MHz}$, 
$\left.\mathrm{CDCl}_{3}\right) \delta 159.6,152.8(\mathrm{~d}, J=4.6 \mathrm{~Hz}), 140.5(\mathrm{~d}, J=3.9 \mathrm{~Hz}), 139.6,138.2,134.6(\mathrm{~d}, J$ $=2.8 \mathrm{~Hz}), 134.2(\mathrm{~d}, J=5.0 \mathrm{~Hz}), 134.1,133.4(\mathrm{~d}, J=9.1 \mathrm{~Hz}), 132.12(\mathrm{~d}, J=2.0 \mathrm{~Hz})$, 131.79, $131.6(\mathrm{~d}, J=4.1 \mathrm{~Hz}), 131.4(\mathrm{~d}, J=5.5 \mathrm{~Hz}), 130.9,128.8(\mathrm{~d}, J=83.3 \mathrm{~Hz}), 128.5$, 128.2, 128.1 (d, $J=2.2 \mathrm{~Hz}), 127.9,127.67$ (d, $J=12.9 \mathrm{~Hz}), 127.66,127.5$ (d, $J=6.7$ Hz), 127.1, 127.0, 126.7, 126.0, 125.8, 124.6 (d, $J=2.9$ Hz), 123.4, 121.1, 120.9, 62.1 $(\mathrm{d}, J=2.5 \mathrm{~Hz}), 51.4(\mathrm{~d}, J=78.3 \mathrm{~Hz}) .{ }^{31} \mathrm{P} \mathrm{NMR}\left(162 \mathrm{MHz}, \mathrm{CDCl}_{3}\right) \delta 29.0 . \mathrm{HRMS}(\mathrm{ESI})$ calcd for $\mathrm{C}_{38} \mathrm{H}_{29} \mathrm{NO}_{2} \mathrm{P}^{+}[\mathrm{M}+\mathrm{H}]^{+} m / z$ : 562.1930; found 562.1934.

\section{Crystal Data and Structure Refinement for Compound 3c}

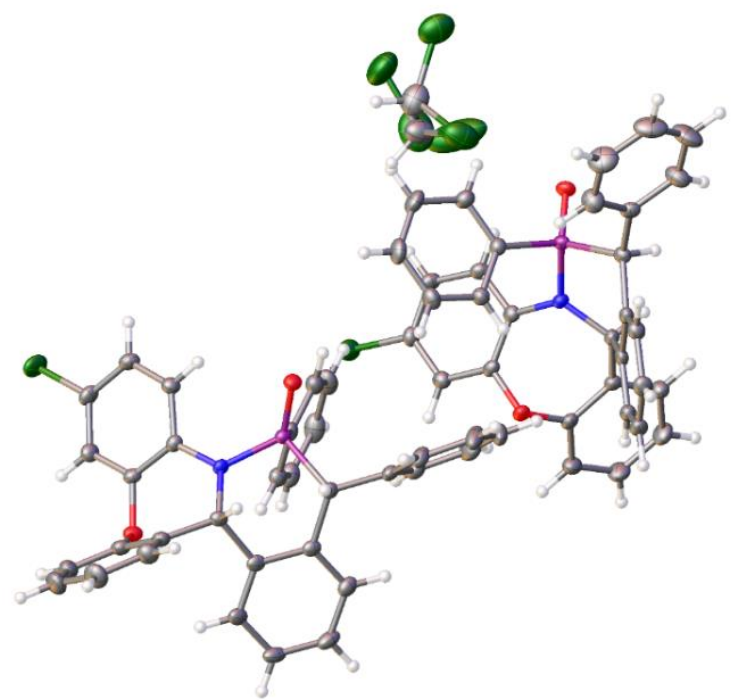

Table 1: Crystal data and structure refinement for compound 3c

Empirical formula

Formula weight

Temperature / $\mathrm{K}$

Crystal system

Space group

$\mathrm{a} / \AA, \mathrm{b} / \AA, \mathrm{c} / \AA$

$\alpha /^{\circ}, \beta /{ }^{\circ}, \gamma /{ }^{\circ}$

Volume / $\AA^{3}$

Z
$\mathrm{C}_{32.5} \mathrm{H}_{23.5} \mathrm{Cl}_{2.5} \mathrm{NO}_{2} \mathrm{P}$

579.62

$110.55(10)$

triclinic

P-1

10.3590(3), 13.9540(4), 20.3165(6)

95.329(3), 100.467(3), 105.604(3)

2750.12(15)

4 


$\begin{array}{ll}\rho_{\text {calc }} / \mathrm{mg} \mathrm{mm}^{-3} & 1.400 \\ \mu / \mathrm{mm}^{-1} & 0.375 \\ \mathrm{~F}(000) & 1196 \\ \text { Crystal size } / \mathrm{mm}^{3} & 0.30 \times 0.25 \times 0.24 \\ 2 \Theta \text { range for data collection } & 6.14 \text { to } 52^{\circ} \\ \text { Index ranges } & -12 \leq \mathrm{h} \leq 12,-17 \leq \mathrm{k} \leq 17,-25 \leq 1 \leq 25 \\ \text { Reflections collected } & 24600 \\ \text { Independent reflections } & 10783[\mathrm{R}(\mathrm{int})=0.0324(\mathrm{inf}-0.9 \AA)] \\ \text { Data/restraints/parameters } & 10783 / 57 / 731 \\ \text { Goodness-of-fit on } \mathrm{F}^{2} & 1.029 \\ \text { Final R indexes [I }>2 \sigma(\mathrm{I}) \text { i.e. } \mathrm{F}_{\mathrm{o}}>4 \sigma & \mathrm{R}_{1}=0.0471, \mathrm{wR}_{2}=0.1036 \\ \left.\left(\mathrm{~F}_{\mathrm{o}}\right)\right] & \mathrm{R}_{1}=0.0600, \mathrm{wR}_{2}=0.1118 \\ \text { Final R indexes [all data] } & 0.662 /-0.650 \\ \text { Largest diff. peak/hole } / \mathrm{e} \AA^{-3} & \mathrm{~N} \\ \text { Flack Parameters } & 0.9980 \\ \text { Completeness } & \end{array}$

\section{Experimental}

Pure product 3c recrystallized from ethyl acetate was dissolved in $\mathrm{CHCl}_{3}$ in a small test tube. The tube was kept in a cool place to allow the solvent to evaporate slowly until the crystals were grown. One of single crystals of $\mathrm{C}_{32.5} \mathrm{H}_{23.5} \mathrm{Cl}_{2.5} \mathrm{NO}_{2} \mathrm{P}$ [compond $3 \mathbf{c}\left(\mathrm{CHCl}_{3}\right.$ solvent)] was selected and transferred to the diffractometer. 


\section{Competitive Experiments and Copies of NMR Spectra}

1a $(31.8 \mathrm{mg}, 0.1 \mathrm{mmol}), 2 \mathrm{a}(9.75 \mathrm{mg}, 0.05 \mathrm{mmol})$, and $2(0.05 \mathrm{mmol})$ in $1.5 \mathrm{~mL}$ of anhydrous $\mathrm{PhCl}$ under $\mathrm{N}_{2}$ atmosphere were heated under microwave irradiation at $160{ }^{\circ} \mathrm{C}$ for $10 \mathrm{~min}(200 \mathrm{~W})$. The product ratio of $\mathbf{3 a}$ and $\mathbf{3}$ was determined based on the mixture ${ }^{1} \mathrm{H}$ NMR and ${ }^{31} \mathrm{P} \mathrm{NMR}$ analyses in $\mathrm{CDCl}_{3}$.

\section{Copies of NMR spectra for the reaction mixture of 1a, 2a, and 2}

${ }^{1} \mathrm{H}$ NMR for the reaction of $\mathbf{2 a}$ and $\mathbf{2 b}$ in $\mathrm{CDCl}_{3}(400 \mathrm{MHz})$

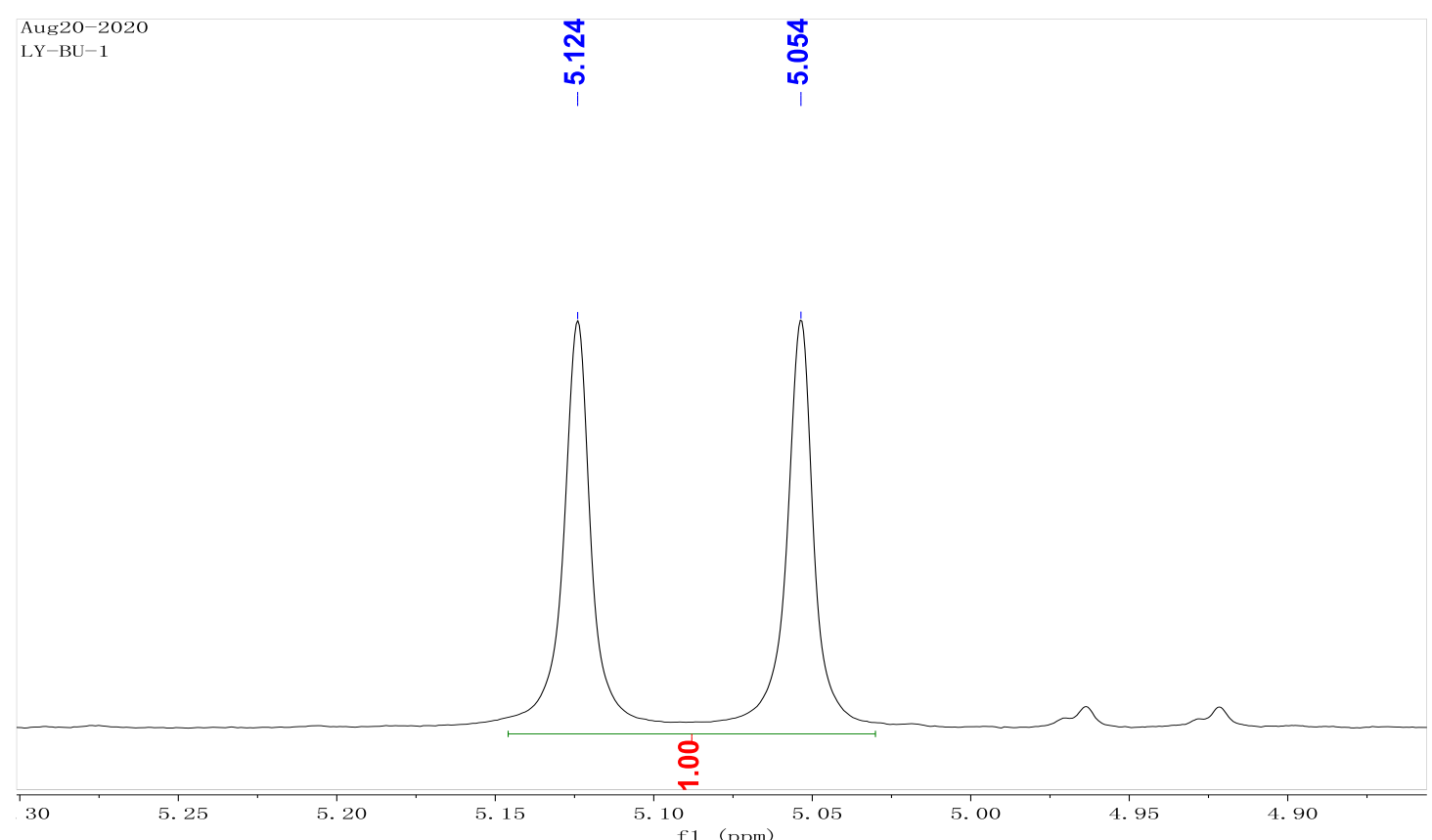

${ }^{31} \mathrm{P}$ NMR for the reaction of $\mathbf{2 a}$ and $\mathbf{2} \mathbf{b}$ in $\mathrm{CDCl}_{3}(162 \mathrm{MHz})$

Aug20-2020

$\mathrm{LY}-\mathrm{BU}-1$

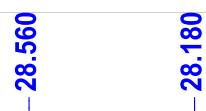

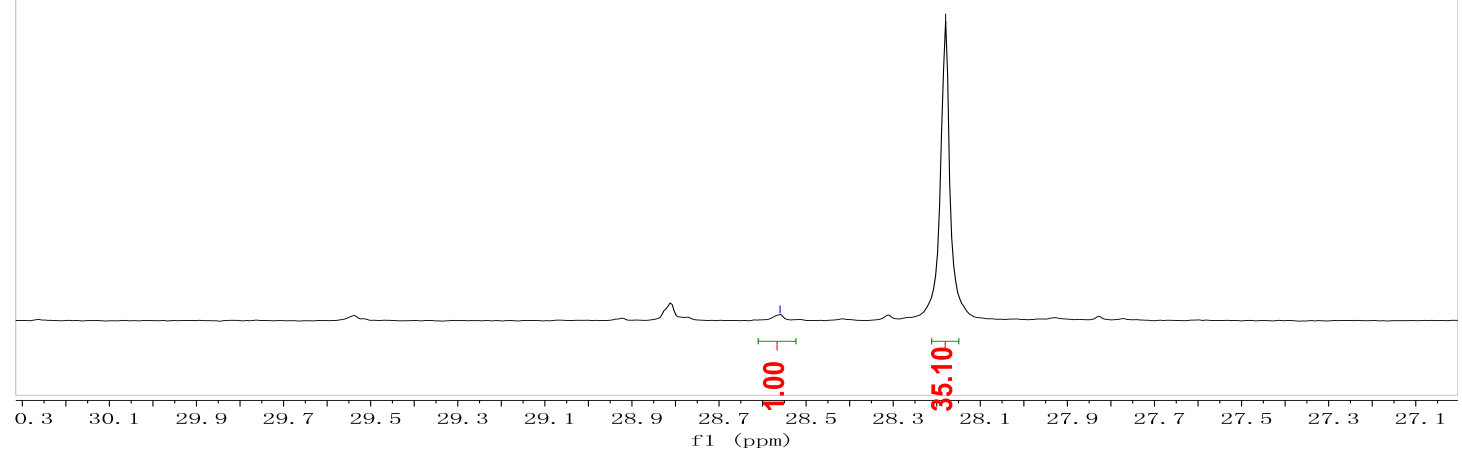


${ }^{1} \mathrm{H}$ NMR for the reaction of $\mathbf{2 a}$ and $\mathbf{2 c}$ in $\mathrm{CDCl}_{3}(400 \mathrm{MHz})$

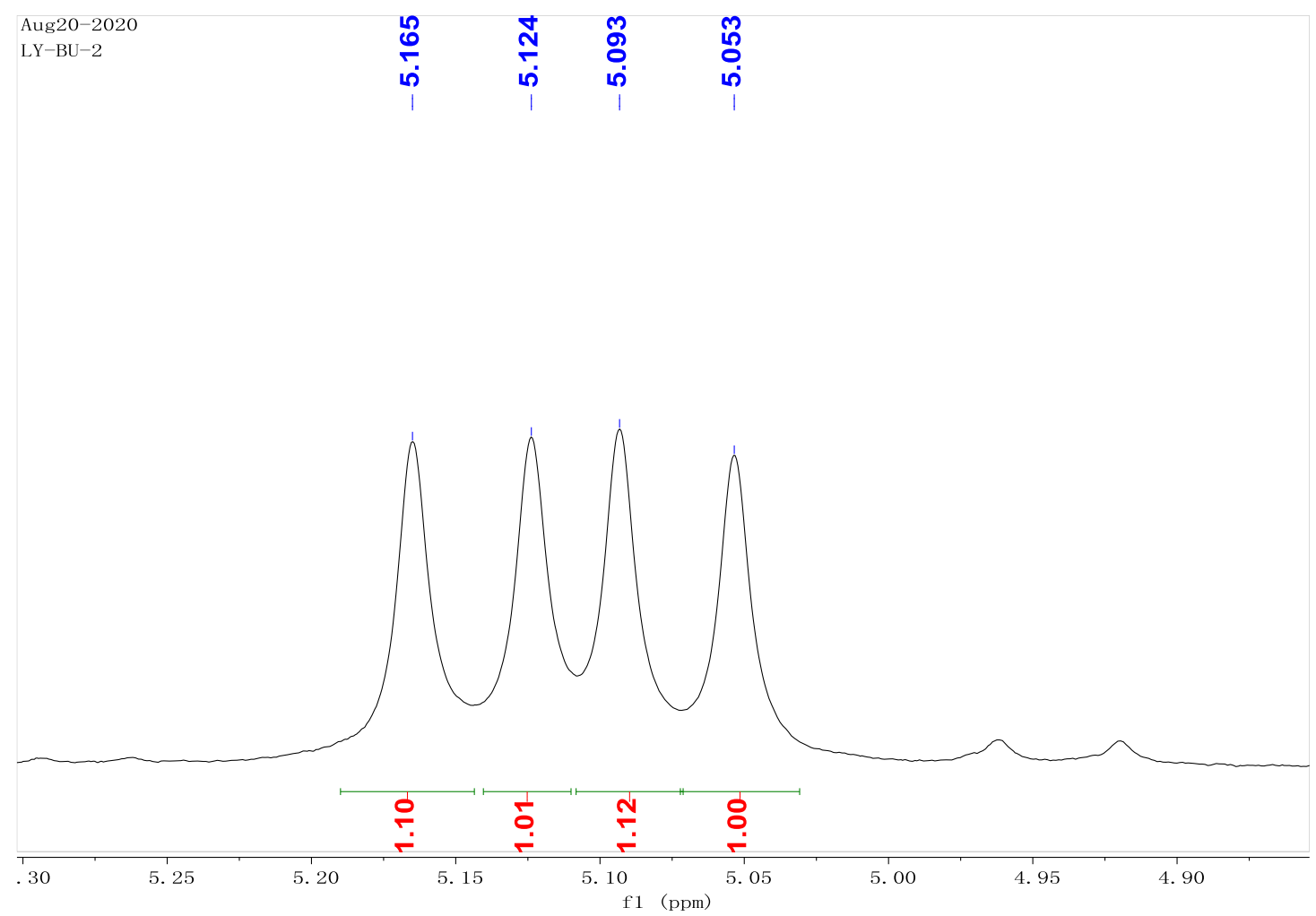

${ }^{31} \mathrm{P}$ NMR for the reaction of $\mathbf{2 a}$ and $\mathbf{2 c}$ in $\mathrm{CDCl}_{3}(162 \mathrm{MHz})$

Aug20-2020 LY-BU-2
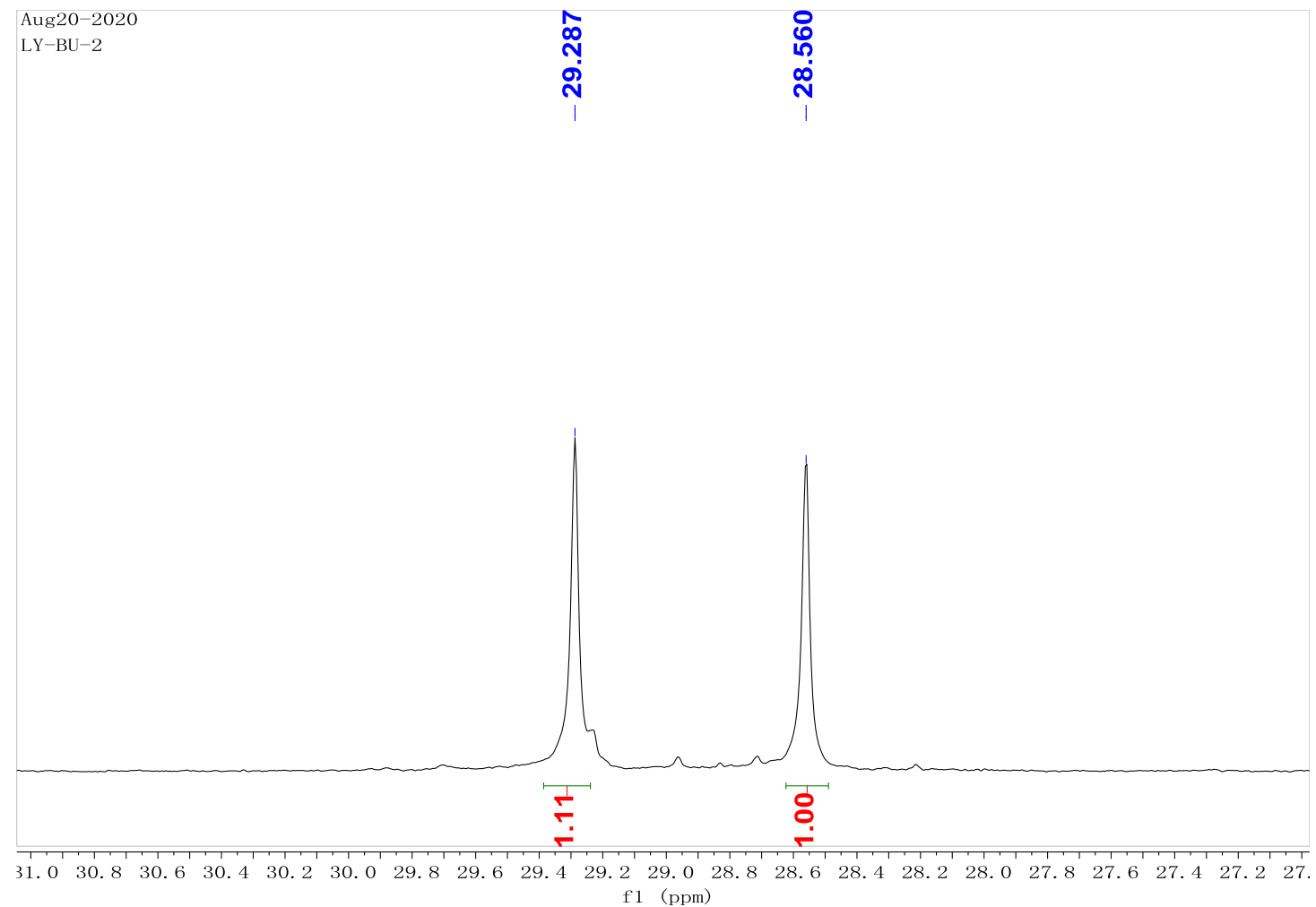
${ }^{1} \mathrm{H}$ NMR for the reaction of $\mathbf{2 a}$ and $\mathbf{2 l}$ in $\mathrm{CDCl}_{3}(400 \mathrm{MHz})$

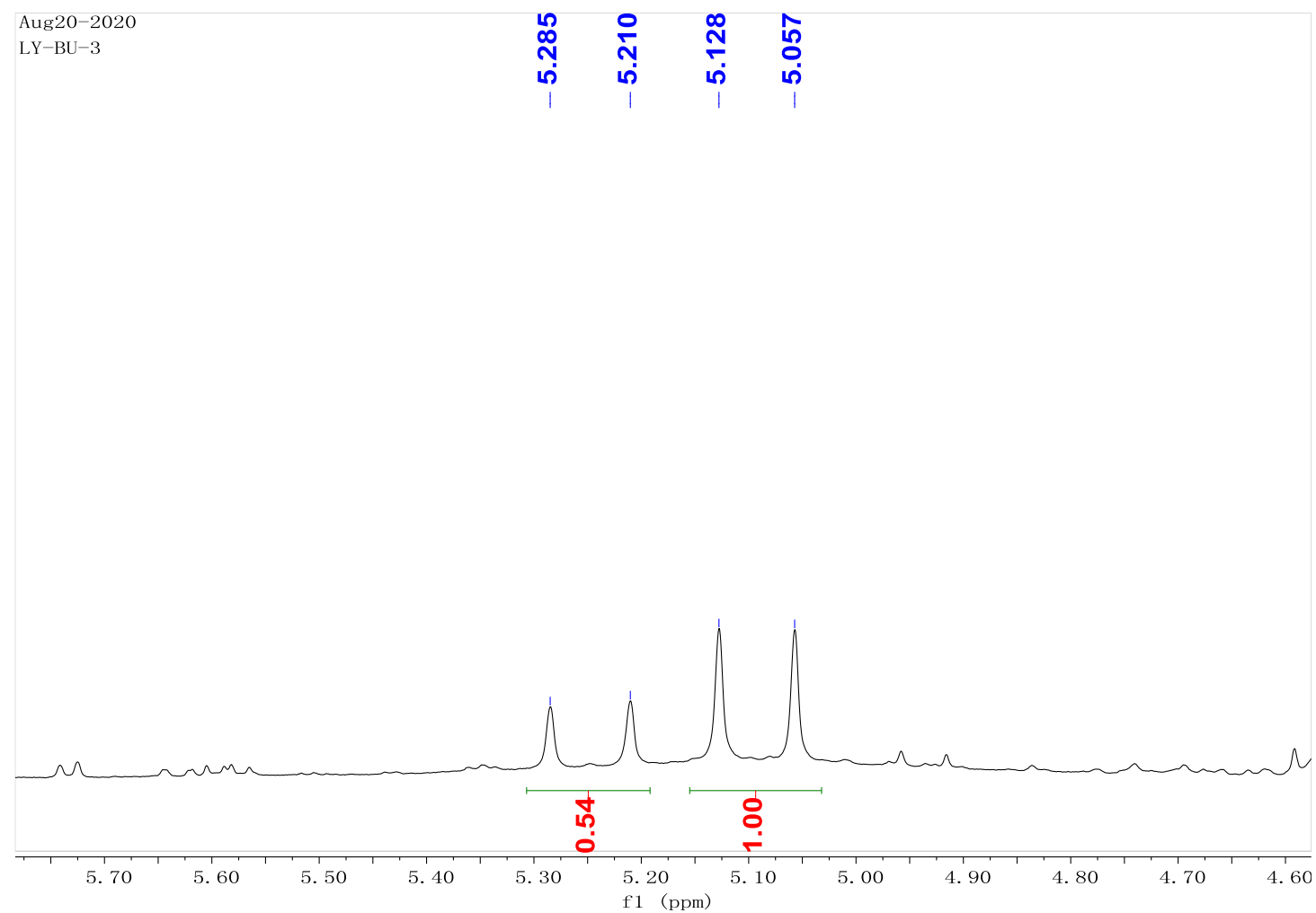

${ }^{31} \mathrm{P}$ NMR for the reaction of $\mathbf{2 a}$ and $\mathbf{2 l}$ in $\mathrm{CDCl}_{3}(162 \mathrm{MHz})$

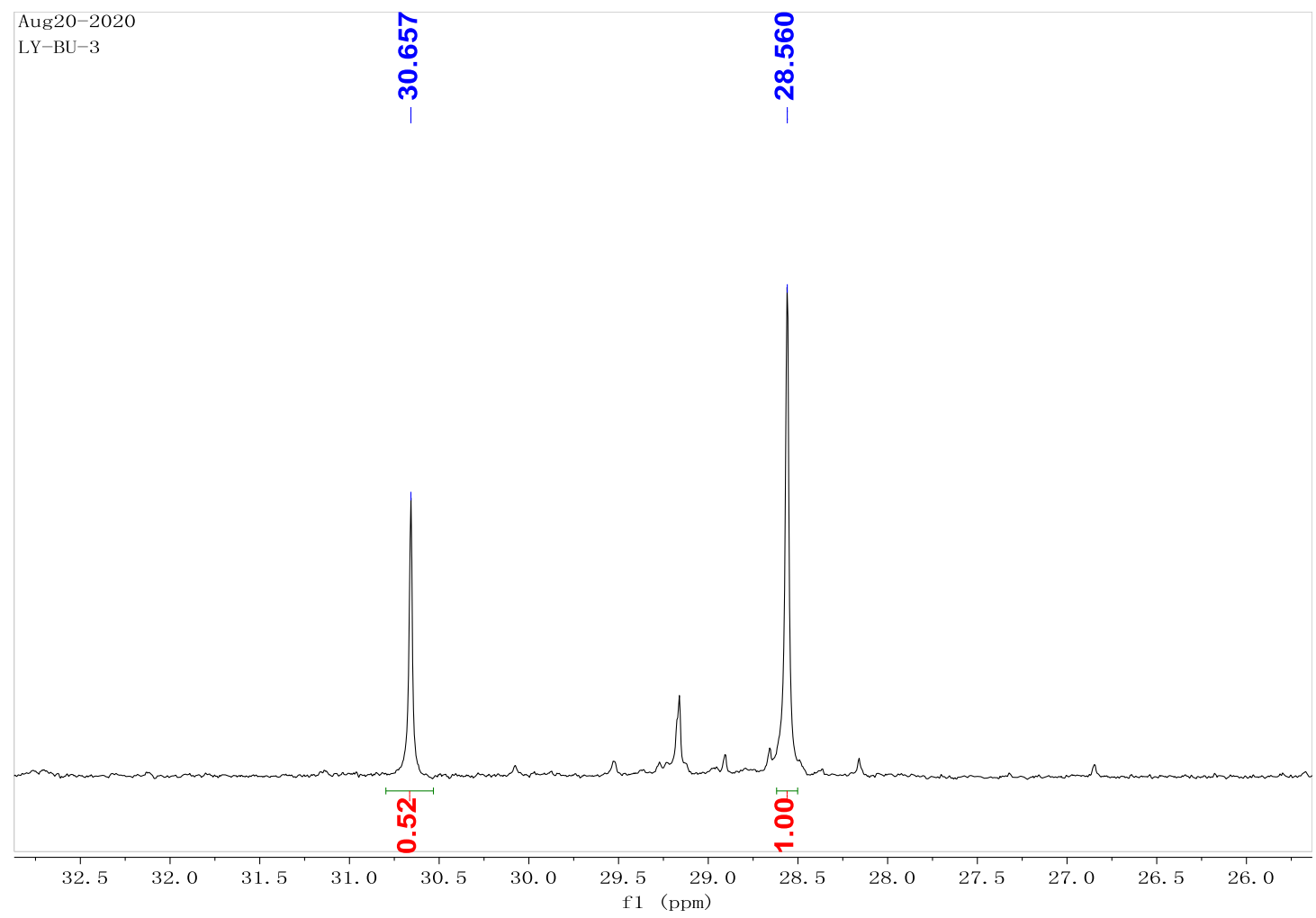


${ }^{1} \mathrm{H}$ NMR for the reaction of $\mathbf{2 a}$ and $\mathbf{2 i}$ in $\mathrm{CDCl}_{3}(400 \mathrm{MHz})$

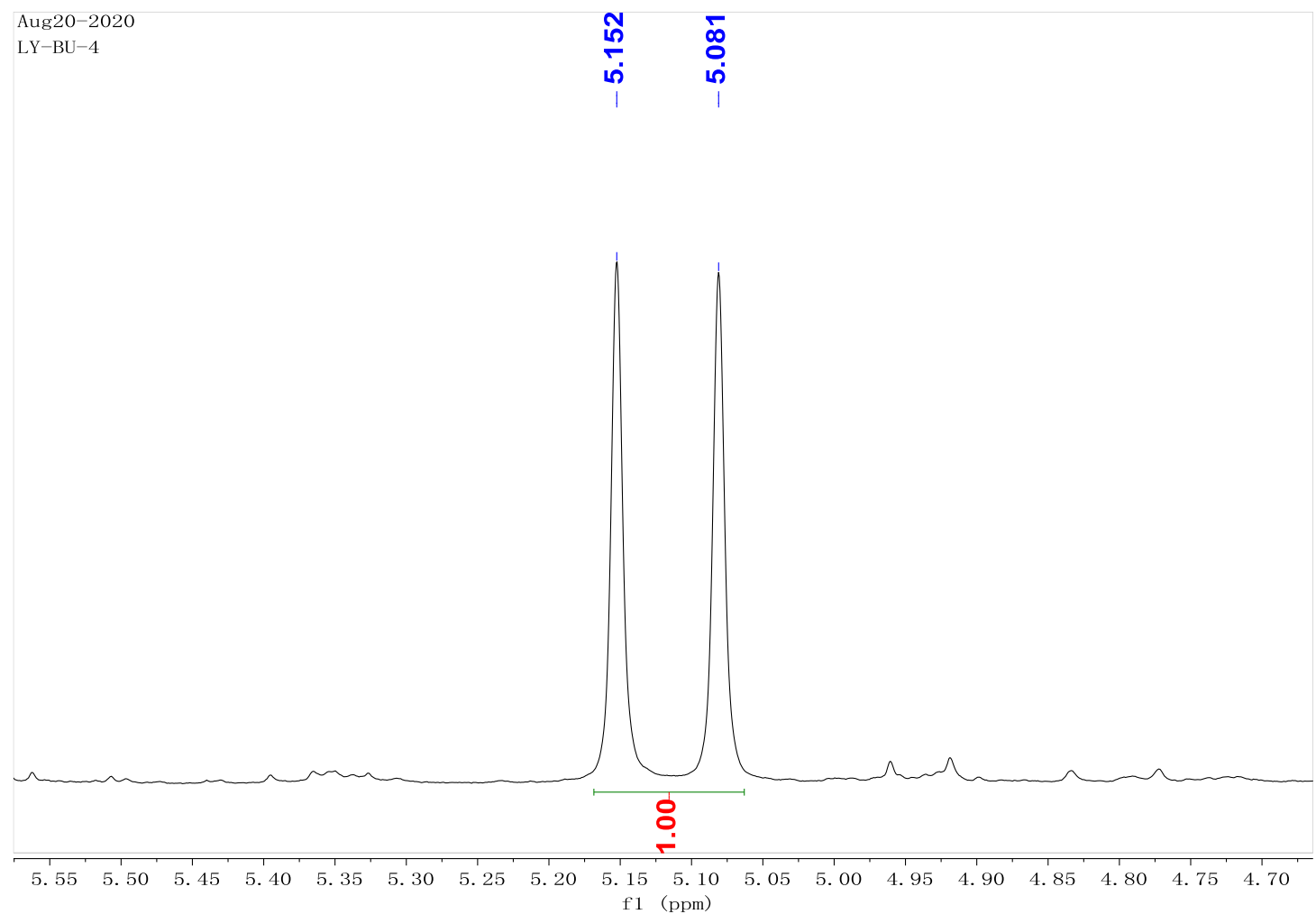

${ }^{31} \mathrm{P}$ NMR for the reaction of $\mathbf{2 a}$ and $\mathbf{2} \mathbf{i}$ in $\mathrm{CDCl}_{3}(162 \mathrm{MHz})$

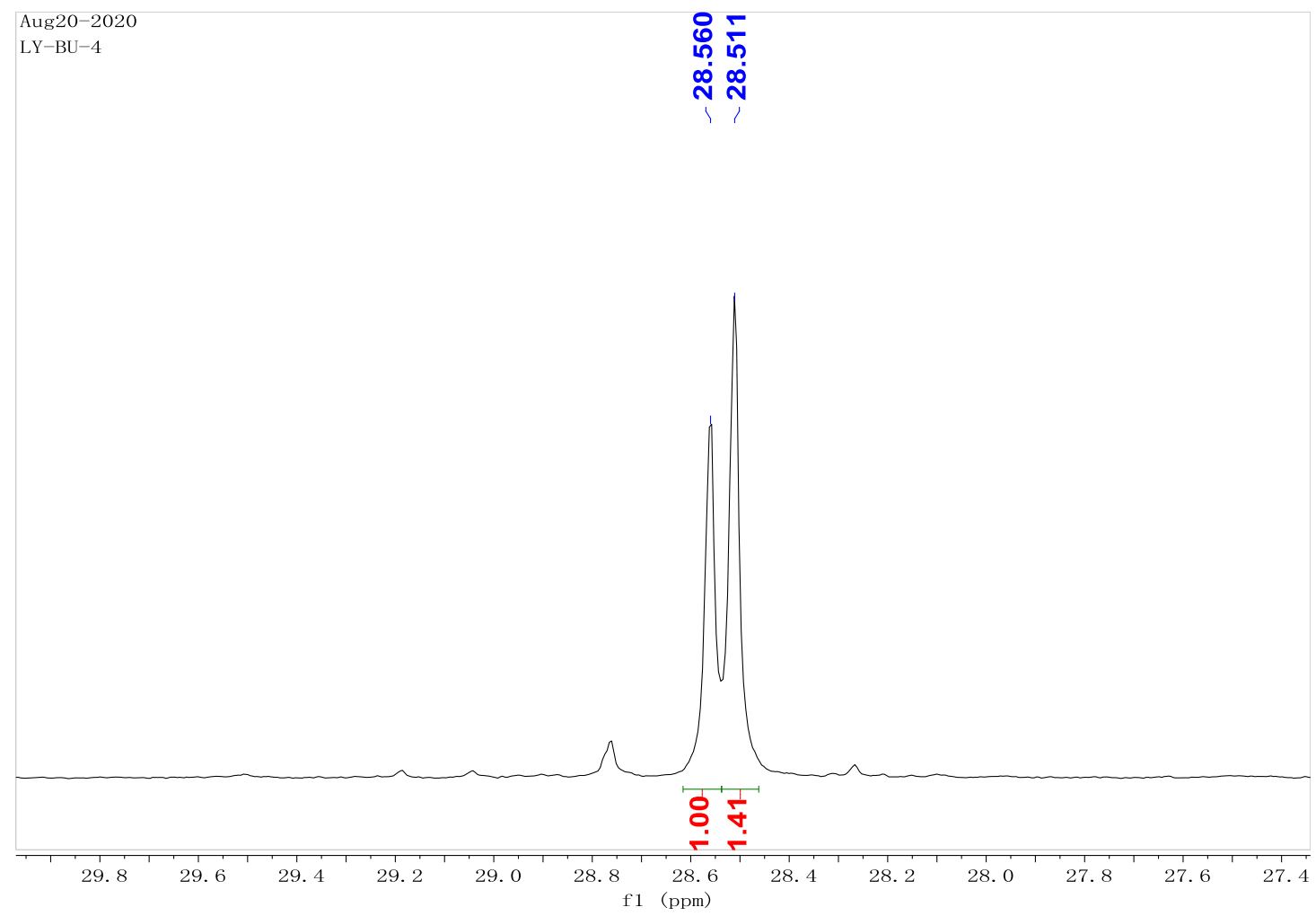


${ }^{1} \mathrm{H}$ NMR for the reaction of $\mathbf{2 a}$ and $\mathbf{2} \mathbf{j}$ in $\mathrm{CDCl}_{3}(400 \mathrm{MHz})$

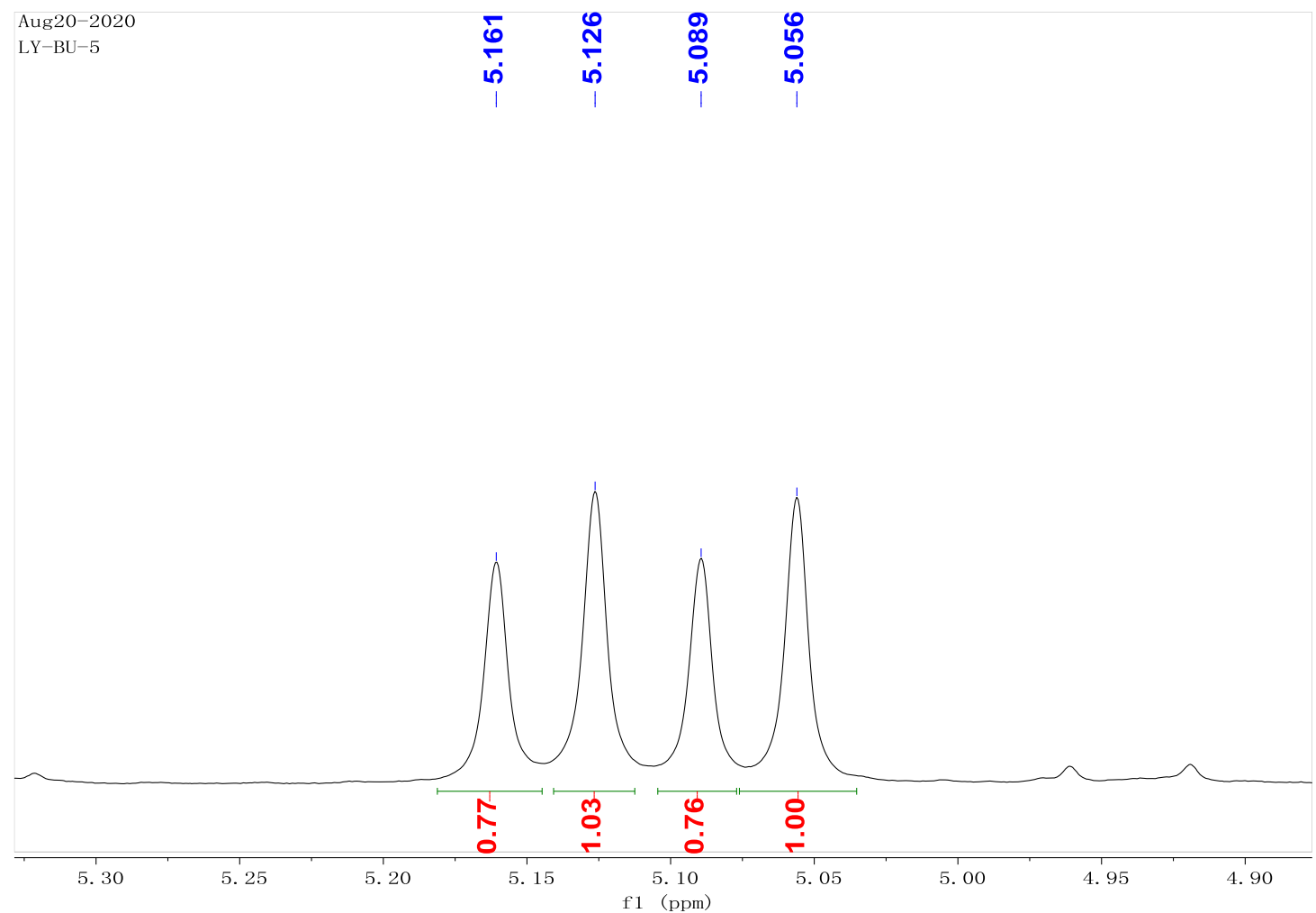

${ }^{31} \mathrm{P}$ NMR for the reaction of $\mathbf{2 a}$ and $\mathbf{2} \mathbf{j}$ in $\mathrm{CDCl}_{3}(162 \mathrm{MHz})$

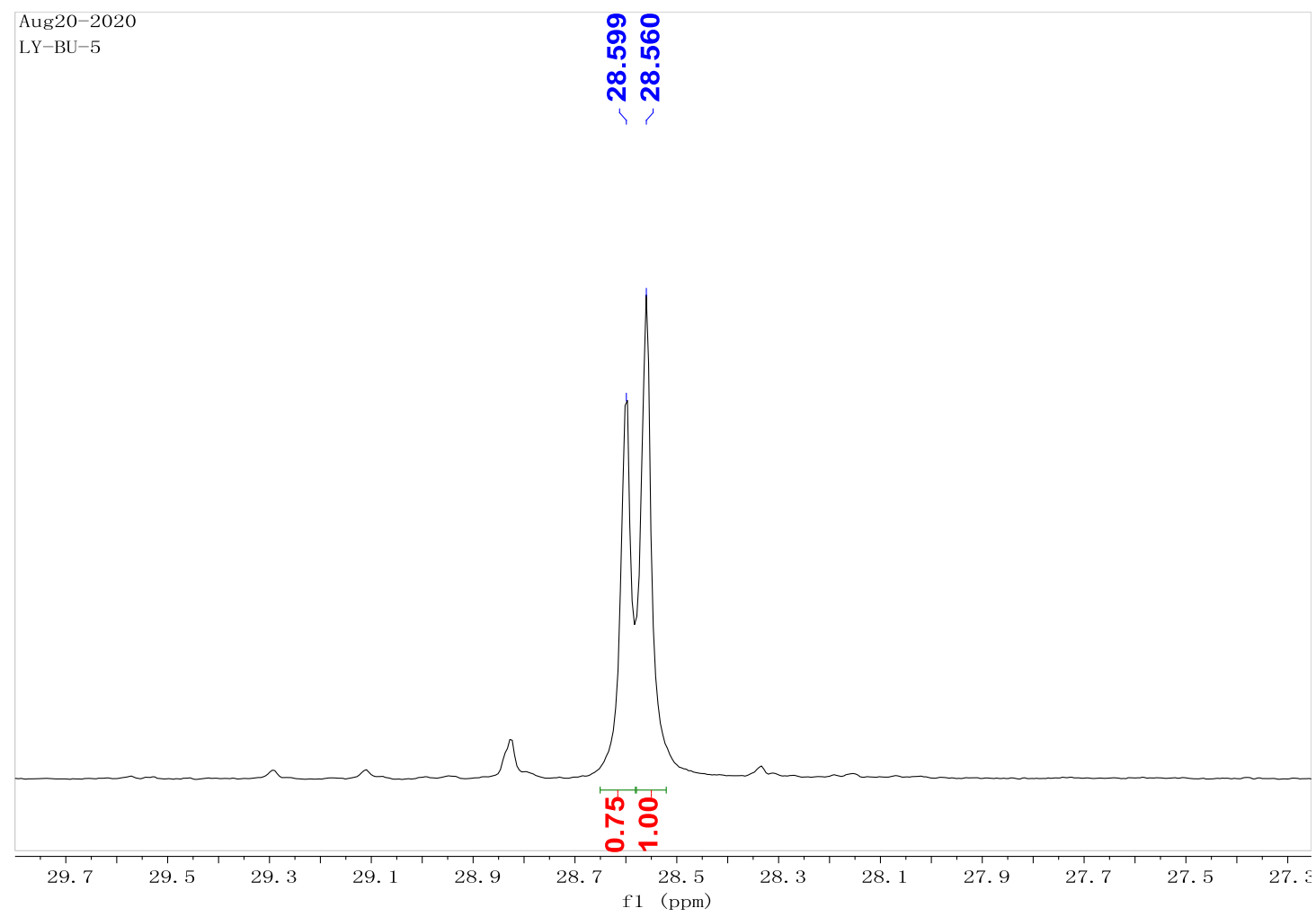


${ }^{1} \mathrm{H}$ NMR for the reaction of $\mathbf{2 a}$ and $\mathbf{2 k}$ in $\mathrm{CDCl}_{3}(400 \mathrm{MHz})$

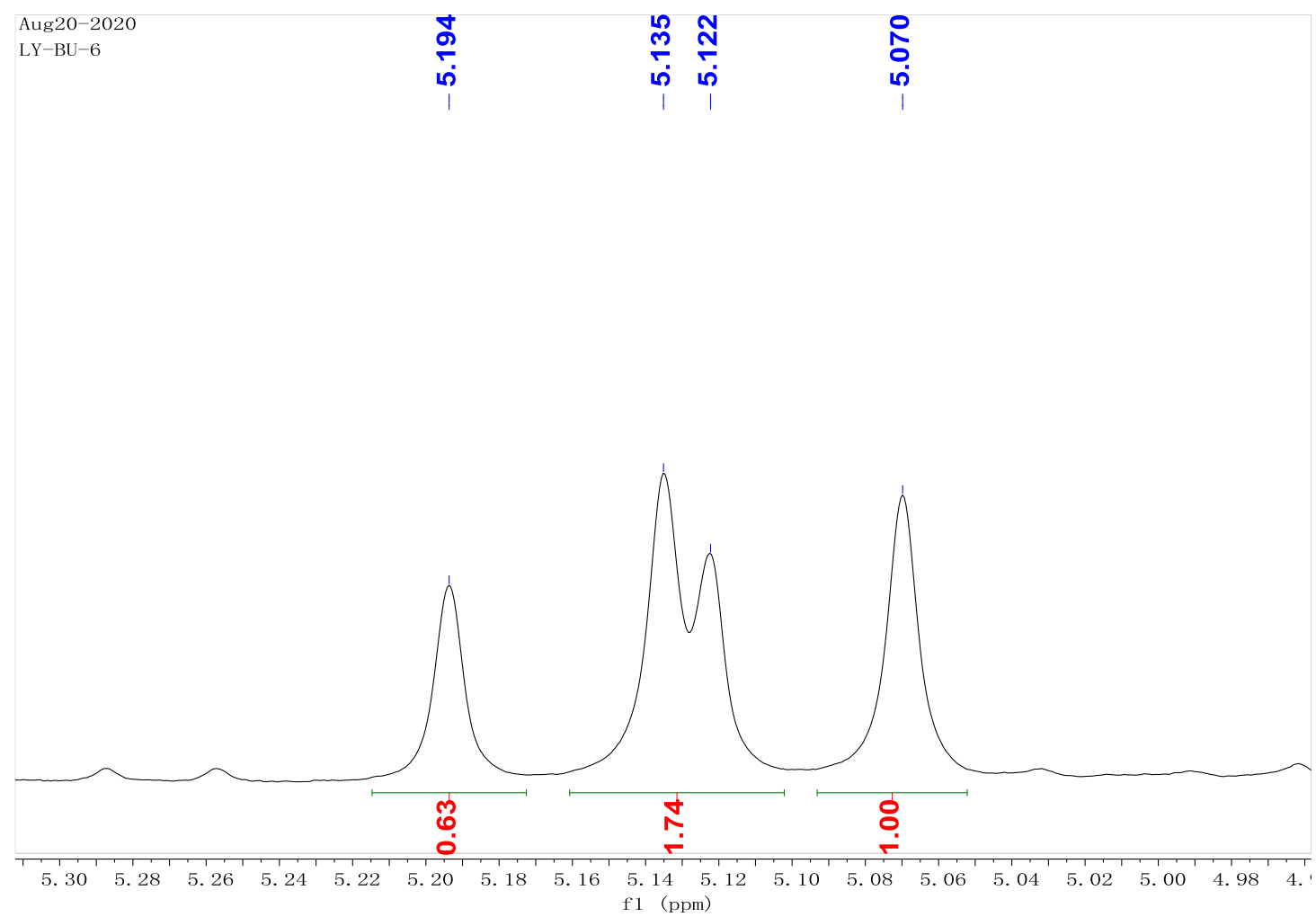

${ }^{31} \mathrm{P}$ NMR for the reaction of $\mathbf{2 a}$ and $\mathbf{2 k}$ in $\mathrm{CDCl}_{3}(162 \mathrm{MHz})$

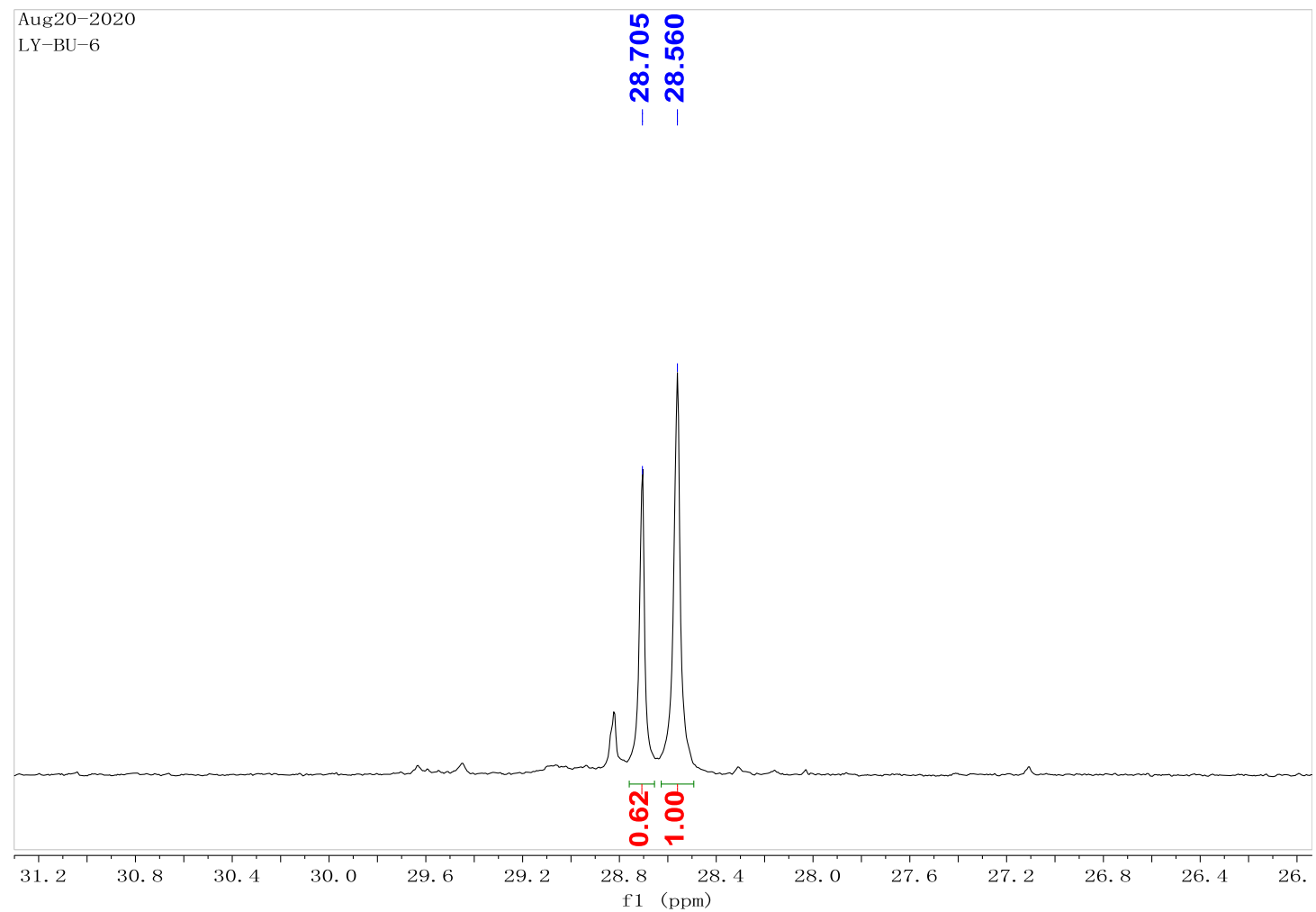




\section{References}

1. Regitz, M.; Eckes, H. Chem. Ber. 1980, 113, 3303-3312.

2. Jin, C.; Liang, Y.-J.; He, H.; Fu, L. Phosphorus Sulfur Silicon Relat. Elem. 2011, 186, 2096-2103.

3. He, X.-L.; Zhao, H.-R.; Song, X.; Jiang, B.; Du, W.; Chen, Y.-C. ACS Catal. 2019, 9, 4374-4381.

4. Heydt, H. Sci. Synth. 2004, 27, 843-935.

5. Zhou, Y.; Zhang, Y.; Wang, J. Org. Biomol. Chem. 2016, 14, 10444-10453.

6. Ren, Y.-Y.; Wang, Y.-Q.; Liu, S.; Pan, K. Chem CatChem. 2014, 6, 2985-2992.

7. Fakhraian, H.; Nafary, Y.; Yarahmadi, A.; Hadj-Ghanbary, H. J. Heterocycl.

Chem. 2008, 45, 1469-1472.

8. Wardrop, A. W. H.; Sainsbury, G. L.; Harrison, J. M.; Inch, T. D. J. Chem. Soc., Perkin Trans. 1. 1976, 1279-1285.

9. Ghafarzadeh, M.; Moghadam, E. S.; Faraji, F. J. Heterocycl. Chem. 2013, 50, $754-757$.

10. Li, B.-Y.; Lin, Y.; Du, D.-M. J. Org. Chem. 2019, 84, 11752-11762.

11. Gutch, P. K.; Acharya, J. Heterocycl. Commun. 2007, 13, 393-396.

12. Lin, Y.-C.; Li, N.-C.; Cherng, Y.-J. J. Heterocycl. Chem. 2014, 51, 808-814.

13. Fujii, T.; Hao, W.; Yoshimura, T. Heteroat. Chem. 2004, 15, 246-250.

14. Deng, Z.-F.; Huang, B.; Xu, H.; Shi, F.; Wang, Y.-Q. Asian J. Org. Chem. 2017, 6, $1460-1469$.

15. Sowinski, F. A.; Yale, H. L. Dihydrodibenzothiazepines. US3188320, 1965.

16. Qi, H. Z.; Li, X. Y.; Xu, J. X. Org. Biomol. Chem. 2011, 9, 2702-2714.

17. Guo, K.; Yu, C.-B.; Li, W.; Zhou, Y. G.; Zhang, X. M. Chem. Commun. 2011, 47, $4845-7847$. 


\section{Copies of ${ }^{1} \mathrm{H},{ }^{13} \mathrm{C},{ }^{19} \mathrm{~F}$, and ${ }^{31} \mathrm{P}$ NMR Spectra}

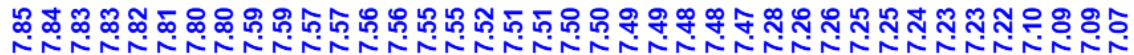

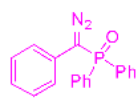

${ }^{1} \mathrm{H}(400 \mathrm{MHz}), \mathrm{CDCl}_{3}$

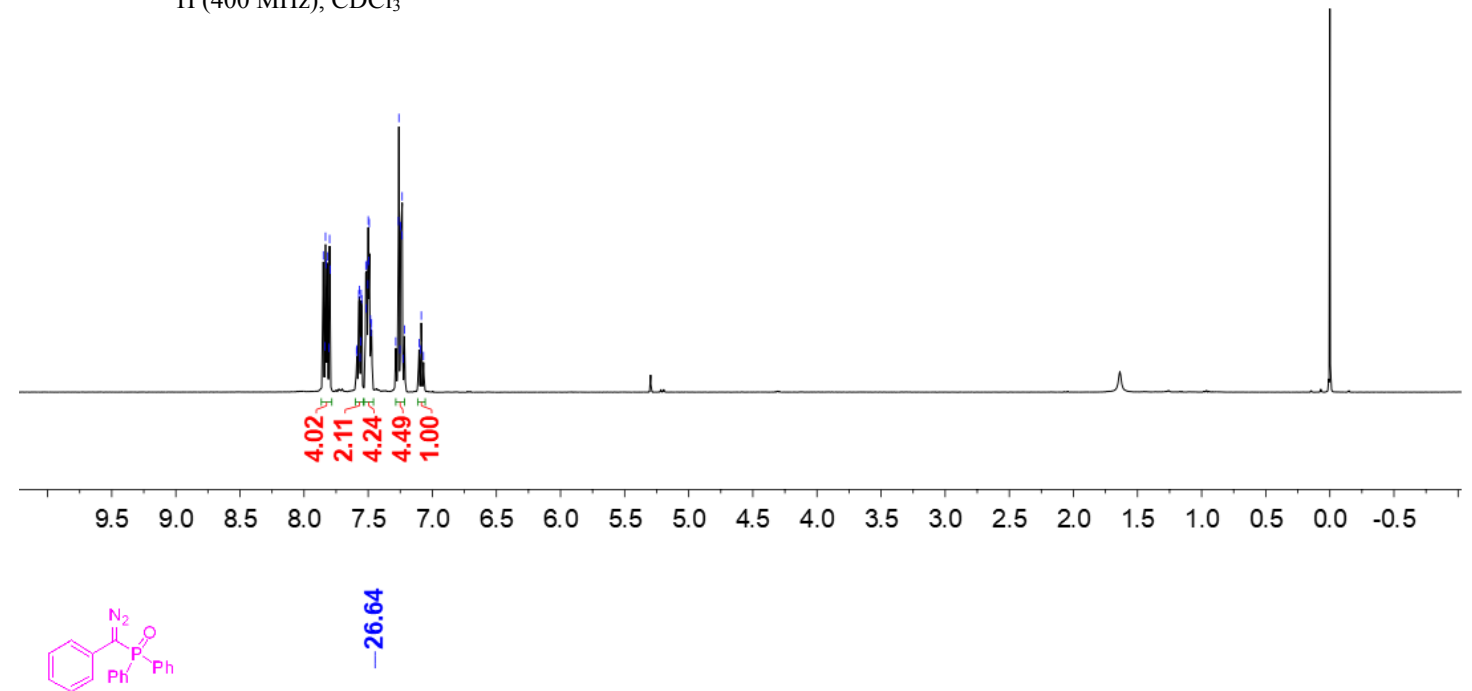

${ }^{31} \mathrm{P}(162 \mathrm{MHz}), \mathrm{CDCl}_{3}$

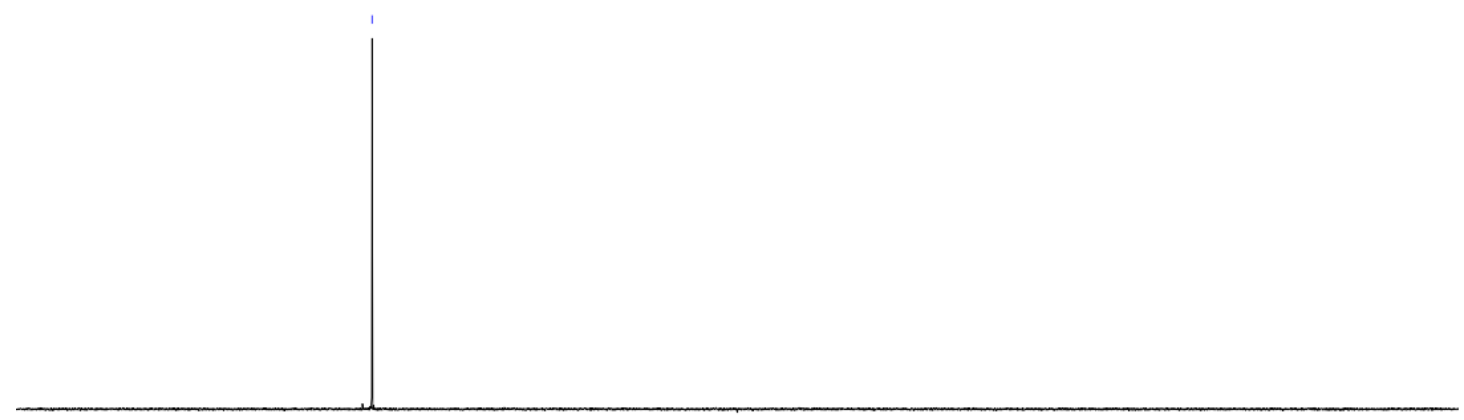

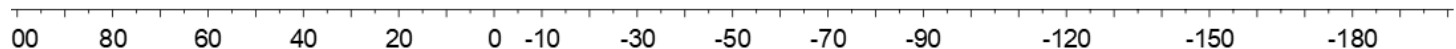




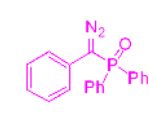

${ }^{13} \mathrm{C}(101 \mathrm{MHz}), \mathrm{CDCl}_{3}$

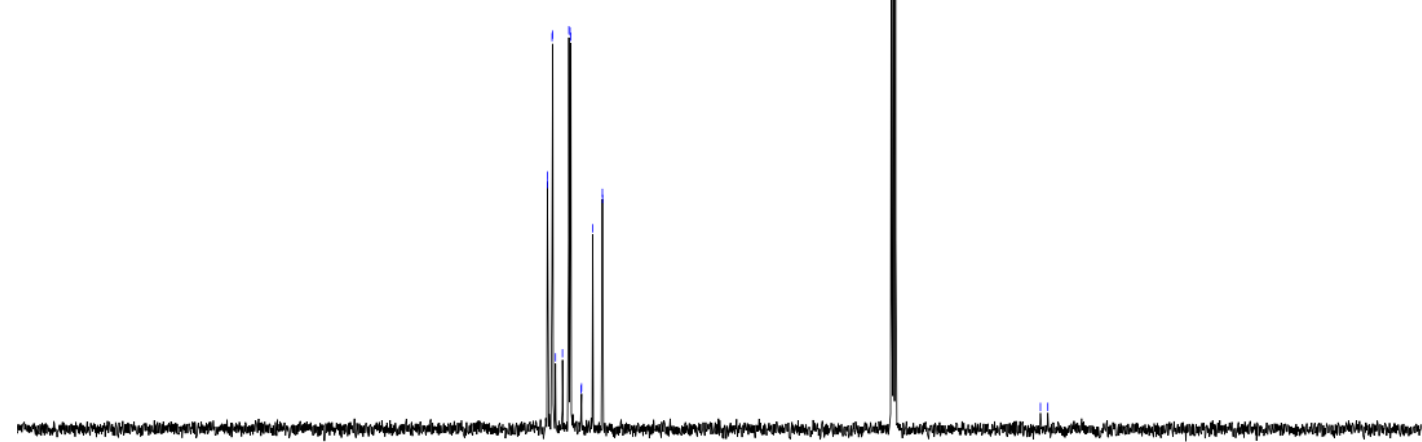

$\begin{array}{lllllllllllllllll}210 & 190 & 170 & 150 & 130 & 110 & 90 & 80 & 70 & 60 & 50 & 40 & 30 & 20 & 10 & 0\end{array}$

${ }^{1} \mathrm{H}(400 \mathrm{MHz}),{ }^{31} \mathrm{P}(162 \mathrm{MHz})$ and ${ }^{13} \mathrm{C}(101 \mathrm{MHz})$ spectra of compound 1a

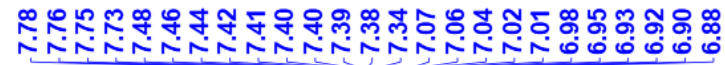

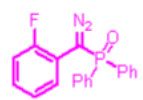

${ }^{1} \mathrm{H}(400 \mathrm{MHz}), \mathrm{CDCl}_{3}$

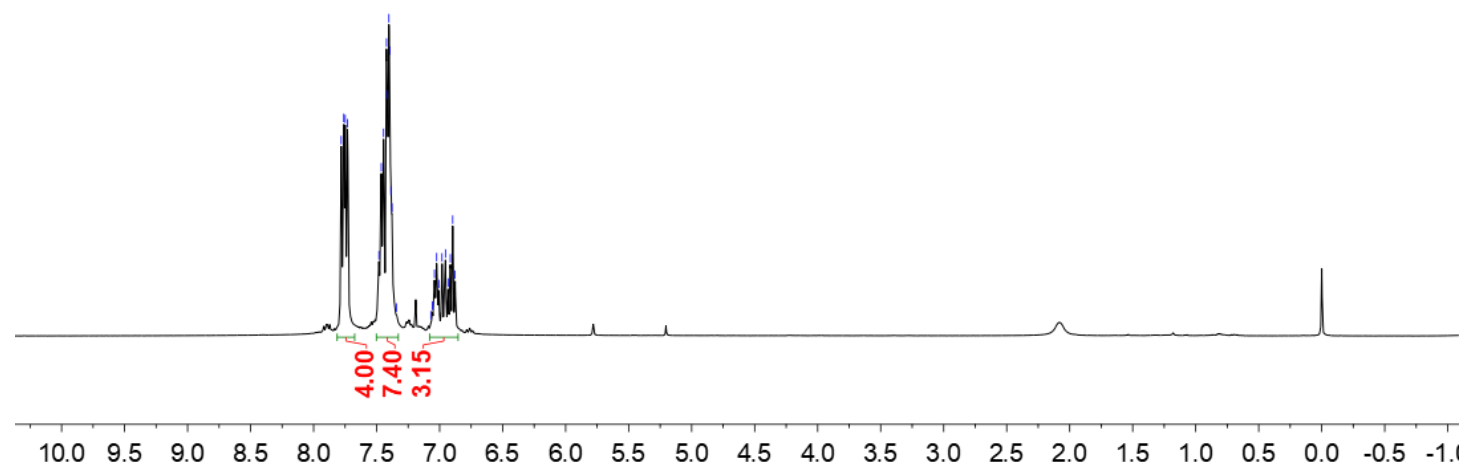




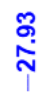

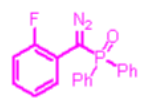

${ }^{31} \mathrm{P}(162 \mathrm{MHz}), \mathrm{CDCl}_{3}$

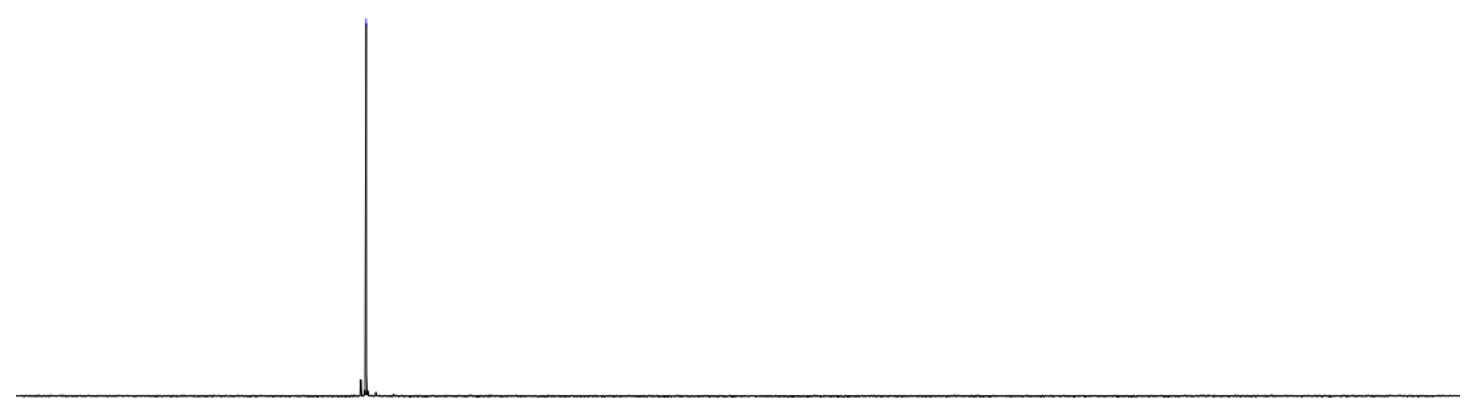

\begin{tabular}{|c|c|c|c|c|c|c|c|c|c|c|c|c|c|c|}
\hline 00 & 80 & 60 & 40 & 20 & 0 & -20 & -40 & -60 & -80 & -100 & -120 & -140 & -160 & -180 \\
\hline
\end{tabular}

\section{$\frac{5}{i}$}

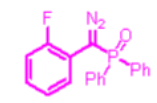

${ }^{19} \mathrm{~F}(376 \mathrm{MHz}), \mathrm{CDCl}_{3}$

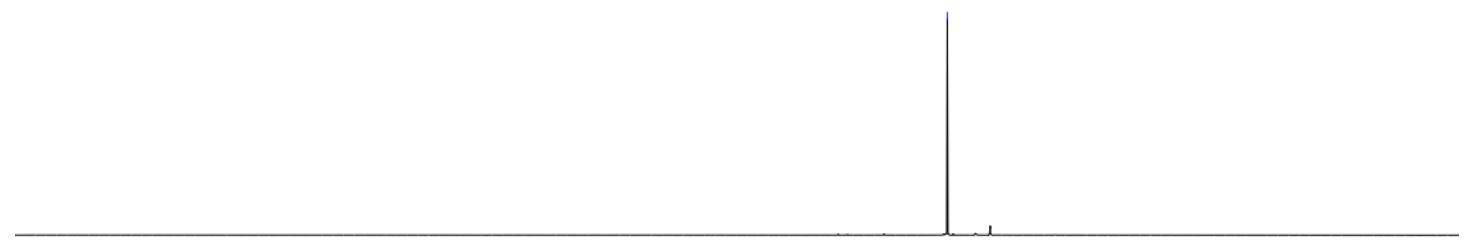

$\begin{array}{lllllllllllllllllll}-55 & -60 & -65 & -70 & -75 & -80 & -85 & -90 & -95 & -100 & -105 & -110 & -115 & -120 & -125 & -130 & -135 & -140 & -145\end{array}$ 


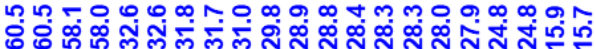

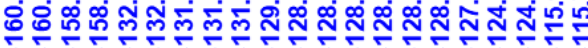

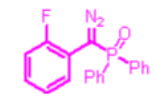

${ }^{13} \mathrm{C}(101 \mathrm{MHz}), \mathrm{CDCl}_{3}$

$\begin{array}{llllllllllllllllllllll}210 & 200 & 190 & 180 & 170 & 160 & 150 & 140 & 130 & 120 & 110 & 100 & 90 & 80 & 70 & 60 & 50 & 40 & 30 & 20 & 10 & 0\end{array}$

${ }^{1} \mathrm{H}(400 \mathrm{MHz}),{ }^{31} \mathrm{P}(162 \mathrm{MHz}),{ }^{19} \mathrm{~F}$ NMR $(376 \mathrm{MHz})$ and ${ }^{13} \mathrm{C}(101 \mathrm{MHz})$ spectra of compound $\mathbf{1 b}$

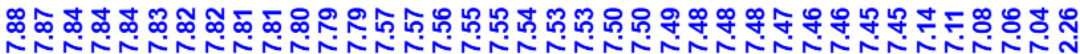

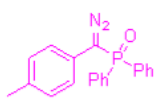

${ }^{1} \mathrm{H}(400 \mathrm{MHz}), \mathrm{CDCl}_{3}$

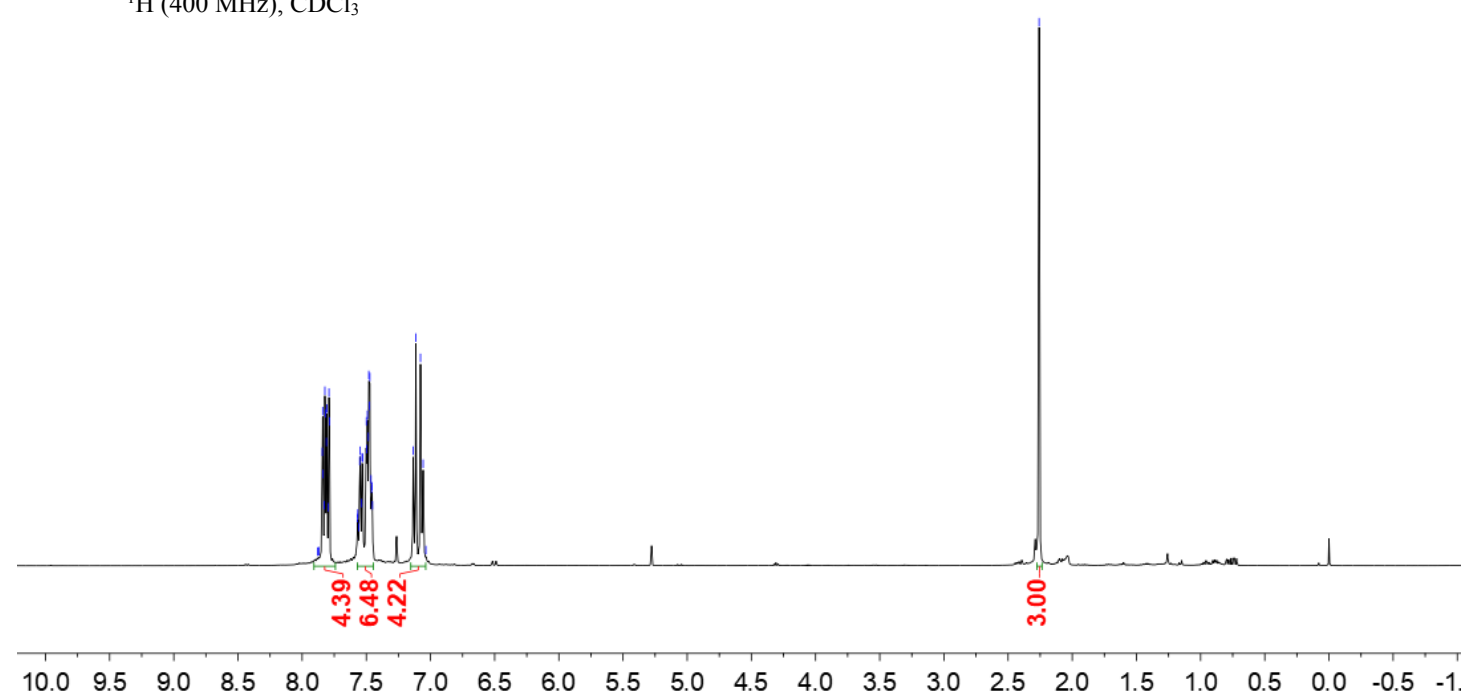




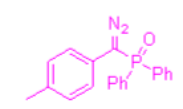

${ }^{31} \mathrm{P}(162 \mathrm{MHz}), \mathrm{CDCl}_{3}$
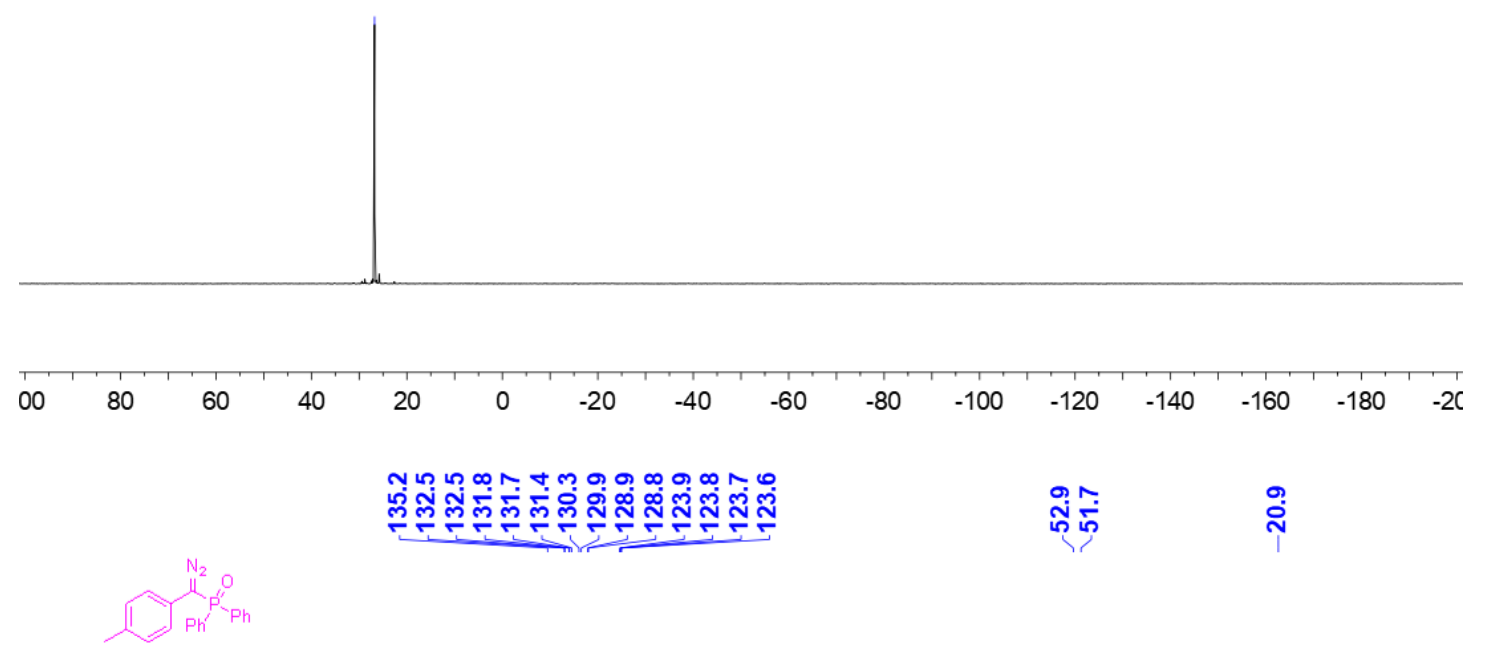

${ }^{13} \mathrm{C}(101 \mathrm{MHz}), \mathrm{CDCl}_{3}$

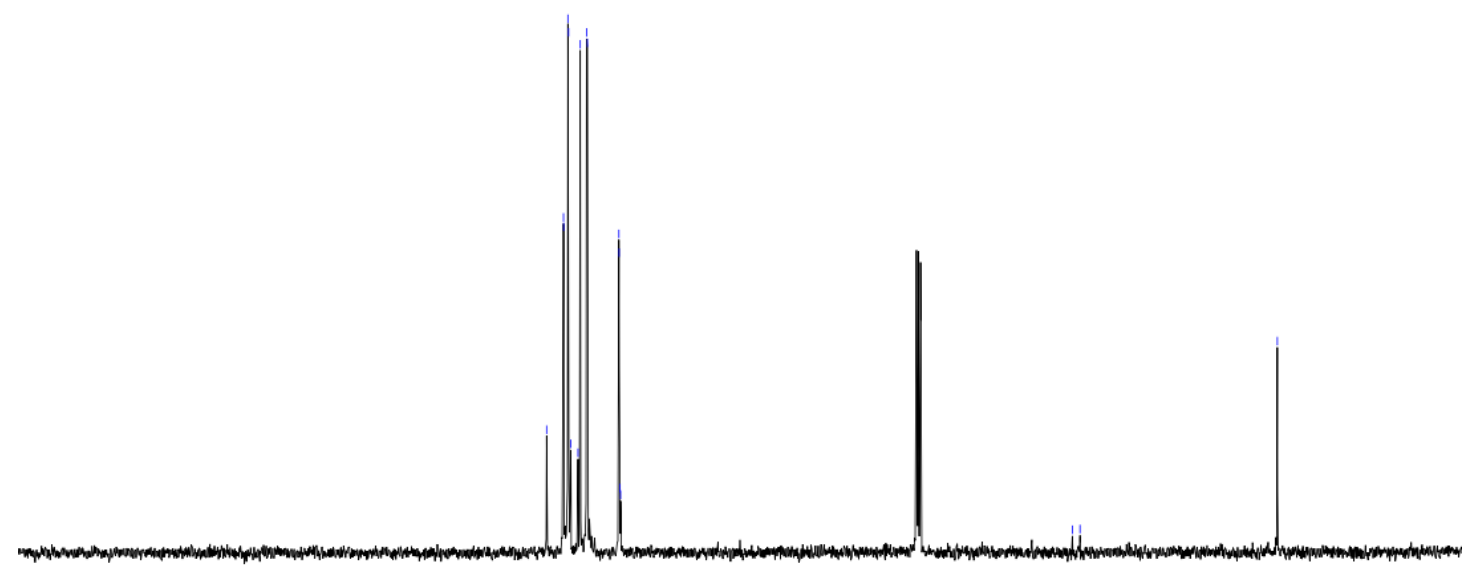

$\begin{array}{llllllllllllllllllllll}210 & 200 & 190 & 180 & 170 & 160 & 150 & 140 & 130 & 120 & 110 & 100 & 90 & 80 & 70 & 60 & 50 & 40 & 30 & 20 & 10 & 0\end{array}$

${ }^{1} \mathrm{H}(400 \mathrm{MHz}),{ }^{31} \mathrm{P}(162 \mathrm{MHz})$ and ${ }^{13} \mathrm{C}(101 \mathrm{MHz})$ spectra of compound 1c 


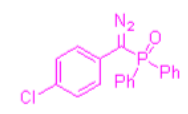

${ }^{1} \mathrm{H}(400 \mathrm{MHz}), \mathrm{CDCl}_{3}$
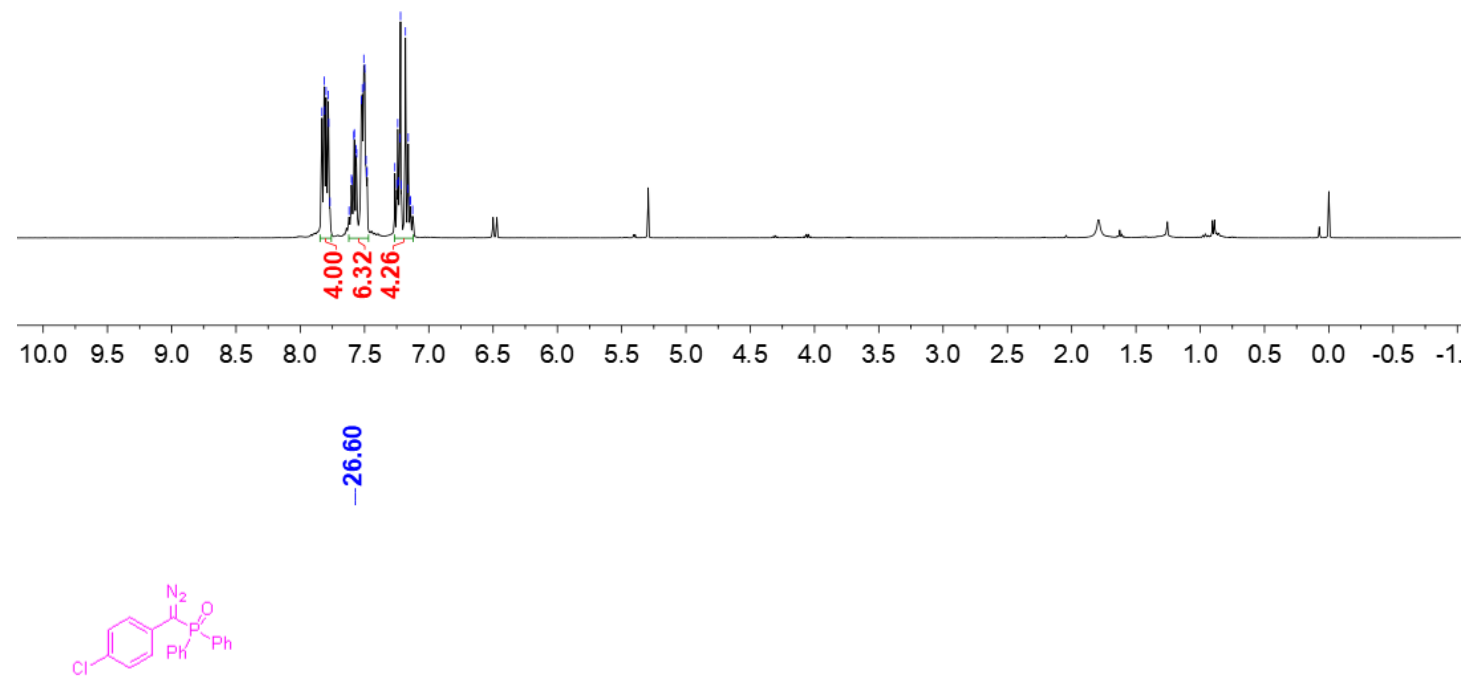

${ }^{31} \mathrm{P}(162 \mathrm{MHz}), \mathrm{CDCl}_{3}$

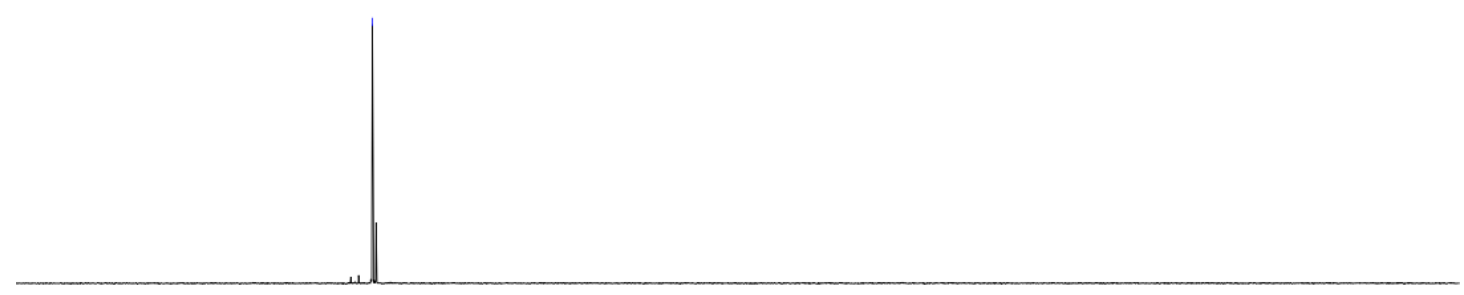

$\begin{array}{llllllllllllllll}00 & 80 & 60 & 40 & 20 & 0 & -20 & -40 & -60 & -80 & -100 & -120 & -140 & -160 & -180 & -2 C\end{array}$ 


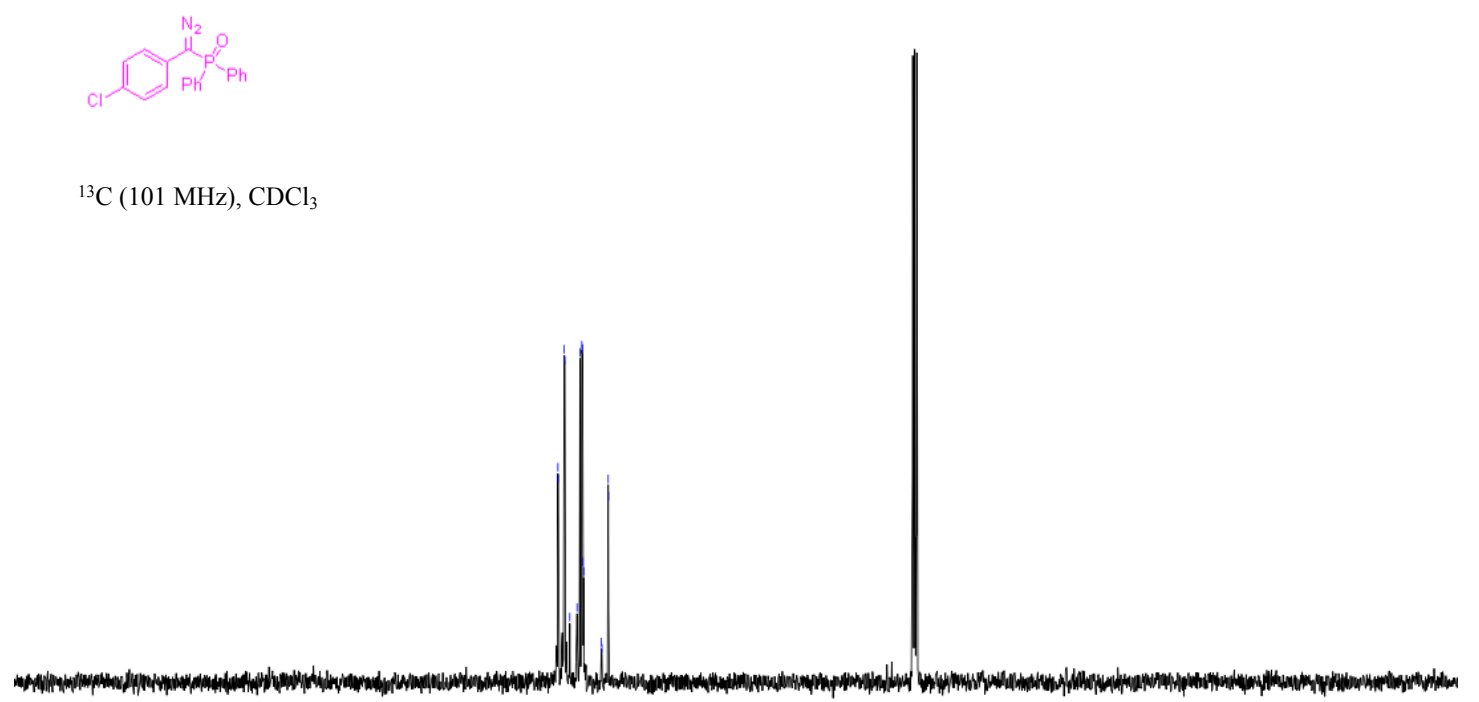

$\begin{array}{llllllllllllllllllllll}210 & 200 & 190 & 180 & 170 & 160 & 150 & 140 & 130 & 120 & 110 & 100 & 90 & 80 & 70 & 60 & 50 & 40 & 30 & 20 & 10 & 0\end{array}$

${ }^{1} \mathrm{H}(400 \mathrm{MHz}),{ }^{31} \mathrm{P}(162 \mathrm{MHz})$ and ${ }^{13} \mathrm{C}(101 \mathrm{MHz})$ spectra of compound $\mathbf{1 d}$

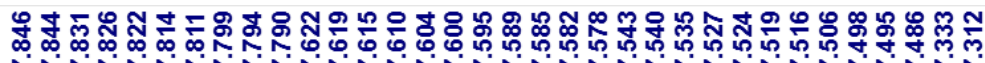

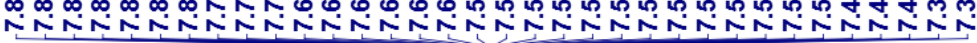

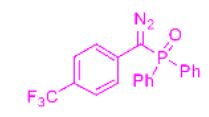

${ }^{1} \mathrm{H}(400 \mathrm{MHz}), \mathrm{CDCl}_{3}$
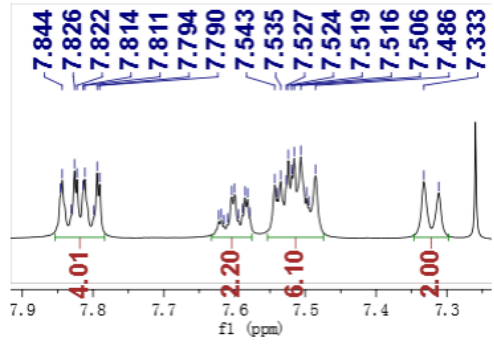

두융응

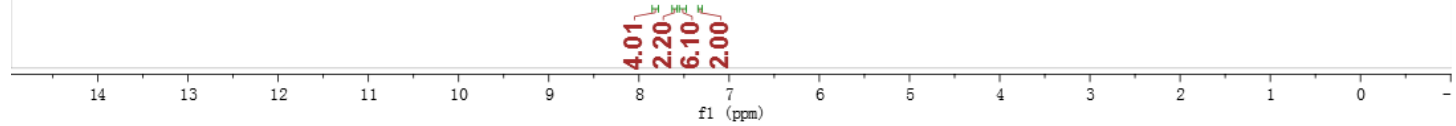




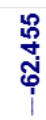

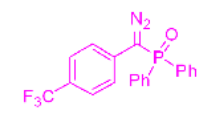

${ }^{19} \mathrm{~F}(376 \mathrm{MHz}), \mathrm{CDCl}_{3}$
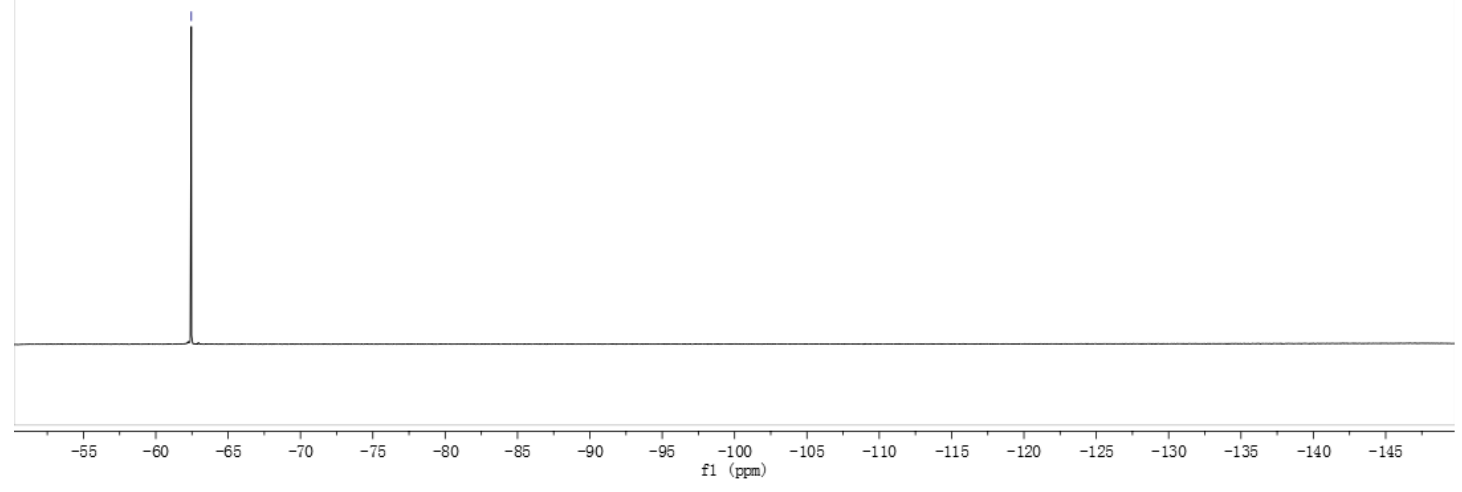

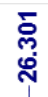

管

${ }^{31} \mathrm{P}(162 \mathrm{MHz}), \mathrm{CDCl}_{3}$

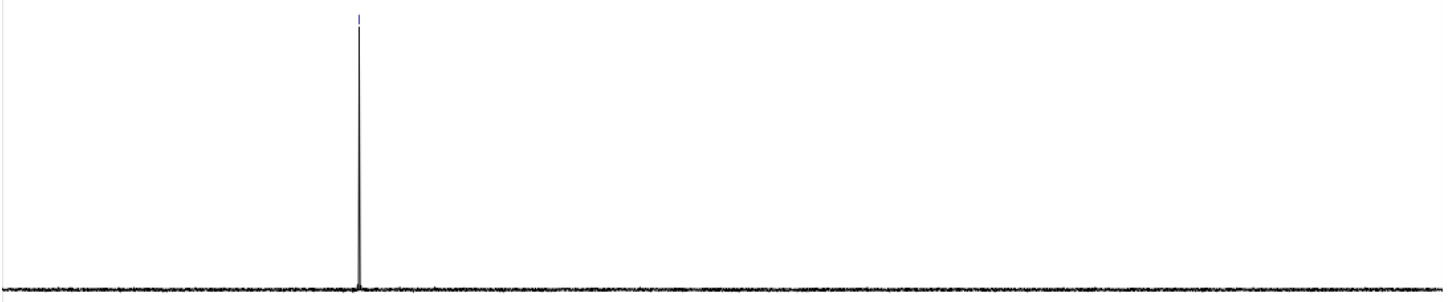

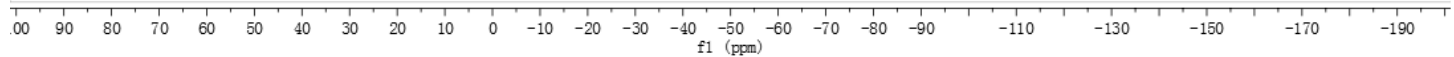




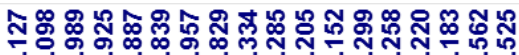

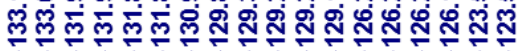

${ }^{13} \mathrm{C}(101 \mathrm{MHz}), \mathrm{CDCl}_{3}$
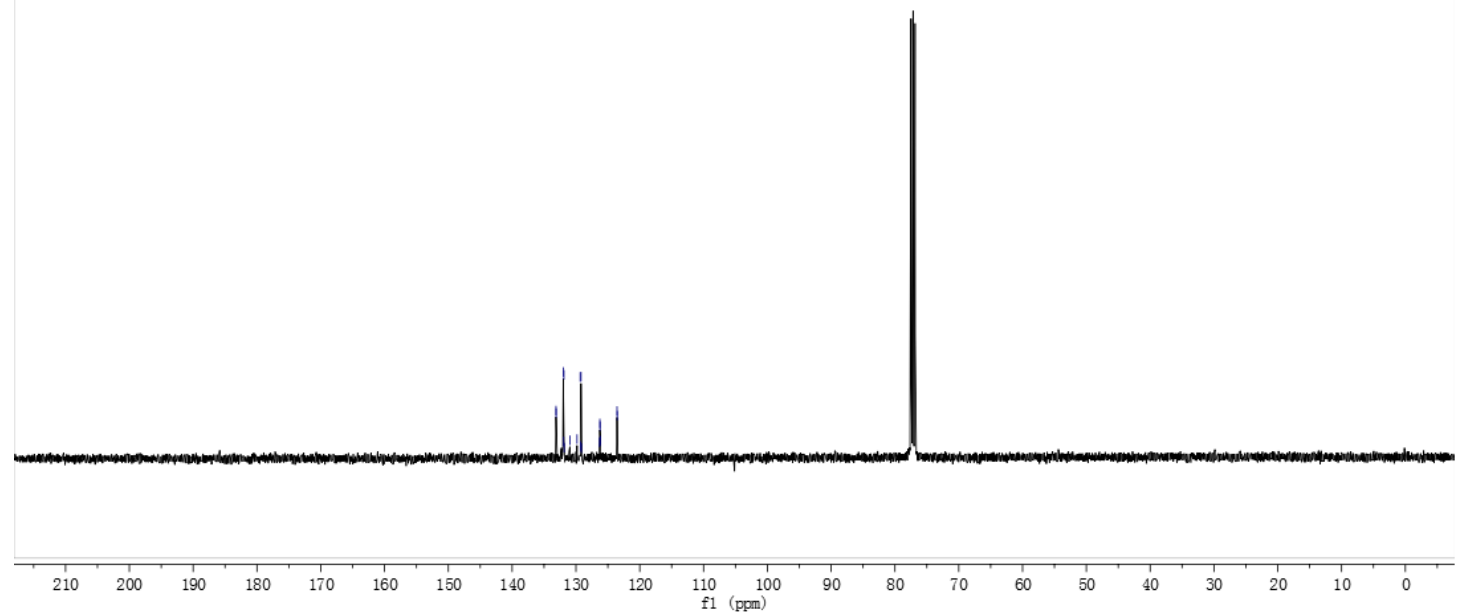

${ }^{1} \mathrm{H}(400 \mathrm{MHz}),{ }^{19} \mathrm{~F}(376 \mathrm{MHz}){ }^{31} \mathrm{P}(162 \mathrm{MHz})$ and ${ }^{13} \mathrm{C}(101 \mathrm{MHz})$ spectra of compound 1e

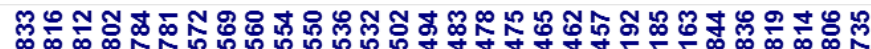

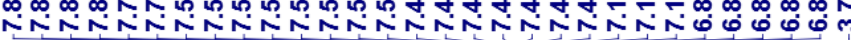

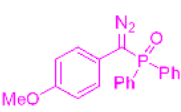

${ }^{1} \mathrm{H}(400 \mathrm{MHz}), \mathrm{CDCl}_{3}$
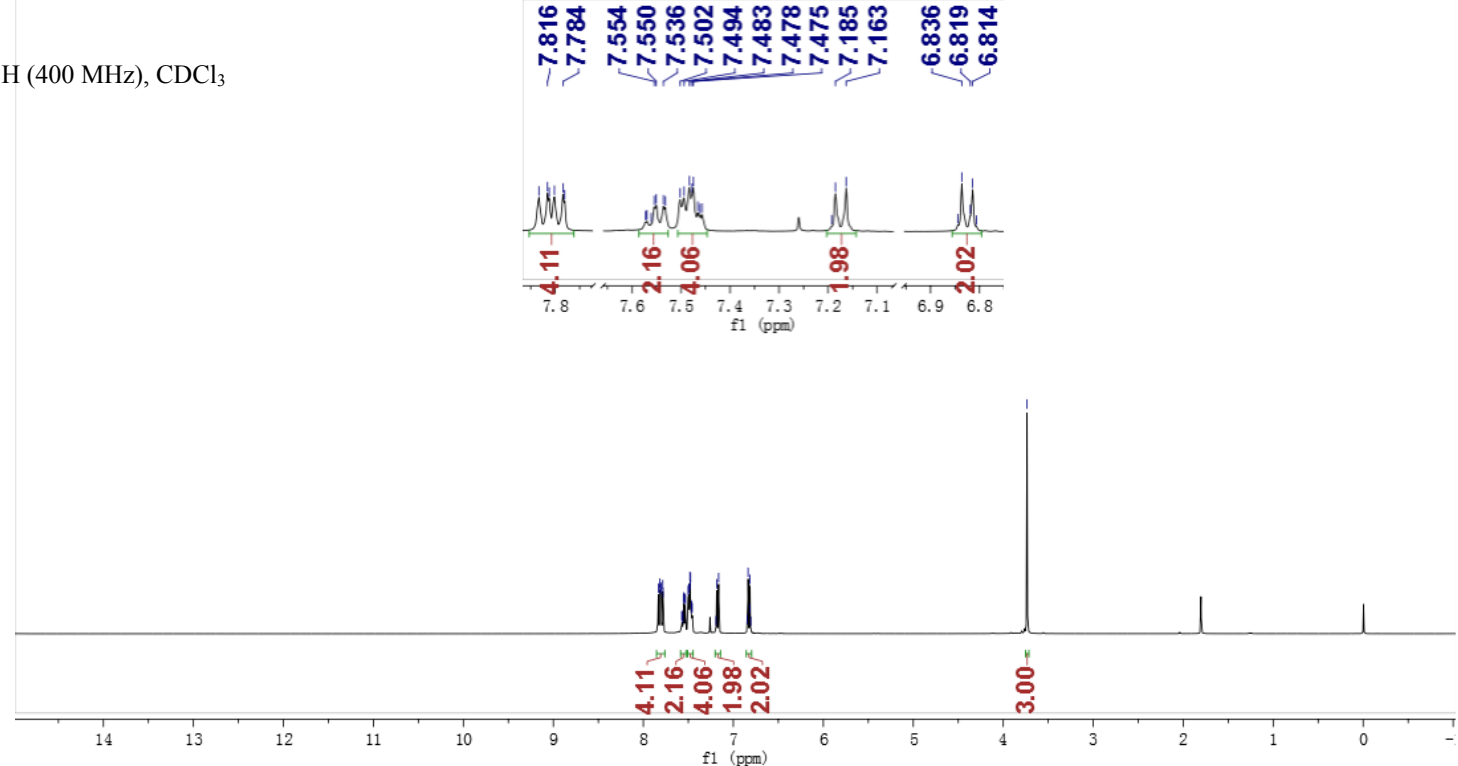
${ }^{31} \mathrm{P}(162 \mathrm{MHz}), \mathrm{CDCl}_{3}$

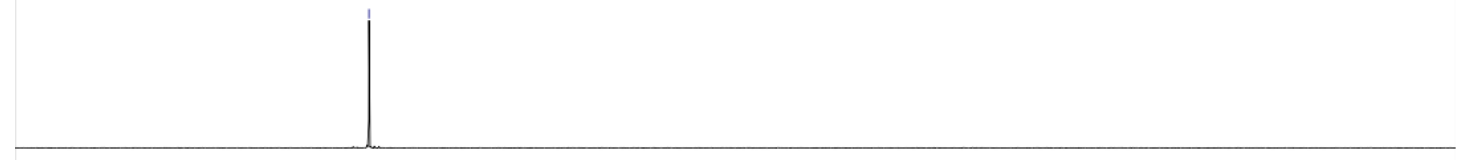

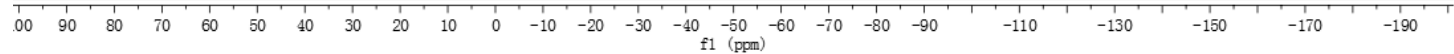

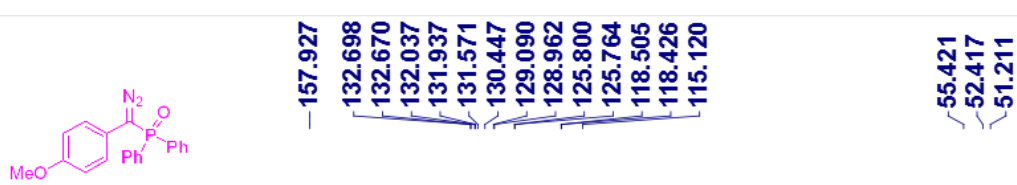

${ }^{13} \mathrm{C}(101 \mathrm{MHz}), \mathrm{CDCl}_{3}$
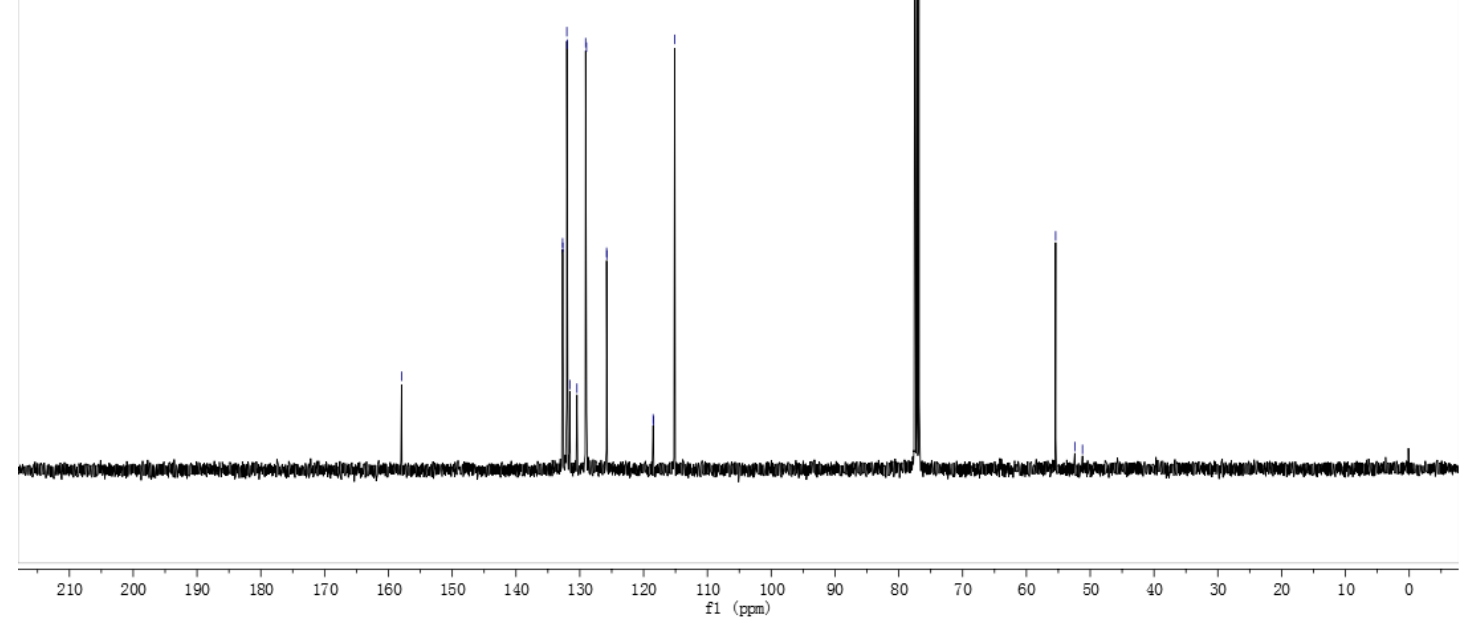

${ }^{1} \mathrm{H}(400 \mathrm{MHz}),{ }^{31} \mathrm{P}(162 \mathrm{MHz})$ and ${ }^{13} \mathrm{C}(101 \mathrm{MHz})$ spectra of compound $\mathbf{1 f}$ 


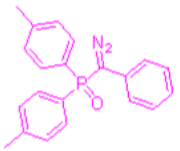

${ }^{1} \mathrm{H}(400 \mathrm{MHz}), \mathrm{CDCl}_{3}$

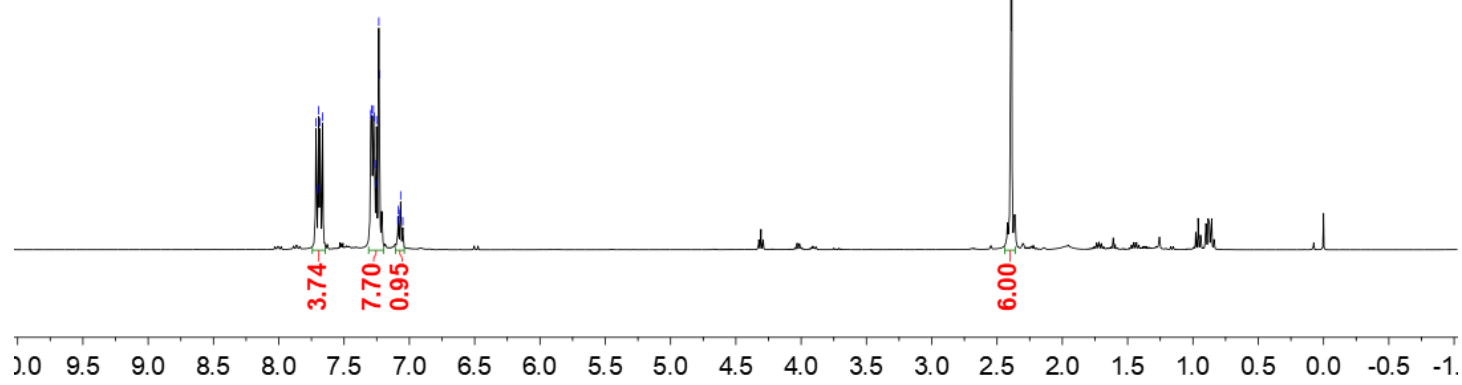

ํำ

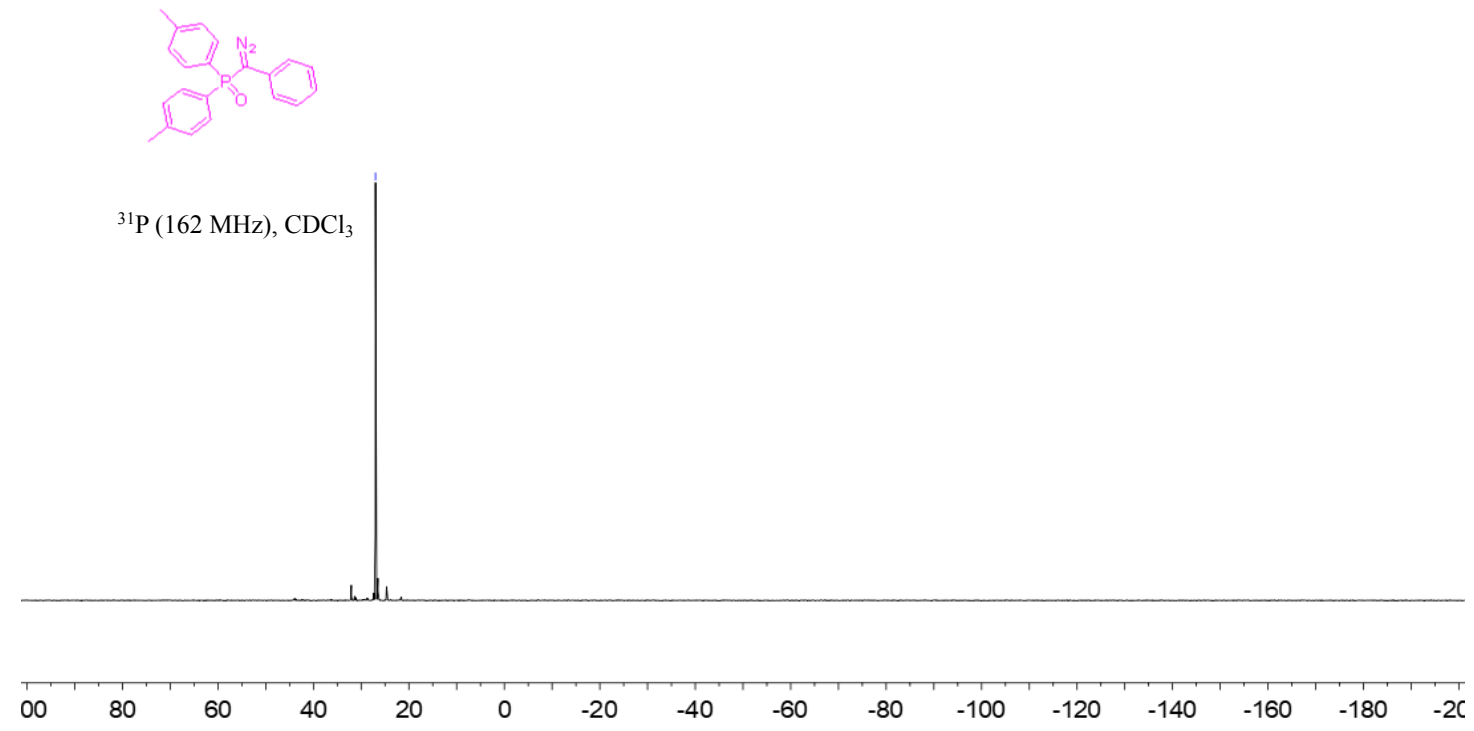




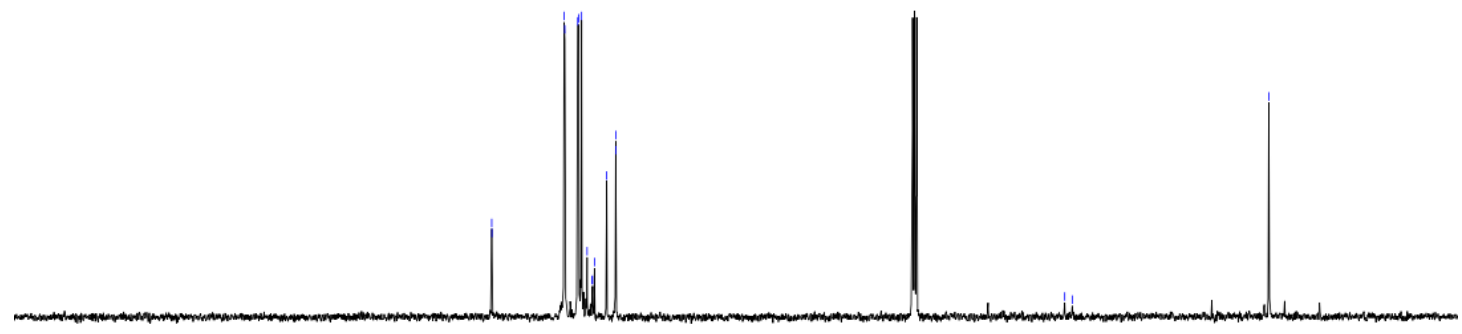

$\begin{array}{llllllllllllllllllllll}210 & 200 & 190 & 180 & 170 & 160 & 150 & 140 & 130 & 120 & 110 & 100 & 90 & 80 & 70 & 60 & 50 & 40 & 30 & 20 & 10 & 0\end{array}$

${ }^{1} \mathrm{H}(400 \mathrm{MHz}),{ }^{31} \mathrm{P}(162 \mathrm{MHz})$ and ${ }^{13} \mathrm{C}(101 \mathrm{MHz})$ spectra of compound $\mathbf{1 g}$

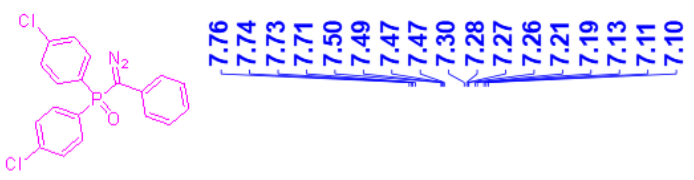

${ }^{1} \mathrm{H}(400 \mathrm{MHz}), \mathrm{CDCl}_{3}$

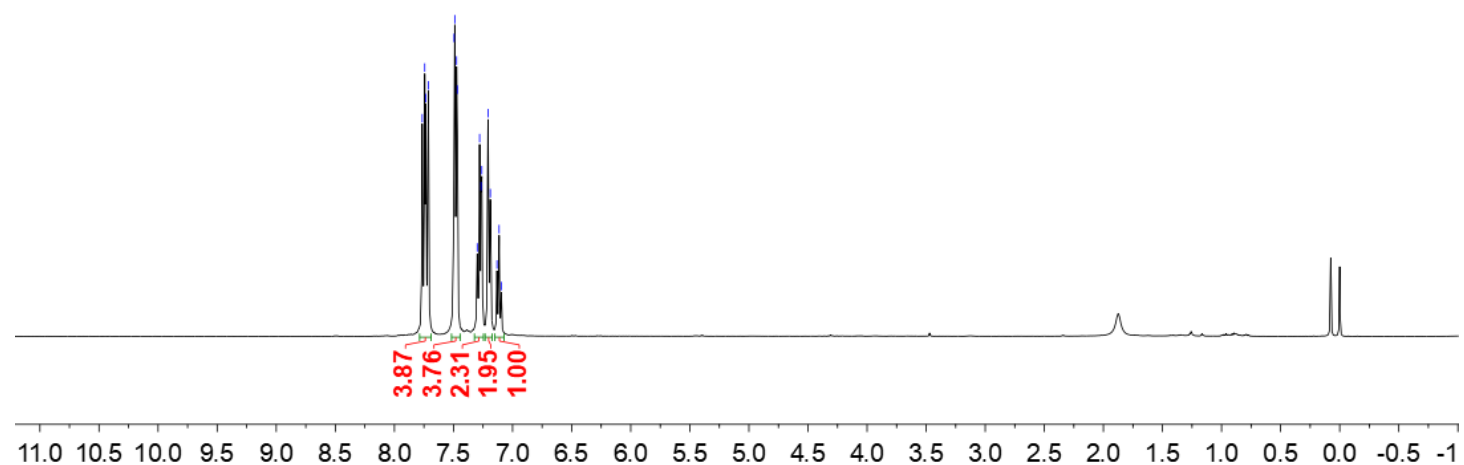


${ }^{31} \mathrm{P}(162 \mathrm{MHz}), \mathrm{CDCl}_{3}$

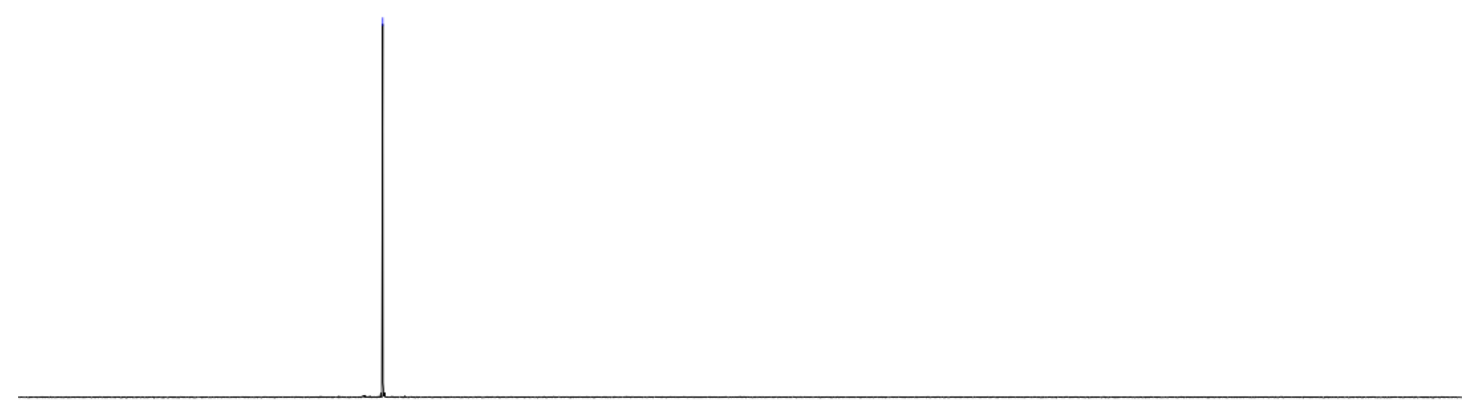

$\begin{array}{lllllllllllllllllllllll} & 00 & 80 & 60 & 40 & 20 & 0 & -20 & -40 & -60 & -80 & -100 & -120 & -140 & -160 & -180 & -26\end{array}$

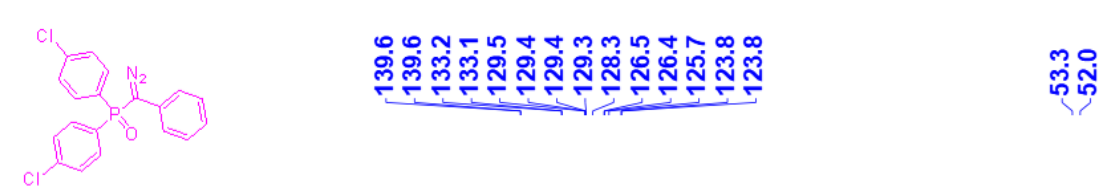

${ }^{13} \mathrm{C}(101 \mathrm{MHz}), \mathrm{CDCl}_{3}$

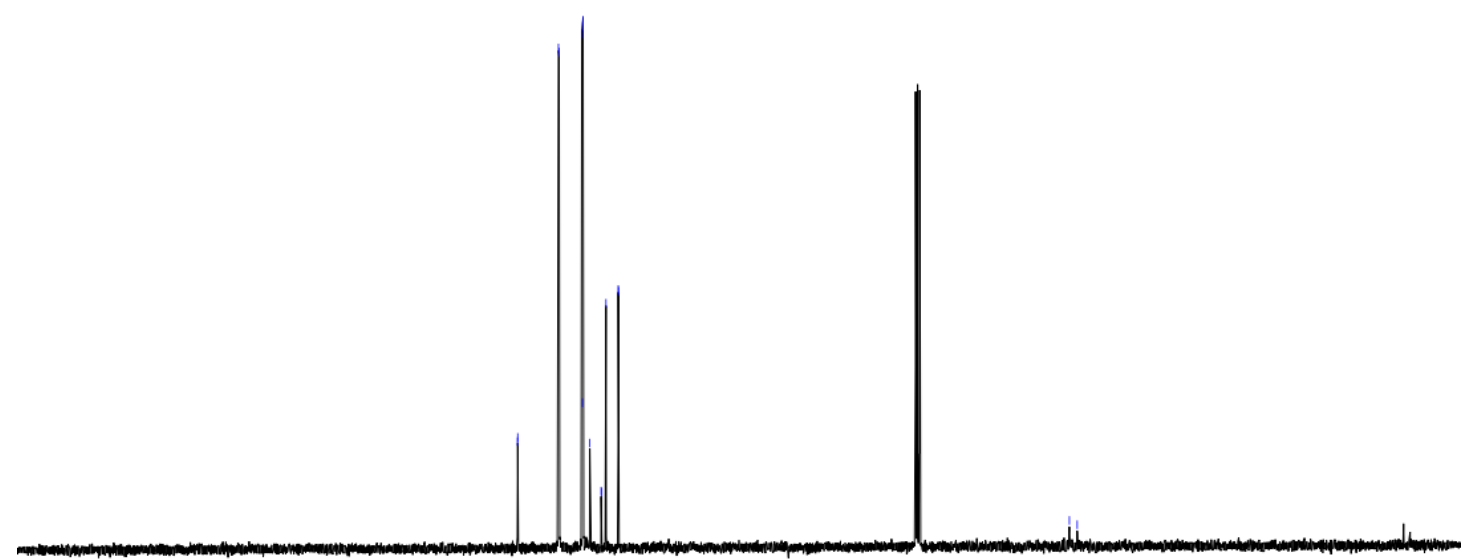

$\begin{array}{llllllllllllllllllllll}210 & 200 & 190 & 180 & 170 & 160 & 150 & 140 & 130 & 120 & 110 & 100 & 90 & 80 & 70 & 60 & 50 & 40 & 30 & 20 & 10 & 0\end{array}$

${ }^{1} \mathrm{H}(400 \mathrm{MHz}),{ }^{31} \mathrm{P}(162 \mathrm{MHz})$ and ${ }^{13} \mathrm{C}(101 \mathrm{MHz})$ spectra of compound $\mathbf{1 h}$ 


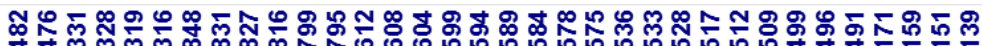

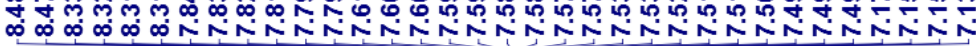

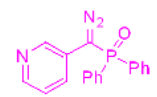

${ }^{1} \mathrm{H}(400 \mathrm{MHz}), \mathrm{CDCl}_{3}$

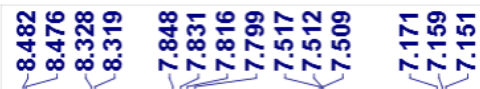

\section{$\mathrm{H}(400 \mathrm{MHz}), \mathrm{CDCl}_{3}$}
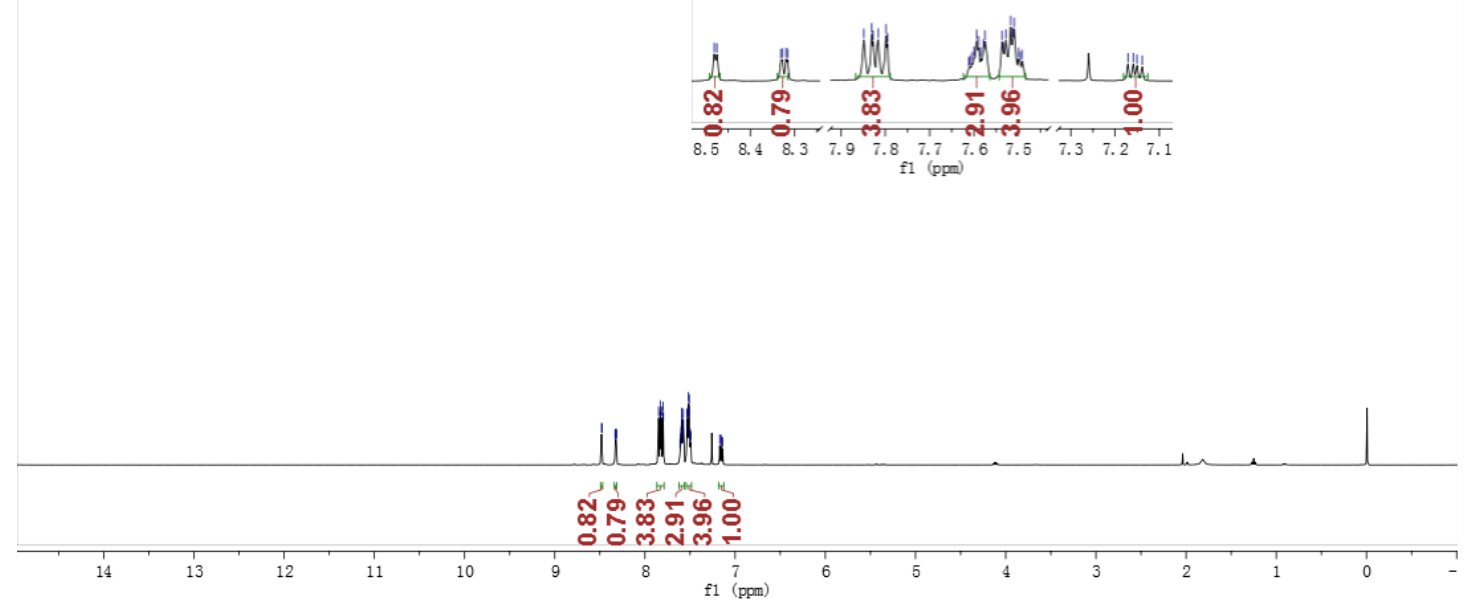

聴

${ }^{31} \mathrm{P}(162 \mathrm{MHz}), \mathrm{CDCl}_{3}$

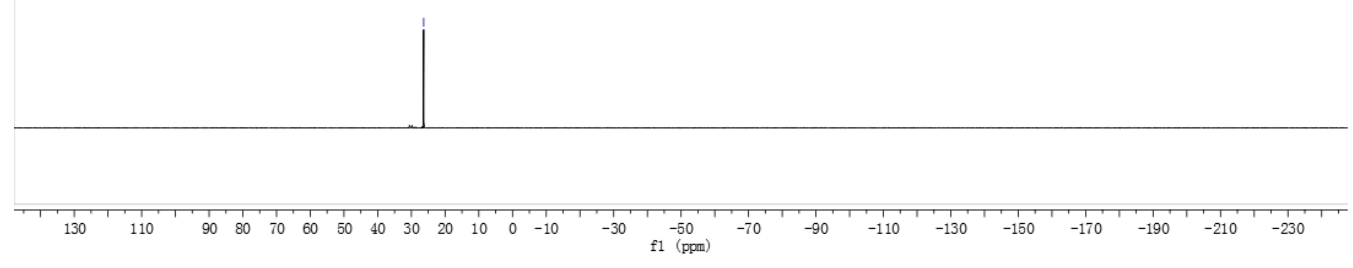




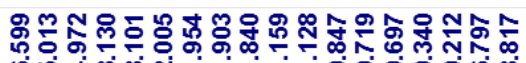

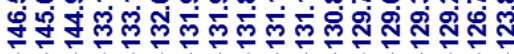

$\mathrm{N}^{\prime \prime}$

${ }^{13} \mathrm{C}$ (101 MHz), $\mathrm{CDCl}_{3}$

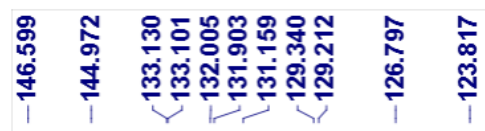
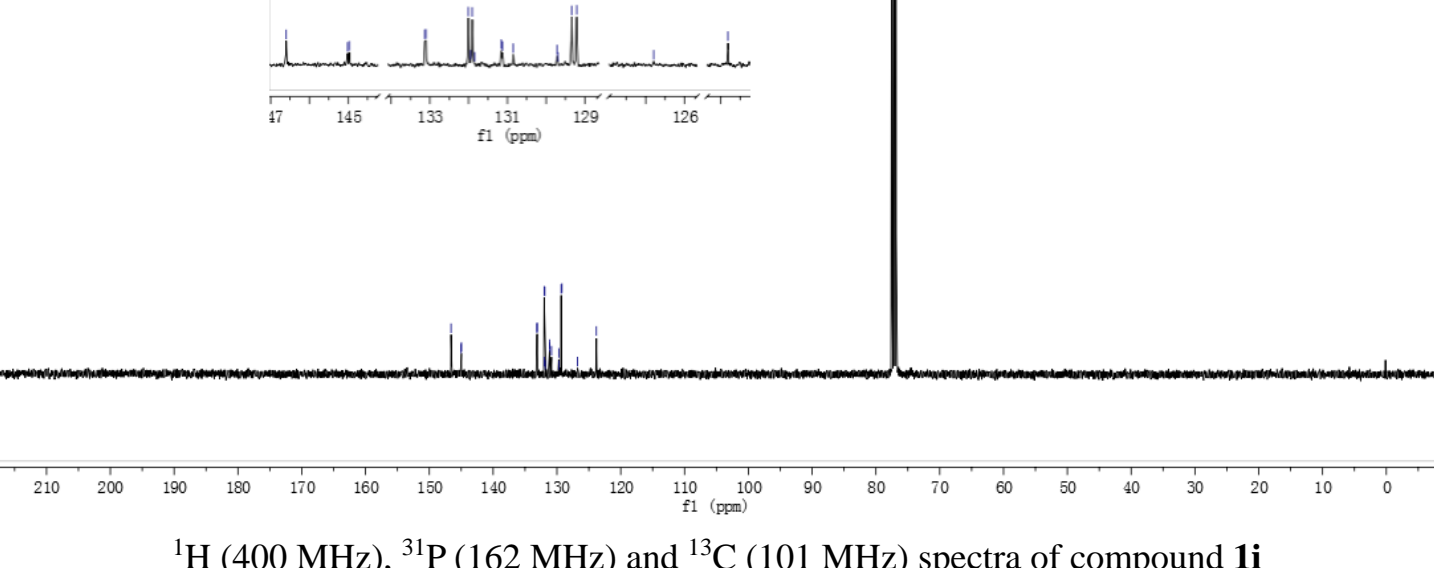

${ }^{1} \mathrm{H}(400 \mathrm{MHz}),{ }^{31} \mathrm{P}(162 \mathrm{MHz})$ and ${ }^{13} \mathrm{C}(101 \mathrm{MHz})$ spectra of compound $\mathbf{1 i}$

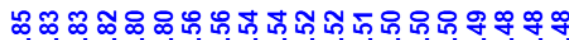

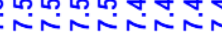

$\stackrel{\text { 윰 }}{\dot{i}}$

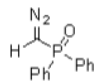

${ }^{1} \mathrm{H}(400 \mathrm{MHz}), \mathrm{CDCl}_{3}$

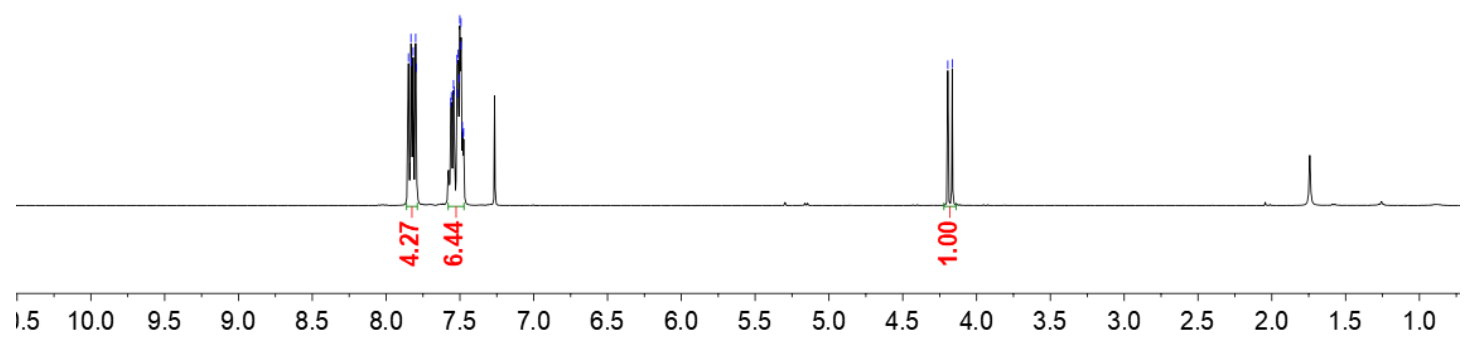




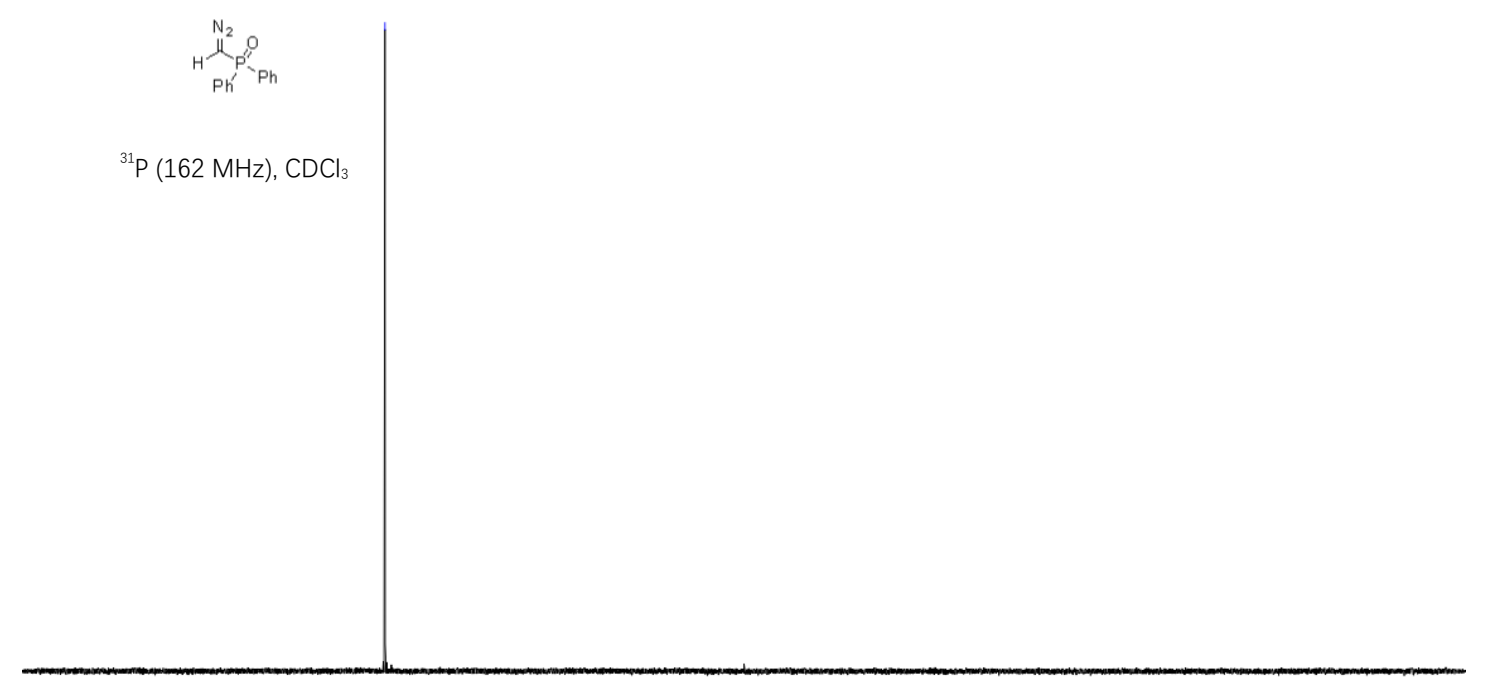

\begin{tabular}{lllllllllllllllll}
\hline 0 & 80 & 60 & 40 & 20 & 0 & -20 & -40 & -60 & -80 & -100 & -120 & -140 & -160 & -180 & -20
\end{tabular}

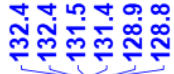

$$
\mathrm{Hh}_{\mathrm{Ph}}^{\mathrm{N}_{2} \mathrm{O}} \mathrm{O}
$$

${ }^{13} \mathrm{C}(101 \mathrm{MHz}), \mathrm{CDCl}_{3}$

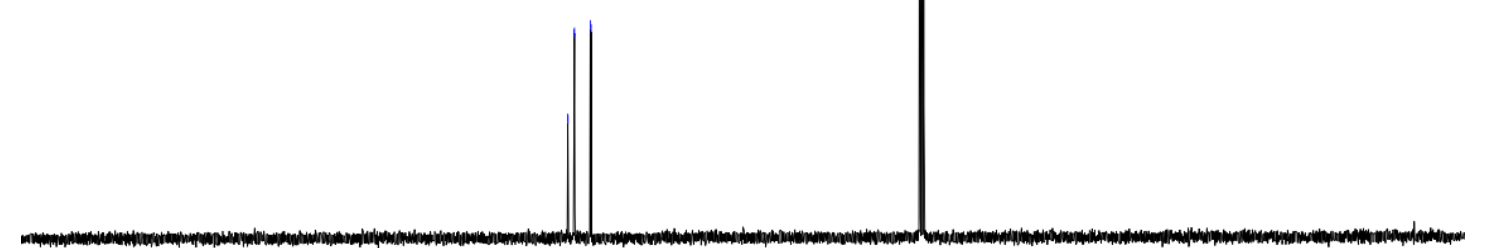

$\begin{array}{llllllllllllllllllllll}210 & 200 & 190 & 180 & 170 & 160 & 150 & 140 & 130 & 120 & 110 & 100 & 90 & 80 & 70 & 60 & 50 & 40 & 30 & 20 & 10 & 0\end{array}$

${ }^{1} \mathrm{H}(400 \mathrm{MHz}),{ }^{31} \mathrm{P}(162 \mathrm{MHz})$ and ${ }^{13} \mathrm{C}(101 \mathrm{MHz})$ spectra of compound $\mathbf{1 j}$ 


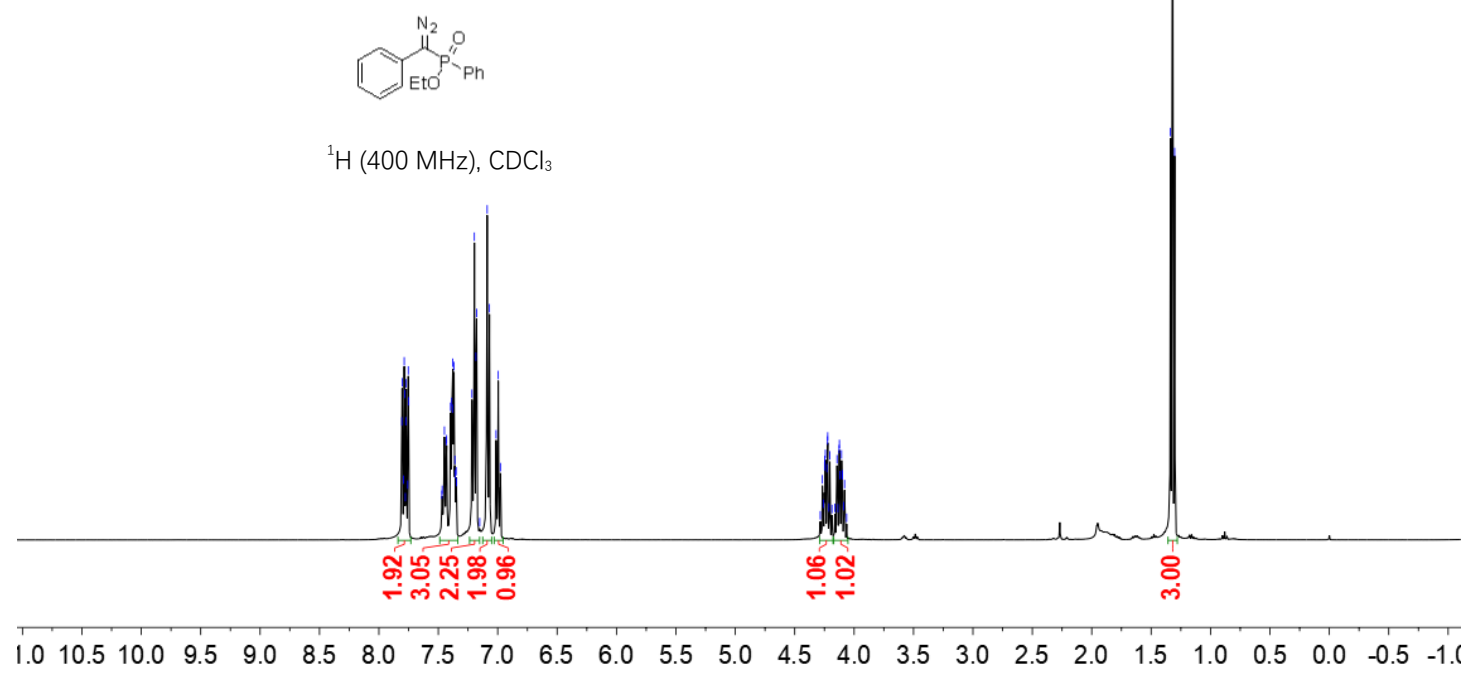

\section{萬}

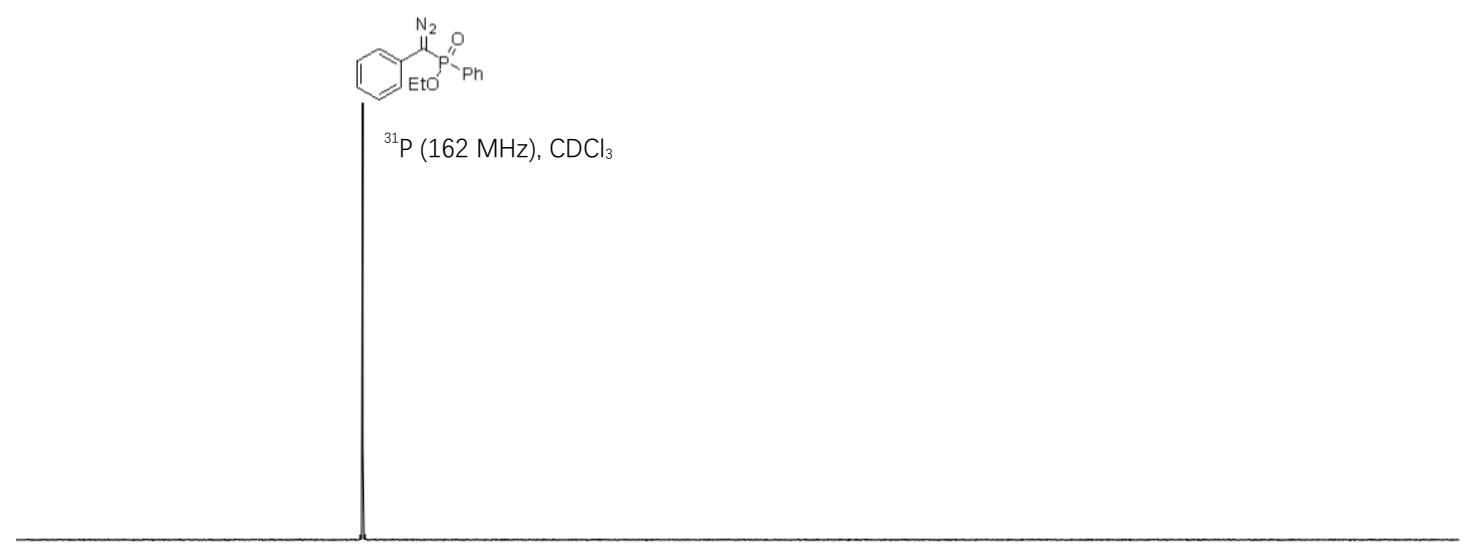

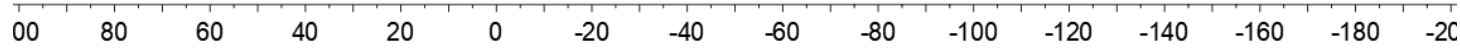




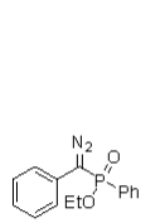

${ }^{13} \mathrm{C}(101 \mathrm{MHz}), \mathrm{CDCl}_{3}$

${ }^{1} \mathrm{H}(400 \mathrm{MHz}),{ }^{31} \mathrm{P}(162 \mathrm{MHz})$ and ${ }^{13} \mathrm{C}(101 \mathrm{MHz})$ spectra of compound $\mathbf{1 k}$

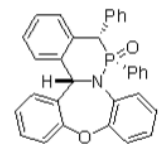

${ }^{1} \mathrm{H}(400 \mathrm{MHz}), \mathrm{CDCl}_{3}$

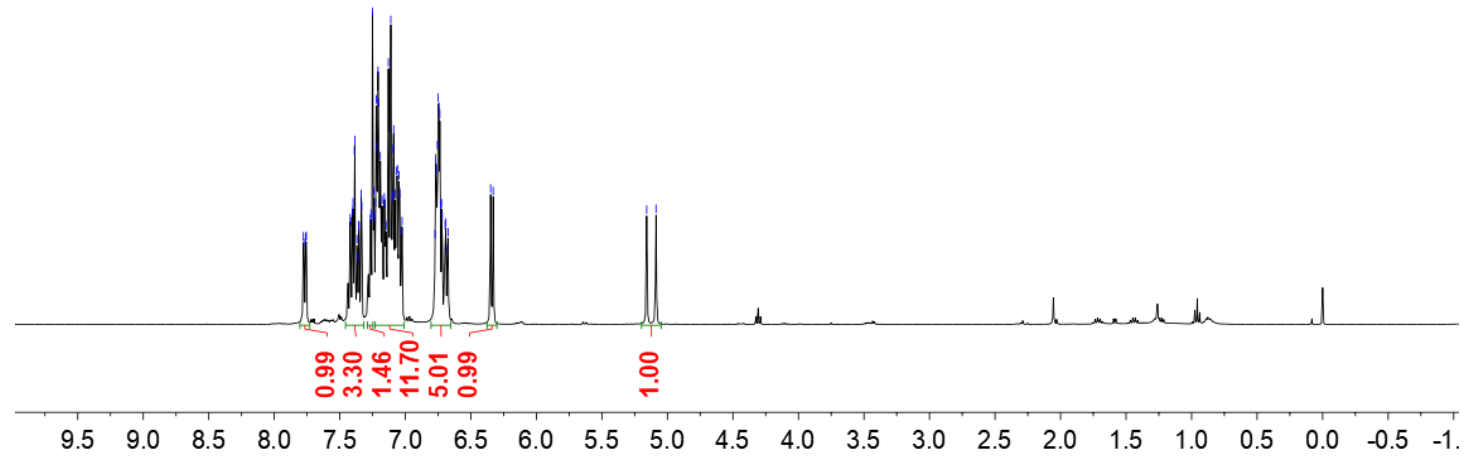




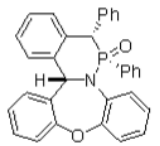

${ }^{31} \mathrm{P}(162 \mathrm{MHz}), \mathrm{CDCl}_{3}$

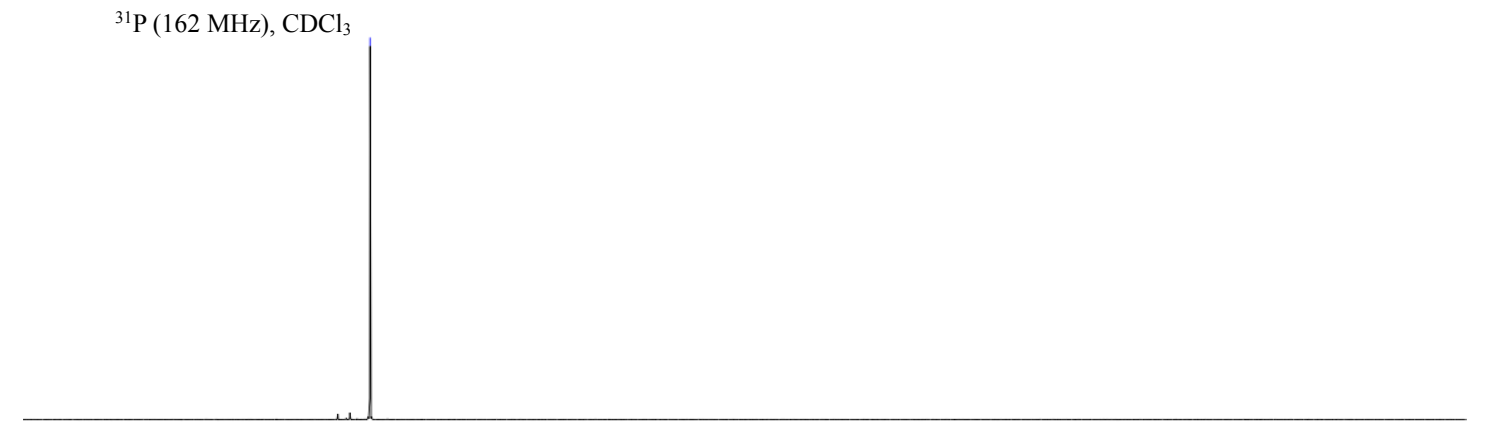

\begin{tabular}{llllllllllllllll}
\hline 00 & 80 & 60 & 40 & 20 & 0 & -20 & -40 & -60 & -80 & -100 & -120 & -140 & -160 & -180 & -20
\end{tabular}

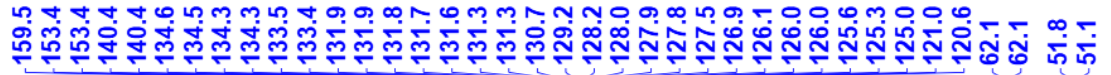

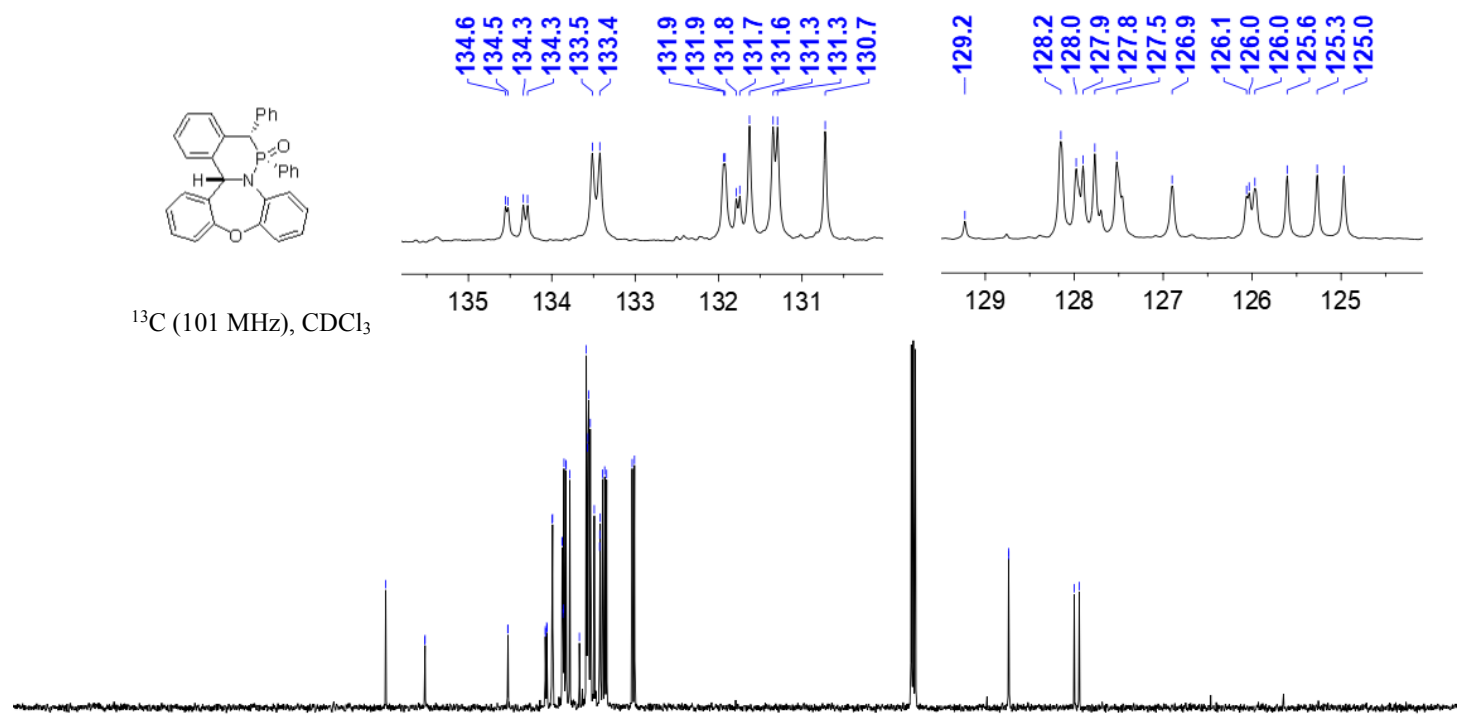

$\begin{array}{llllllllllllllllllllll}210 & 200 & 190 & 180 & 170 & 160 & 150 & 140 & 130 & 120 & 110 & 100 & 90 & 80 & 70 & 60 & 50 & 40 & 30 & 20 & 10 & 0\end{array}$

${ }^{1} \mathrm{H}(400 \mathrm{MHz}),{ }^{31} \mathrm{P}(162 \mathrm{MHz})$ and ${ }^{13} \mathrm{C}(101 \mathrm{MHz})$ spectra of compound 3a 


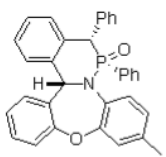

${ }^{1} \mathrm{H}(400 \mathrm{MHz}), \mathrm{CDCl}_{3}$

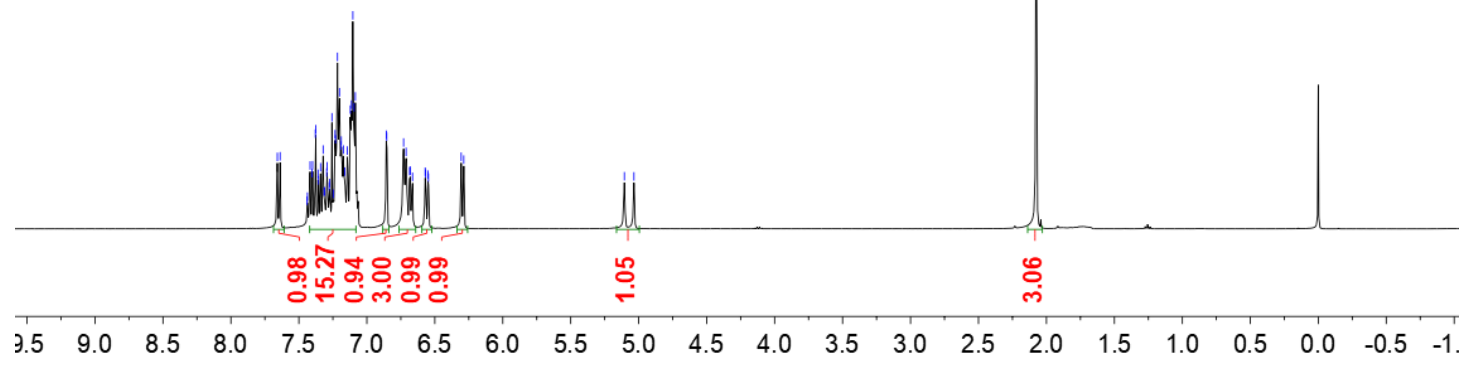

$\stackrel{\text { ஸ }}{\stackrel{1}{\sim}}$

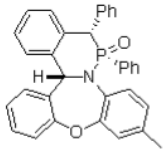

${ }^{31} \mathrm{P}(162 \mathrm{MHz}), \mathrm{CDCl}_{3}$

\begin{tabular}{llllllllllllllllllllll}
\hline 0 & 80 & 60 & 40 & 20 & 0 & -20 & -40 & -60 & -80 & -100 & -120 & -140 & -160 & -180 & -20
\end{tabular}




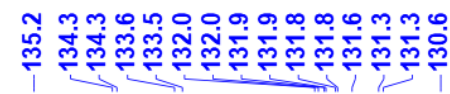
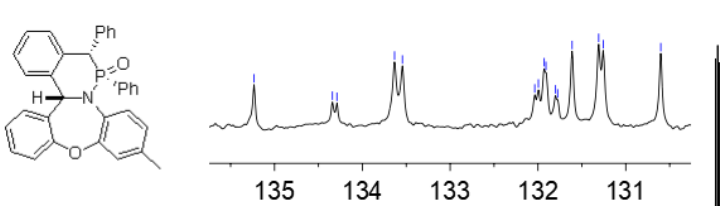

m.

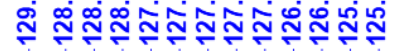

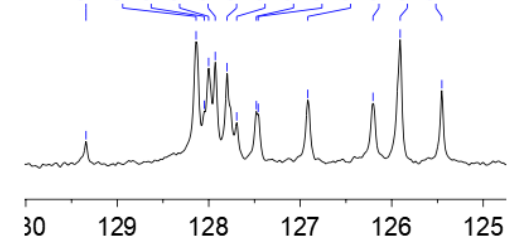

${ }^{13} \mathrm{C}(101 \mathrm{MHz}), \mathrm{CDCl}_{3}$

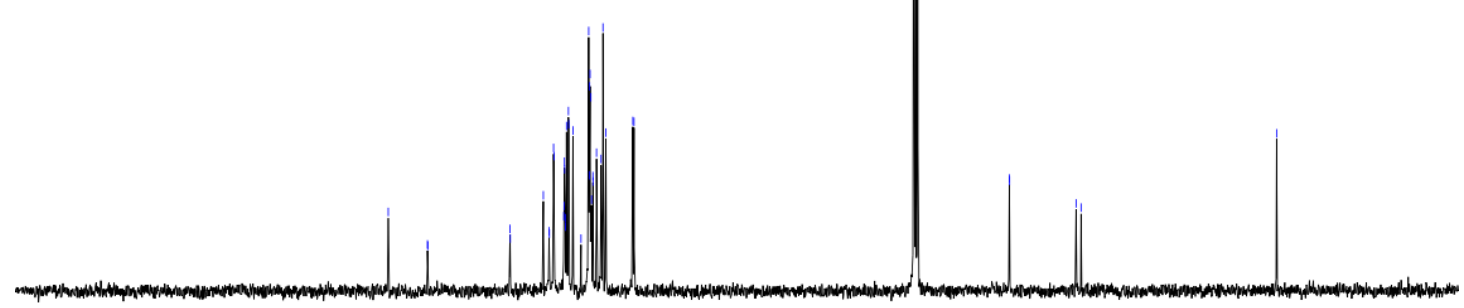

$\begin{array}{llllllllllllllllllllll}210 & 200 & 190 & 180 & 170 & 160 & 150 & 140 & 130 & 120 & 110 & 100 & 90 & 80 & 70 & 60 & 50 & 40 & 30 & 20 & 10 & 0\end{array}$

${ }^{1} \mathrm{H}(400 \mathrm{MHz}),{ }^{31} \mathrm{P}(162 \mathrm{MHz})$ and ${ }^{13} \mathrm{C}(101 \mathrm{MHz})$ spectra of compound $\mathbf{3 b}$

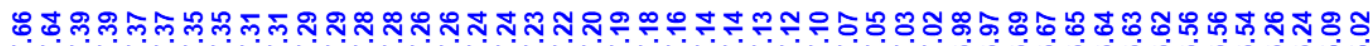

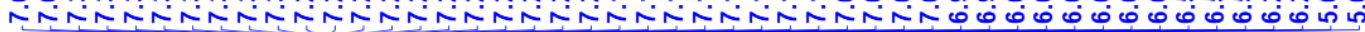

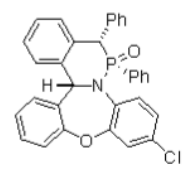

${ }^{1} \mathrm{H}(400 \mathrm{MHz}), \mathrm{CDCl}_{3}$

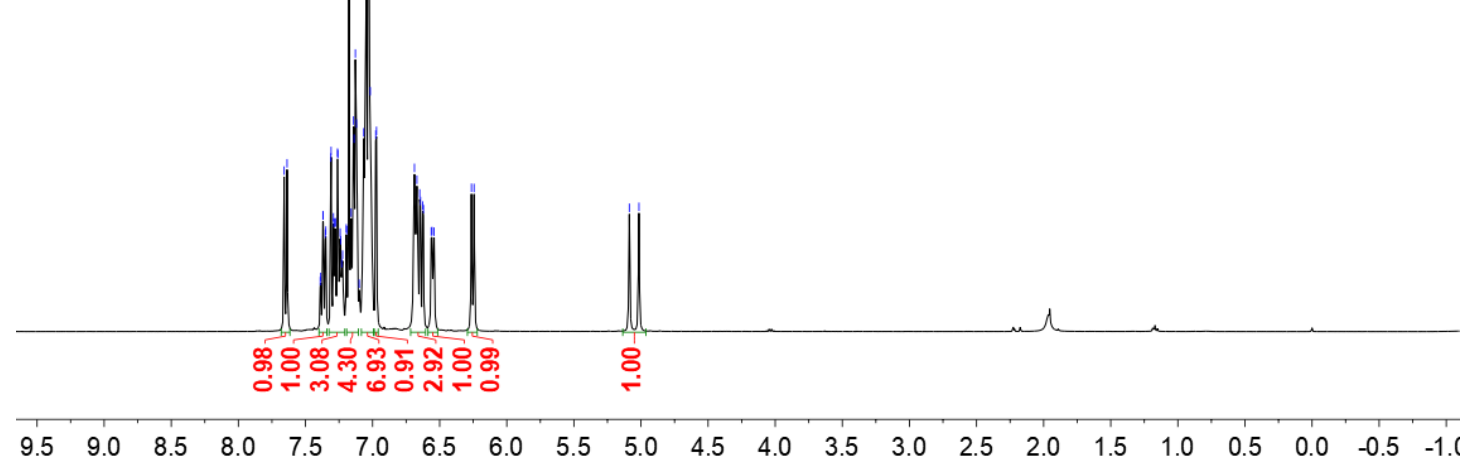




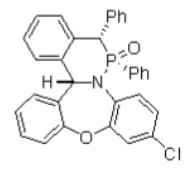

${ }^{31} \mathrm{P}(162 \mathrm{MHz}), \mathrm{CDCl}_{3}$

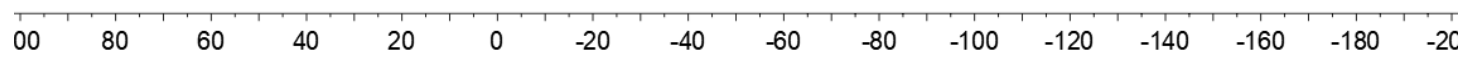

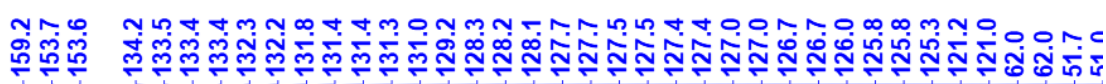
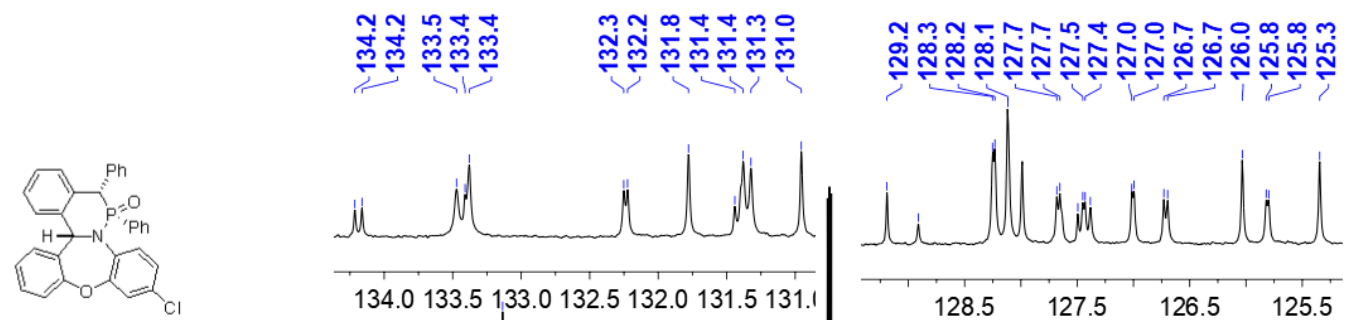

${ }^{13} \mathrm{C}(101 \mathrm{MHz}), \mathrm{CDCl}_{3}$
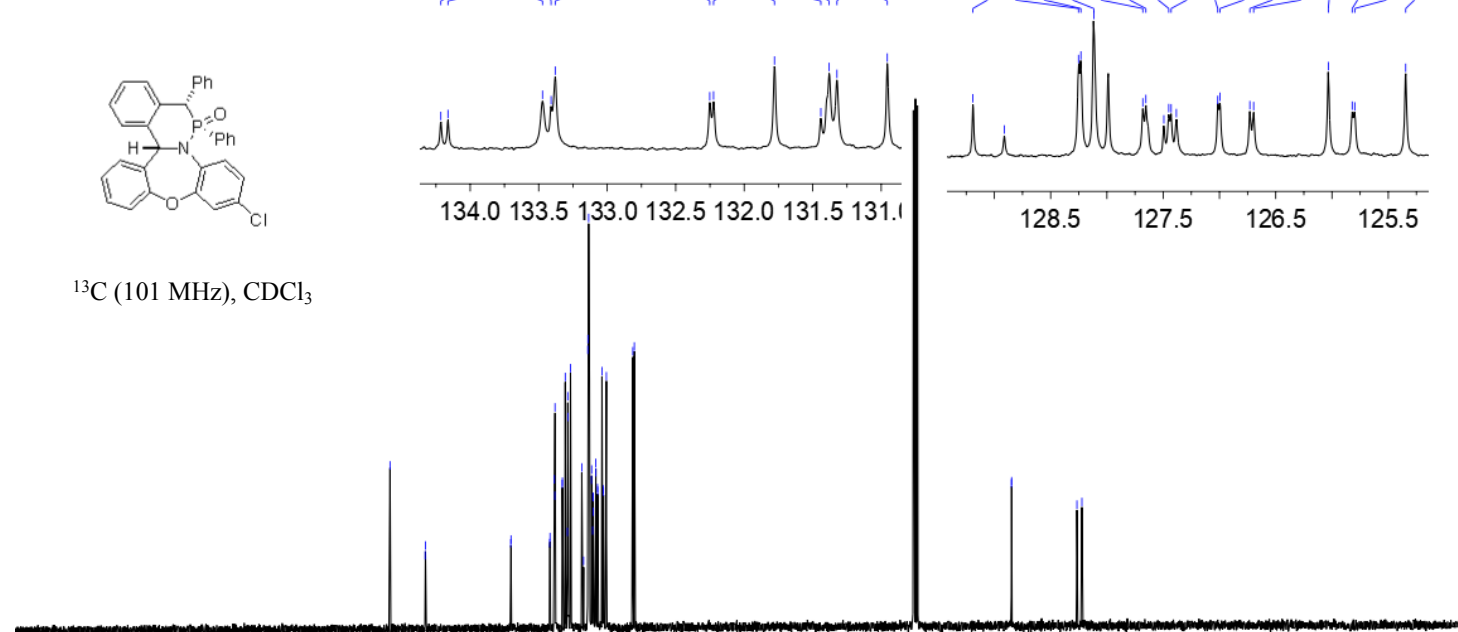

$\begin{array}{llllllllllllllllllllll}210 & 200 & 190 & 180 & 170 & 160 & 150 & 140 & 130 & 120 & 110 & 100 & 90 & 80 & 70 & 60 & 50 & 40 & 30 & 20 & 10 & 0\end{array}$

${ }^{1} \mathrm{H}(400 \mathrm{MHz}),{ }^{31} \mathrm{P}(162 \mathrm{MHz})$ and ${ }^{13} \mathrm{C}(101 \mathrm{MHz})$ spectra of compound 3c 


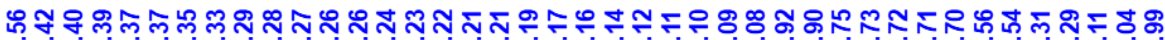

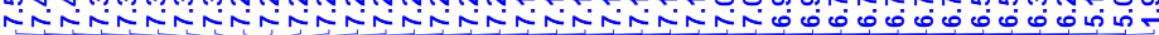

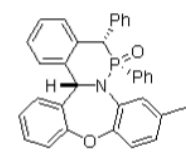

${ }^{1} \mathrm{H}(400 \mathrm{MHz}), \mathrm{CDCl}_{3}$

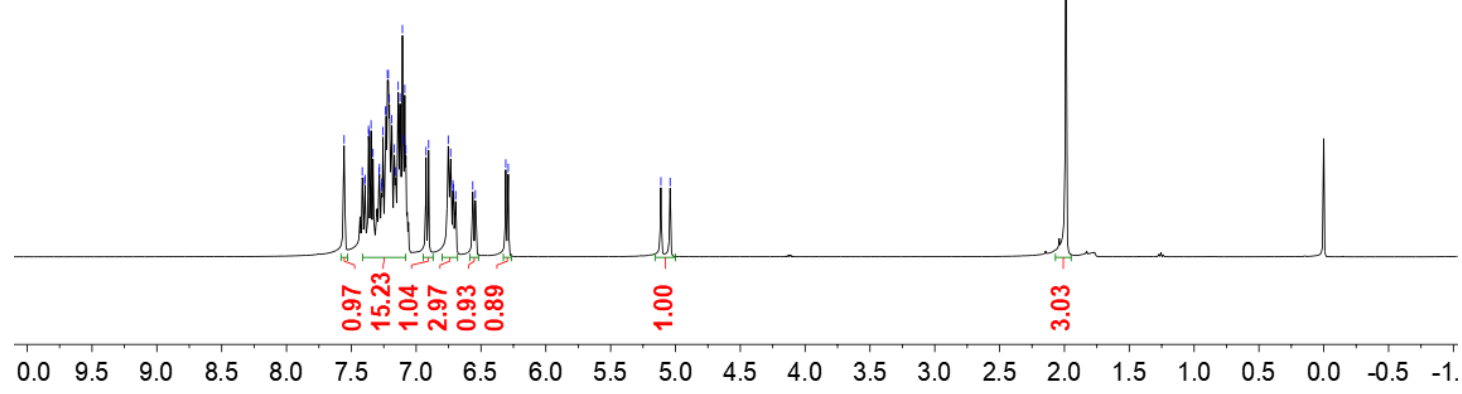

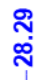

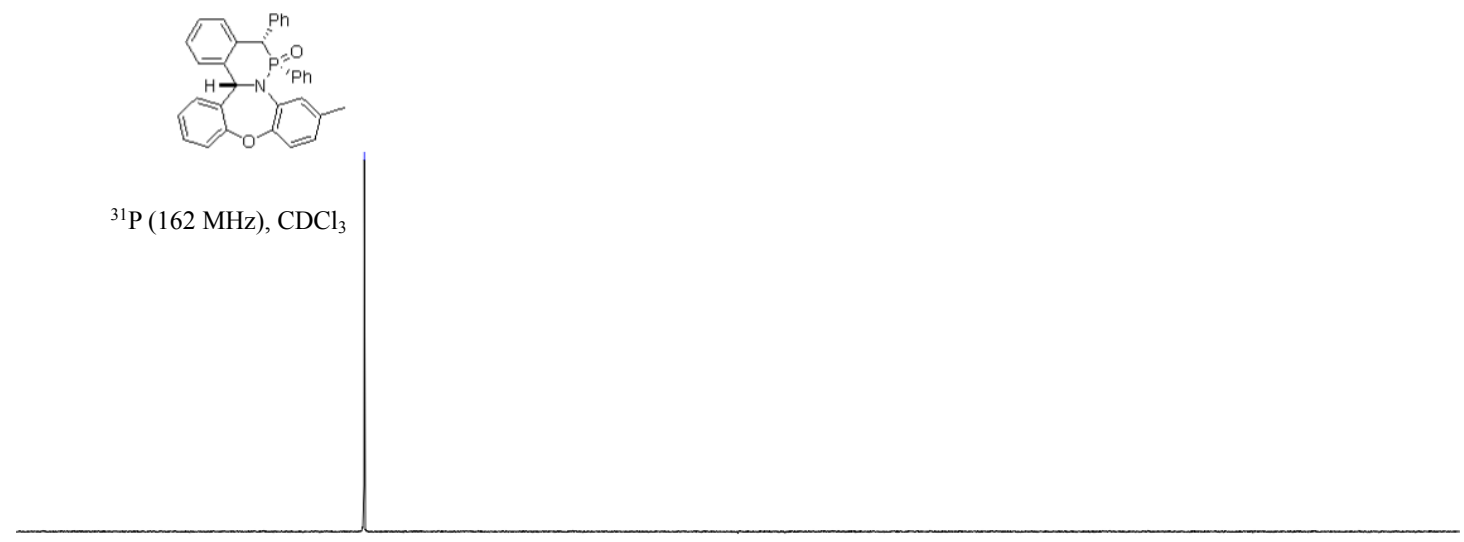

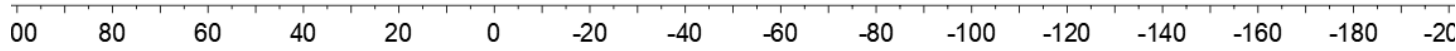



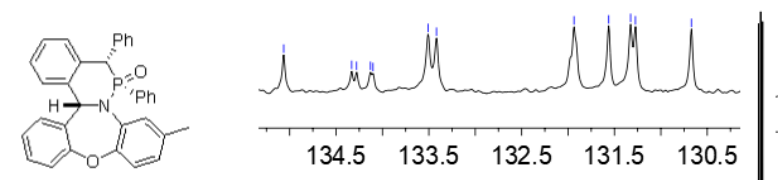

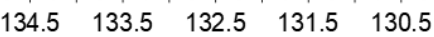

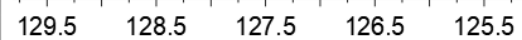

${ }^{13} \mathrm{C}(101 \mathrm{MHz}), \mathrm{CDCl}_{3}$

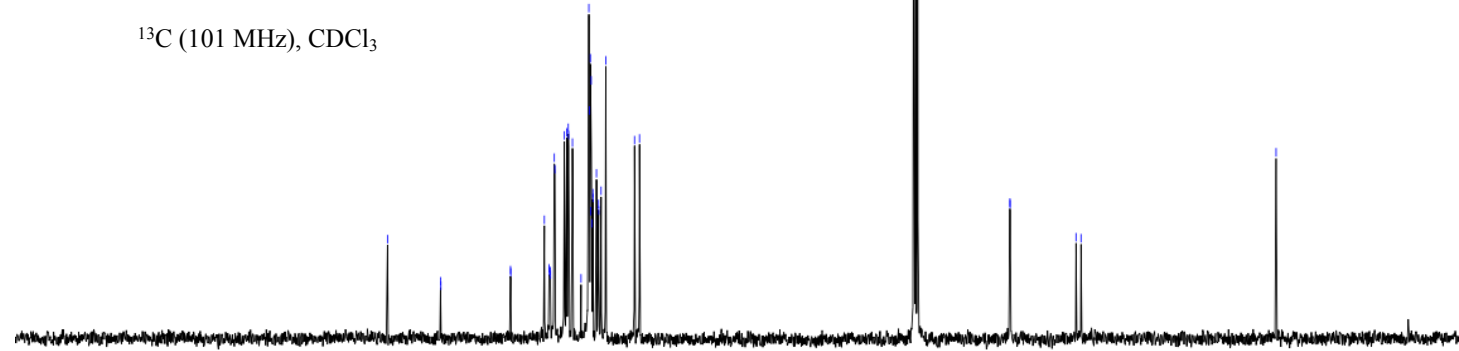

$\begin{array}{llllllllllllllllllllll}210 & 200 & 190 & 180 & 170 & 160 & 150 & 140 & 130 & 120 & 110 & 100 & 90 & 80 & 70 & 60 & 50 & 40 & 30 & 20 & 10 & 0\end{array}$

${ }^{1} \mathrm{H}(400 \mathrm{MHz}),{ }^{31} \mathrm{P}(162 \mathrm{MHz})$ and ${ }^{13} \mathrm{C}(101 \mathrm{MHz})$ spectra of compound 3d

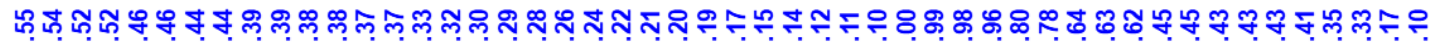

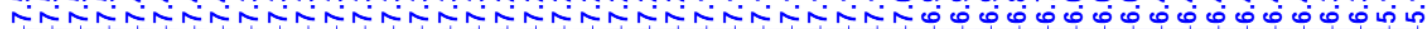

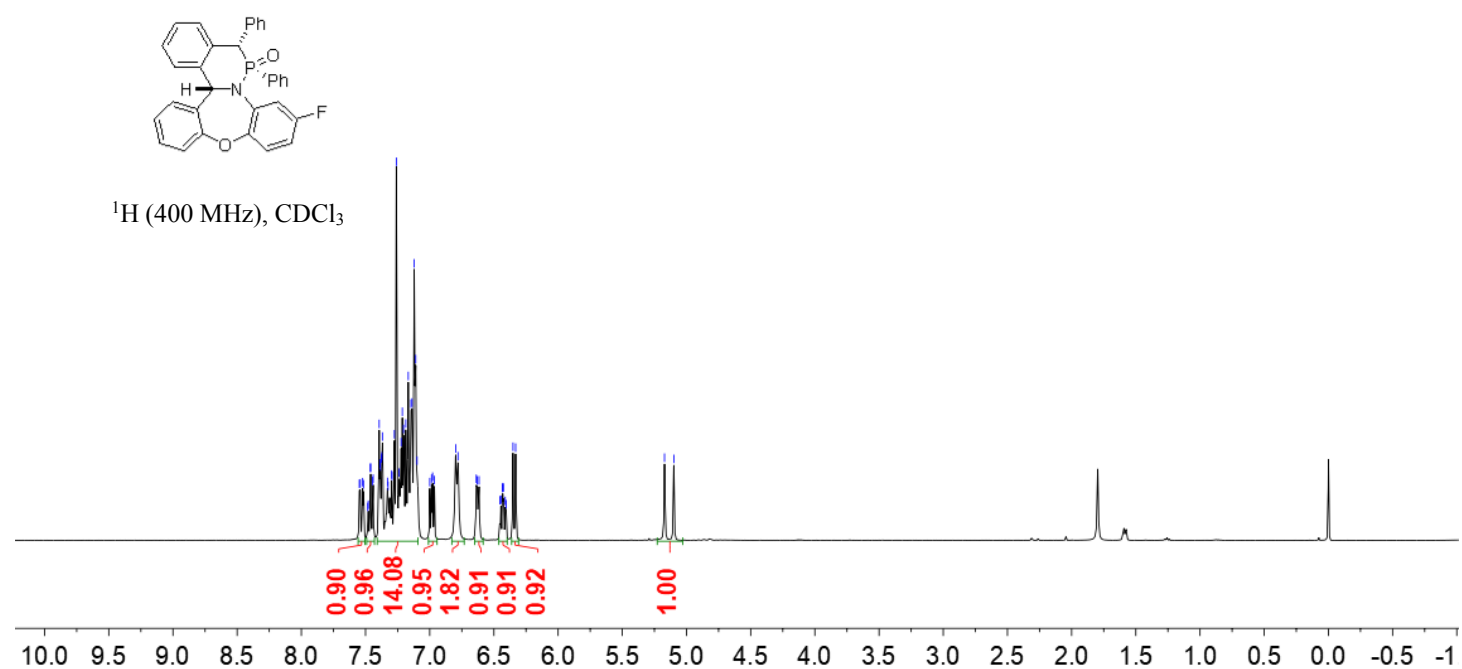




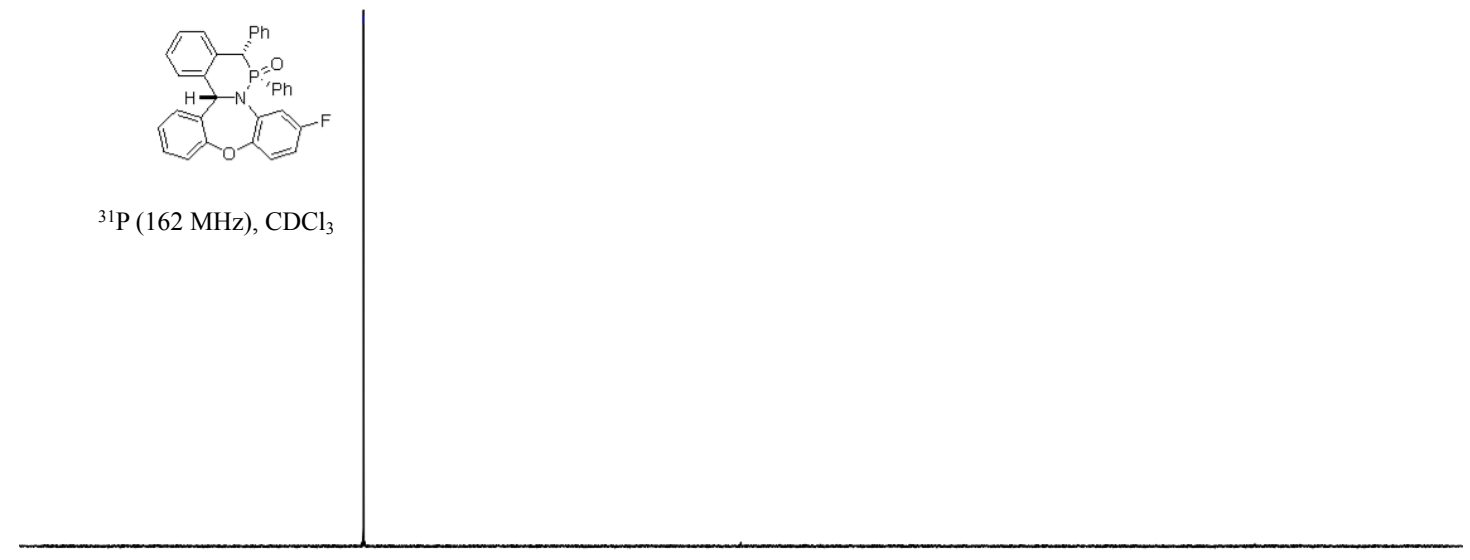

$\begin{array}{lllllllllllllllll} & 00 & 80 & 60 & 40 & 20 & 0 & -20 & -40 & -60 & -80 & -100 & -120 & -140 & -160 & -180 & -20\end{array}$

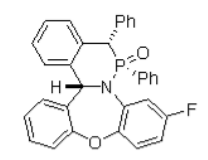

${ }^{19} \mathrm{~F}(376 \mathrm{MHz}), \mathrm{CDCl}_{3}$ 


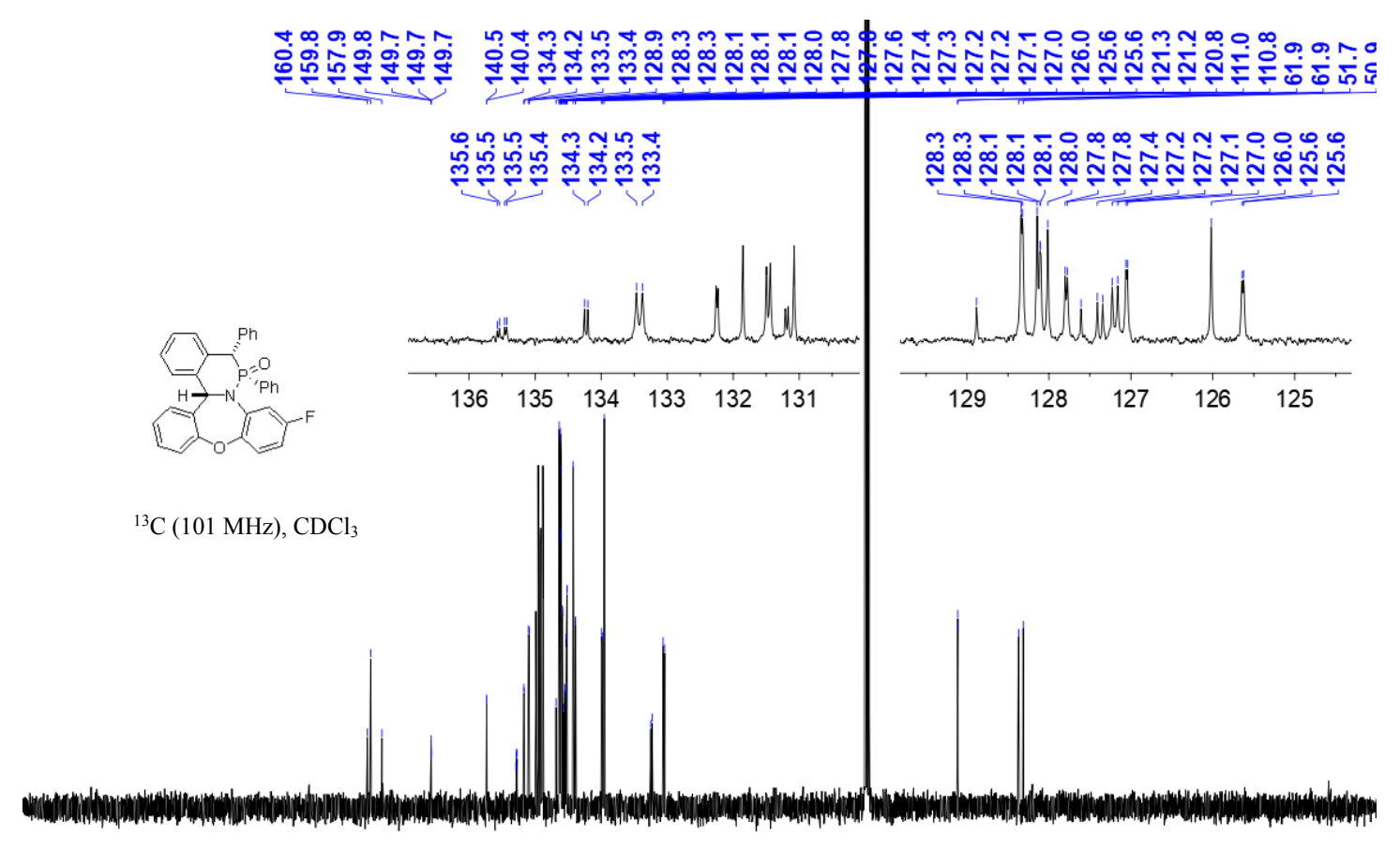

$\begin{array}{llllllllllllllllllllll}210 & 200 & 190 & 180 & 170 & 160 & 150 & 140 & 130 & 120 & 110 & 100 & 90 & 80 & 70 & 60 & 50 & 40 & 30 & 20 & 10 & 0\end{array}$

${ }^{1} \mathrm{H}(400 \mathrm{MHz}),{ }^{31} \mathrm{P}(162 \mathrm{MHz}),{ }^{19} \mathrm{~F}$ NMR $(376 \mathrm{MHz})$ and ${ }^{13} \mathrm{C}(101 \mathrm{MHz})$ spectra of compound 3e

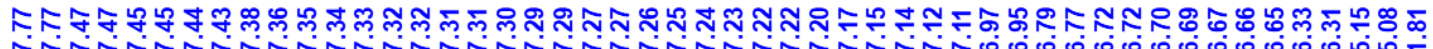

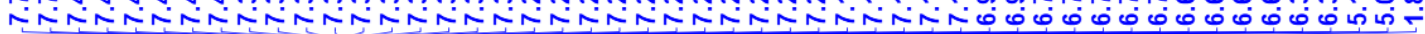

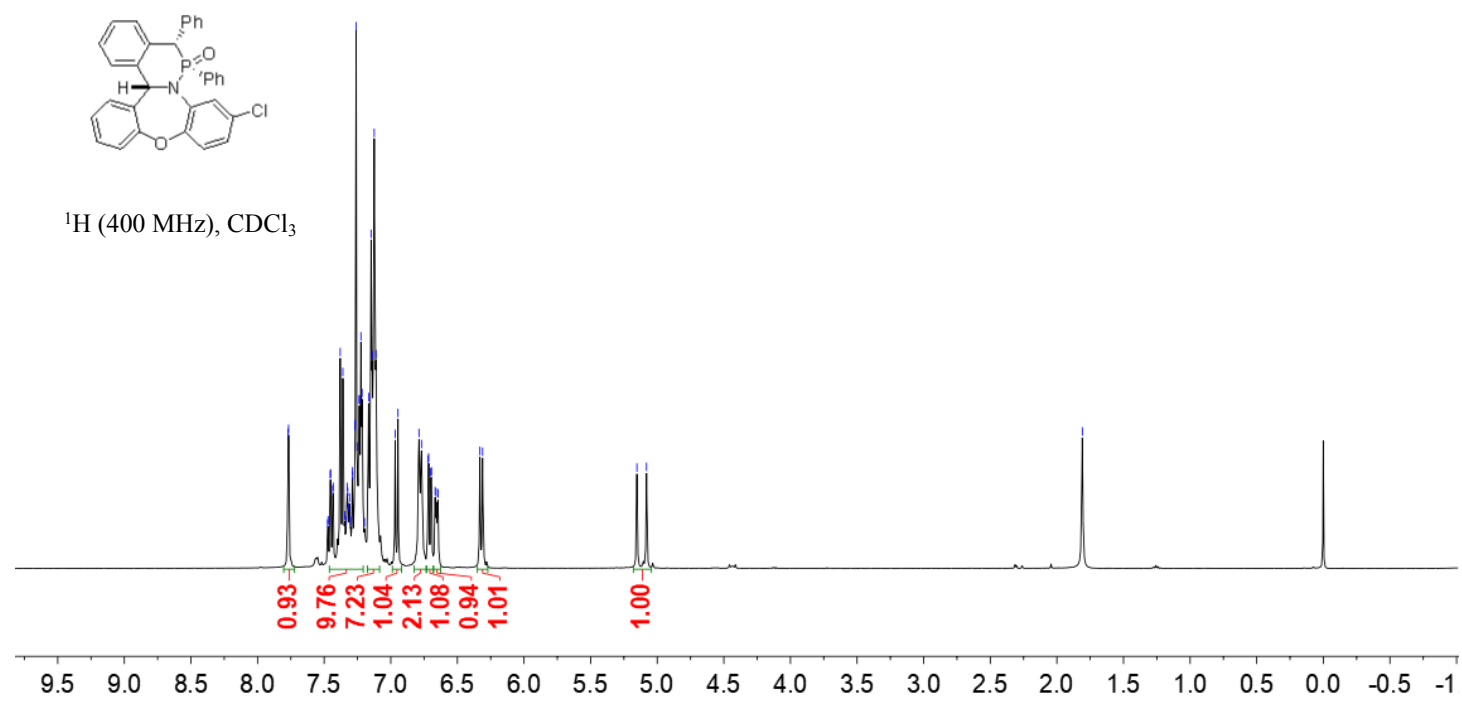




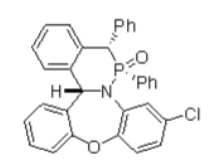

${ }^{31} \mathrm{P}(162 \mathrm{MHz}), \mathrm{CDCl}_{3}$

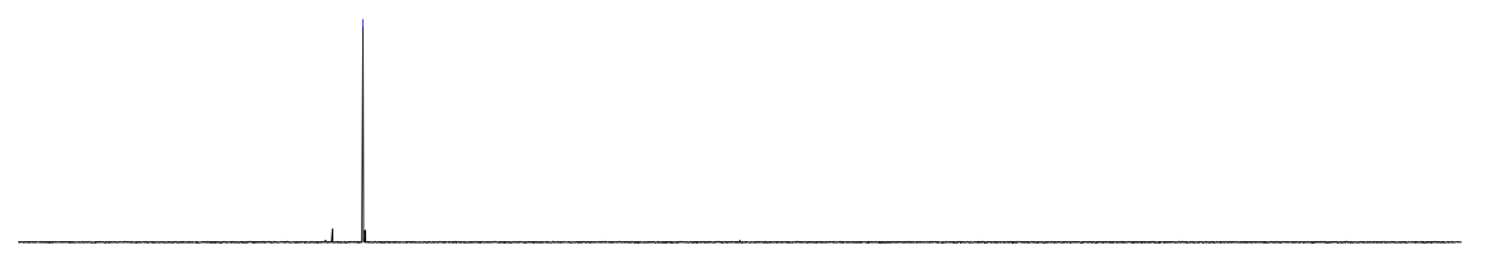

$\begin{array}{llllllllllllllllll} & 00 & 80 & 60 & 40 & 20 & 0 & -20 & -40 & -60 & -80 & -100 & -120 & -140 & -160 & -180 & -2 C\end{array}$

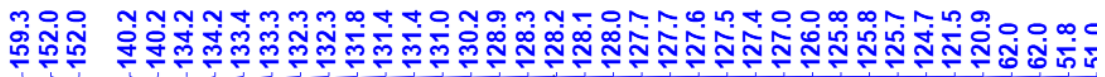

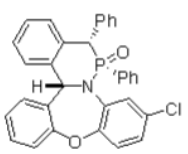

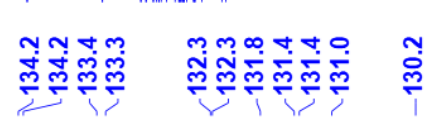

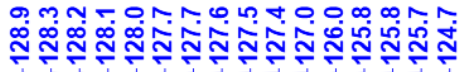

${ }^{13} \mathrm{C}$ (101 MHz), $\mathrm{CDCl}_{3}$
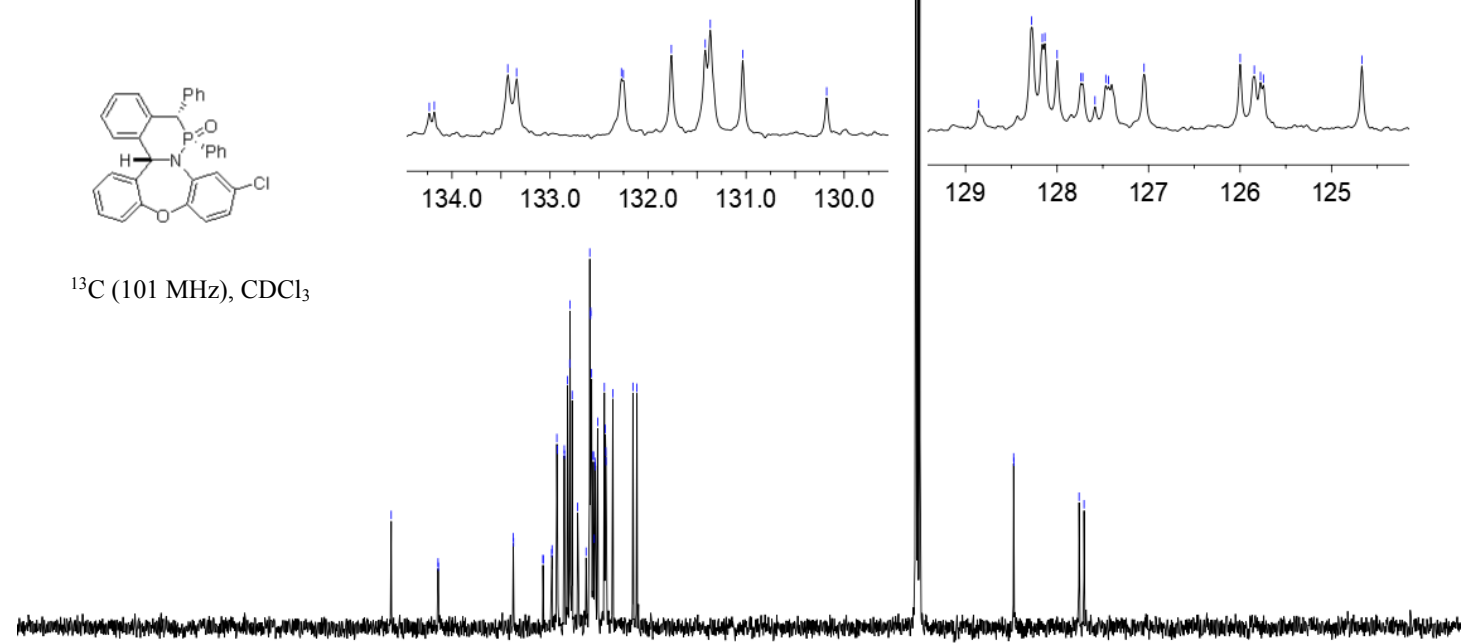

$\begin{array}{llllllllllllllllllllll}210 & 200 & 190 & 180 & 170 & 160 & 150 & 140 & 130 & 120 & 110 & 100 & 90 & 80 & 70 & 60 & 50 & 40 & 30 & 20 & 10 & 0\end{array}$

${ }^{1} \mathrm{H}(400 \mathrm{MHz}),{ }^{31} \mathrm{P}(162 \mathrm{MHz})$ and ${ }^{13} \mathrm{C}(101 \mathrm{MHz})$ spectra of compound $\mathbf{3 f}$ 


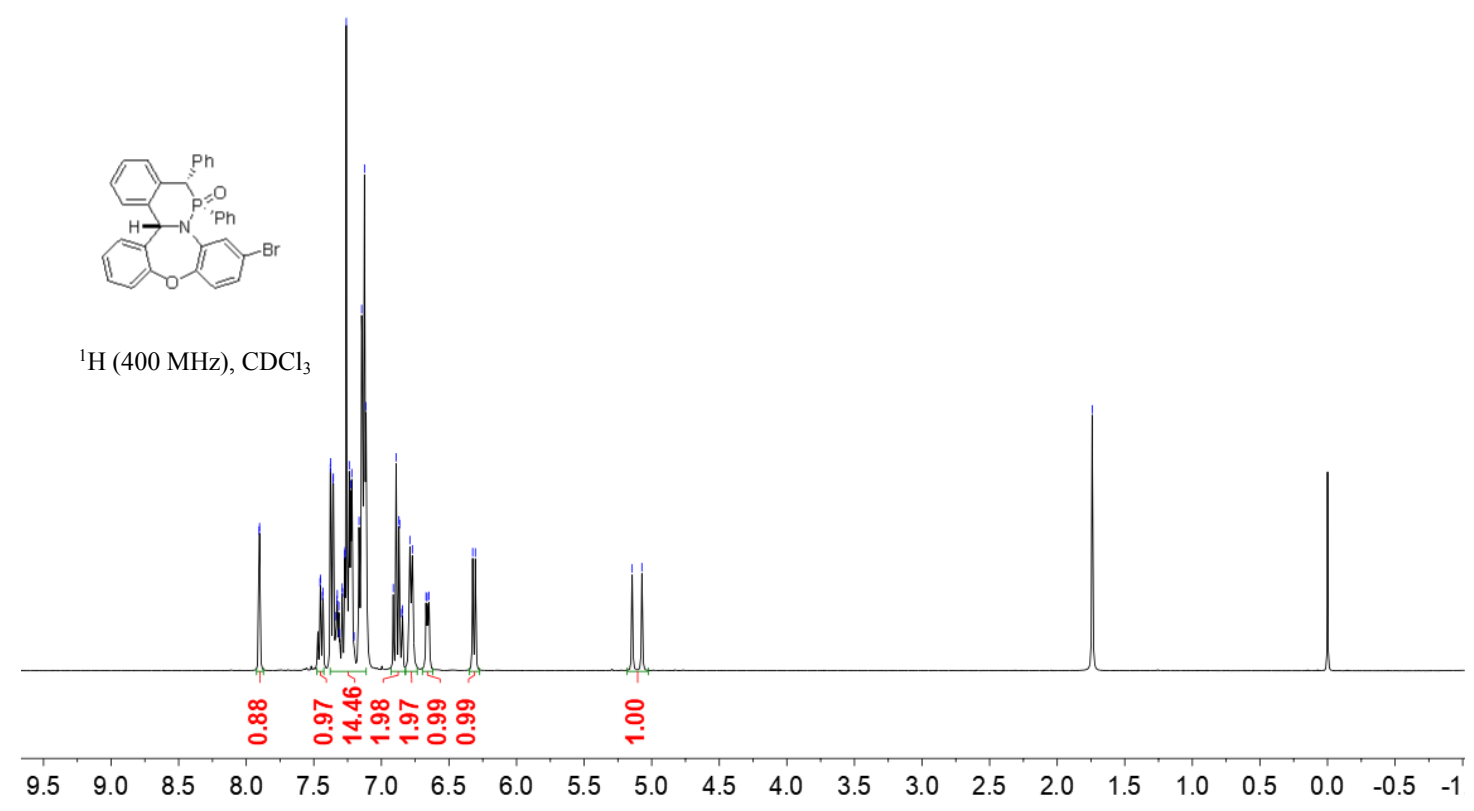

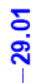

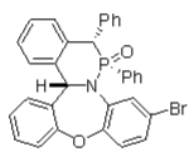

${ }^{31} \mathrm{P}(162 \mathrm{MHz}), \mathrm{CDCl}_{3}$

\begin{tabular}{lllllllllllllllll}
\hline 0 & 80 & 60 & 40 & 20 & 0 & -20 & -40 & -60 & -80 & -100 & -120 & -140 & -160 & -180 & -20
\end{tabular}




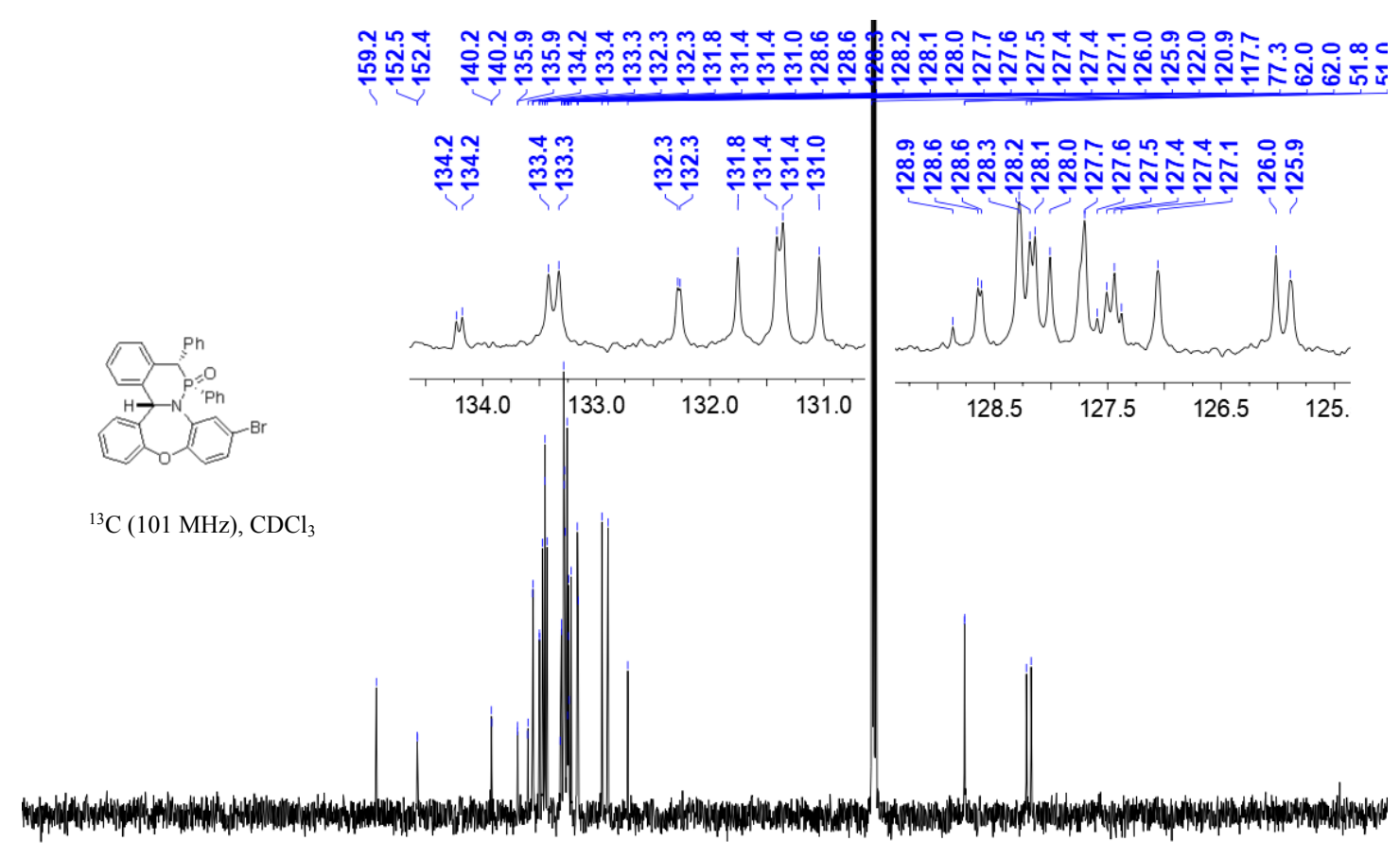

$\begin{array}{llllllllllllllllllllll}210 & 200 & 190 & 180 & 170 & 160 & 150 & 140 & 130 & 120 & 110 & 100 & 90 & 80 & 70 & 60 & 50 & 40 & 30 & 20 & 10 & 0\end{array}$

${ }^{1} \mathrm{H}(400 \mathrm{MHz}),{ }^{31} \mathrm{P}(162 \mathrm{MHz})$ and ${ }^{13} \mathrm{C}(101 \mathrm{MHz})$ spectra of compound $\mathbf{3 g}$

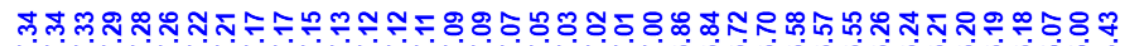

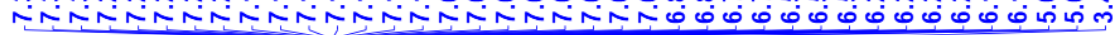

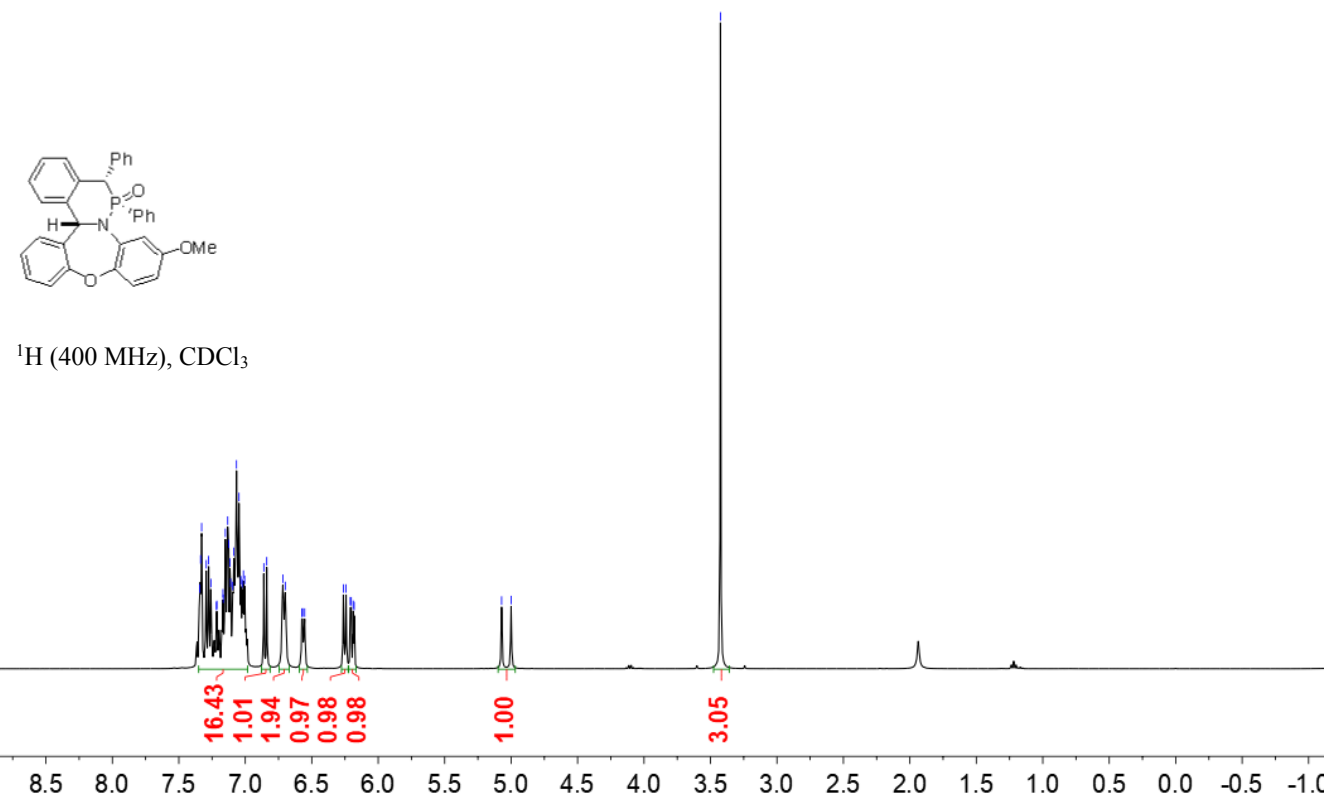




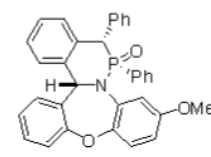

${ }^{31} \mathrm{P}(162 \mathrm{MHz}), \mathrm{CDCl}_{3}$

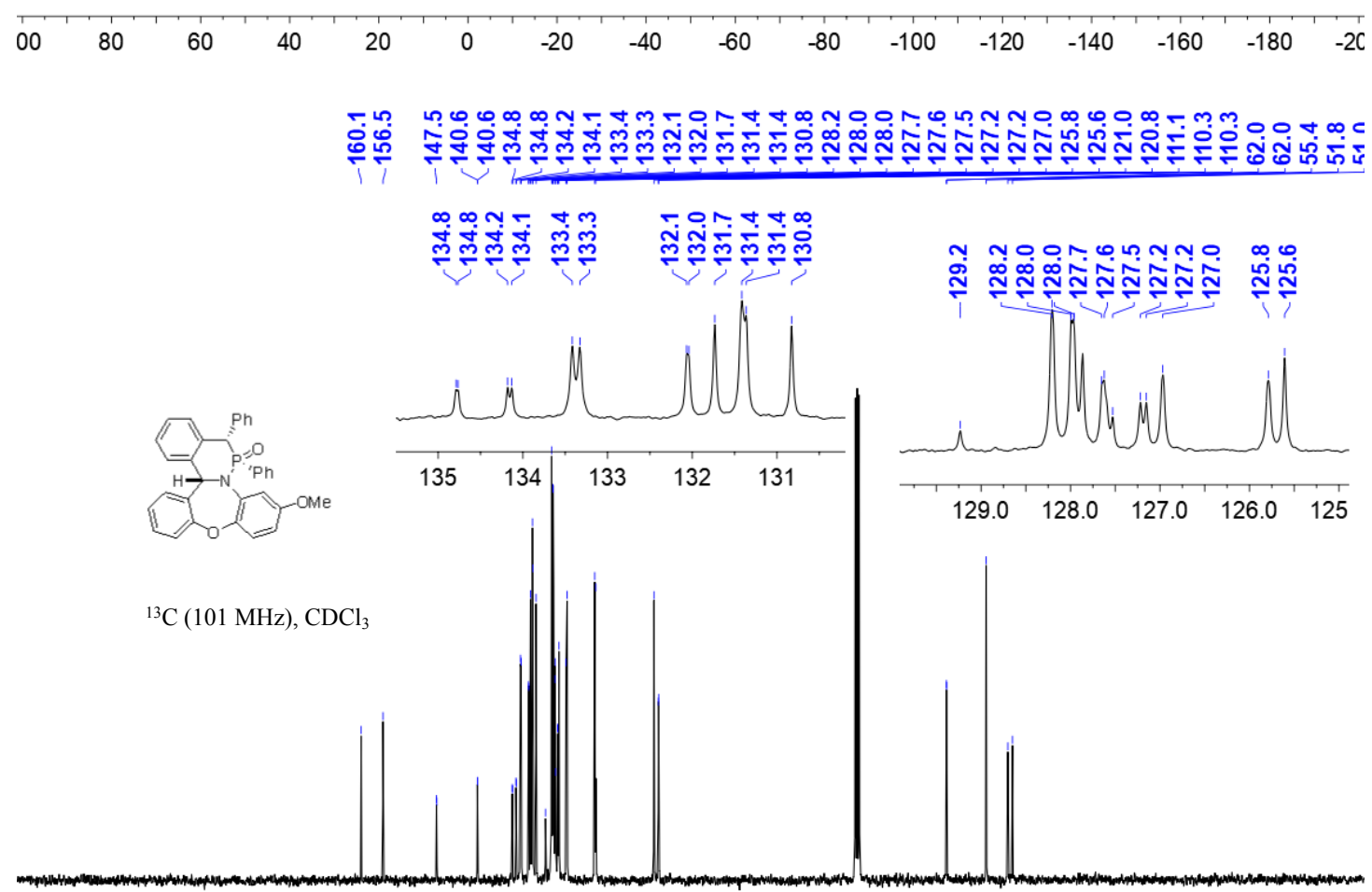

$\begin{array}{llllllllllllllllllllll}210 & 200 & 190 & 180 & 170 & 160 & 150 & 140 & 130 & 120 & 110 & 100 & 90 & 80 & 70 & 60 & 50 & 40 & 30 & 20 & 10 & 0\end{array}$

${ }^{1} \mathrm{H}(400 \mathrm{MHz}),{ }^{31} \mathrm{P}(162 \mathrm{MHz})$ and ${ }^{13} \mathrm{C}(101 \mathrm{MHz})$ spectra of compound $\mathbf{3 h}$ 


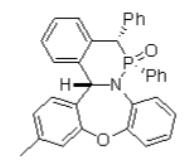

${ }^{1} \mathrm{H}(400 \mathrm{MHz}), \mathrm{CDCl}_{3}$

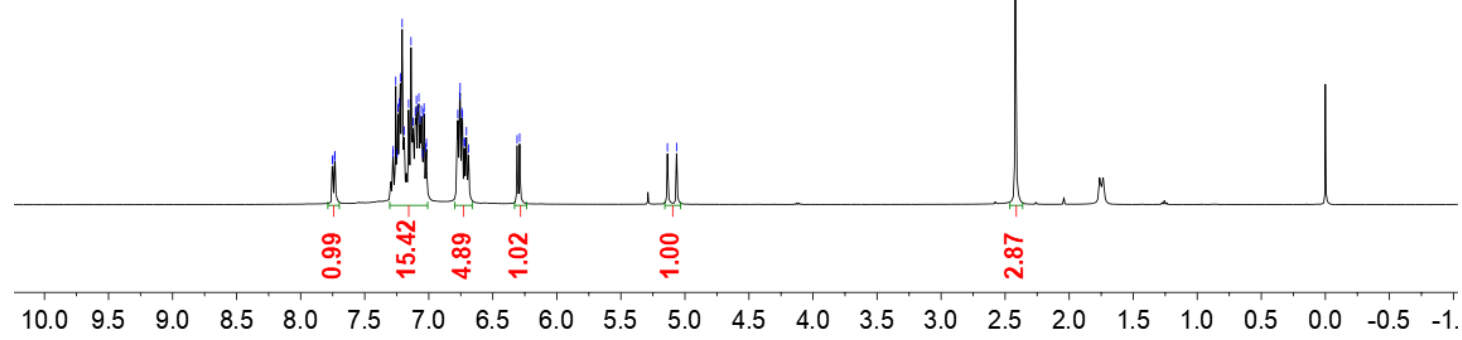

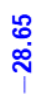

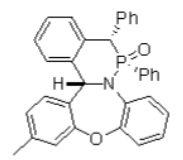

${ }^{31} \mathrm{P}(162 \mathrm{MHz}), \mathrm{CDCl}_{3}$

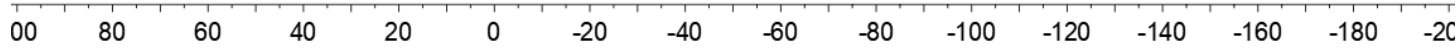




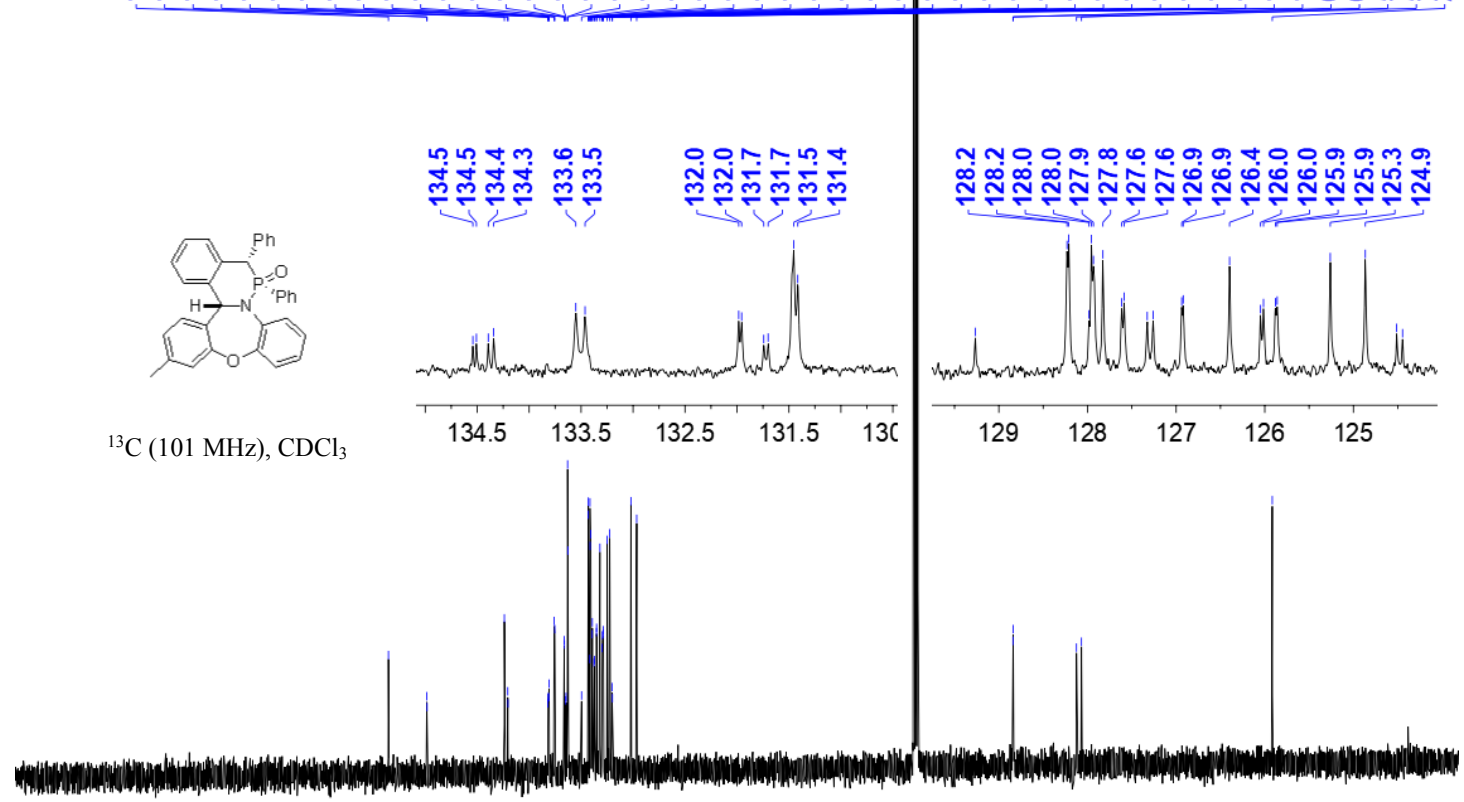

$\begin{array}{llllllllllllllllllllll}210 & 200 & 190 & 180 & 170 & 160 & 150 & 140 & 130 & 120 & 110 & 100 & 90 & 80 & 70 & 60 & 50 & 40 & 30 & 20 & 10 & 0\end{array}$

${ }^{1} \mathrm{H}(400 \mathrm{MHz}),{ }^{31} \mathrm{P}(162 \mathrm{MHz})$ and ${ }^{13} \mathrm{C}(101 \mathrm{MHz})$ spectra of compound $3 \mathbf{i}$

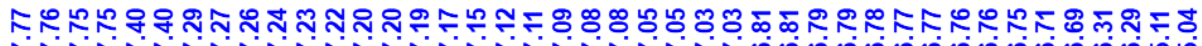

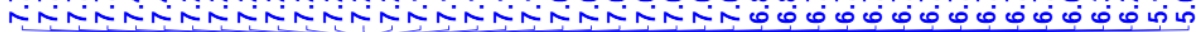

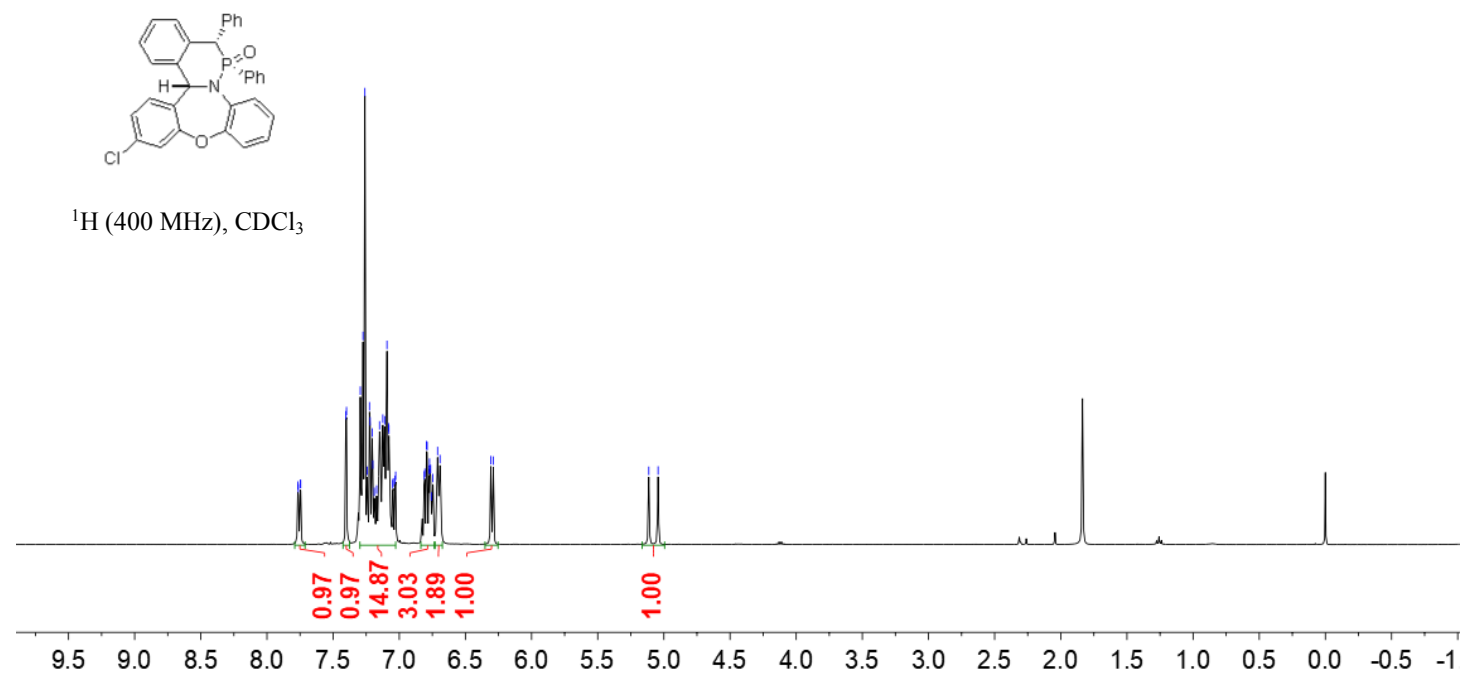




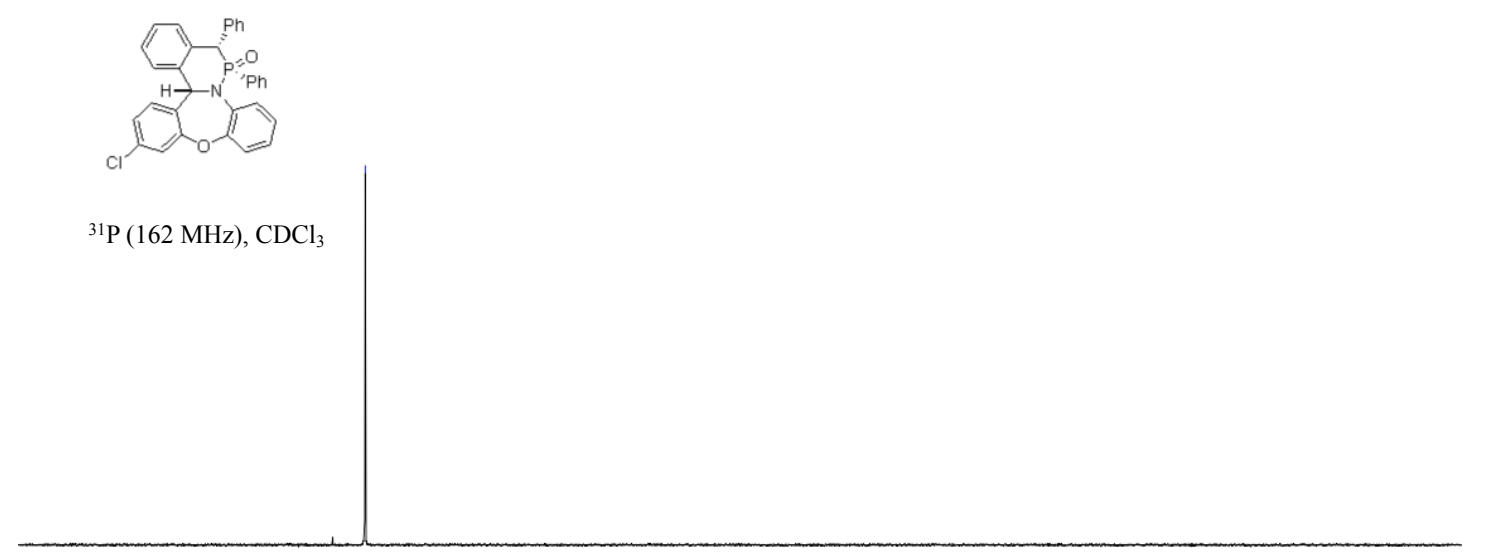

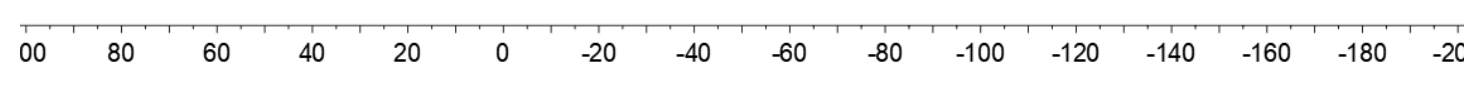

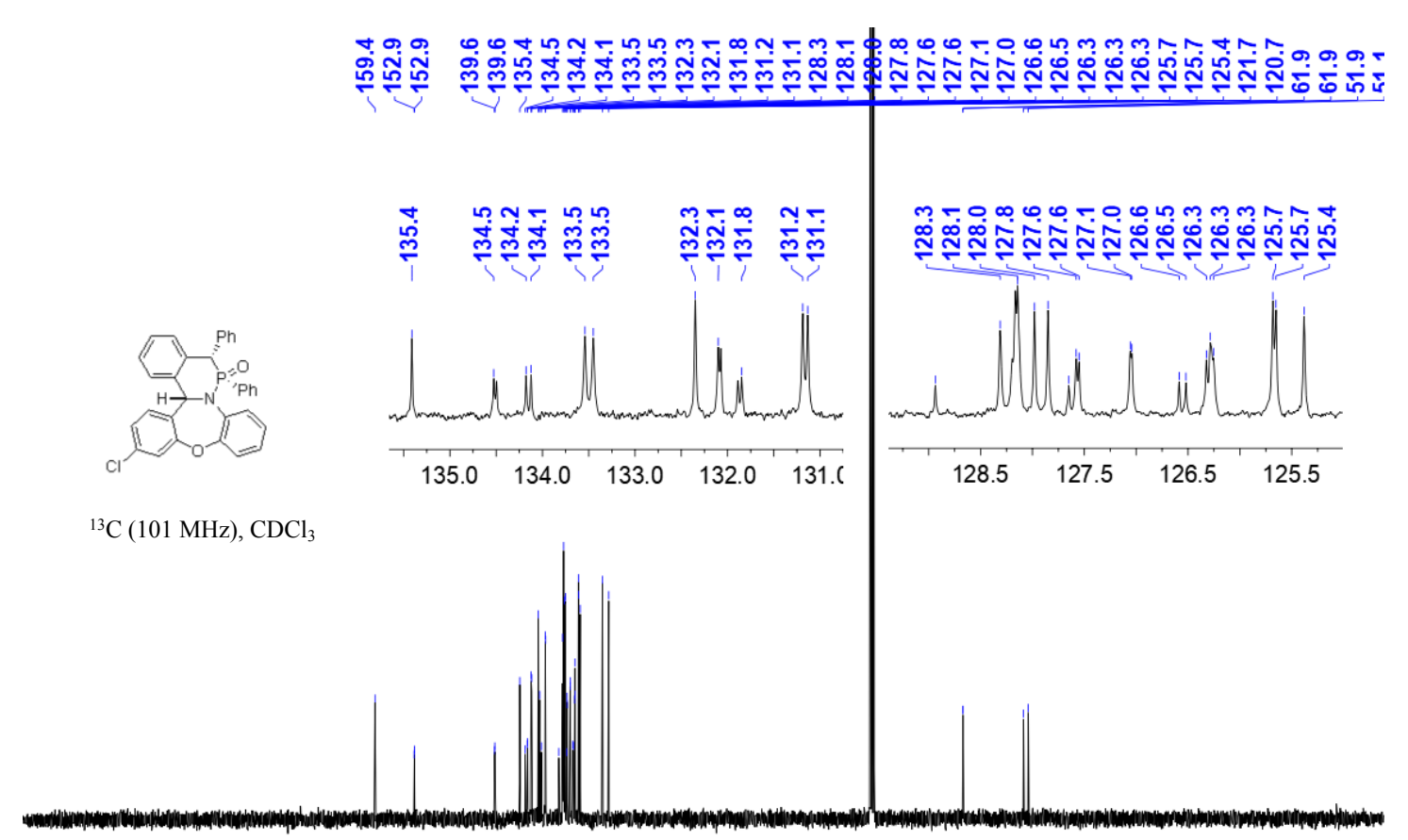

$\begin{array}{llllllllllllllllllllll}210 & 200 & 190 & 180 & 170 & 160 & 150 & 140 & 130 & 120 & 110 & 100 & 90 & 80 & 70 & 60 & 50 & 40 & 30 & 20 & 10 & 0\end{array}$

${ }^{1} \mathrm{H}(400 \mathrm{MHz}),{ }^{31} \mathrm{P}(162 \mathrm{MHz})$ and ${ }^{13} \mathrm{C}(101 \mathrm{MHz})$ spectra of compound $\mathbf{3 j}$ 


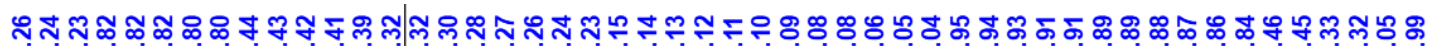

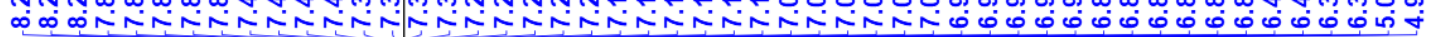

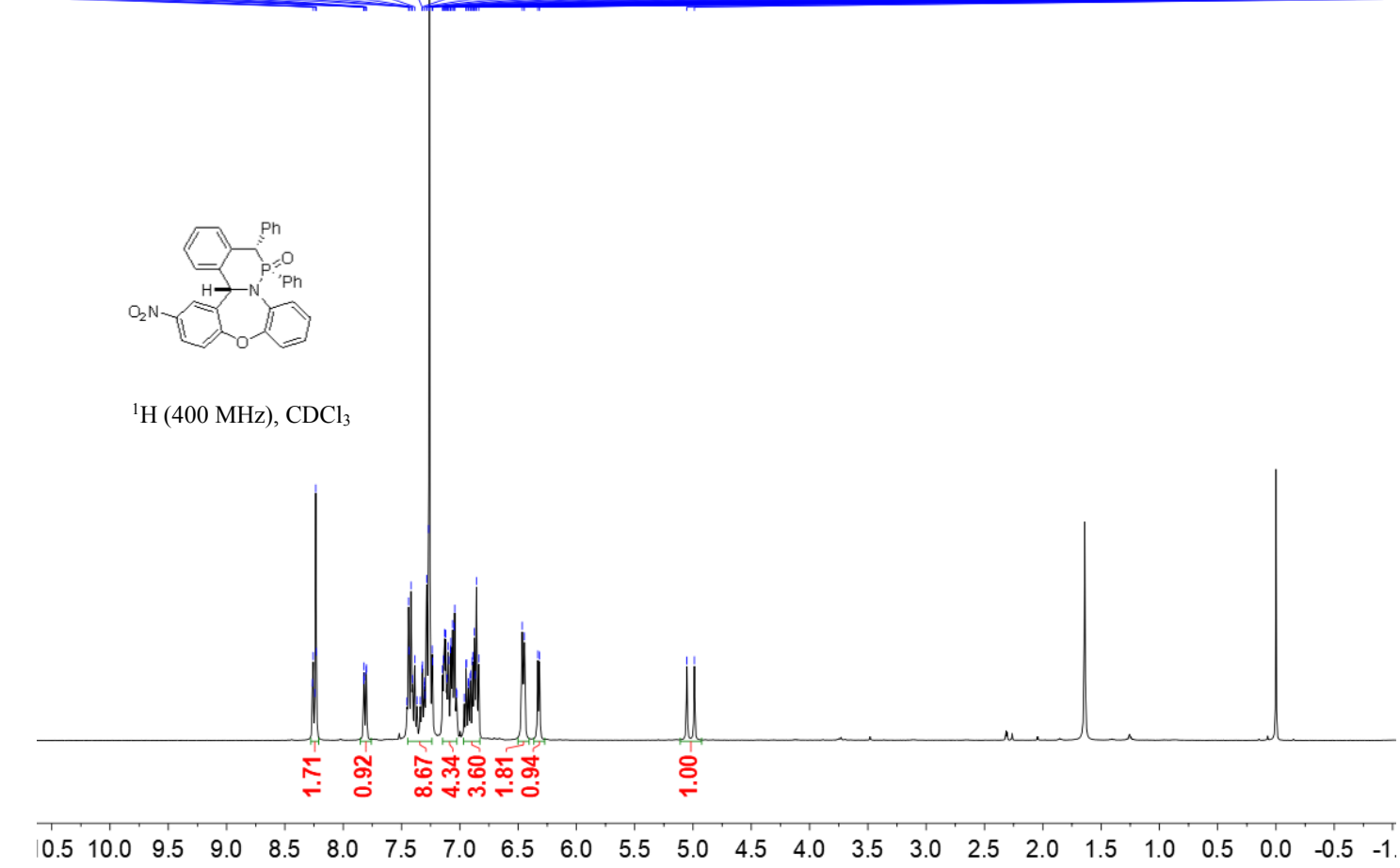

$\underset{\sim}{\sim}$

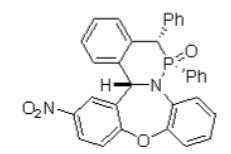

${ }^{31} \mathrm{P}(162 \mathrm{MHz}), \mathrm{CDCl}_{3}$

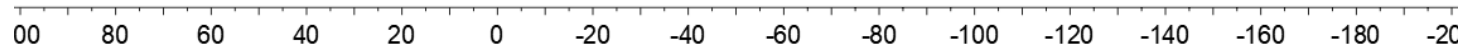




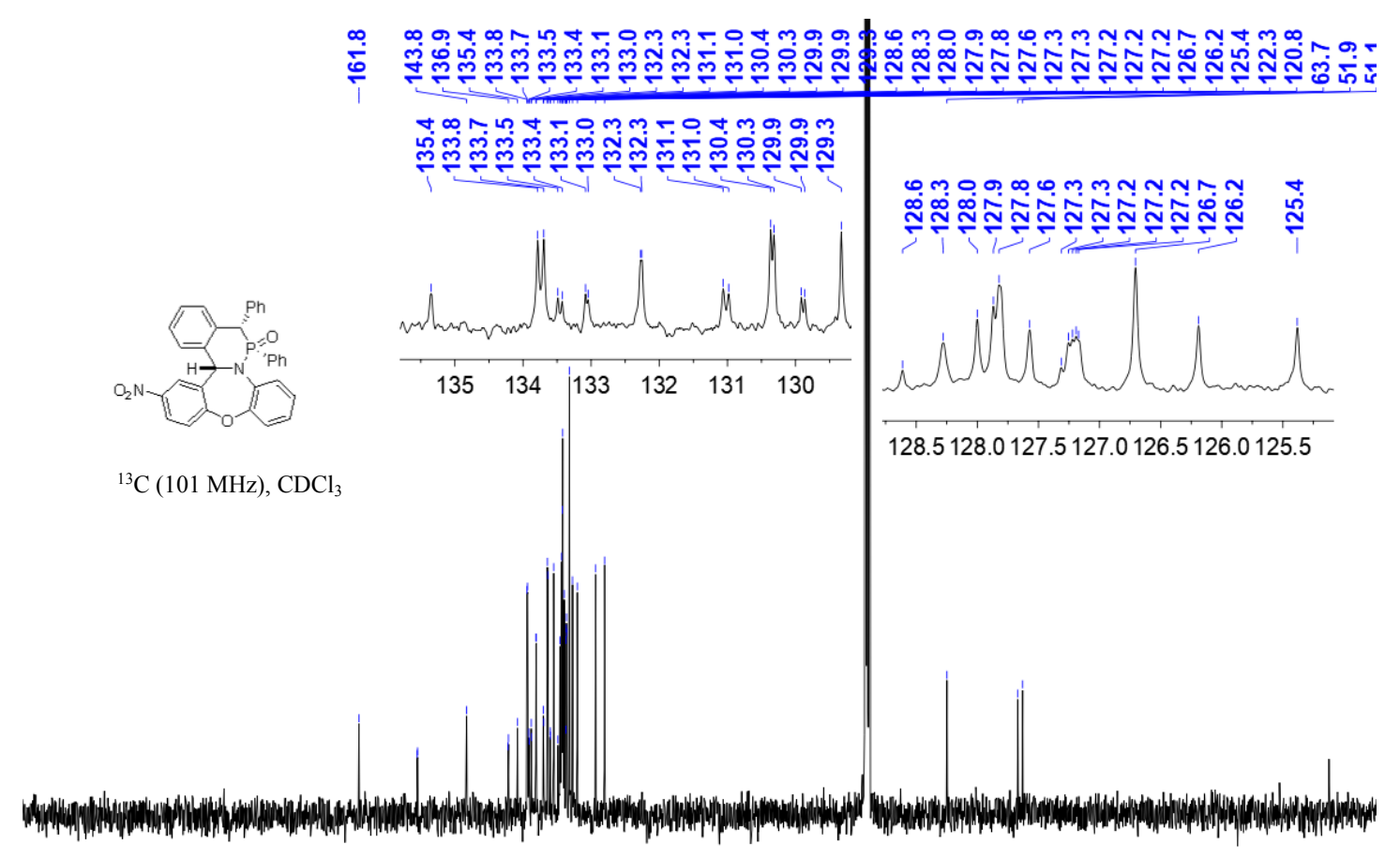

$\begin{array}{llllllllllllllllllllll}210 & 200 & 190 & 180 & 170 & 160 & 150 & 140 & 130 & 120 & 110 & 100 & 90 & 80 & 70 & 60 & 50 & 40 & 30 & 20 & 10 & 0\end{array}$

${ }^{1} \mathrm{H}(400 \mathrm{MHz}),{ }^{31} \mathrm{P}(162 \mathrm{MHz})$ and ${ }^{13} \mathrm{C}(101 \mathrm{MHz})$ spectra of compound 3k

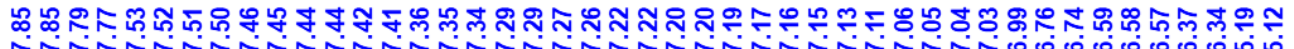

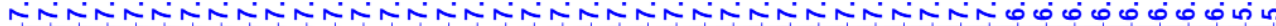
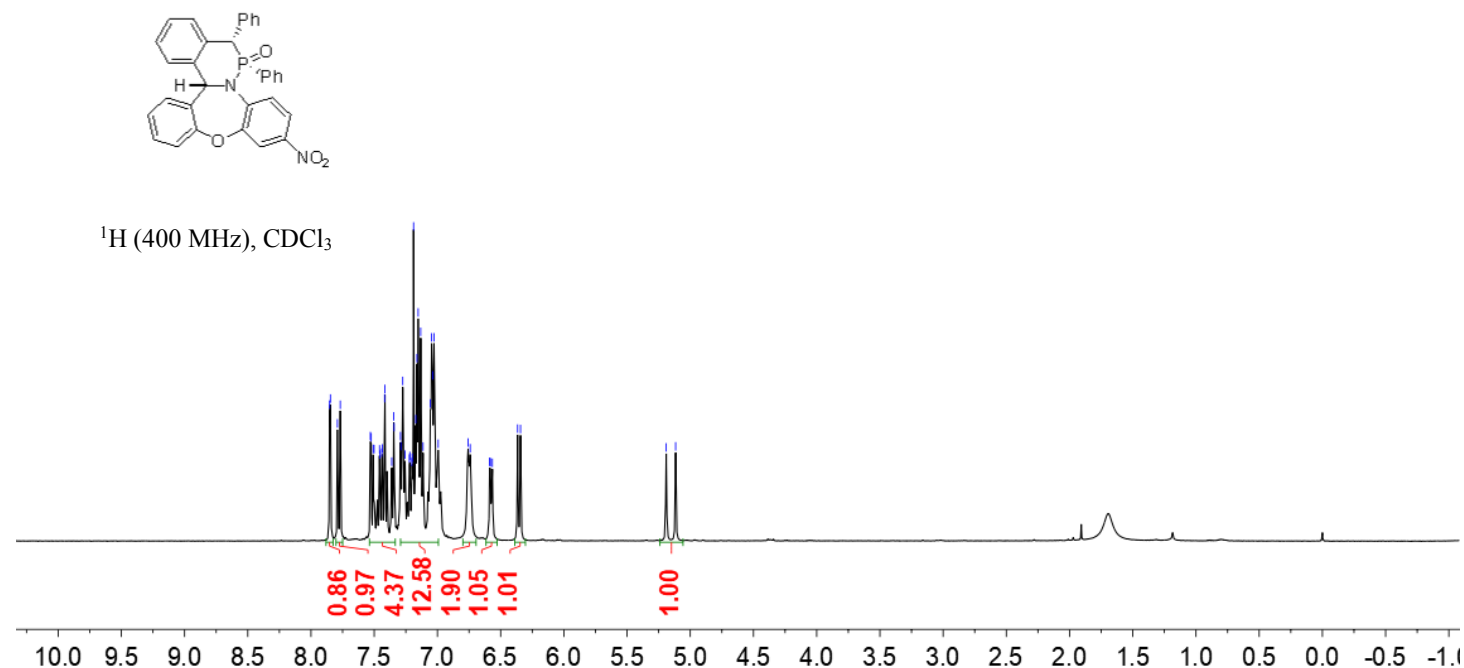


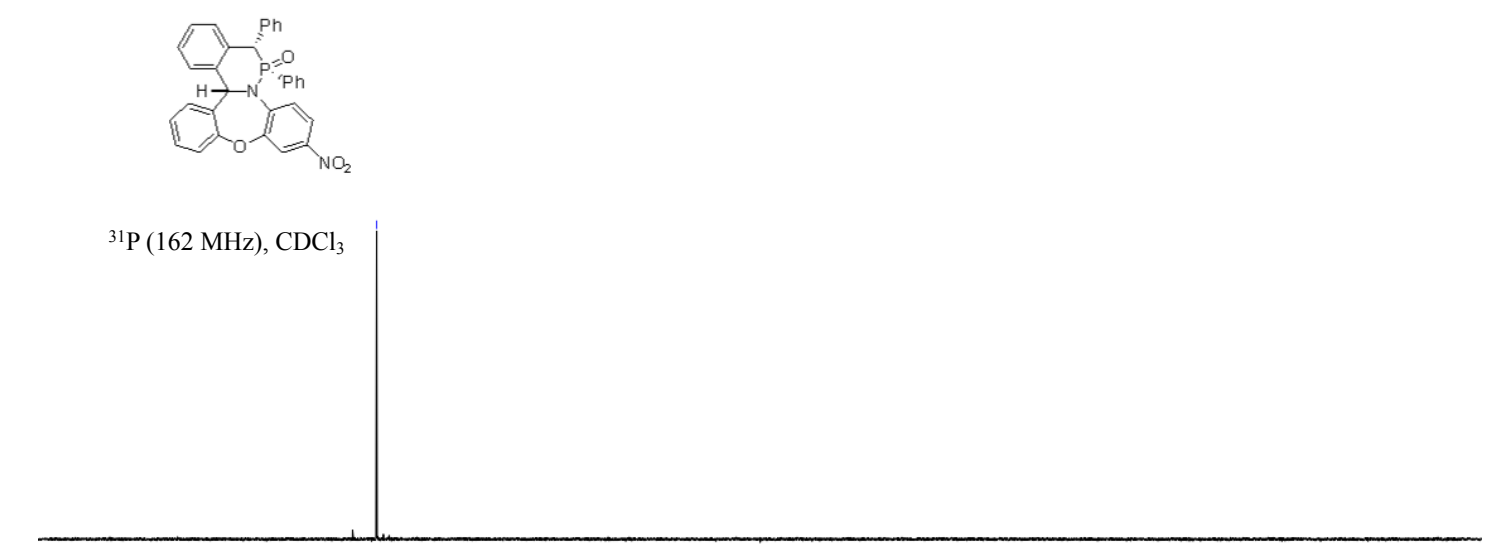

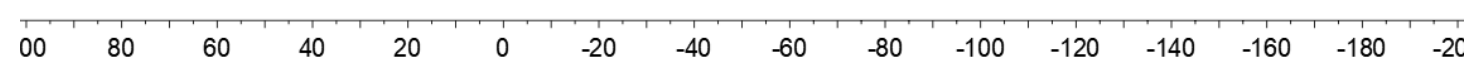

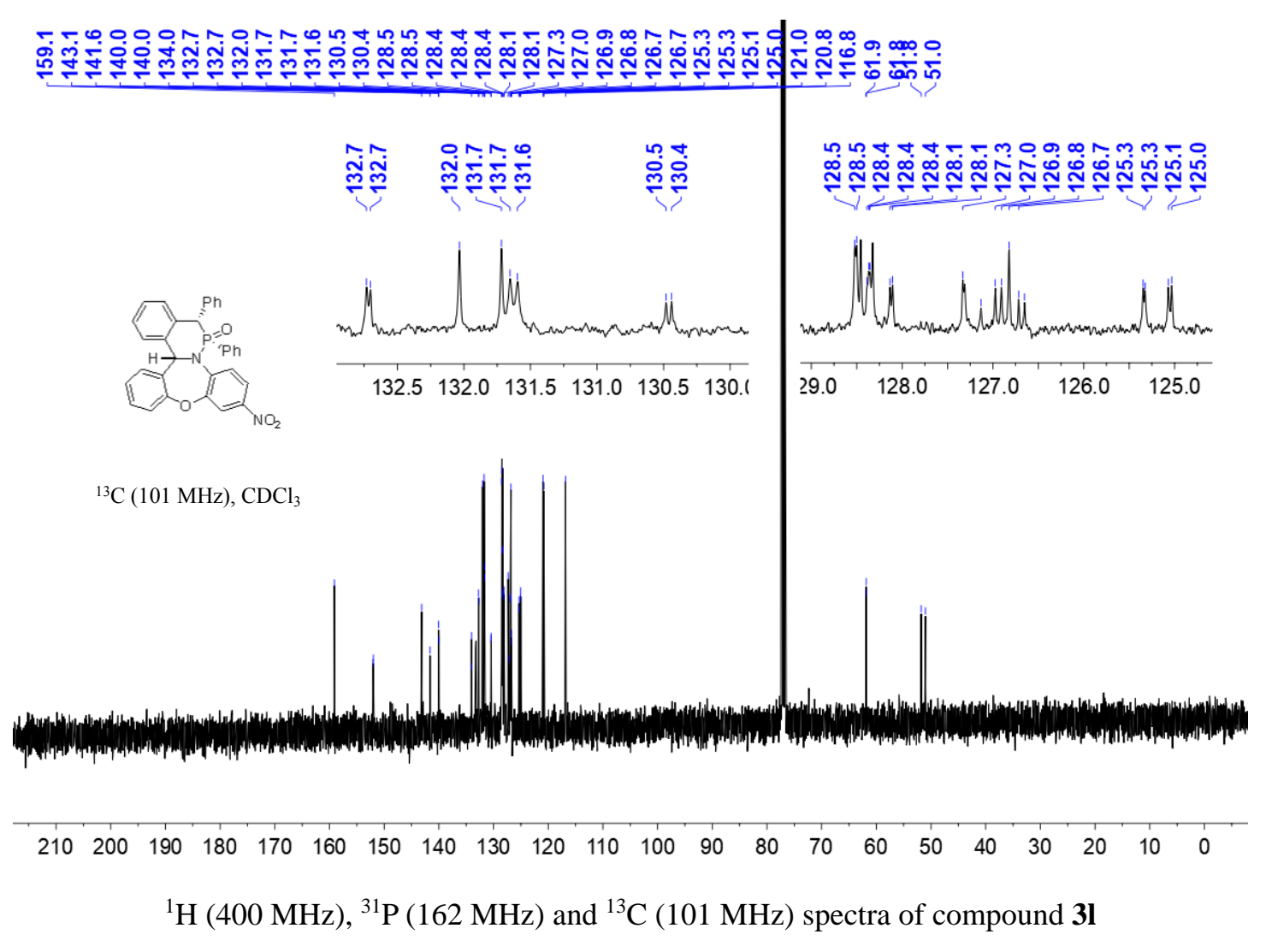




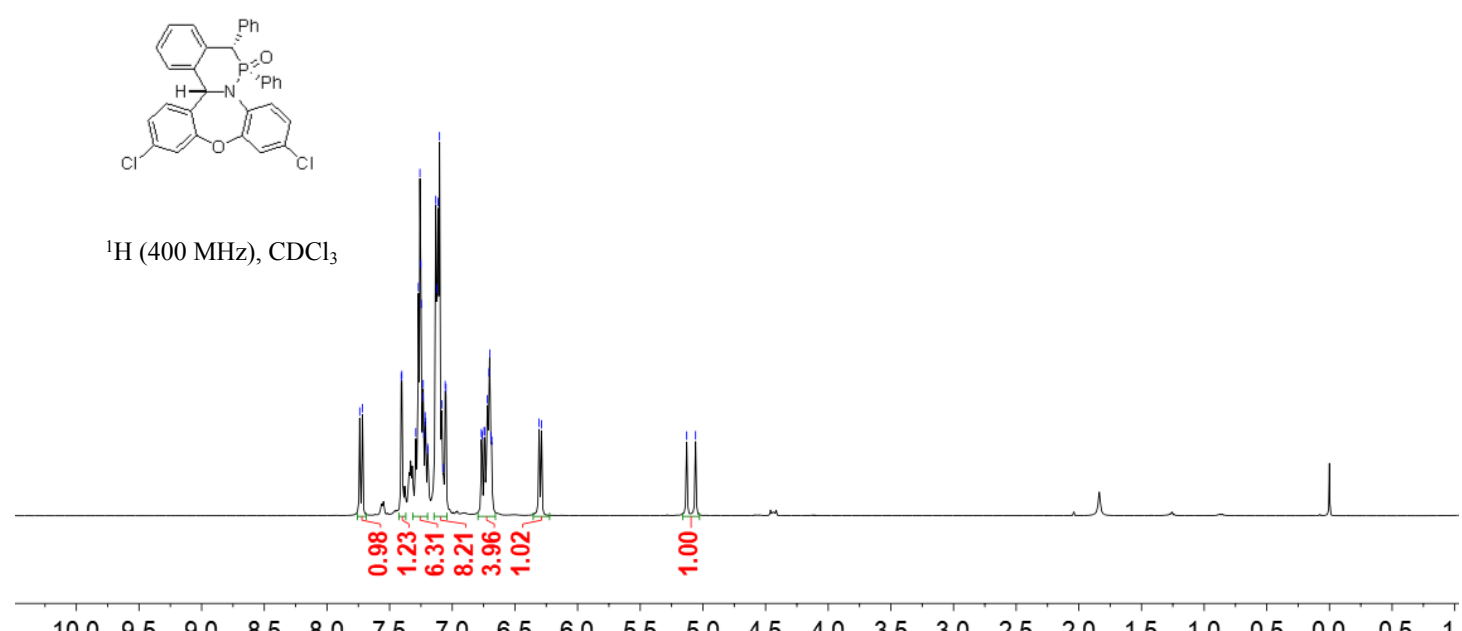

ஸ్

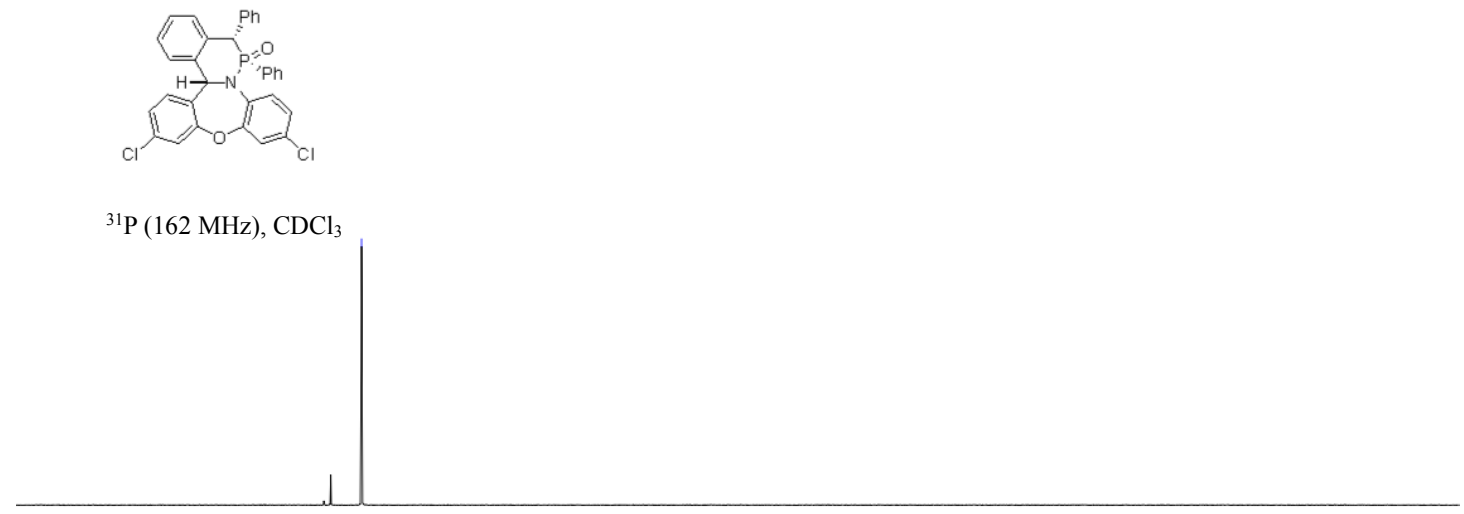

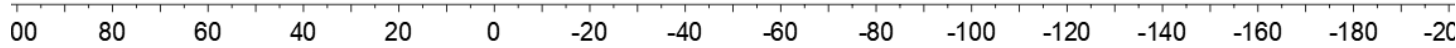



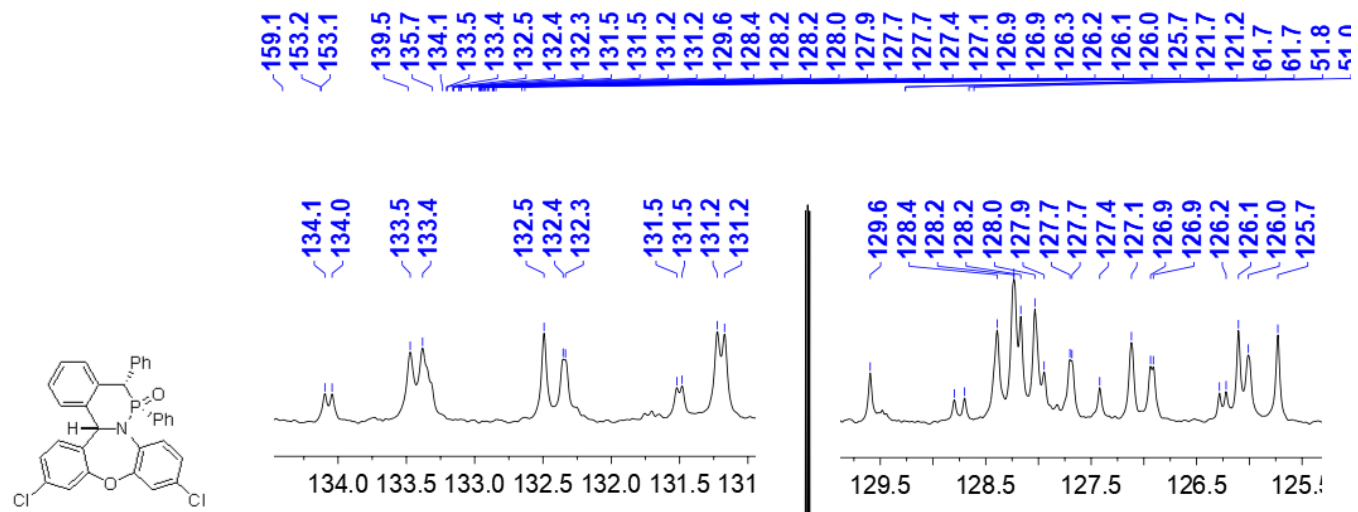

${ }^{13} \mathrm{C}(101 \mathrm{MHz}), \mathrm{CDCl}_{3}$

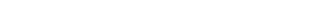

$\begin{array}{llllllllllllllllllllll}210 & 200 & 190 & 180 & 170 & 160 & 150 & 140 & 130 & 120 & 110 & 100 & 90 & 80 & 70 & 60 & 50 & 40 & 30 & 20 & 10 & 0\end{array}$

${ }^{1} \mathrm{H}(400 \mathrm{MHz}),{ }^{31} \mathrm{P}(162 \mathrm{MHz})$ and ${ }^{13} \mathrm{C}(101 \mathrm{MHz})$ spectra of compound $3 \mathbf{m}$

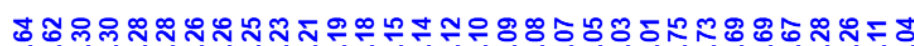

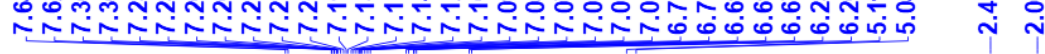

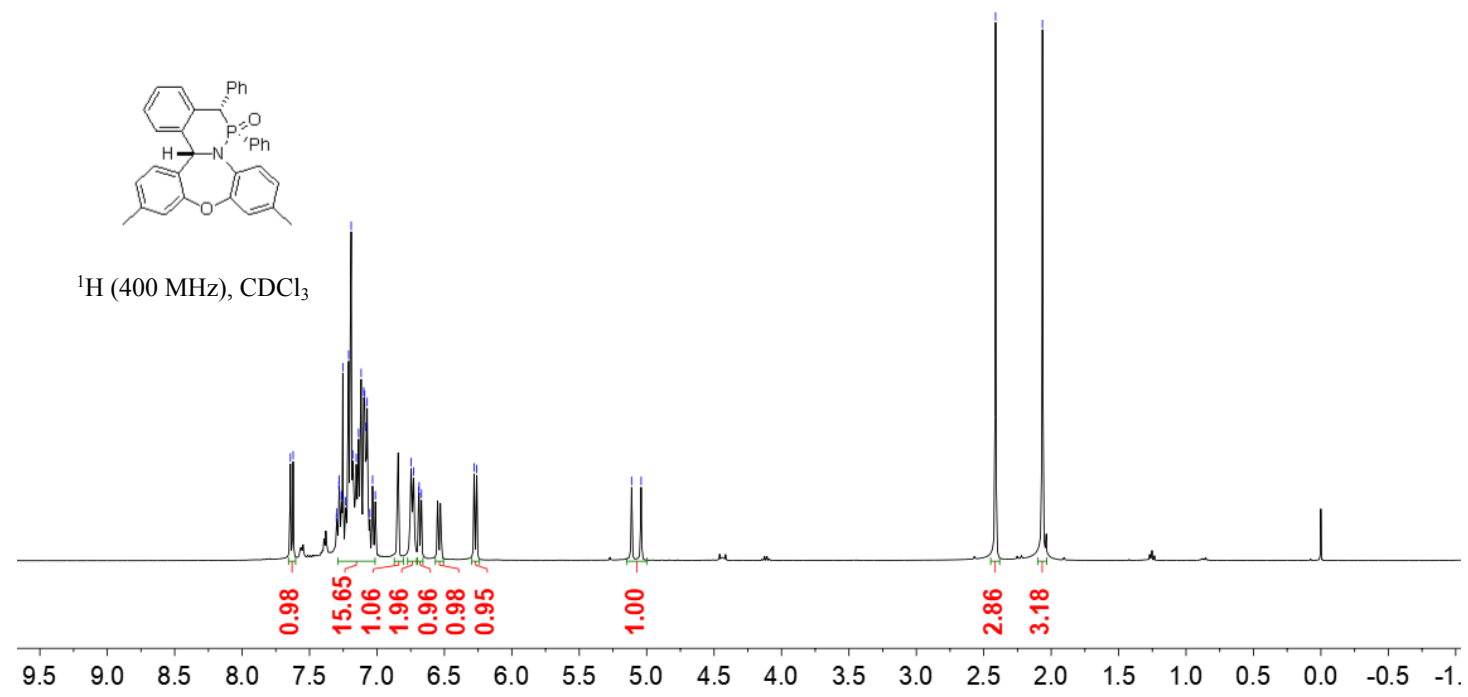




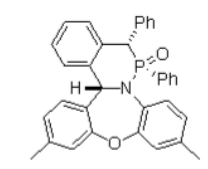

${ }^{31} \mathrm{P}(162 \mathrm{MHz}), \mathrm{CDCl}_{3}$

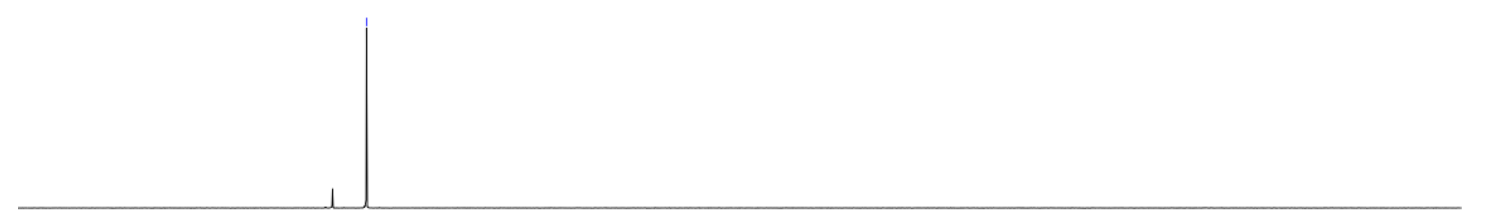

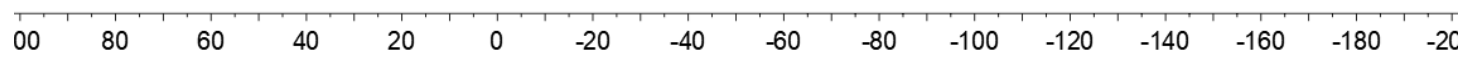
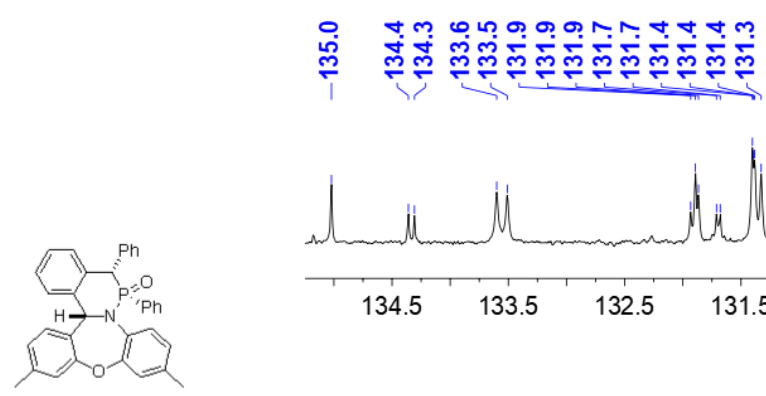

în.
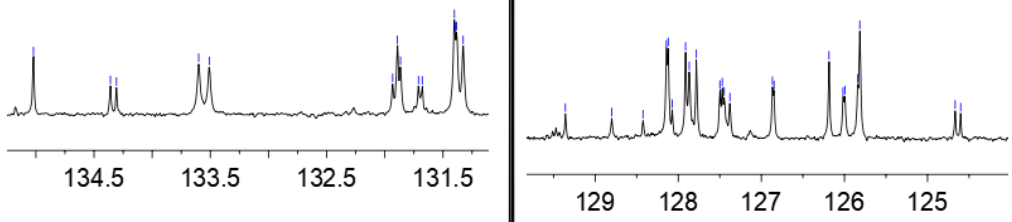

${ }^{13} \mathrm{C}(101 \mathrm{MHz}), \mathrm{CDCl}_{3}$

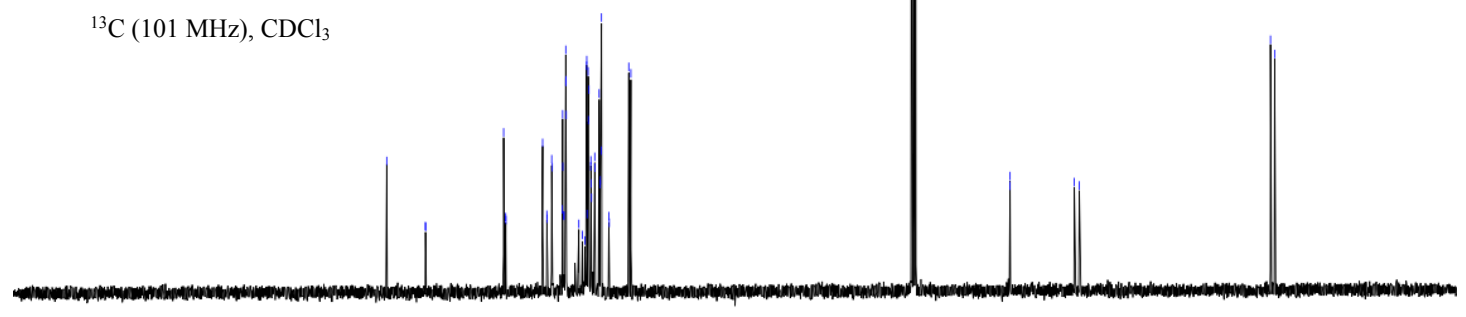

$\begin{array}{llllllllllllllllllllll}210 & 200 & 190 & 180 & 170 & 160 & 150 & 140 & 130 & 120 & 110 & 100 & 90 & 80 & 70 & 60 & 50 & 40 & 30 & 20 & 10 & 0\end{array}$

${ }^{1} \mathrm{H}(400 \mathrm{MHz}),{ }^{31} \mathrm{P}(162 \mathrm{MHz})$ and ${ }^{13} \mathrm{C}(101 \mathrm{MHz})$ spectra of compound $\mathbf{3 n}$ 


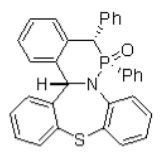

${ }^{1} \mathrm{H}(400 \mathrm{MHz}), \mathrm{CDCl}_{3}$

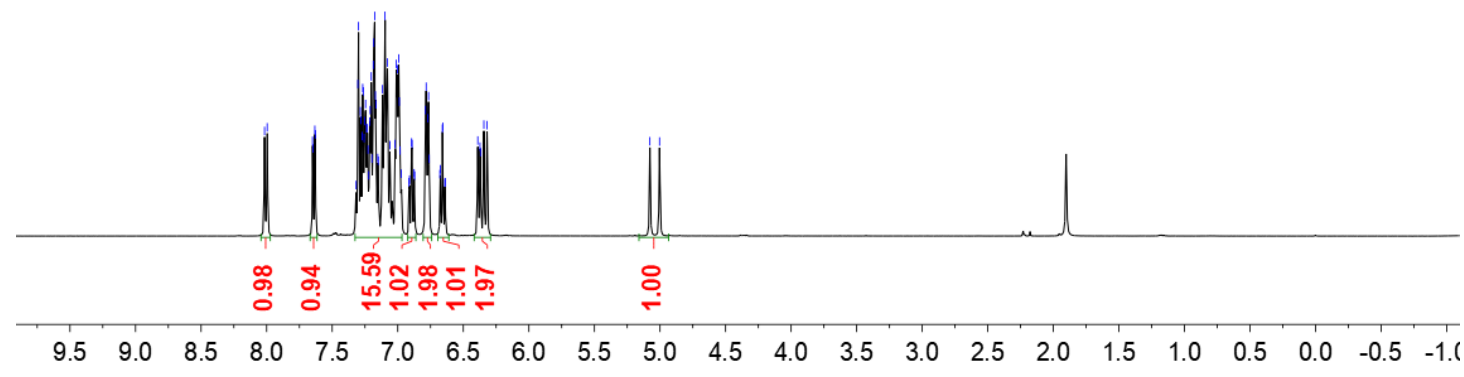

ণ্ণ

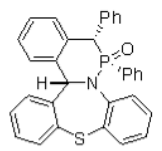

${ }^{31} \mathrm{P}(162 \mathrm{MHz}), \mathrm{CDCl}_{3}$

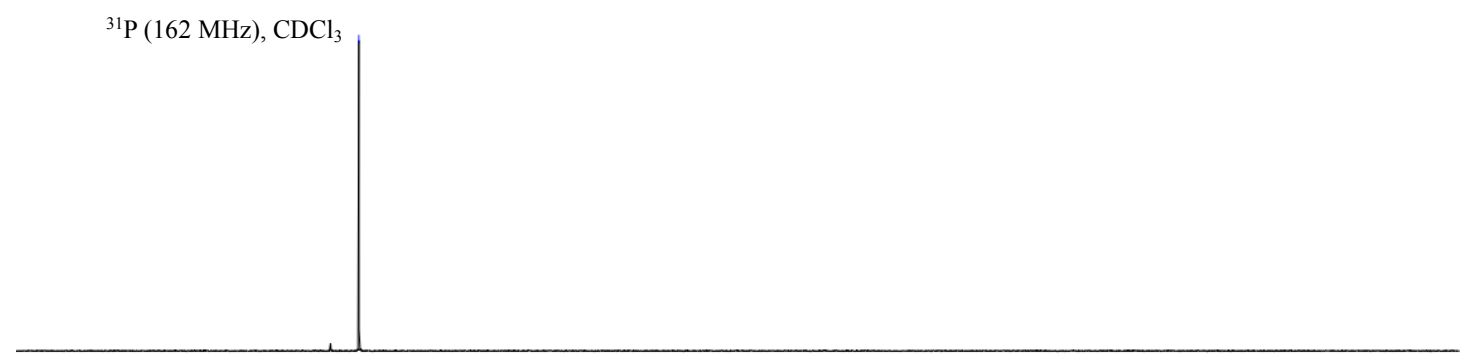

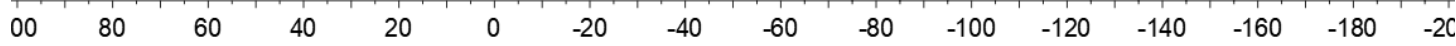




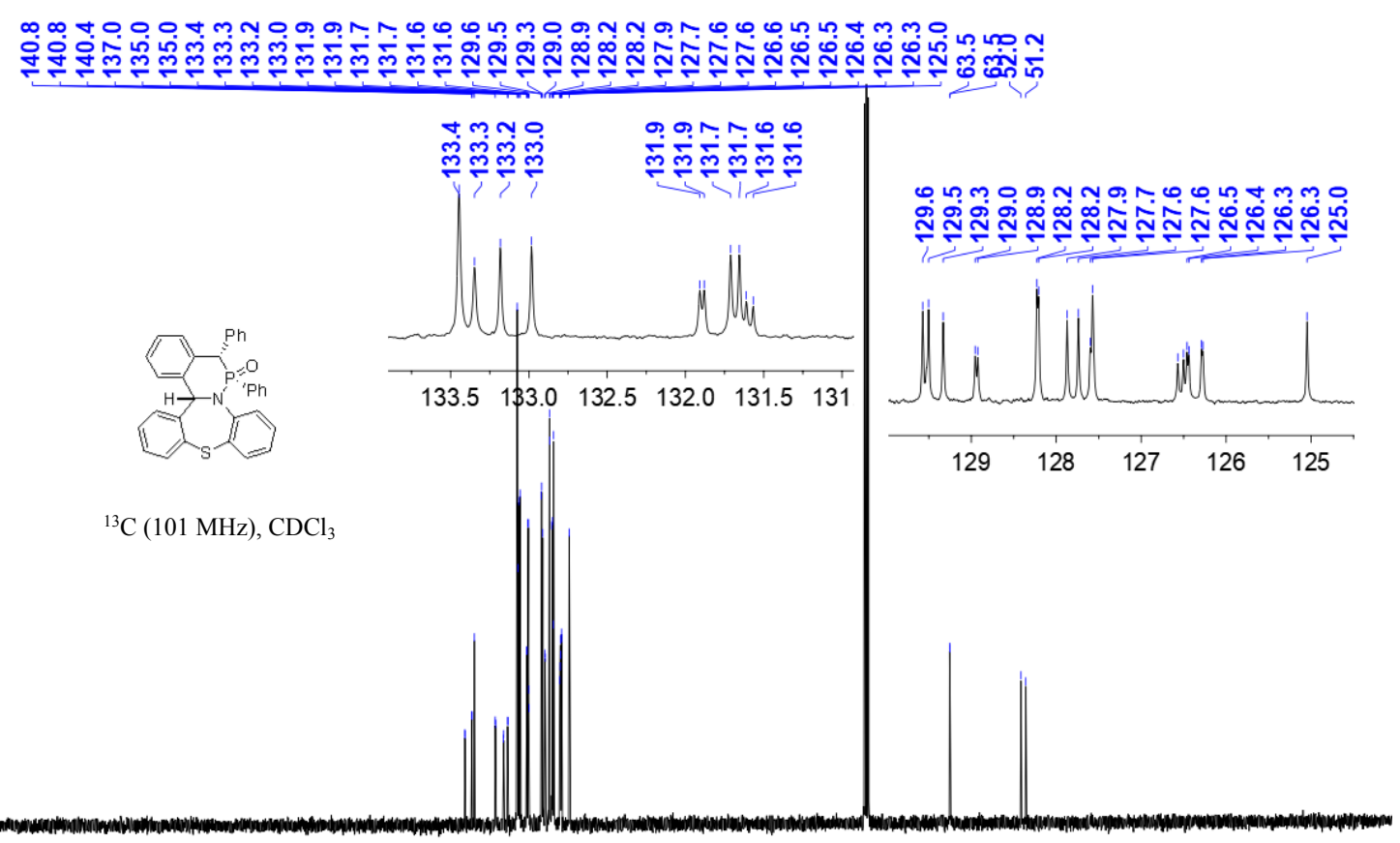

$\begin{array}{llllllllllllllllllllll}210 & 200 & 190 & 180 & 170 & 160 & 150 & 140 & 130 & 120 & 110 & 100 & 90 & 80 & 70 & 60 & 50 & 40 & 30 & 20 & 10 & 0\end{array}$

${ }^{1} \mathrm{H}(400 \mathrm{MHz}),{ }^{31} \mathrm{P}(162 \mathrm{MHz})$ and ${ }^{13} \mathrm{C}(101 \mathrm{MHz})$ spectra of compound 3o

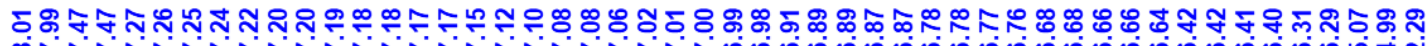

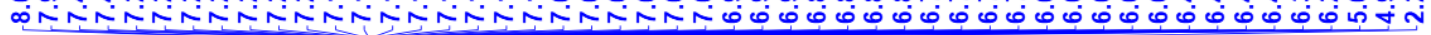

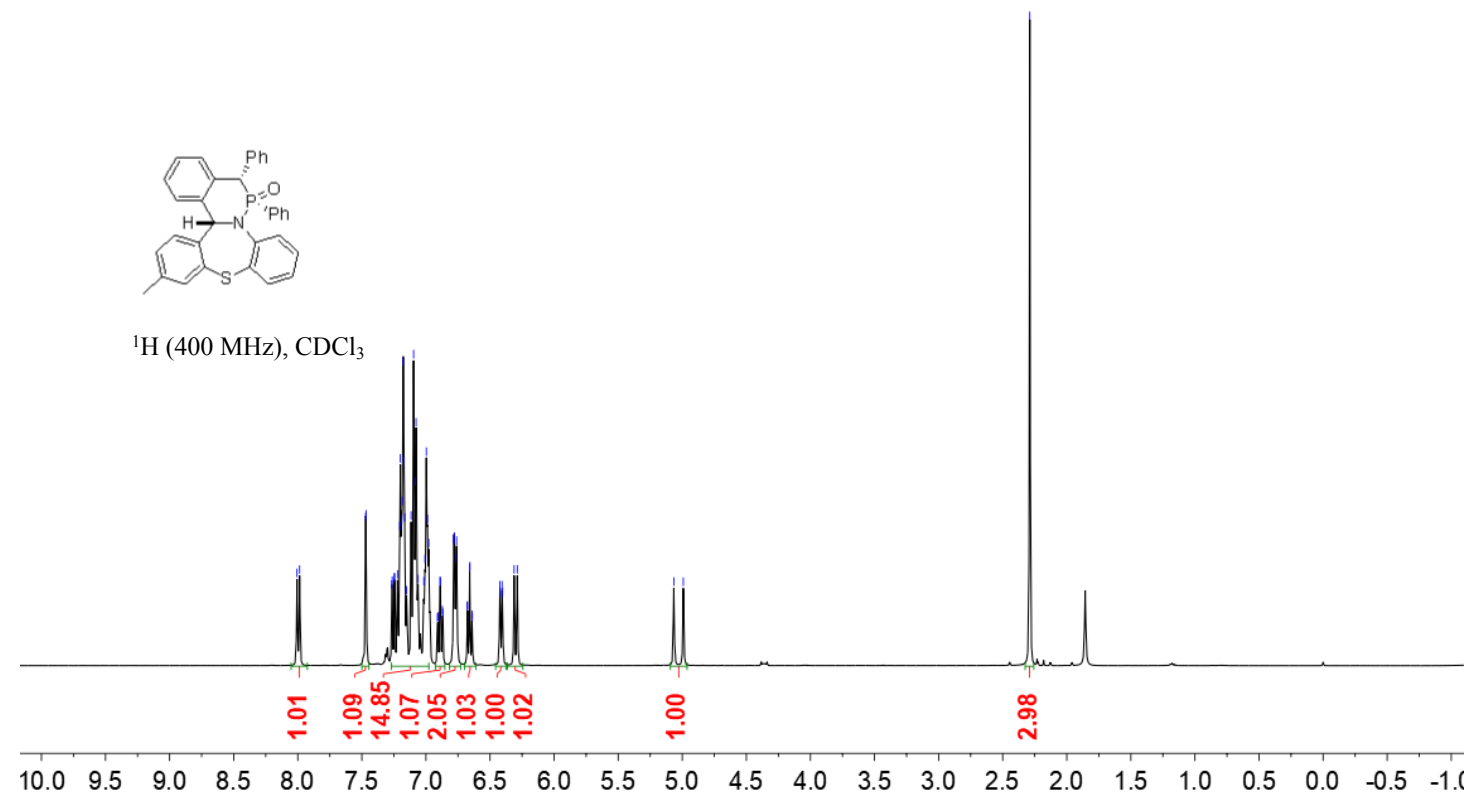




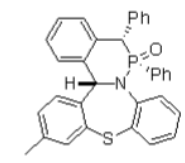

${ }^{31} \mathrm{P}(162 \mathrm{MHz}), \mathrm{CDCl}_{3}$

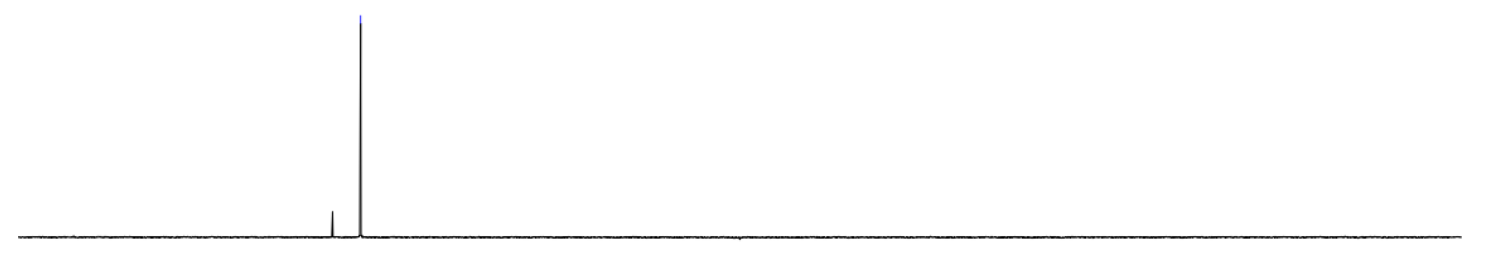

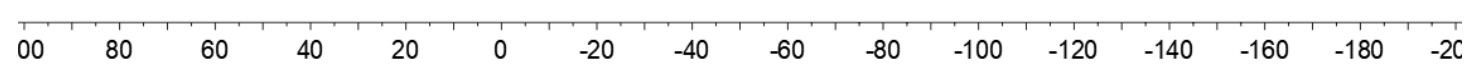

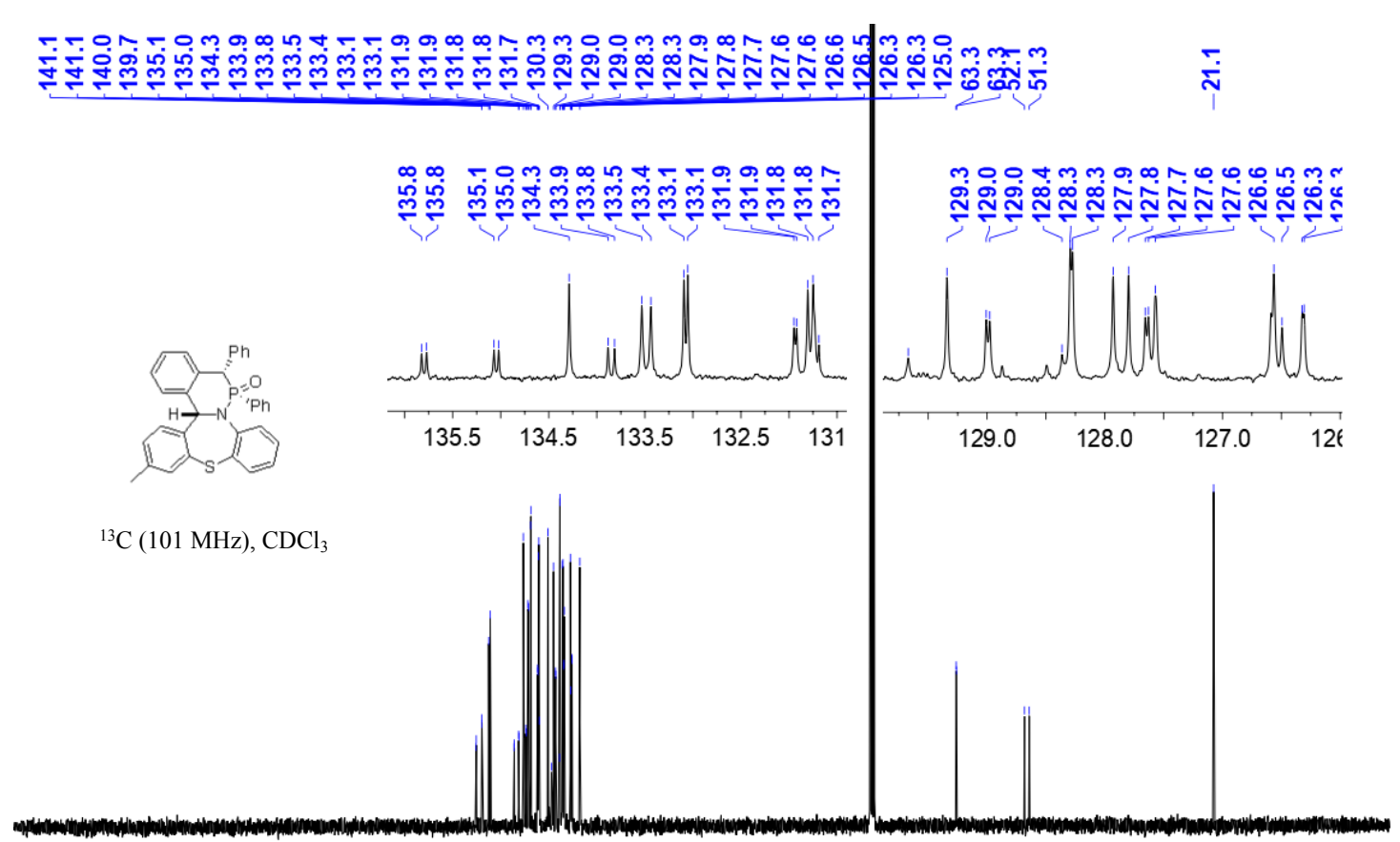

$\begin{array}{llllllllllllllllllllll}210 & 200 & 190 & 180 & 170 & 160 & 150 & 140 & 130 & 120 & 110 & 100 & 90 & 80 & 70 & 60 & 50 & 40 & 30 & 20 & 10 & 0\end{array}$

${ }^{1} \mathrm{H}(400 \mathrm{MHz}),{ }^{31} \mathrm{P}(162 \mathrm{MHz})$ and ${ }^{13} \mathrm{C}(101 \mathrm{MHz})$ spectra of compound $\mathbf{3 p}$ 


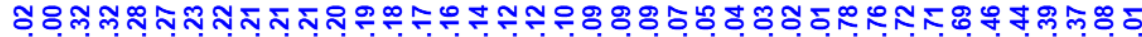

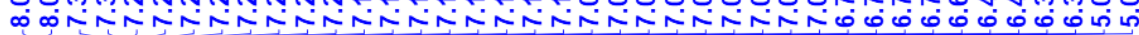

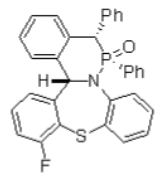

${ }^{1} \mathrm{H}(400 \mathrm{MHz}), \mathrm{CDCl}_{3}$

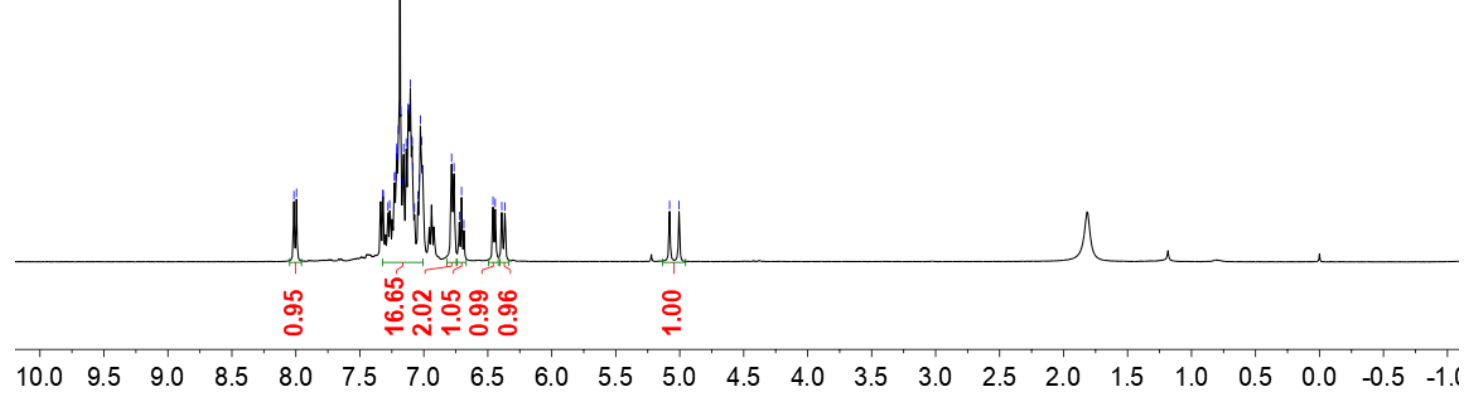

ิั

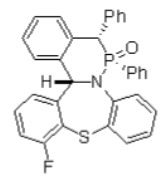

${ }^{31} \mathrm{P}(162 \mathrm{MHz}), \mathrm{CDCl}_{3}$

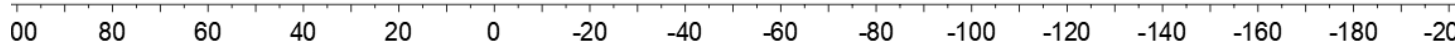




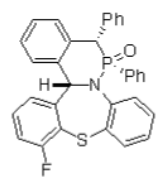

${ }^{19} \mathrm{~F}(376 \mathrm{MHz}), \mathrm{CDCl}_{3}$

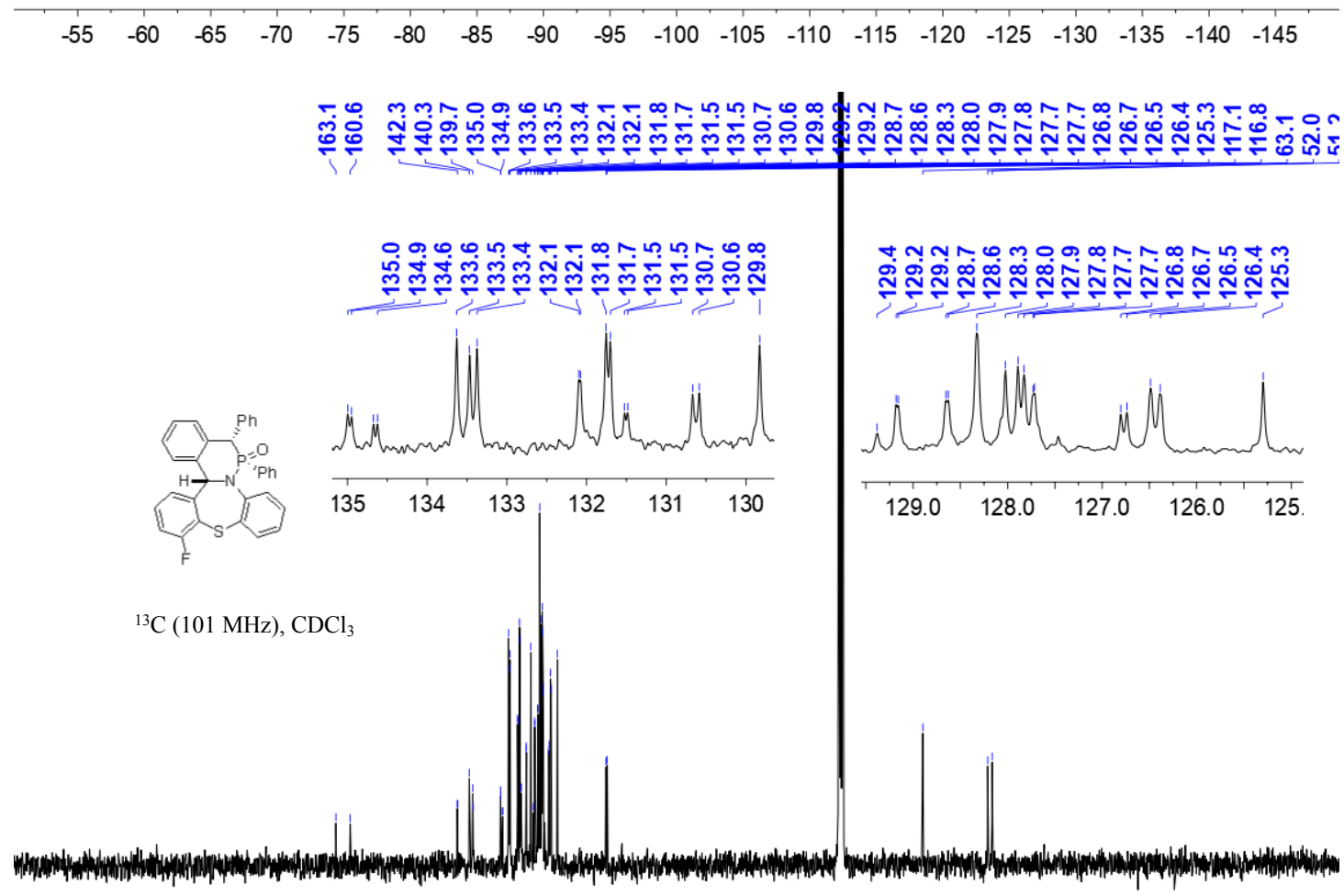

$\begin{array}{llllllllllllllllllllll}210 & 200 & 190 & 180 & 170 & 160 & 150 & 140 & 130 & 120 & 110 & 100 & 90 & 80 & 70 & 60 & 50 & 40 & 30 & 20 & 10 & 0\end{array}$

${ }^{1} \mathrm{H}(400 \mathrm{MHz}),{ }^{31} \mathrm{P}(162 \mathrm{MHz}),{ }^{19} \mathrm{~F} \mathrm{NMR}(376 \mathrm{MHz})$ and ${ }^{13} \mathrm{C}(101 \mathrm{MHz})$ spectra of compound 3q 


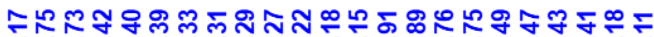

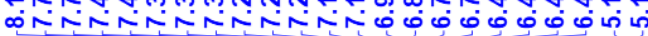
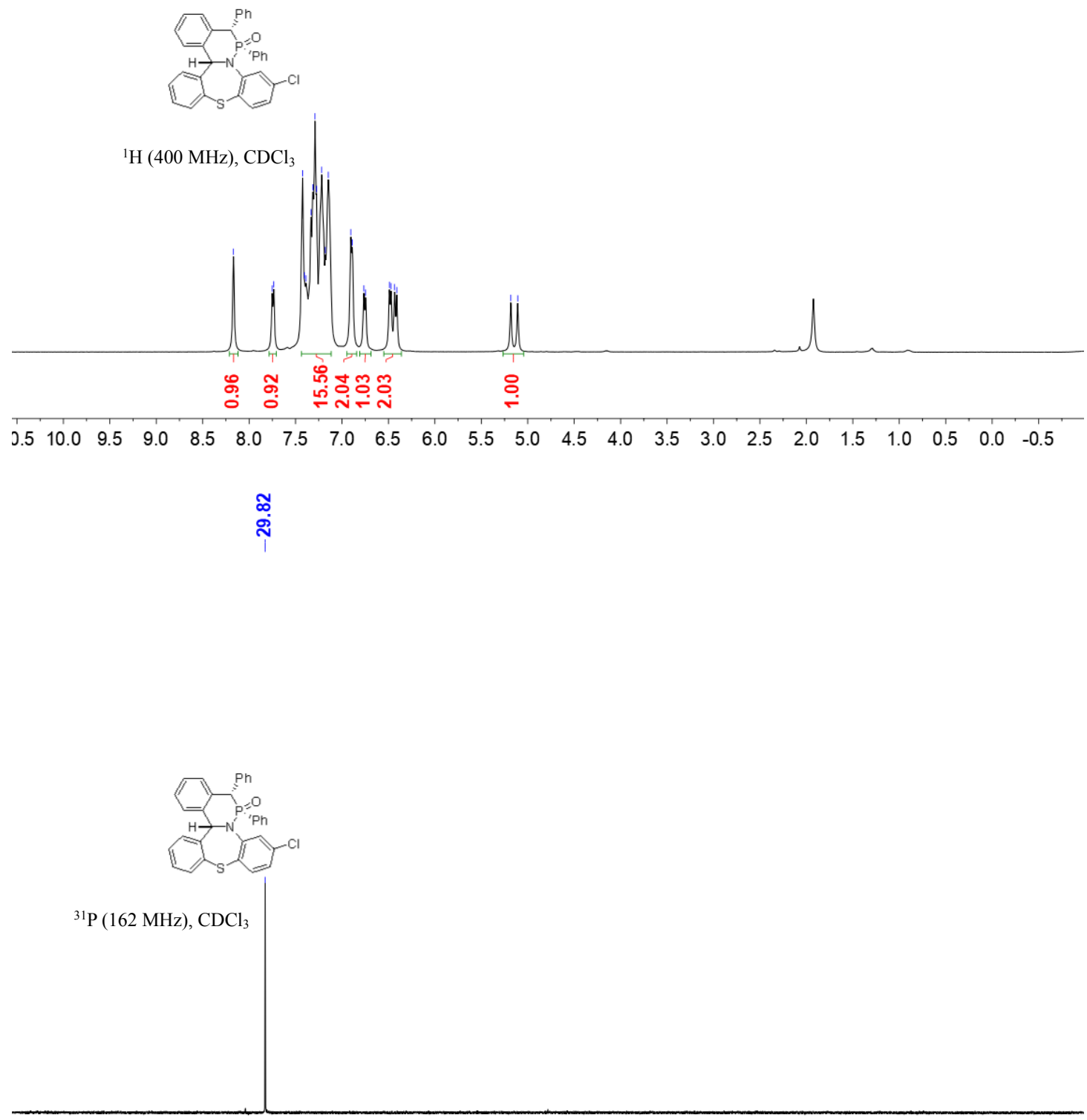

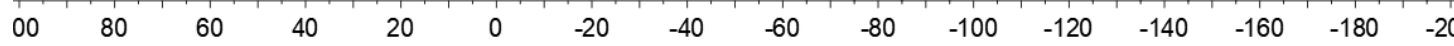




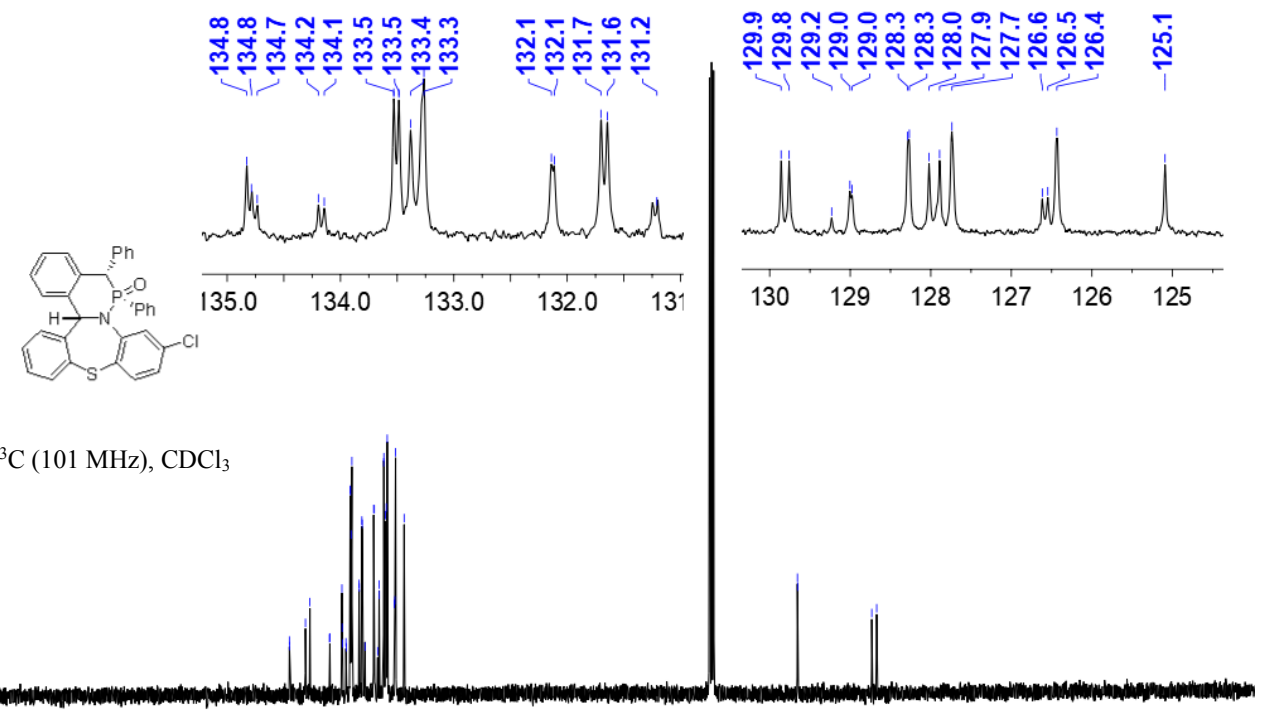

$\begin{array}{llllllllllllllllllllll}210 & 200 & 190 & 180 & 170 & 160 & 150 & 140 & 130 & 120 & 110 & 100 & 90 & 80 & 70 & 60 & 50 & 40 & 30 & 20 & 10 & 0\end{array}$

${ }^{1} \mathrm{H}(400 \mathrm{MHz}),{ }^{31} \mathrm{P}(162 \mathrm{MHz})$ and ${ }^{13} \mathrm{C}(101 \mathrm{MHz})$ spectra of compound $\mathbf{3 r}$

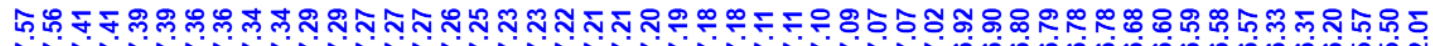

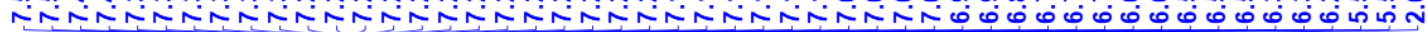

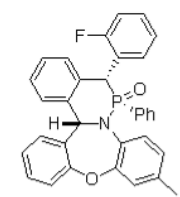

${ }^{1} \mathrm{H}(400 \mathrm{MHz}), \mathrm{CDCl}_{3}$

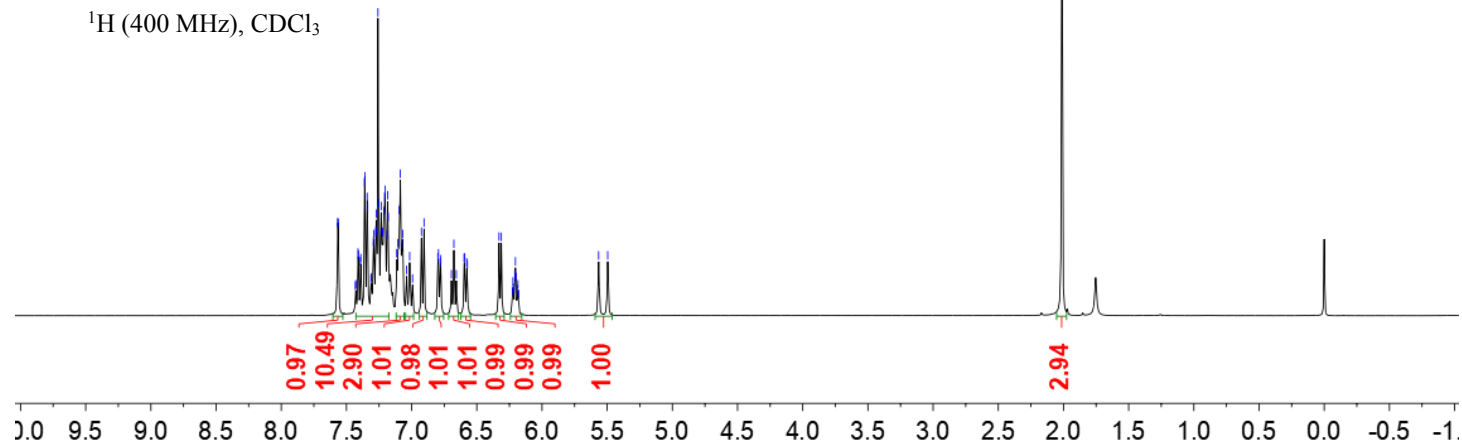


ฺฺָุ

ลิ่

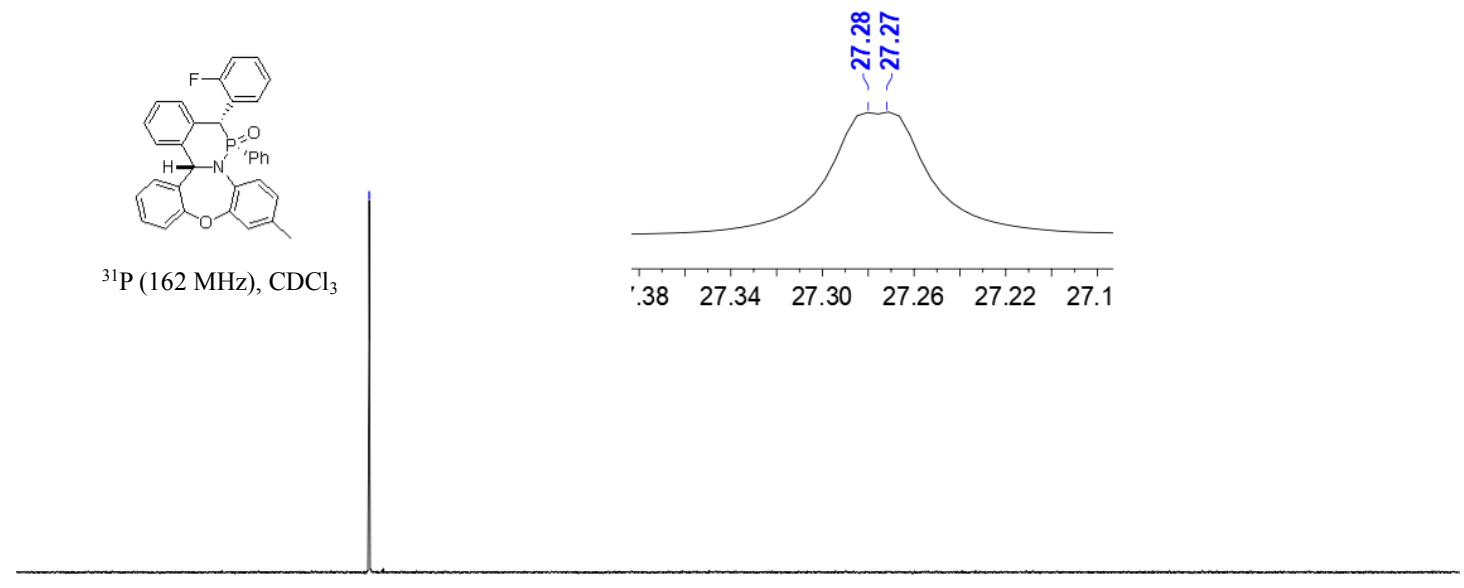

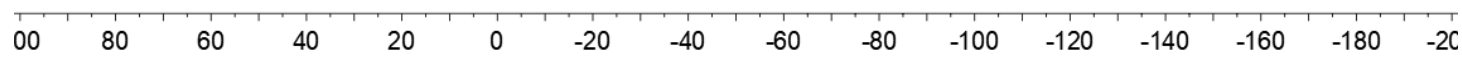

$\frac{\text { ָุ }}{\stackrel{i}{i}}$

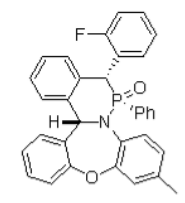

${ }^{19} \mathrm{~F}(376 \mathrm{MHz}), \mathrm{CDCl}_{3}$

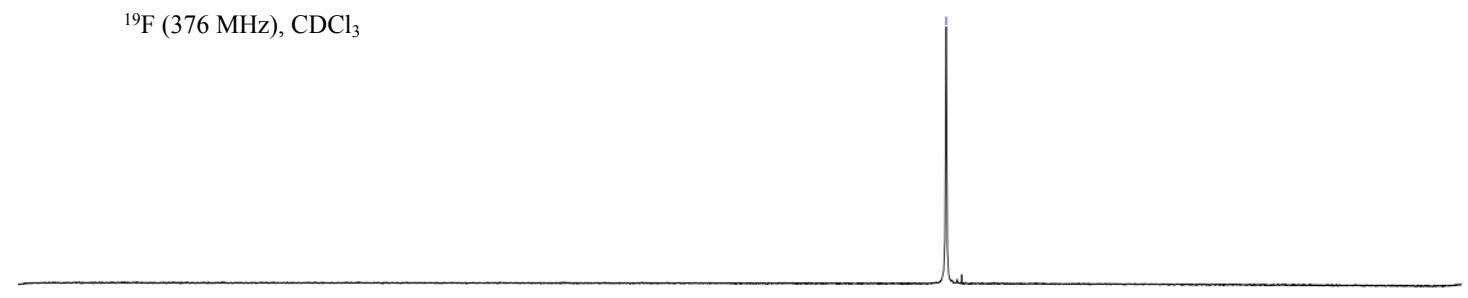

$\begin{array}{lllllllllllllllllll}-55 & -60 & -65 & -70 & -75 & -80 & -85 & -90 & -95 & -100 & -105 & -110 & -115 & -120 & -125 & -130 & -135 & -140 & -145\end{array}$ 

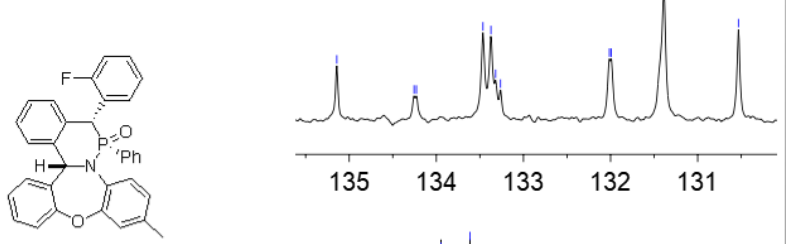

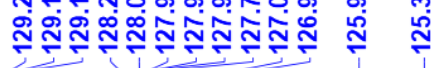

${ }^{13} \mathrm{C}(101 \mathrm{MHz}), \mathrm{CDCl}_{3}$
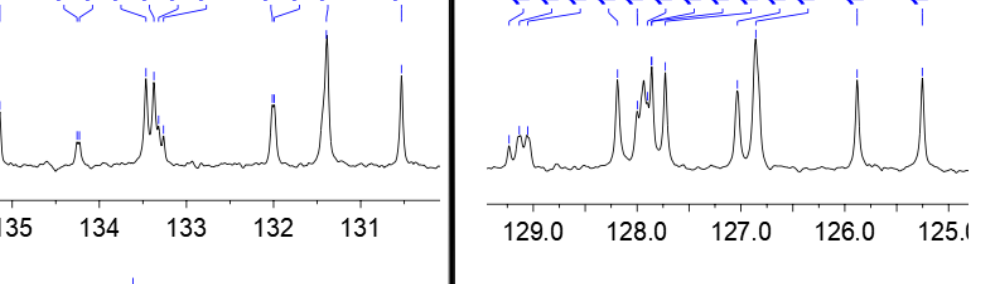

$\begin{array}{llllllllllllllllllllll}210 & 200 & 190 & 180 & 170 & 160 & 150 & 140 & 130 & 120 & 110 & 100 & 90 & 80 & 70 & 60 & 50 & 40 & 30 & 20 & 10 & 0\end{array}$

${ }^{1} \mathrm{H}(400 \mathrm{MHz}),{ }^{31} \mathrm{P}(162 \mathrm{MHz}),{ }^{19} \mathrm{~F}$ NMR $(376 \mathrm{MHz})$ and ${ }^{13} \mathrm{C}(101 \mathrm{MHz})$ spectra of compound $3 \mathbf{s}$

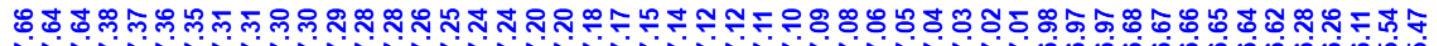

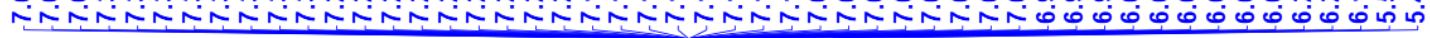

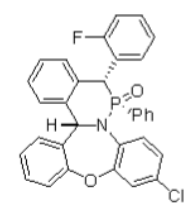

${ }^{1} \mathrm{H}(400 \mathrm{MHz}), \mathrm{CDCl}_{3}$

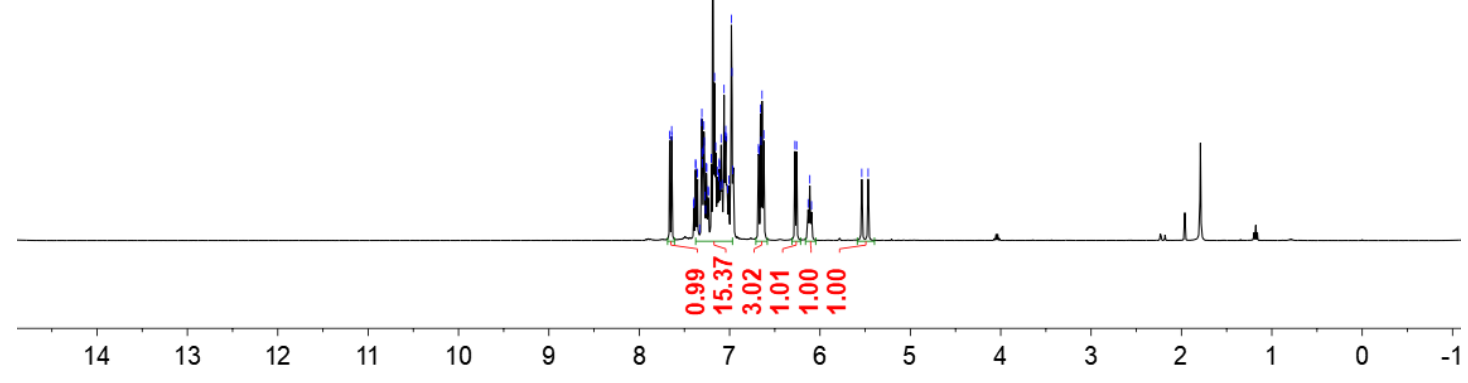




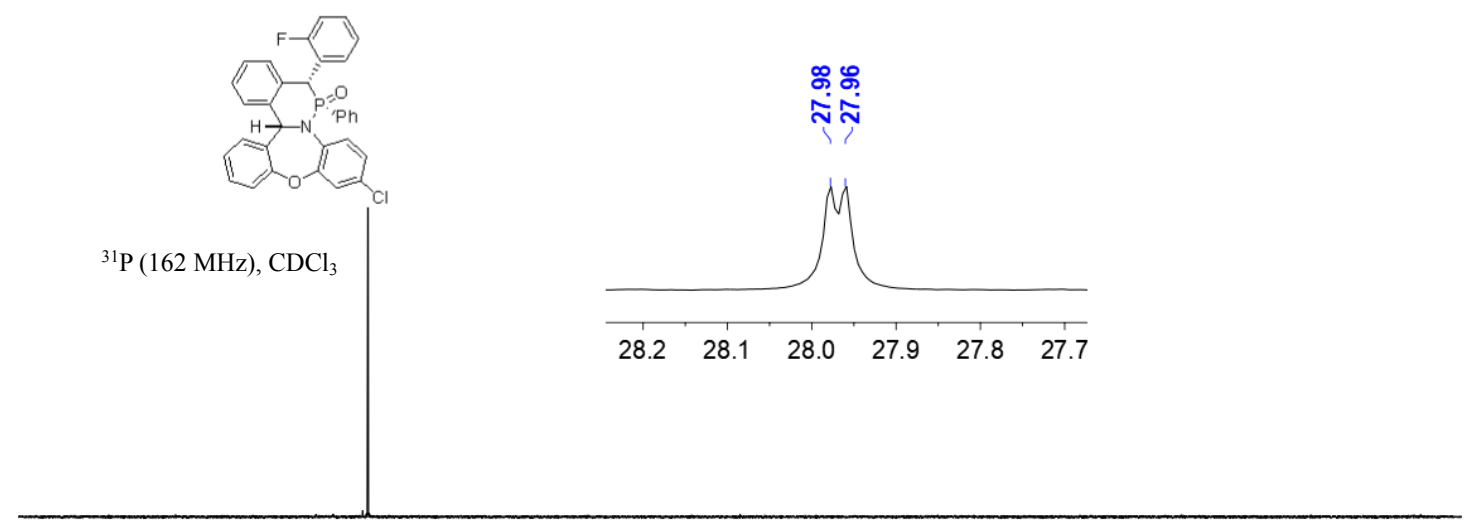

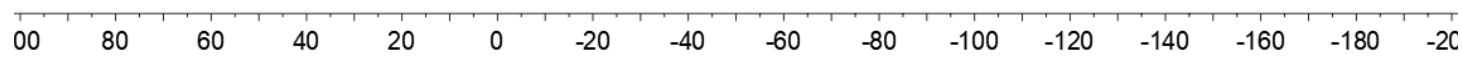

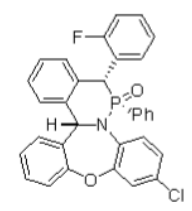

${ }^{19} \mathrm{~F}(376 \mathrm{MHz}), \mathrm{CDCl}_{3}$

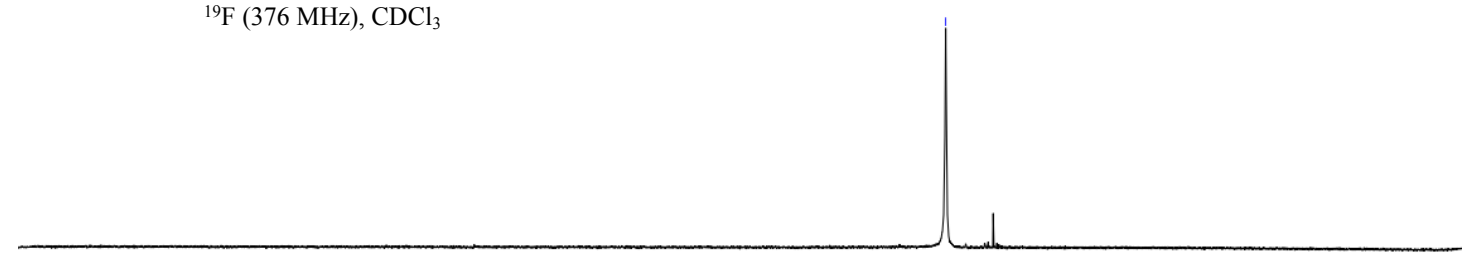

$\begin{array}{lllllllllllllllllll}-55 & -60 & -65 & -70 & -75 & -80 & -85 & -90 & -95 & -100 & -105 & -110 & -115 & -120 & -125 & -130 & -135 & -140 & -145\end{array}$ 


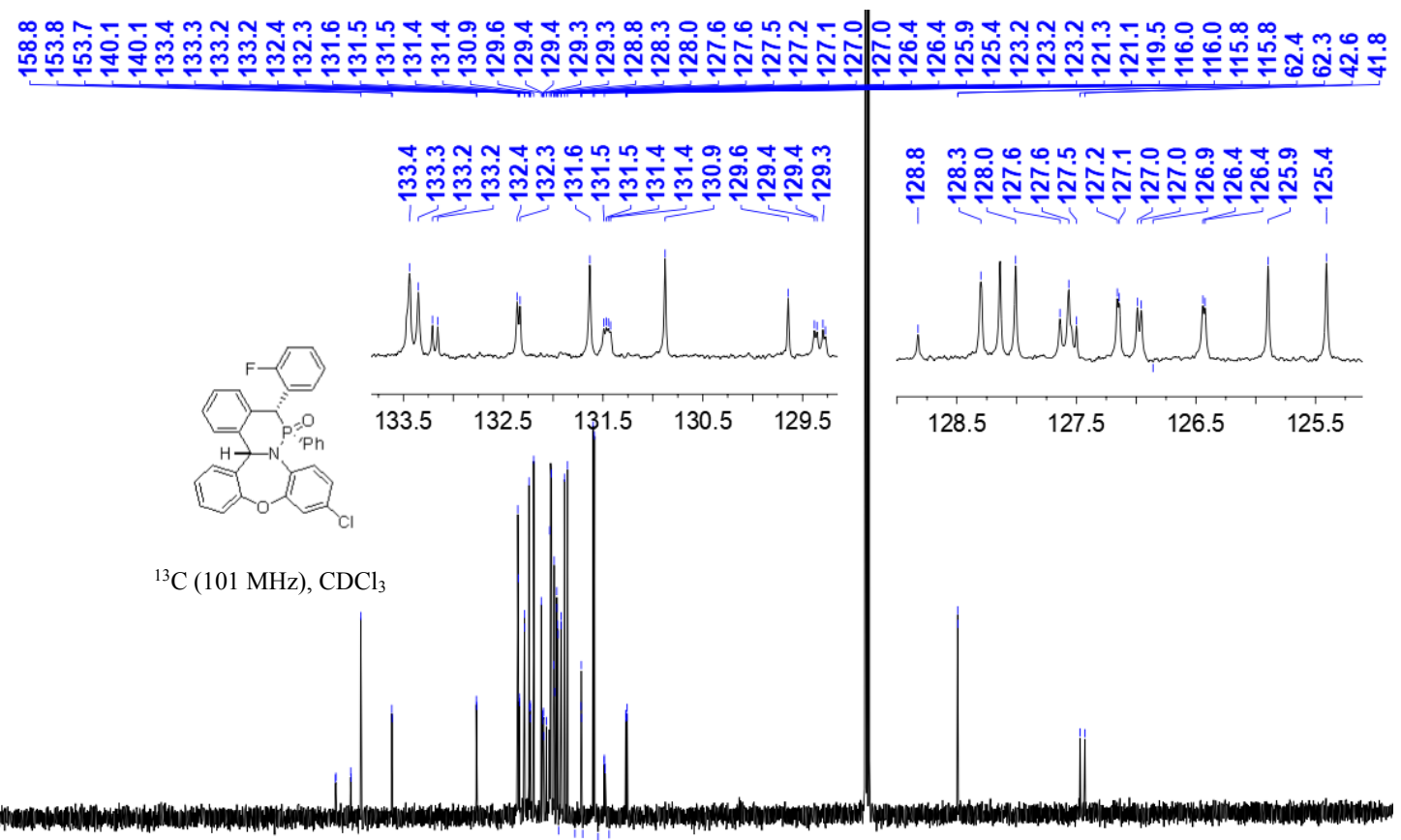

$\begin{array}{llllllllllllllllllllll}210 & 200 & 190 & 180 & 170 & 160 & 150 & 140 & 130 & 120 & 110 & 100 & 90 & 80 & 70 & 60 & 50 & 40 & 30 & 20 & 10 & 0\end{array}$

${ }^{1} \mathrm{H}(400 \mathrm{MHz}),{ }^{31} \mathrm{P}(162 \mathrm{MHz}),{ }^{19} \mathrm{~F}$ NMR $(376 \mathrm{MHz})$ and ${ }^{13} \mathrm{C}(101 \mathrm{MHz})$ spectra of compound $\mathbf{3 t}$

นํํำ

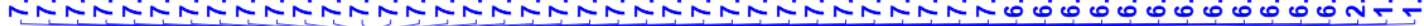
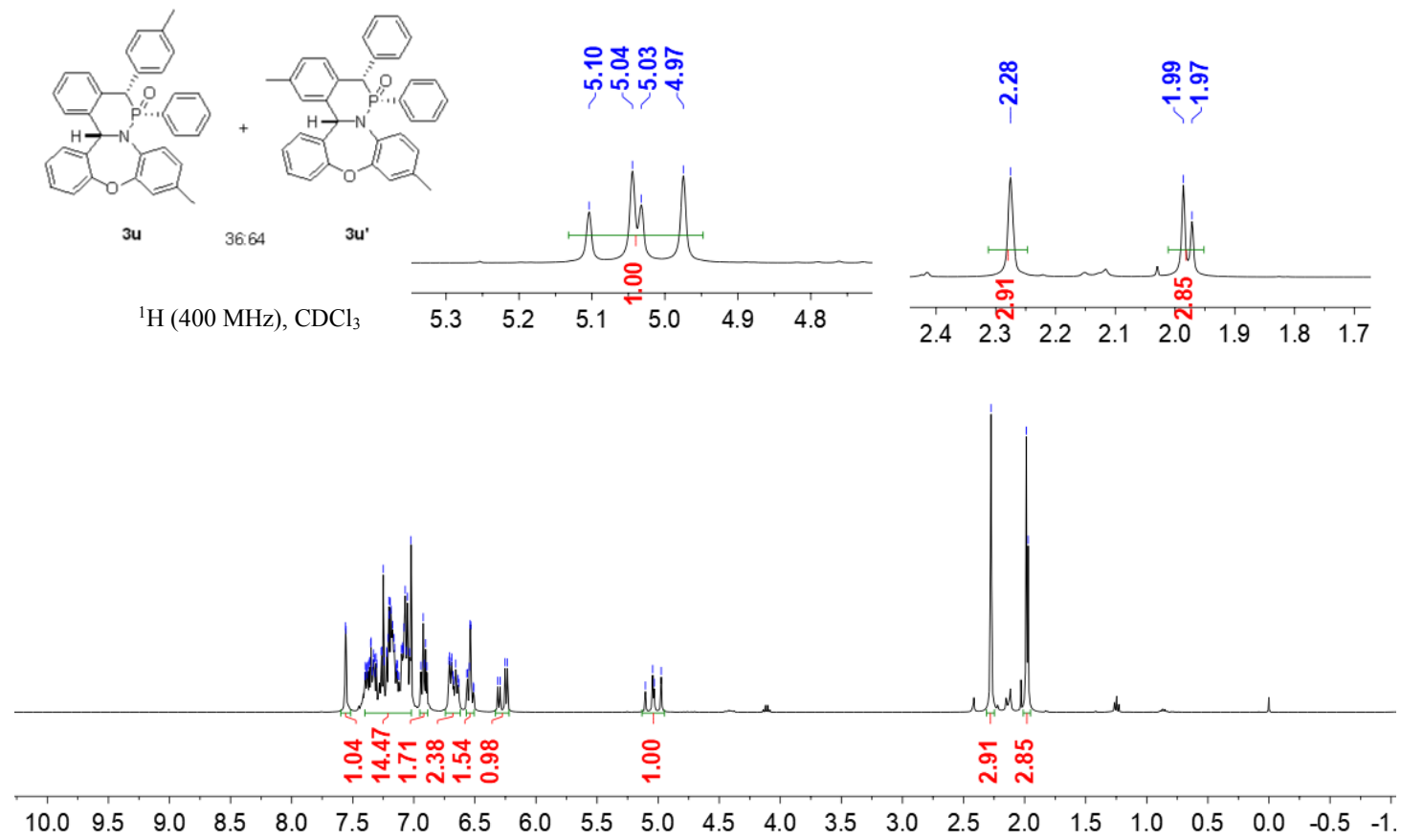


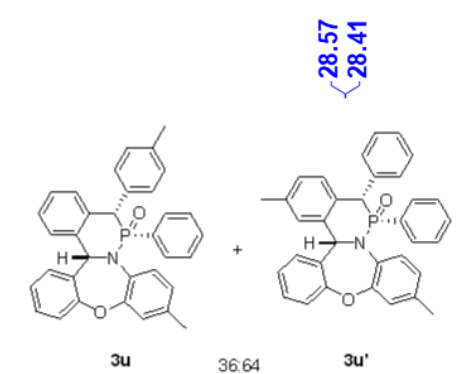

${ }^{31} \mathrm{P}(162 \mathrm{MHz}), \mathrm{CDCl}_{3}$

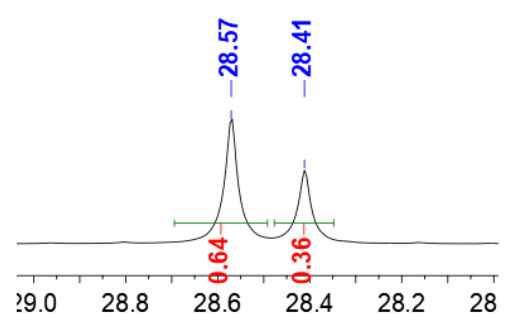

\begin{tabular}{lllllllllllllllll}
\hline & 80 & 60 & 40 & 20 & 0 & -20 & -40 & -60 & -80 & -100 & -120 & -140 & -160 & -180 & $-2 C$
\end{tabular}

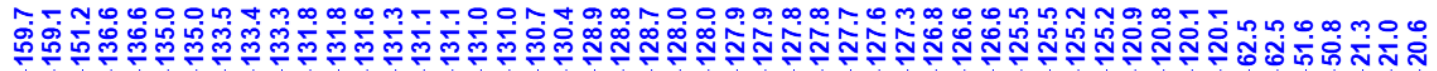

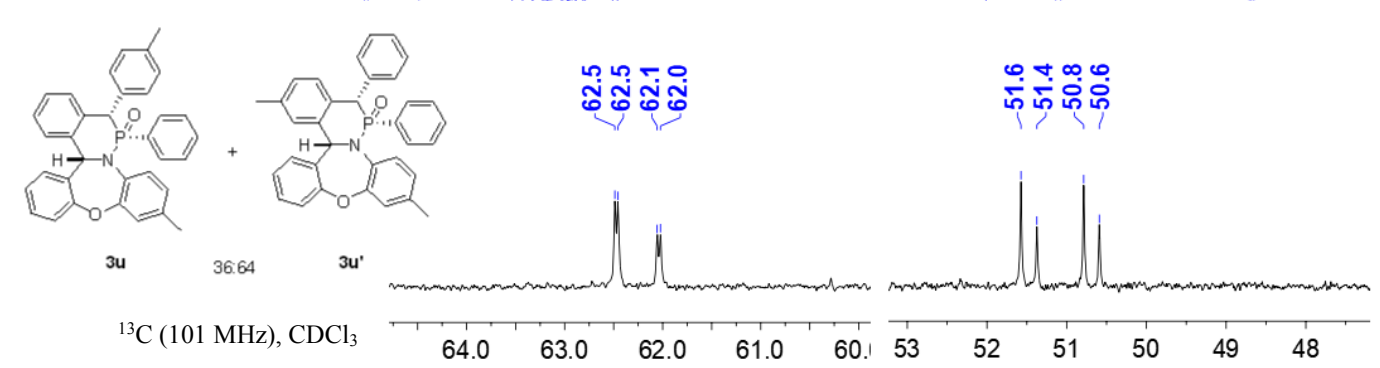

$\begin{array}{lllllllllllllllllllllll}210 & 200 & 190 & 180 & 170 & 160 & 150 & 140 & 130 & 120 & 110 & 100 & 90 & 80 & 70 & 60 & 50 & 40 & 30 & 20 & 10 & 0\end{array}$

${ }^{1} \mathrm{H}(400 \mathrm{MHz}),{ }^{31} \mathrm{P}(162 \mathrm{MHz})$ and ${ }^{13} \mathrm{C}(101 \mathrm{MHz})$ spectra of compounds $\mathbf{3 u}$ and $\mathbf{3} \mathbf{u}$ ' 


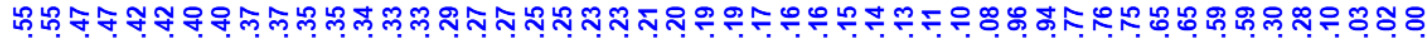

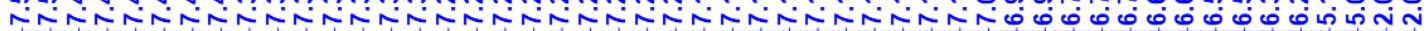

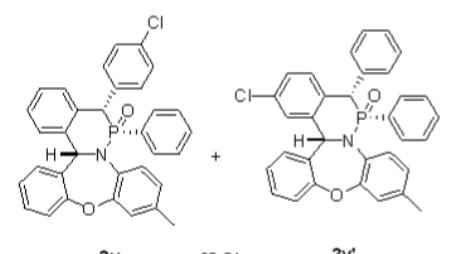

3v $69: 31 \quad 3 v^{\prime}$

${ }^{1} \mathrm{H}(400 \mathrm{MHz}), \mathrm{CDCl}_{3}$
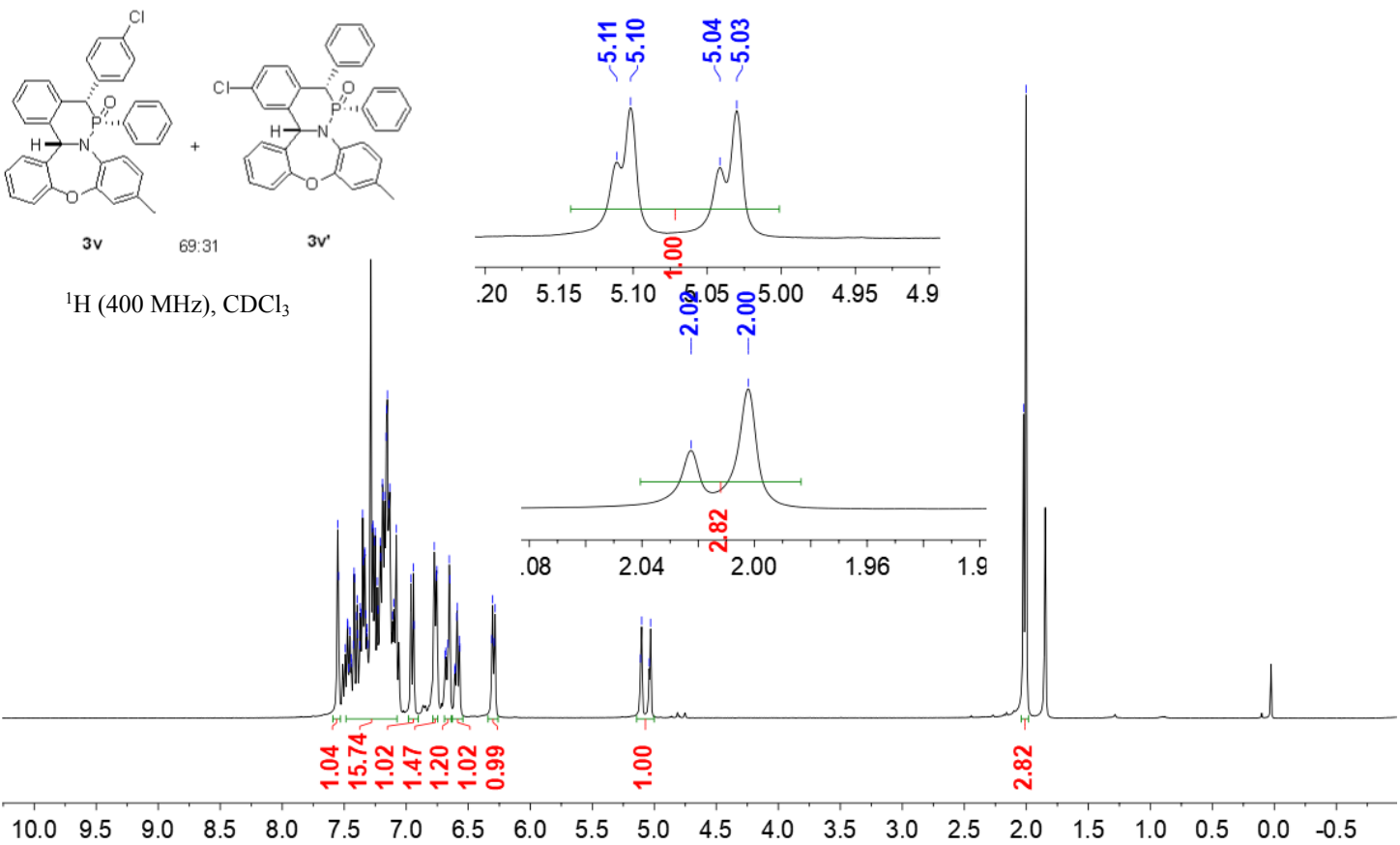

冬

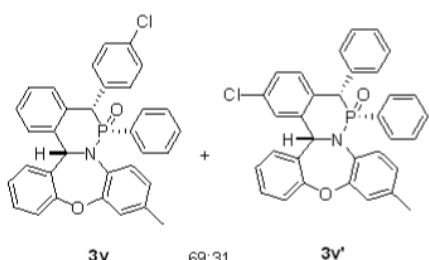

${ }^{31} \mathrm{P}(162 \mathrm{MHz}), \mathrm{CDCl}_{3}$

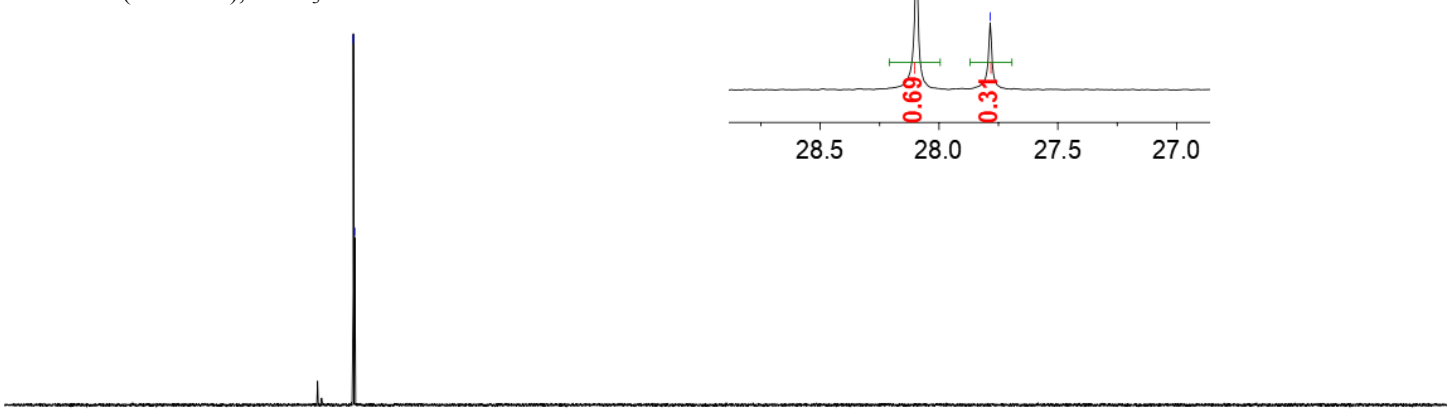

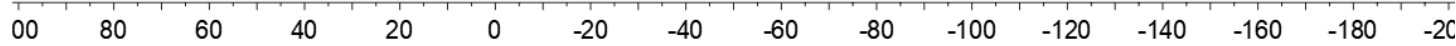




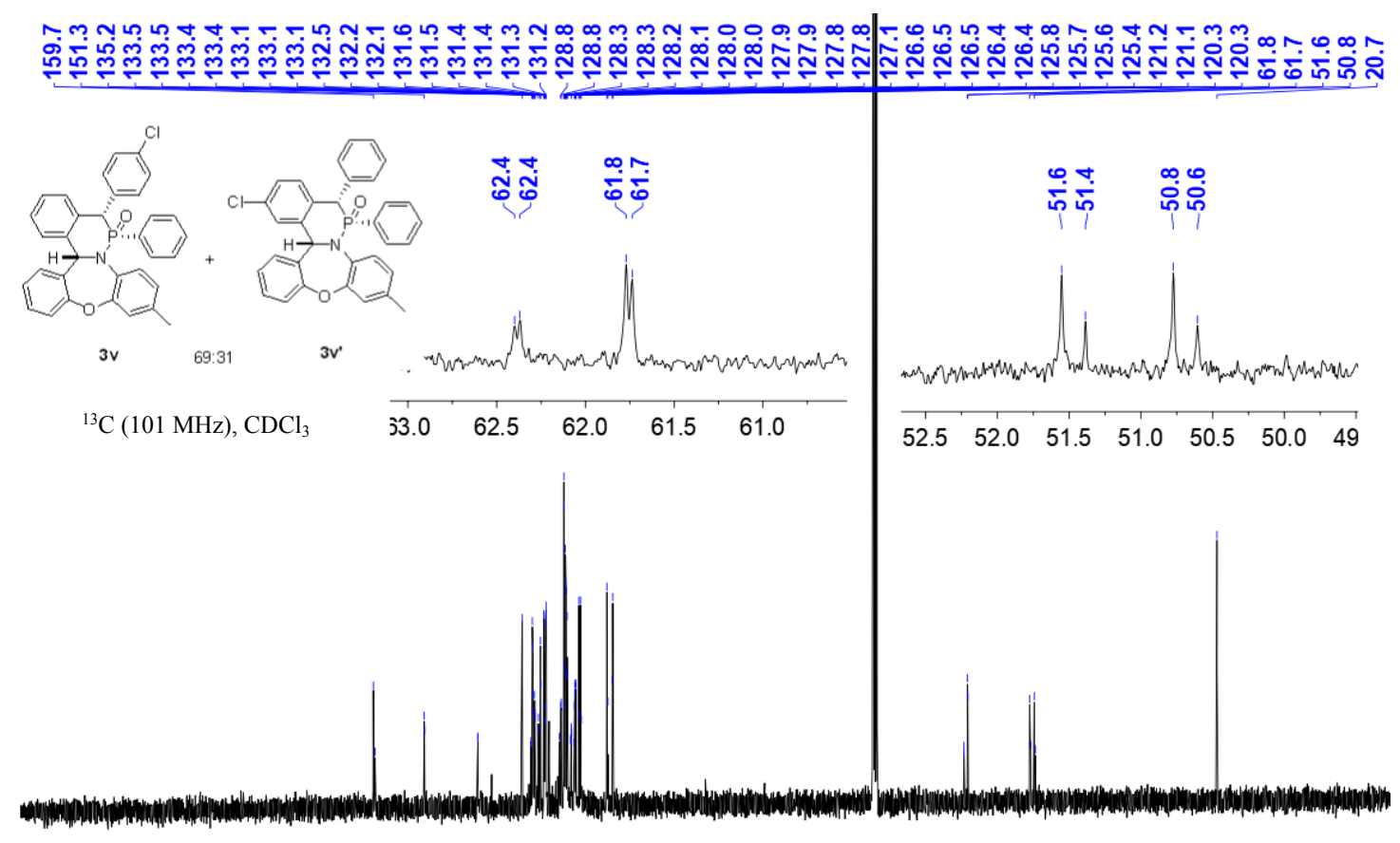

$\begin{array}{llllllllllllllllllllll}210 & 200 & 190 & 180 & 170 & 160 & 150 & 140 & 130 & 120 & 110 & 100 & 90 & 80 & 70 & 60 & 50 & 40 & 30 & 20 & 10 & 0\end{array}$

${ }^{1} \mathrm{H}(400 \mathrm{MHz}),{ }^{31} \mathrm{P}(162 \mathrm{MHz})$ and ${ }^{13} \mathrm{C}(101 \mathrm{MHz})$ spectra of compounds $\mathbf{3 v}$ and $\mathbf{3 v}$,

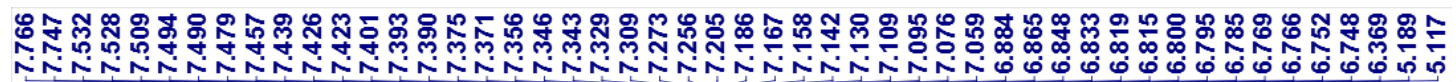

${ }^{1} \mathrm{H}(400 \mathrm{MHz}), \mathrm{CDCl}_{3}$

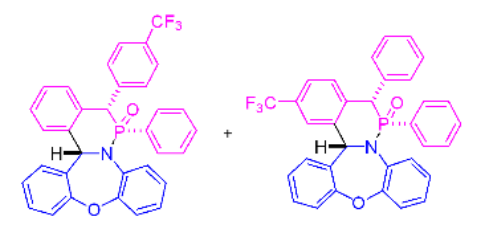

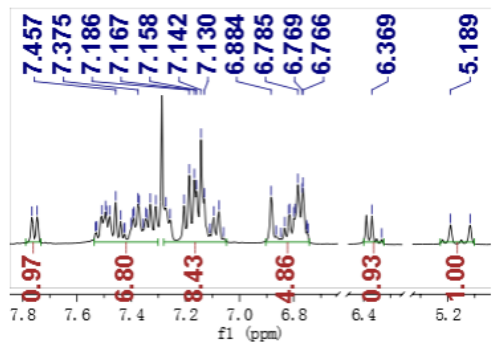

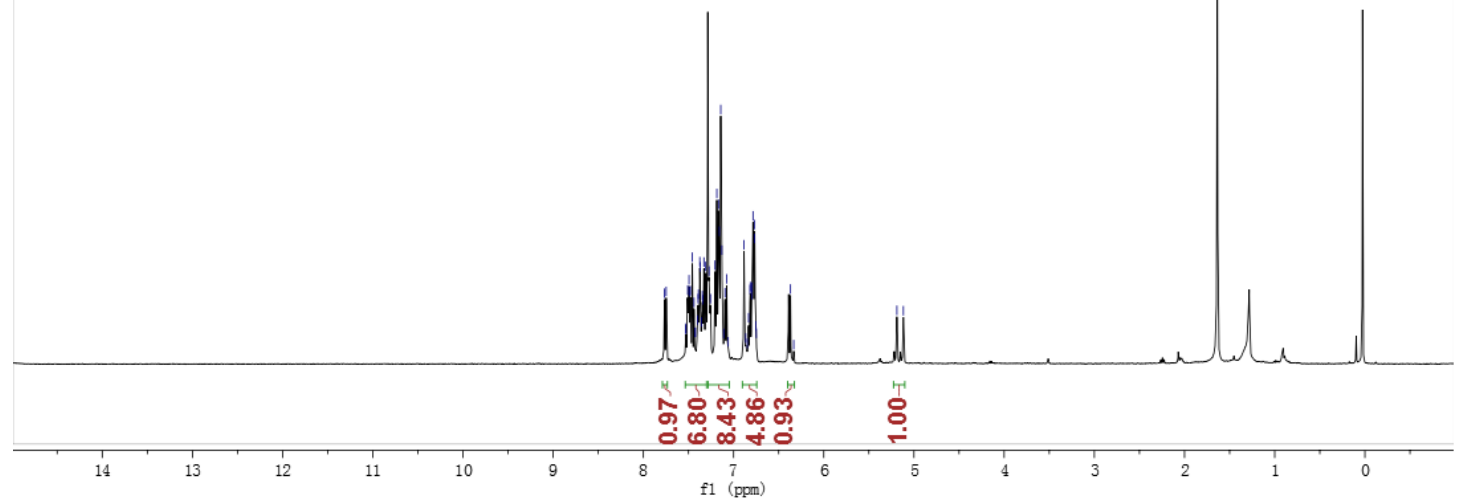




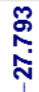

${ }^{31} \mathrm{P}(162 \mathrm{MHz}), \mathrm{CDCl}_{3}$

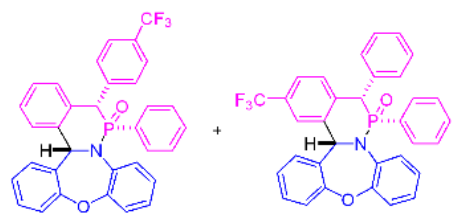

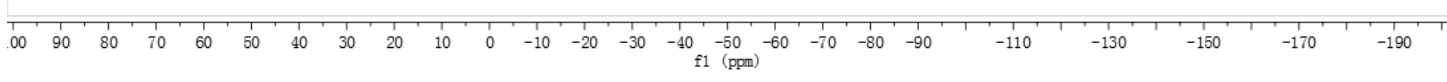

${ }^{19} \mathrm{~F}(376 \mathrm{MHz}), \mathrm{CDCl}_{3}$

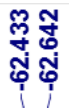
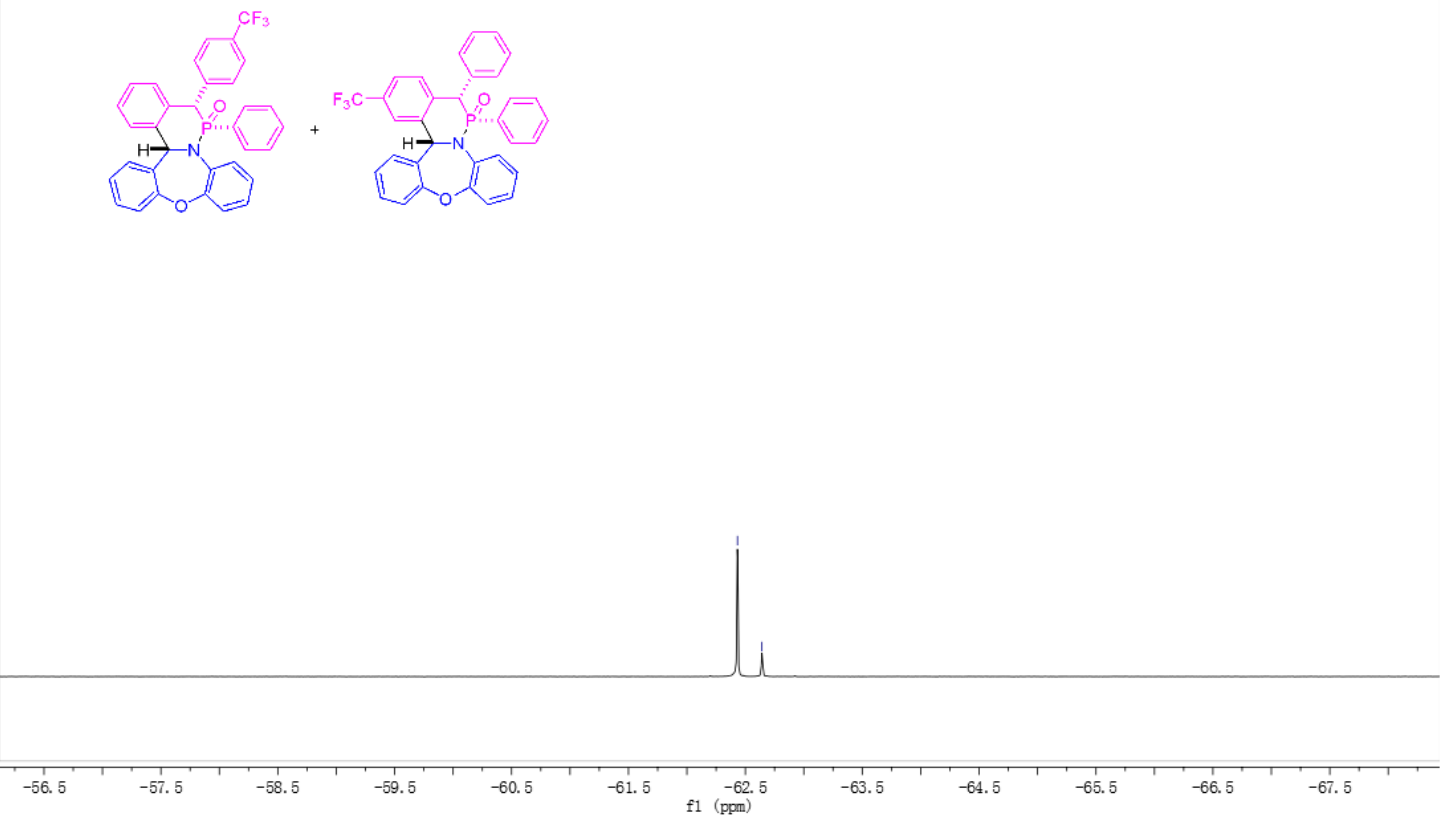

S98 

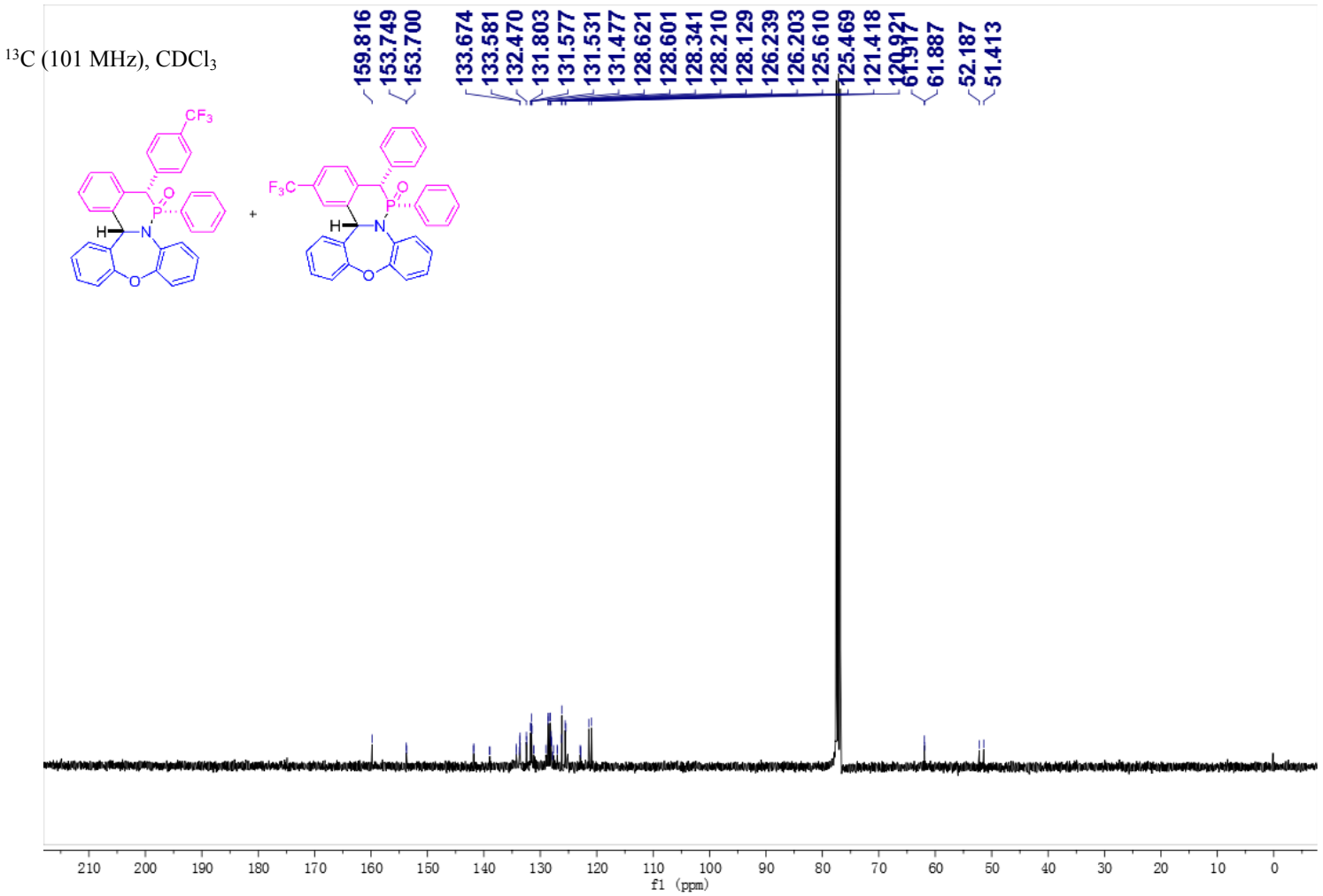

${ }^{1} \mathrm{H}(400 \mathrm{MHz}),{ }^{31} \mathrm{P}(162 \mathrm{MHz}),{ }^{19} \mathrm{~F}(376 \mathrm{MHz})$, and ${ }^{13} \mathrm{C}(101 \mathrm{MHz})$ spectra of compound $\mathbf{3 w}$ and $3 \mathbf{w}^{\prime}$
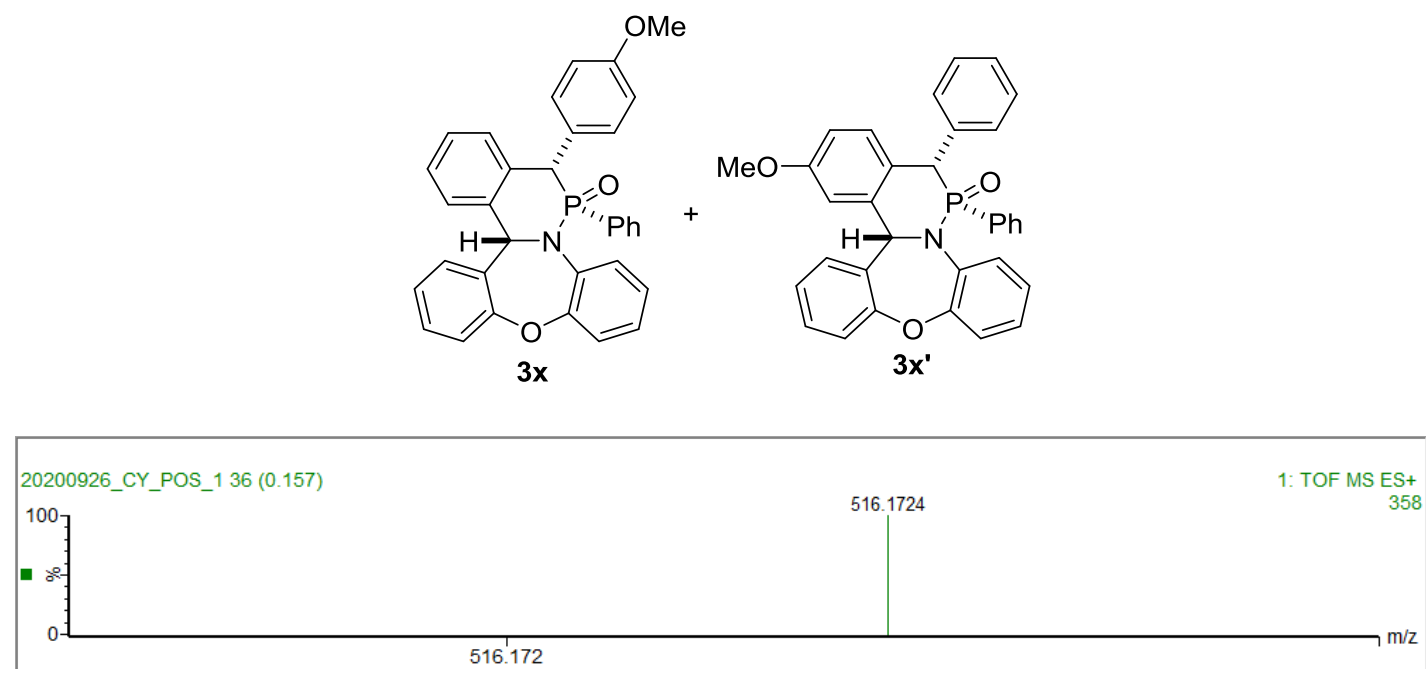

HRMS (ESI) calcd for $\mathrm{C}_{33} \mathrm{H}_{27} \mathrm{NO}_{3} \mathrm{P}^{+}[\mathrm{M}+\mathrm{H}]^{+} m / z$ : 516.1723; found 516.1724.

HRMS (ESI) spectrum of compound $\mathbf{3 x}$ and $\mathbf{3} \mathbf{x}$ 


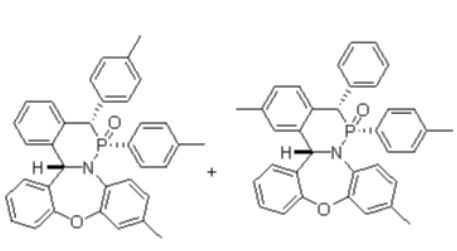

36. 64

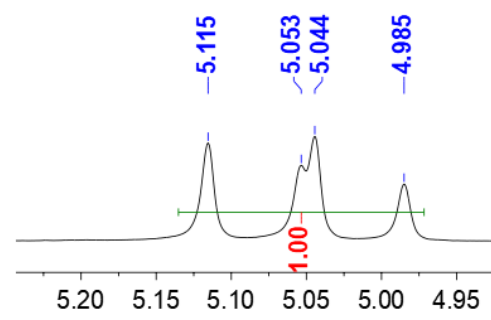

${ }^{1} \mathrm{H}(400 \mathrm{MHz}), \mathrm{CDCl}_{3}$

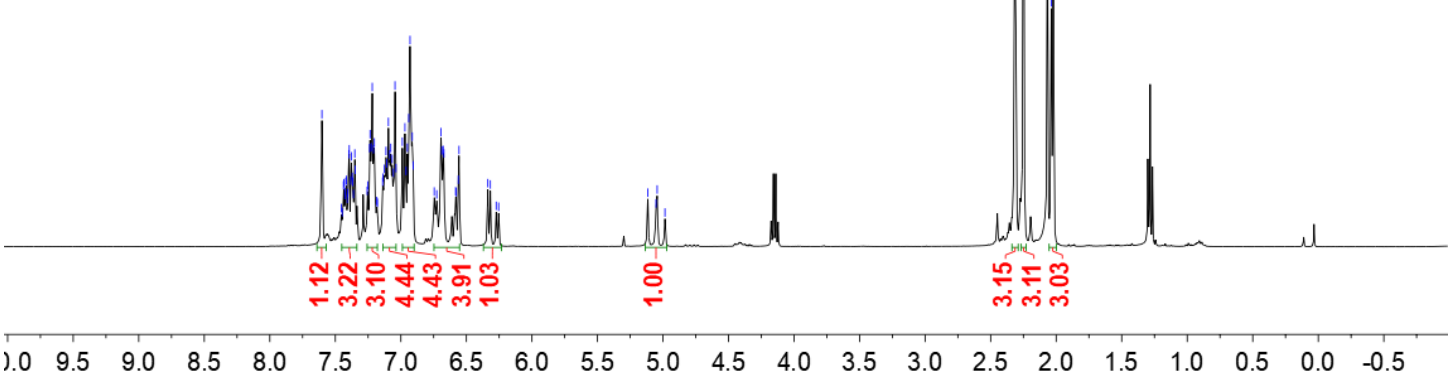

ธิธ

ชู่

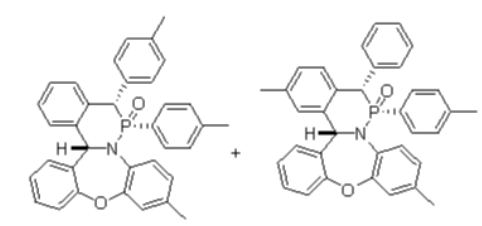

36.64

${ }^{31} \mathrm{P}(162 \mathrm{MHz}), \mathrm{CDCl}_{3}$

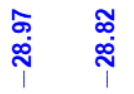

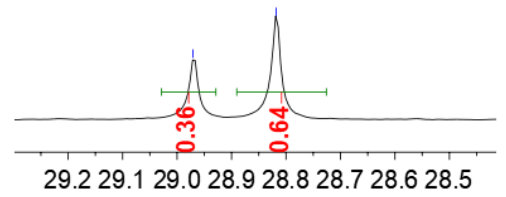

$\begin{array}{lllllllllllllllll} & 00 & 80 & 60 & 40 & 20 & 0 & -20 & -40 & -60 & -80 & -100 & -120 & -140 & -160 & -180 & -2 C\end{array}$ 


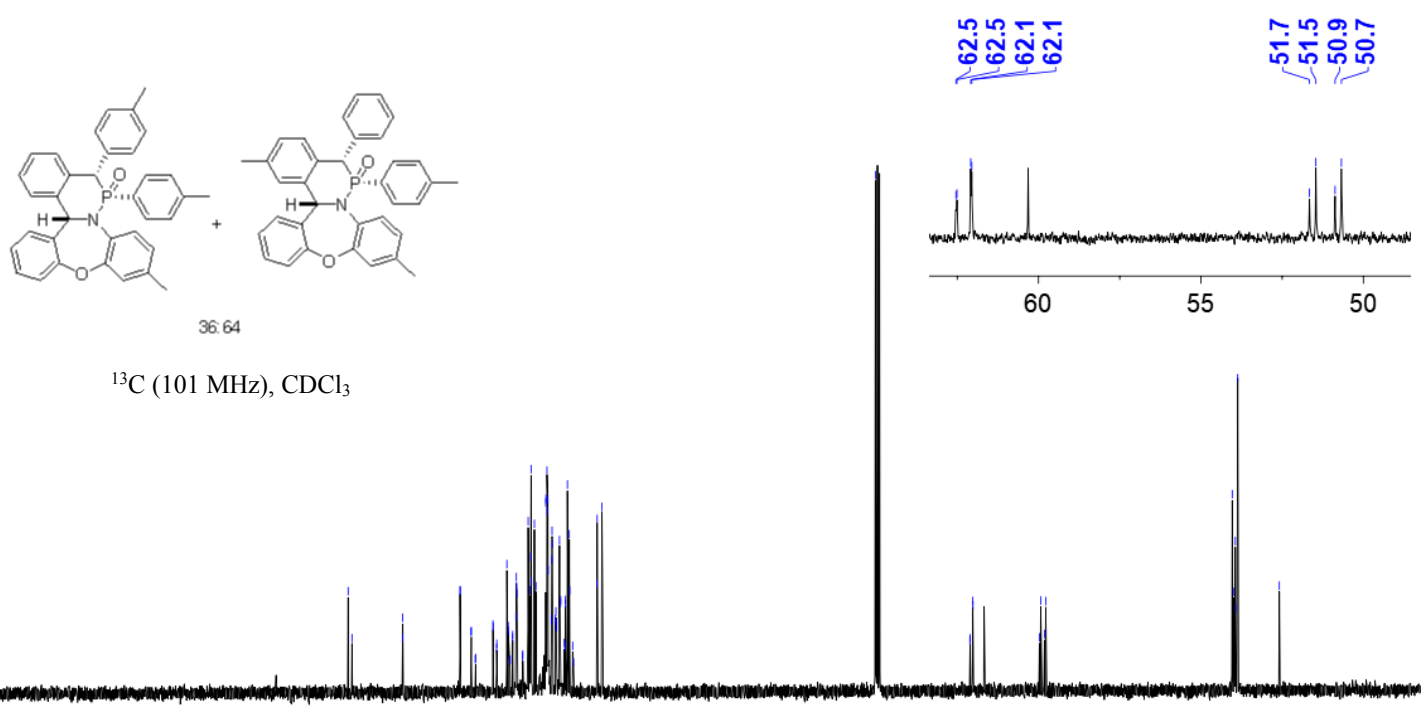

$\begin{array}{llllllllllllllllllllll}210 & 200 & 190 & 180 & 170 & 160 & 150 & 140 & 130 & 120 & 110 & 100 & 90 & 80 & 70 & 60 & 50 & 40 & 30 & 20 & 10 & 0\end{array}$

${ }^{1} \mathrm{H}(400 \mathrm{MHz}),{ }^{31} \mathrm{P}(162 \mathrm{MHz})$ and ${ }^{13} \mathrm{C}(101 \mathrm{MHz})$ spectra of compounds $\mathbf{3 y}$ and $\mathbf{3 y}$ '

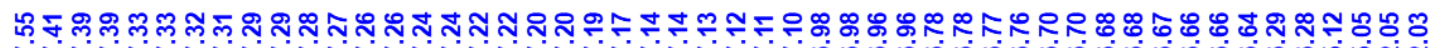

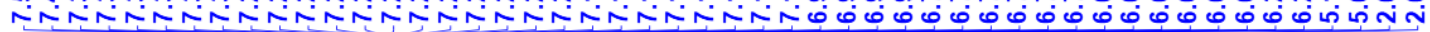

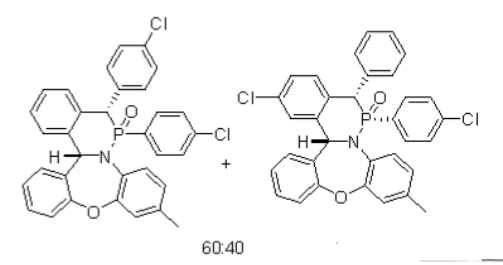

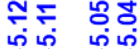

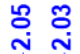

${ }^{1} \mathrm{H}(400 \mathrm{MHz}), \mathrm{CDCl}_{3}$
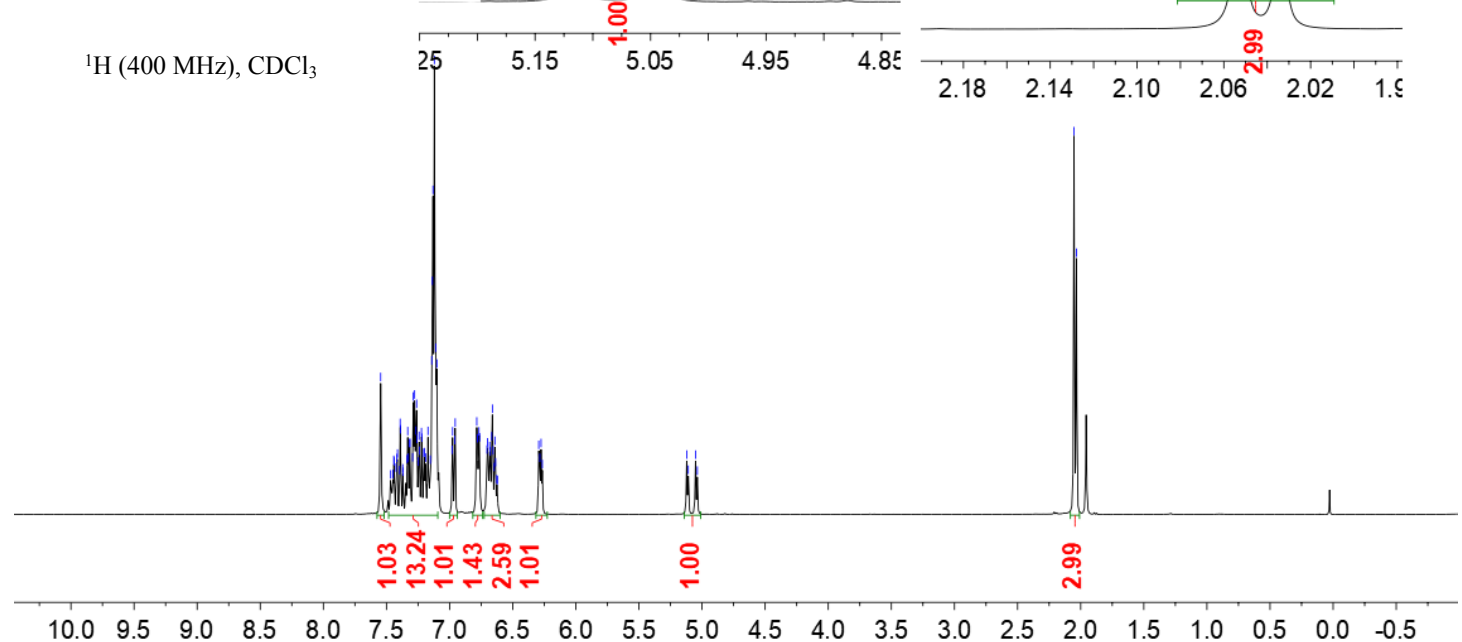


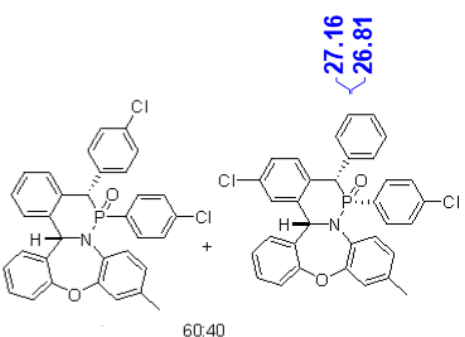

${ }^{31} \mathrm{P}(162 \mathrm{MHz}), \mathrm{CDCl}_{3}$

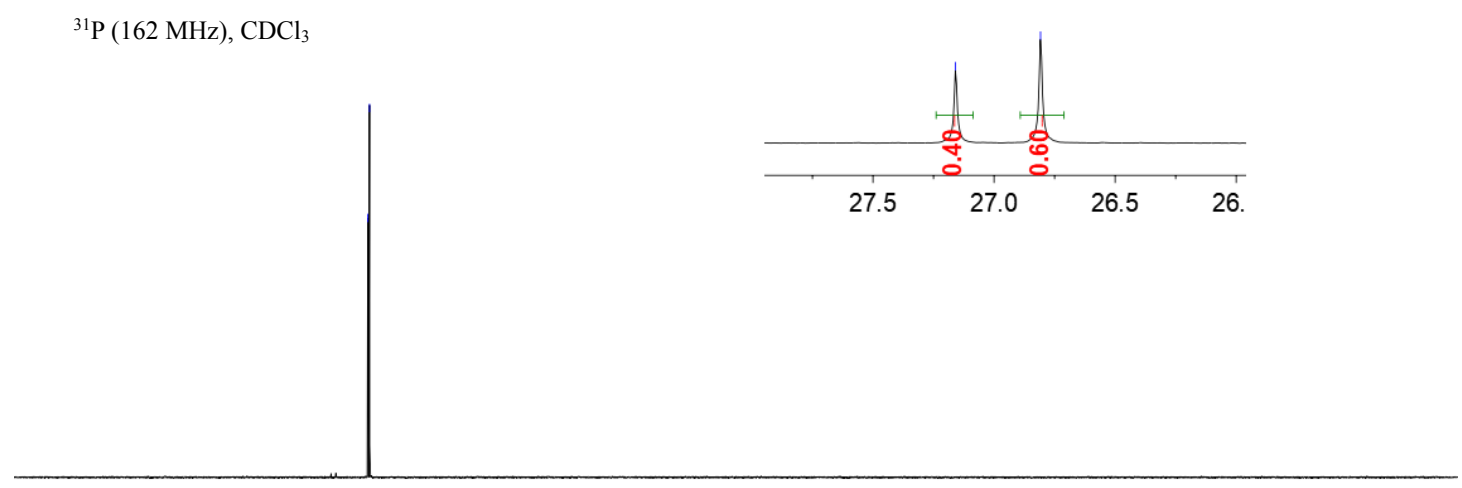

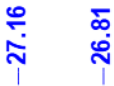

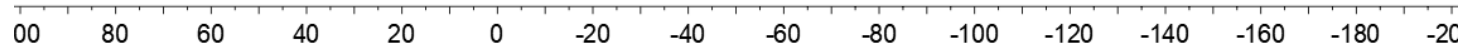

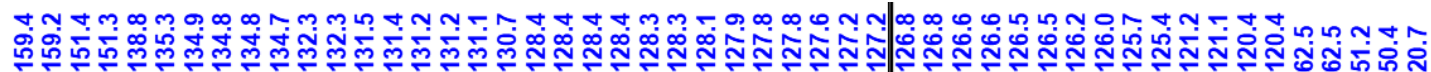

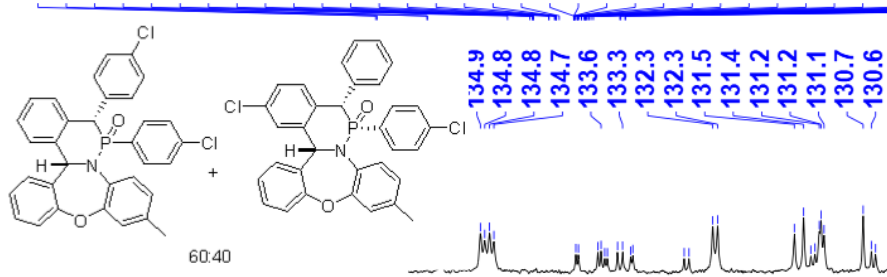

${ }^{13} \mathrm{C}(101 \mathrm{MHz}), \mathrm{CDCl}_{3}$

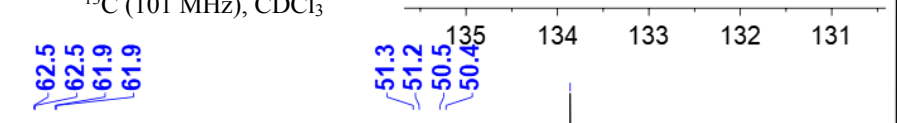

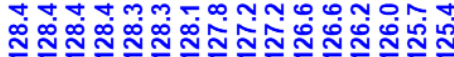

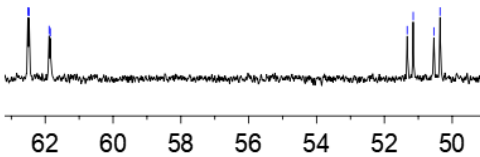

$\begin{array}{llllllllllllllllllllll}210 & 200 & 190 & 180 & 170 & 160 & 150 & 140 & 130 & 120 & 110 & 100 & 90 & 80 & 70 & 60 & 50 & 40 & 30 & 20 & 10 & 0\end{array}$

${ }^{1} \mathrm{H}(400 \mathrm{MHz}),{ }^{31} \mathrm{P}(162 \mathrm{MHz})$ and ${ }^{13} \mathrm{C}(101 \mathrm{MHz})$ spectra of compounds $\mathbf{3 z}$ and $\mathbf{3 z}{ }^{\prime}$ 

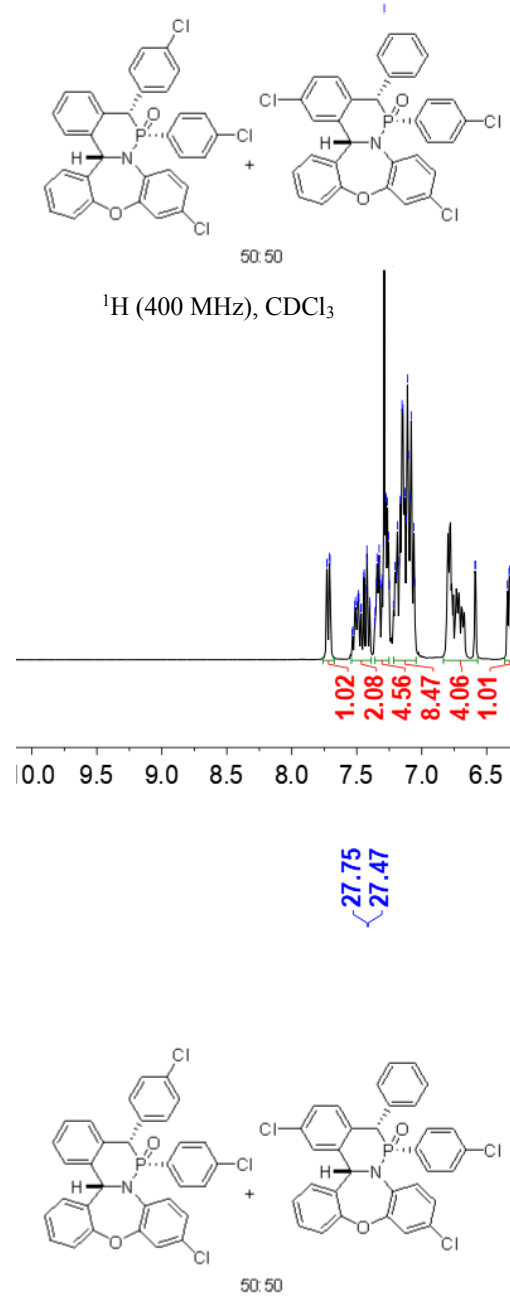

${ }^{31} \mathrm{P}(162 \mathrm{MHz}), \mathrm{CDCl}_{3}$

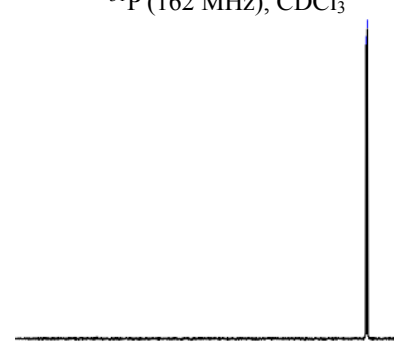

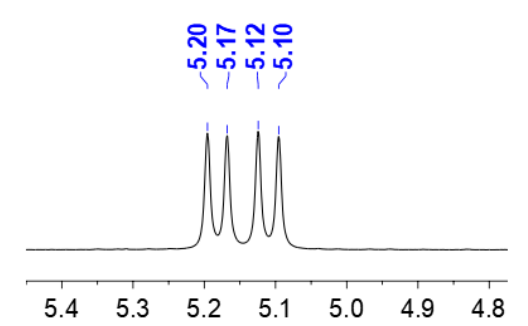

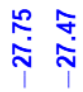

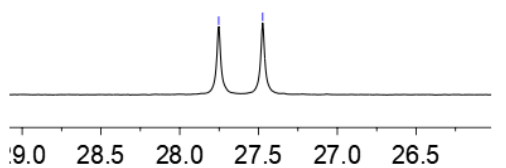

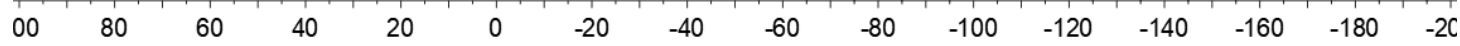




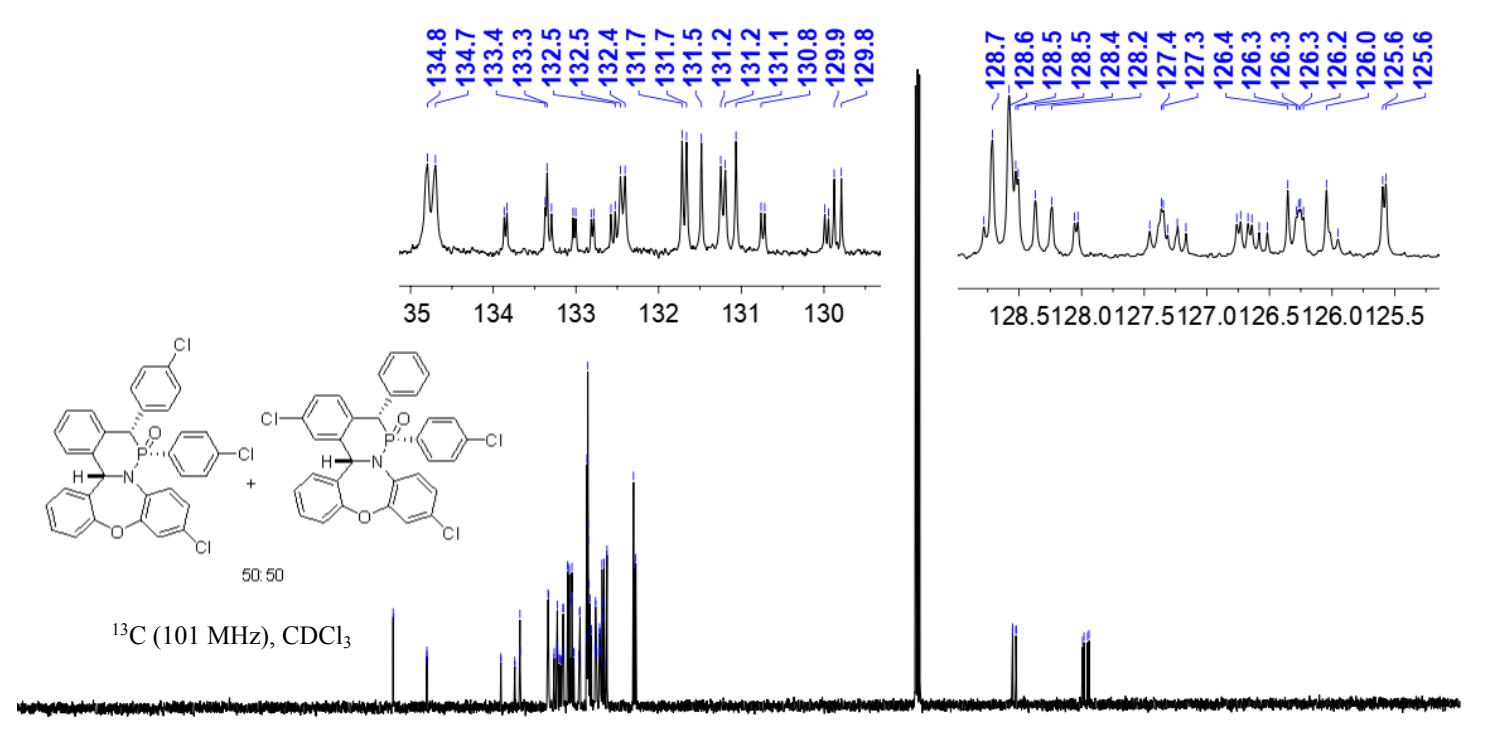

$\begin{array}{llllllllllllllllllllll}210 & 200 & 190 & 180 & 170 & 160 & 150 & 140 & 130 & 120 & 110 & 100 & 90 & 80 & 70 & 60 & 50 & 40 & 30 & 20 & 10 & 0\end{array}$

${ }^{1} \mathrm{H}(400 \mathrm{MHz}),{ }^{31} \mathrm{P}(162 \mathrm{MHz})$ and ${ }^{13} \mathrm{C}(101 \mathrm{MHz})$ spectra of compounds 3aa and 3aa'

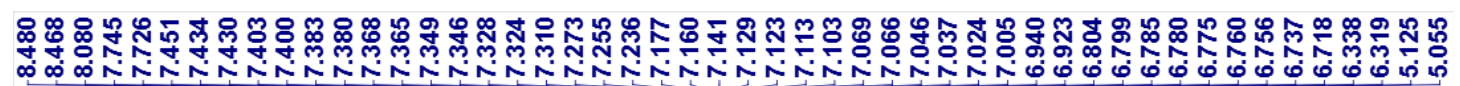

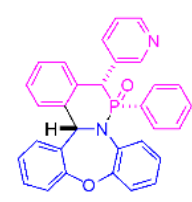

${ }^{1} \mathrm{H}(400 \mathrm{MHz}), \mathrm{CDCl}_{3}$
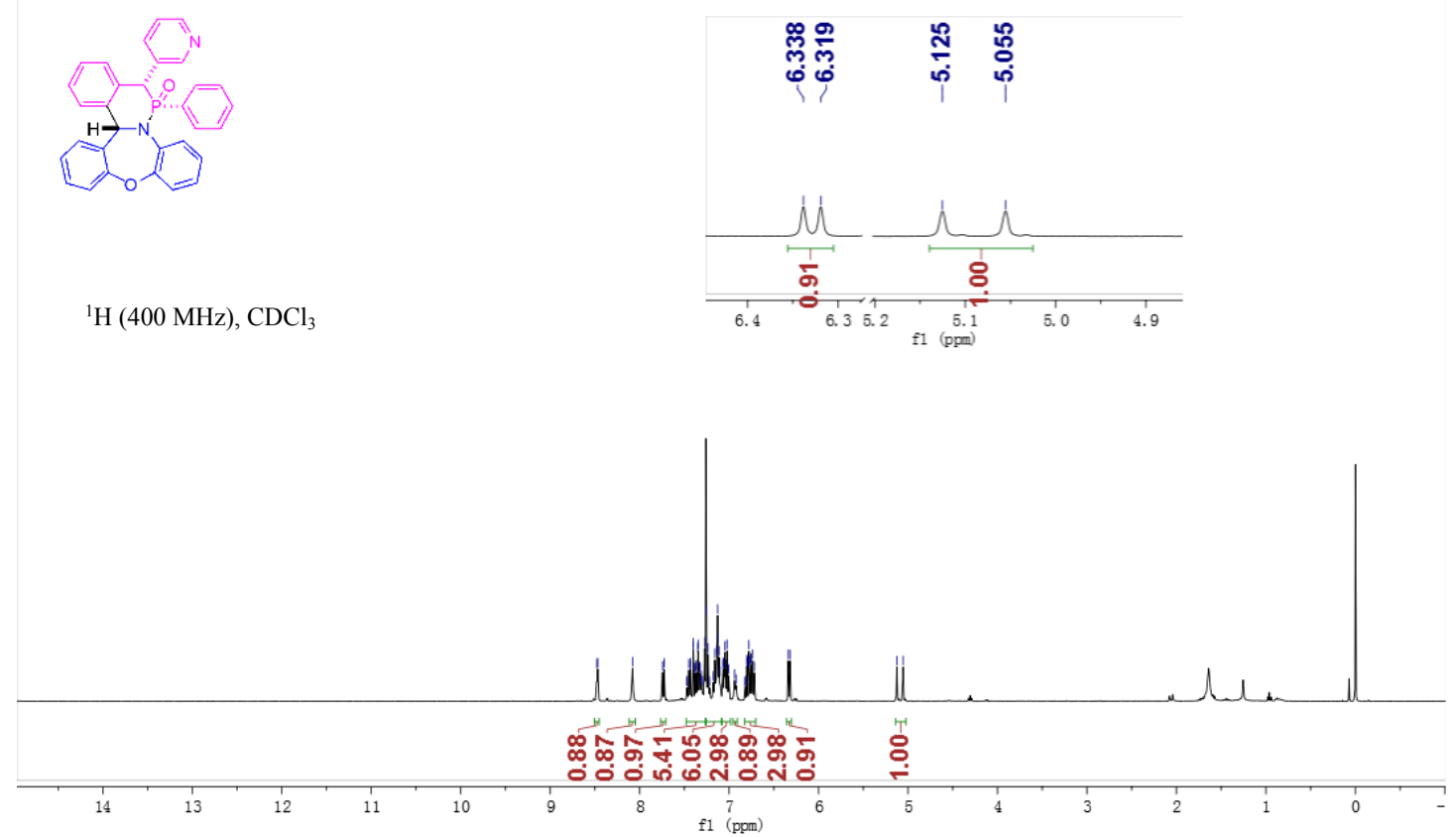


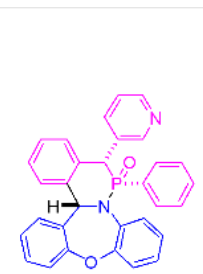

$\stackrel{\stackrel{0}{\infty}}{\stackrel{\infty}{\sim}}$

${ }^{31} \mathrm{P}(162 \mathrm{MHz}), \mathrm{CDCl}_{3}$

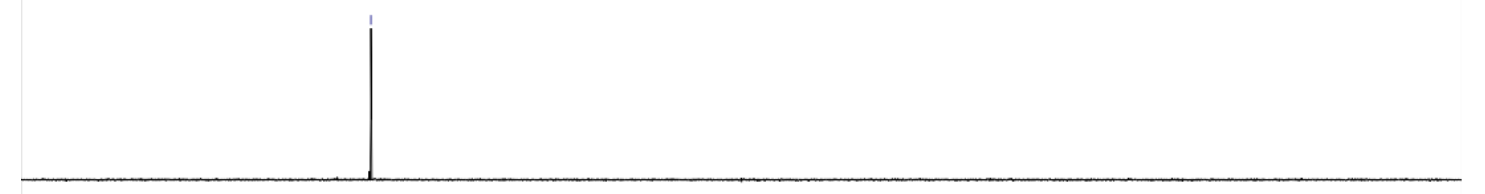

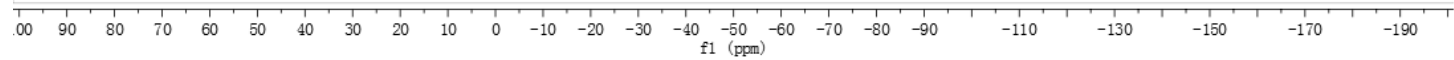
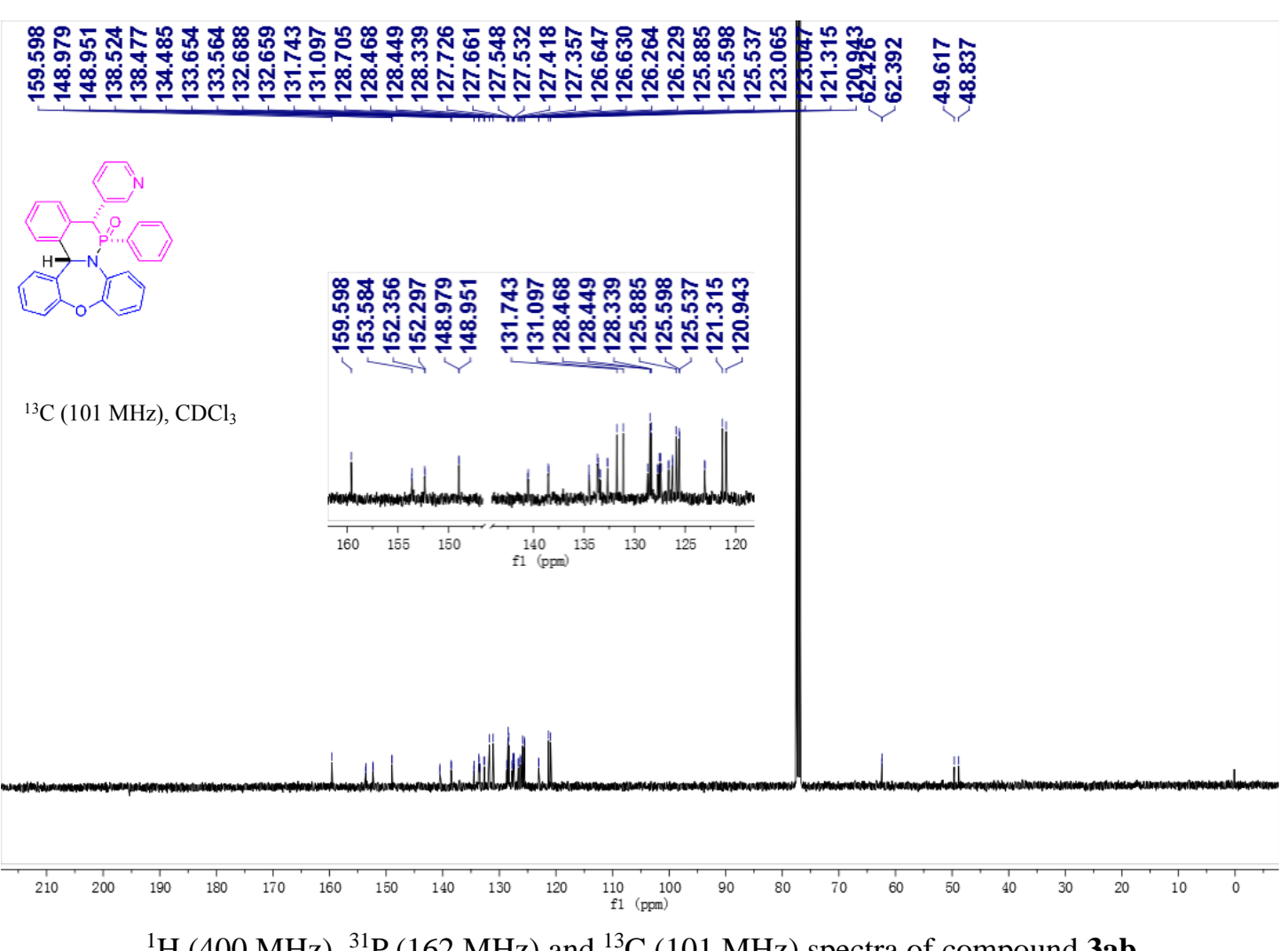

${ }^{1} \mathrm{H}(400 \mathrm{MHz}),{ }^{31} \mathrm{P}(162 \mathrm{MHz})$ and ${ }^{13} \mathrm{C}(101 \mathrm{MHz})$ spectra of compound 3ab 


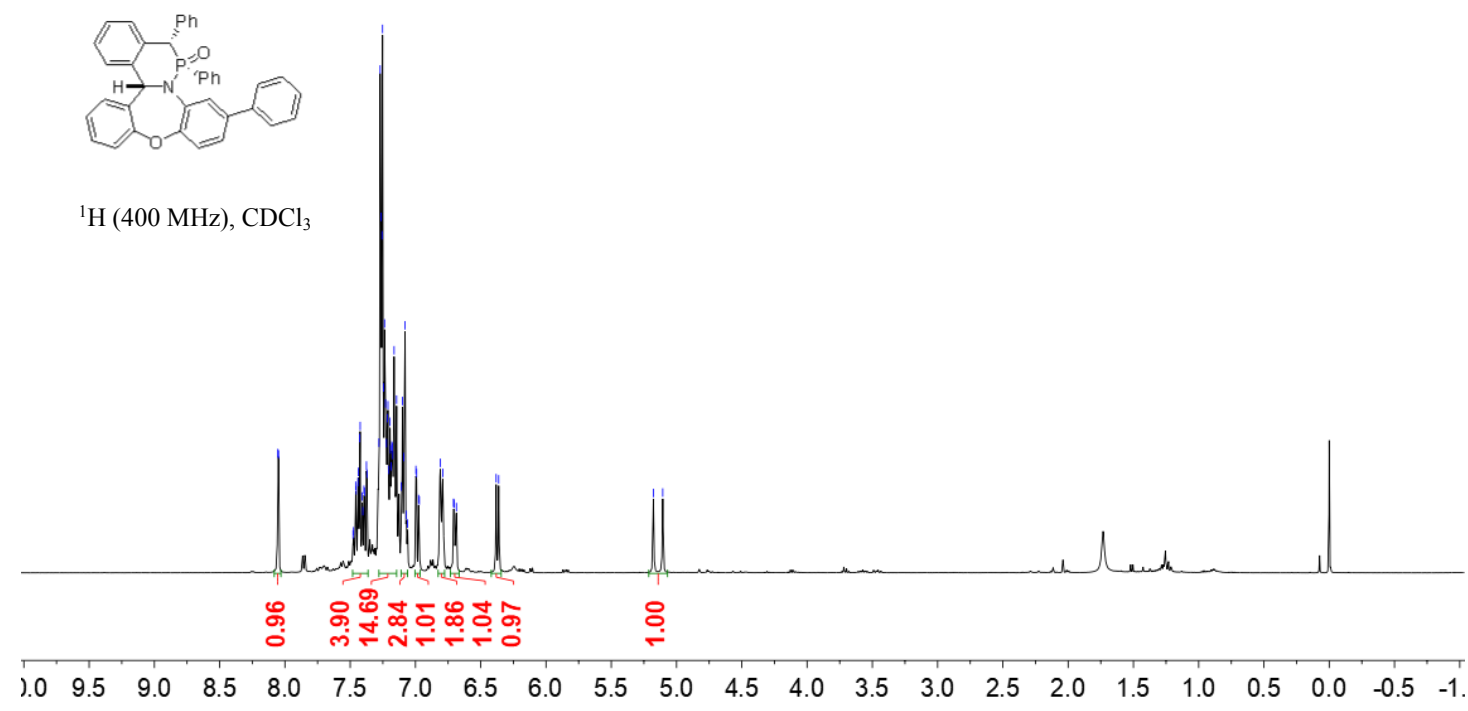

\section{\&:}

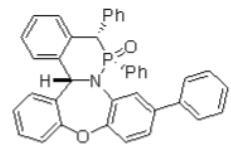

${ }^{31} \mathrm{P}(162 \mathrm{MHz}), \mathrm{CDCl}_{3}$

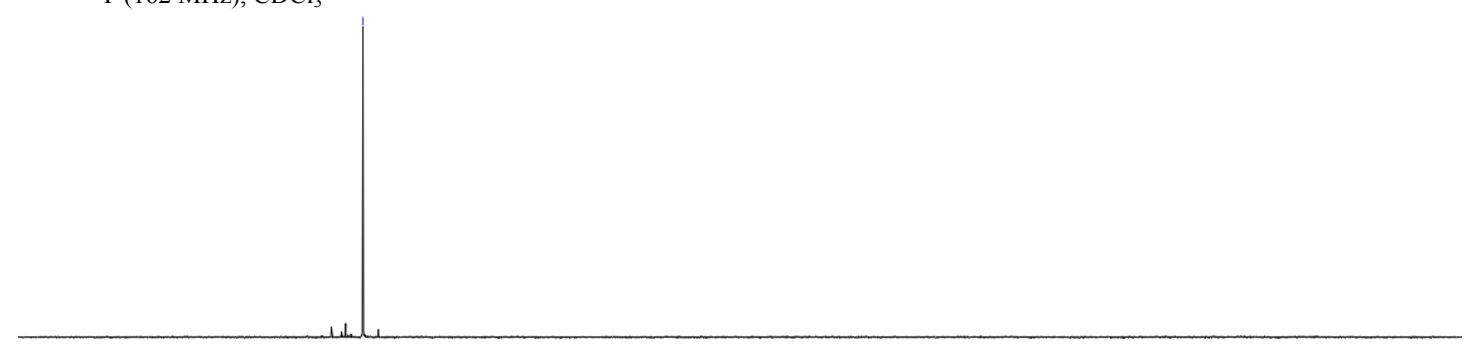

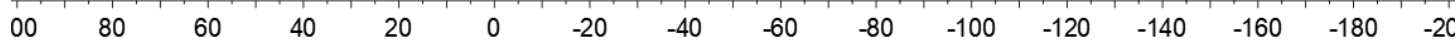




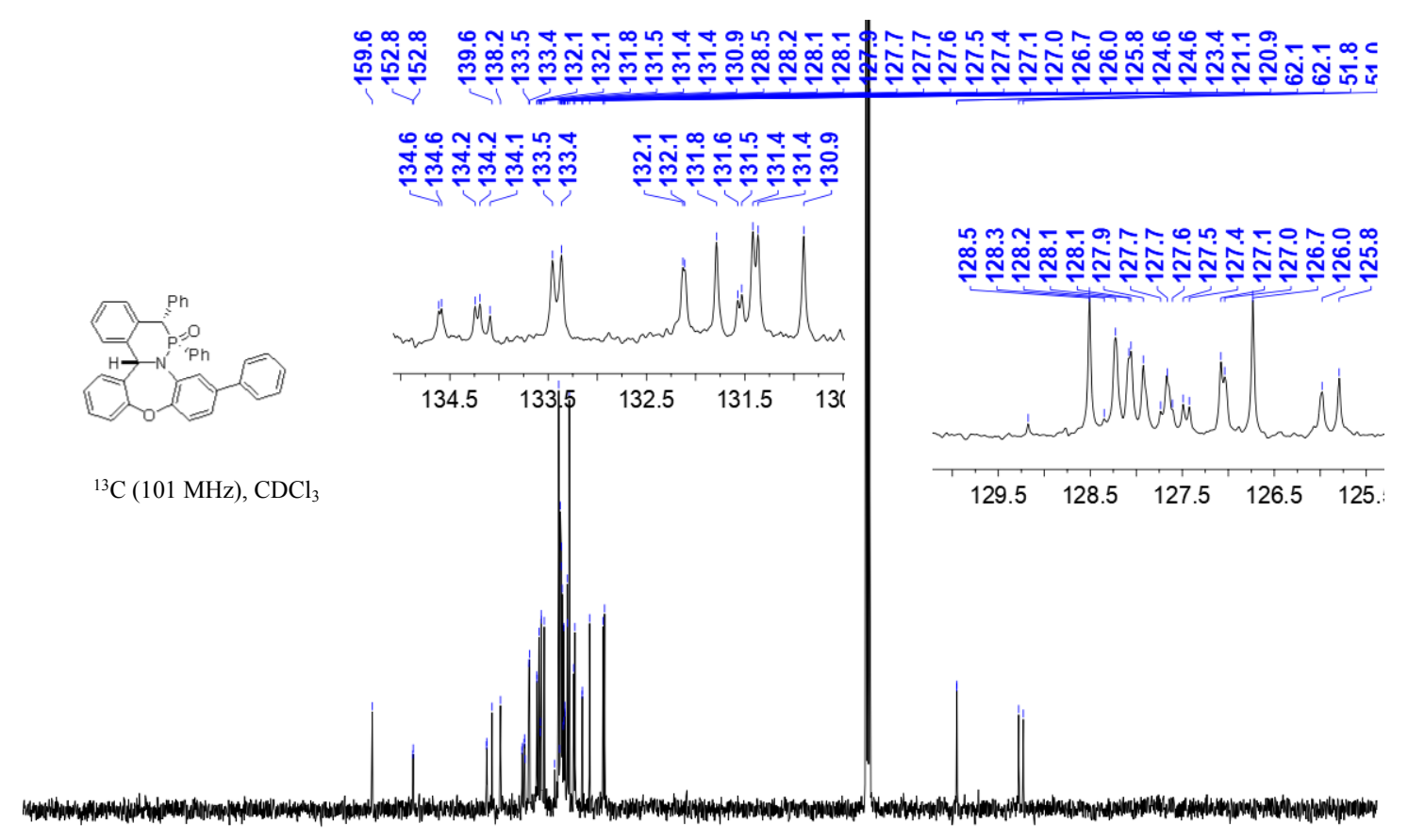

$\begin{array}{llllllllllllllllllllll}210 & 200 & 190 & 180 & 170 & 160 & 150 & 140 & 130 & 120 & 110 & 100 & 90 & 80 & 70 & 60 & 50 & 40 & 30 & 20 & 10 & 0\end{array}$

${ }^{1} \mathrm{H}(400 \mathrm{MHz}),{ }^{31} \mathrm{P}(162 \mathrm{MHz})$ and ${ }^{13} \mathrm{C}(101 \mathrm{MHz})$ spectra of compound 4 